This item was submitted to Loughborough's Research Repository by the author.

Items in Figshare are protected by copyright, with all rights reserved, unless otherwise indicated.

\title{
Synthetic aperture sonar
}

PLEASE CITE THE PUBLISHED VERSION

PUBLISHER

(c) Avtar Singh Gida

LICENCE

CC BY-NC-ND 4.0

REPOSITORY RECORD

Gida, Avtar S.. 2019. "Synthetic Aperture Sonar”. figshare. https://hdl.handle.net/2134/11120. 


\section{Loughborough University}

This item was submitted to Loughborough University as a PhD thesis by the author and is made available in the Institutional Repository

(https://dspace.lboro.ac.uk/) under the following Creative Commons Licence conditions.

\section{cc) creative}

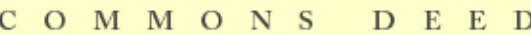

Attribution-NonCommercial-NoDerivs 2.5

You are free:

- to copy, distribute, display, and perform the work

Under the following conditions:

Attribution. You must attribute the work in the manner specified by the author or licensor.

Noncommercial. You may not use this work for commercial purposes.

No Derivative Works. You may not alter, transform, or build upon this work.

- For any reuse or distribution, you must make clear to others the license terms of this work.

- Any of these conditions can be waived if you get permission from the copyright holder.

Your fair use and other rights are in no way affected by the above.

This is a human-readable summary of the Leqal Code (the full license).

Disclaimer 민

For the full text of this licence, please go to: http://creativecommons.org/licenses/by-nc-nd/2.5/ 


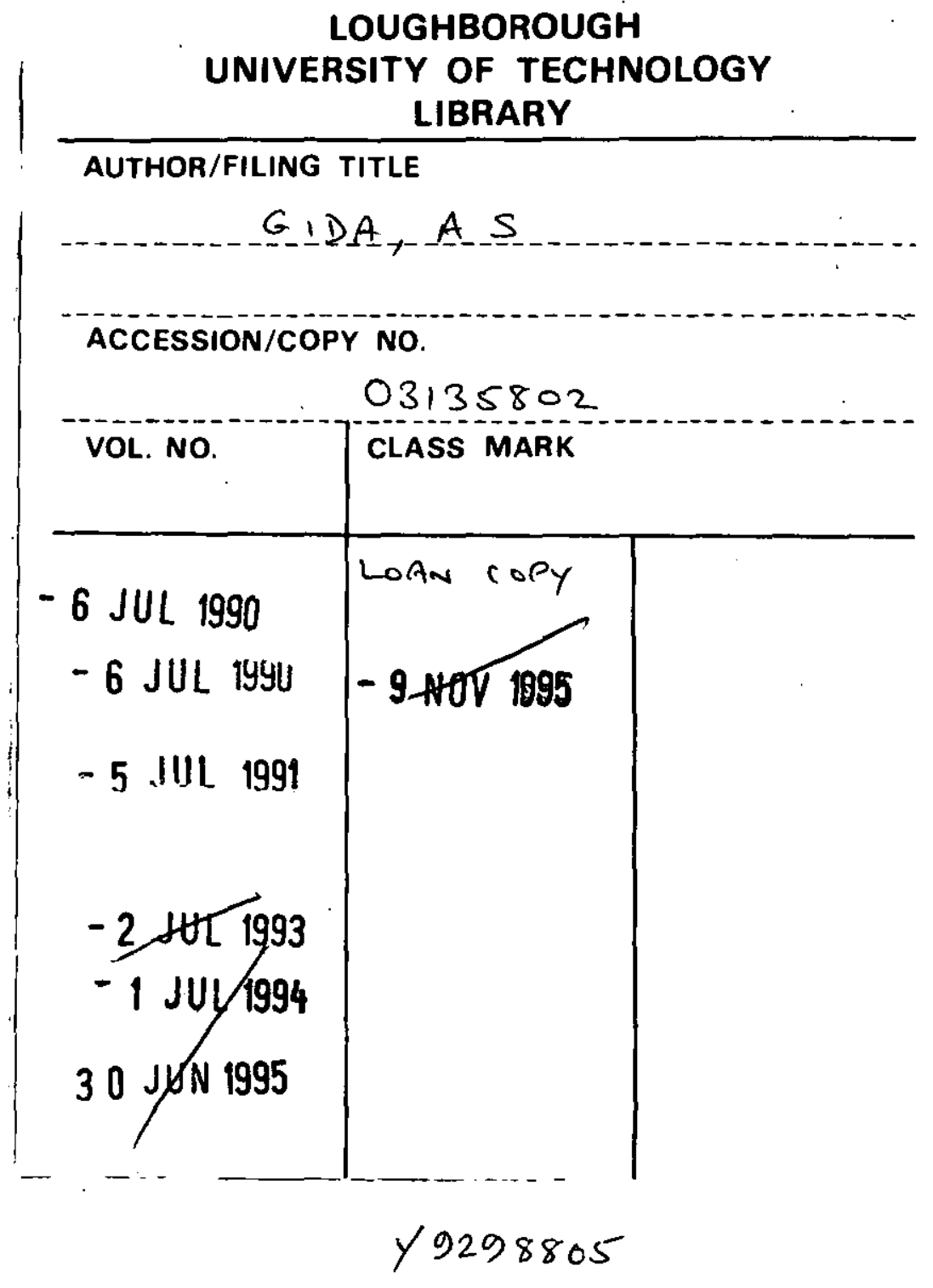

$003 \quad 1358 \quad 02$

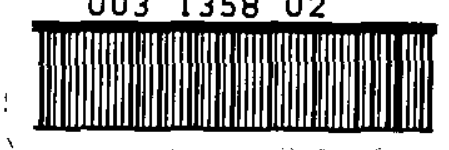




\section{SYNTHETIC APERTURE SONAR}

by

AVTAR SINGH GIDA, B.Sc.

A Doctoral Thesis Submitted in partial fulfilment of the requirement for the award of Doctor of Philosophy of the University of Technology, Loughborough, England.

April 1988.

Supervisor: Professor J.W.R. Griffiths.

Department of Electronic:and Electrical Engineering, Loughborough.

(C) by Avtar Singh Gida, 1988. 


\section{ACKNOWLEDGEMENTS}

I would like to thank my supervisor, Professor J.WR. Griffiths, for his encouragement and constructive criticism throughout the course of this research and for providing research facilities and financial support.

$I$ would also like to thank the Science and Research Engineering Council for providing the financial assistance through a research studentship and Dr. J. Cook for providing financial assistance through a research assistantship.

I am also indebted to my colleges in the department, A.D. Goodson, $J$. Goodge and Dr. V. Vadher for reading the final drafts of this thesis.

Finally I would like to thank my wife, Vali, without whose moral support and encouragement I would not have been able to complete this work.

To Vali I dedicate this thesis. 


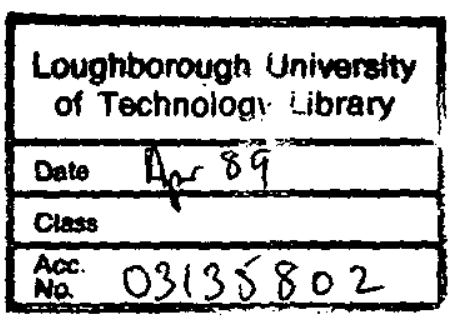




\section{SYNOPSIS}

Synthetic aperture techniques have been applied very successfully for many years in astronomy and radar to obtain high resolution images, an outstanding example in recent years being the use in remote sensing satellite systems. In underwater acoustics, because of the inherent problems caused by random fluctuations in the signal path, the slow velocity of the acoustic wave and the unknown movements of the transducer as it traverses the aperture, the application of the synthetic aperture technique has mainly been limited to the very useful but rather inferior non-coherent technique known as side-scan sonar. However the rapid advances that are being made in micro-chip technology and fast digital signal processing, and the development in image processing algorithms has created renewed interest in the possible application of the synthetic aperture technique to underwater acoustics. This thesis describes such a study.

After giving a brief overview of the subject the thesis describes work done on computer simulation to predict the performance with and without impairment and with the use of image processing. It gives details of the design and construction of a synthetic aperture sonar system which includes a method to reduce the degradation in the image caused by deviations of the transducer motion from the intended path in the aperture,

Initially experimental work was carried out in a large tank in the department but then a system with a much larger aperture was built and tested at a nearby reservoir. The experimental results are reported. 


\section{CONTENTS}

GLOSSARY

CHAPTER 1: INTRODUCTION

page

1. 1 Introduction 1

1.2 Objectives 3

1. 3 Summary: Chapter by Chapter 4

CHAPTER 2: SYNTHETIC APERTURE: A BRIEF OVERVIEW

2.1 Introduction 6

2.2 Early Developments in Synthetic Aperture Systems 7

2. 3 Definition of Resolution 13

2. 4 Transducer Radiation Pattern 13

2.4.1 Radiation Pattern of a Continuous Aperture 14

2.4.2 Radiation Pattern Transducer Arrays 16

2.5 Resolution Achievable using Linear Arrays 18

2. 6 Resolution Achlevable using Synthetic Arrays 21

2.6.1 Unfocussed Synthetic Arrays 25

2.6.2 Focussed Synthetic Arrays 27

2.7 Grating Lobes 31

2.8 The Sonar Equation 32

2.8.1 The Definition of Source Level 34

2.8.2 The Definition of Target Strength 34

2.8.3 The Definition of Transmission Loss 35

2.8.3.1 Transmission Loss due to Spherical

Spreading 36 
2.8.3.2 Transmission Loss dwe to Cylindrical Spreading 36

2.8.3.3 Transmission Loss due to Absorption 38

2.9 Restrictions in the Use of Synthetic Aperture Sonar Systems

2.9.1 Limitations due to the Low Propagation Velocity of Sound in Water 40

2.9.2 The Effects of Media Turbulence

2. 9.3 Transducer Motion Errors

2.9.4 Image Degradation due to Target Motion

2. 10 Radar Apertures and Sonar Apertures Compared

\section{CHAPTER 3: SIGNAL PROCESSING TECHNIQUES}

3. 1 Introduction

3. 2 Optical Signal Processing

3. 3

Digital Signal Processing

51

3. 3.1 Signal Processing in the Frequency Domain

3.3. 2 Signal Processing in the Time Domain

3. 3.3 Baseband Representation of Band Limited Signals

\section{CHAPTER 4: SIMULATION}

4. 1 Introduction

64

4.2 Simulation for a Single Target

64

4. 3

Simulation for Two Targets

65

4. 4

Simulation of Motion Errors

68

4.4.1 Errors due to Random Transducer Motion 70

4.4.2 Errors due to Blased Transducer Motion 70

4. 5 Transducer Error Motion Correction 
5. 1 Introduction

5. 2 Cont inuous Image Model

5.3 Digital Image representation 102

5.4 The Two Dimensional Filter 103

5.5 Image Enhancement 105

5.5.1 Smoothing 105

5.5.1.1 Simple Neighbourhood Average 105

5.5.1.2 Smoothing with a Threshold 106

5.5.1.3 Median Smoothing 109

5.5.2 Edge Sharpening . 111

5.5.3 Contrast Enhancement 111

5.5.3.1 Linear Scaling Transformation 112

5.5.3.2 Piecewise Linear Transformation 112

5.5.3. 3 Histogram Modification 114

5.6 Image Restoration 115

5.6.1 Deconvolution 115

5.6.2 Subtractive Convolution $\quad 116$

CHAPTER 6: SYSTEM HARDWARE AND SOFTWARE

6.1 Introduction 121

6.2 System Hardware 121

6.2.1 The BBC Microcomputer \& 32016 Comprocessor 121

6.2.2 The I and $Q$ Baseband Receiver Board 123

6.2.3 The Memory Buffer Board . 128

6.2. 4 Range Select Facility 131

6.2.5 The Transmit Pulse and Reference Generator Board 132

6.2.6 The Stepper Motors 134

6. 3 System Software and Operation 136

6. 3.1 The Data Collection Procedure 136

6.3.2 Image Reconstruction using the Co-Processor 138

6. 4 The Development of a $40 \mathrm{kHz}$ System 142 
7.2 Experiments in the Department Tank

7.2.1 Images Obtained using the $150 \mathrm{kHz}$ System

7.2.2 Images Obtained using the $40 \mathrm{kHz}$ System

7.3 Experiments at the Reservoir

7.3.1 Images without Transducer Motion Error Correction

7.3.2 Images with Transducer Motion Errors Correction and Image Processing

CHAPTER 8: CONCLUSION AND SUGGESTIONS FOR FUTURE WORK

\section{REFERENCES}

\section{APPENDICES}

Appendix 1: The British Telecom Framestore

Appendix 2: The Velocity of Sound in the Sea

Appendix 3: The Stepper Motors

Appendix 4: The User Port Connections

Appendix 5: The Receiver Board and Memory Buffer Ribbon Cable

Appendix 6: Full Circuit Diagram of the Transmit Pulse and Reference Generator Board

Appendix 7: Full Circult Diagram of the Memory Buffer Board

Appendix 8: Full Circuit Diagram of the Baseband Receiver Board 


\section{GLOSSARY}

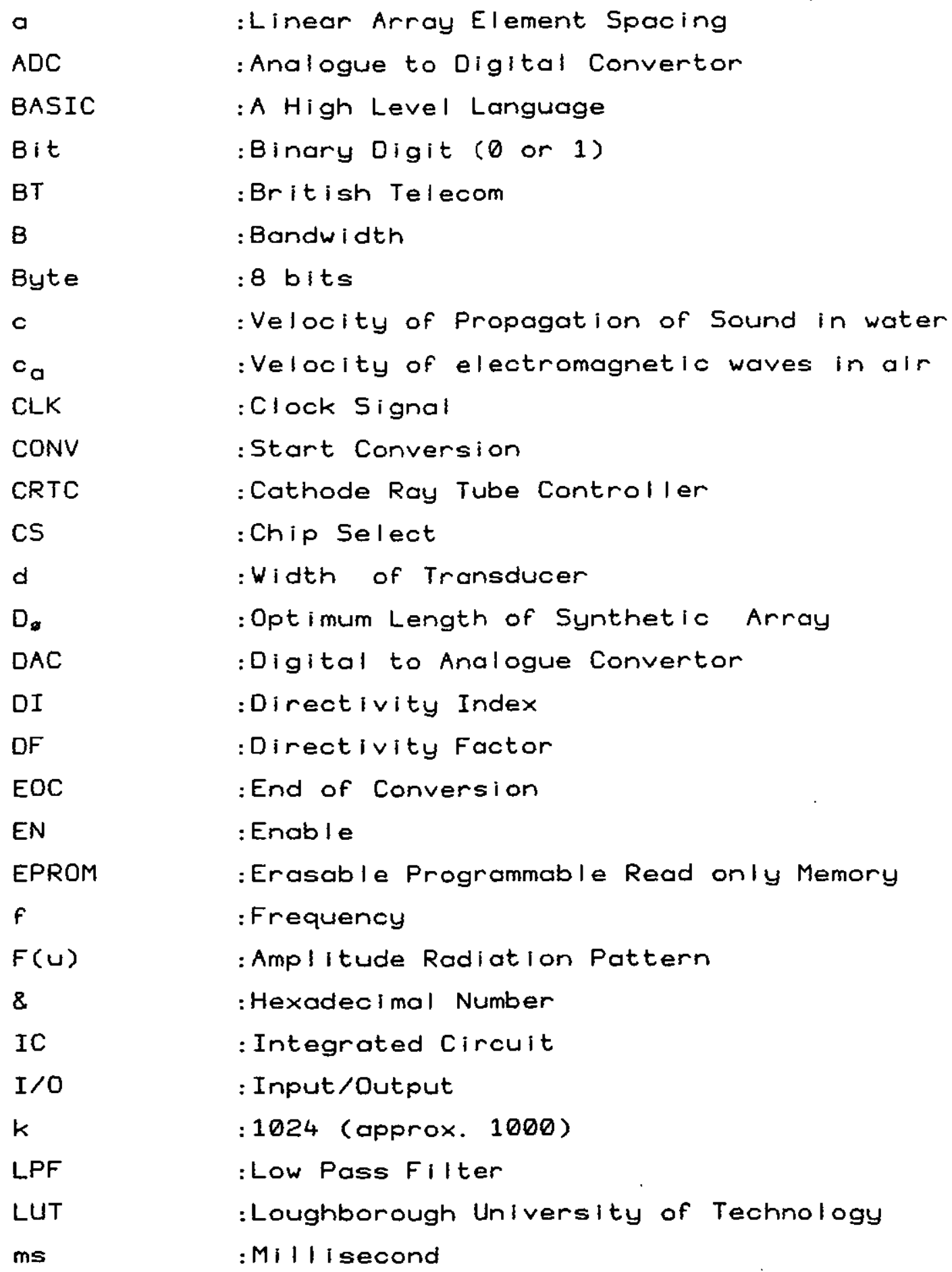




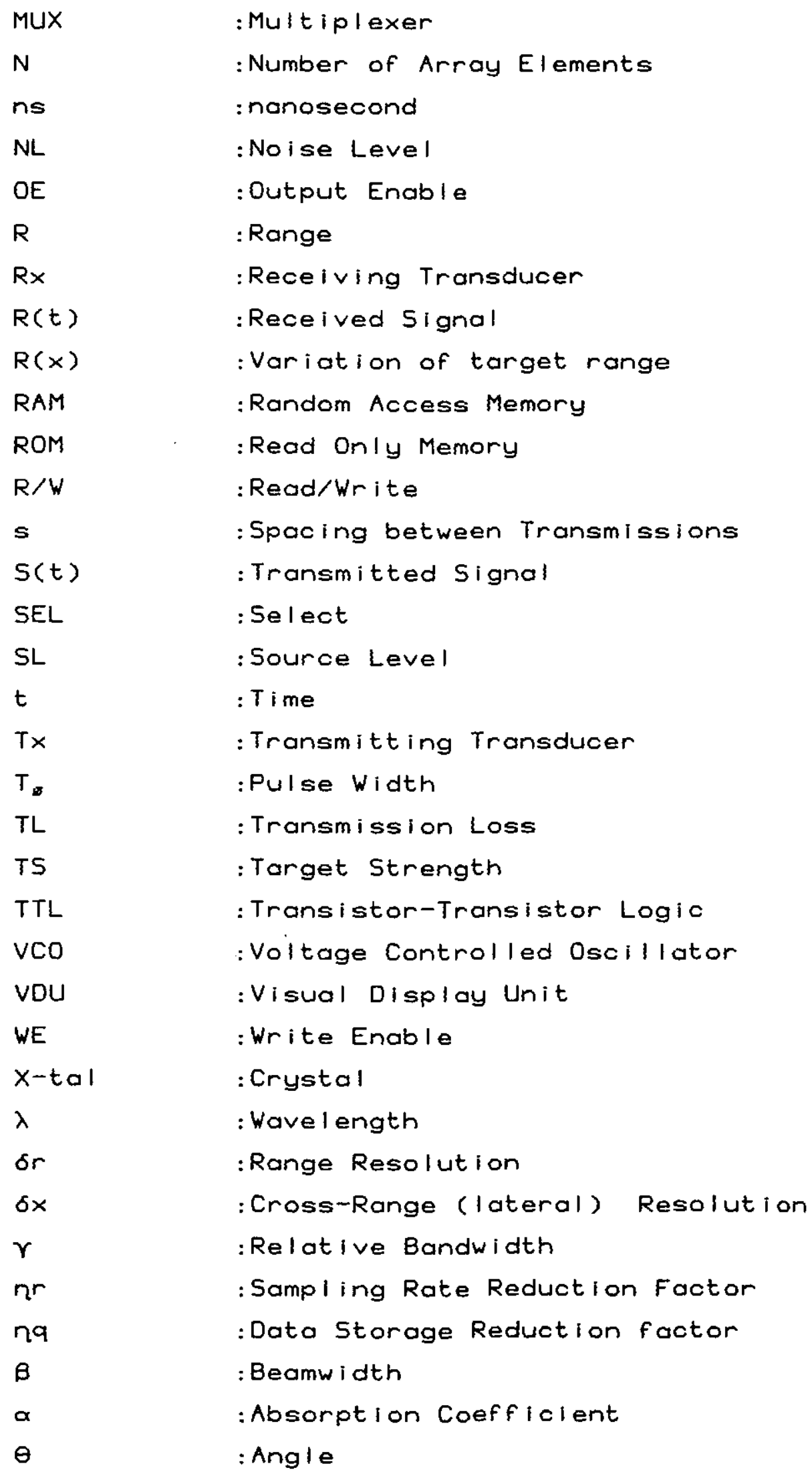


$\Phi$

$\omega$

$\tau$

○

6502

:Phase Shift

:Carrier Signal

:Time Delay

: Convolution

: 8 bit Microprocessor used in the BBC

Microcomputer

32016

: 32 bit Microprocessor 


\section{INTRODUCTION}

\section{1 Introduct ion}

Sonar, an acronym for Sound, NAvigation and Ranging. is the generic term used for the various systems which detect, locate and classify underwater targets by acoustic means. Underwater sound sources have been in use since the turn of the century, but it was not unt il the advent of the thermionic valve that sonar systems weré developed. Since that time, the initial concepts have been developed extensively and currently very many systems are in use.

The resolution of an imaging system is the minimum separation between two targets or sources or scattering centres on a body at which they can be separately dist inguished.

Good range resolution can be achieved by the use of short transmitted pulses, but this often limits the energy transmitted and the maximum range of operation if the transmitter is peak-power limited. Pulse compression techniques allows radar and sonar systems to utilize a long pulse to achieve large radiated energy, but simultaneously to obtain the range resolution of a short pulse. This is accomplished by employing frequency or phase modulation to widen the signal bandwidth and using a matched filter in the receiver that compresses the long pulse to duration $1 / B$. where $B$ is the modulated pulse spectral bandwidth. 
The achievable. azimuth (lateral) resolution is proportional to the signal wavelength and inversely proportional to the array size. The use of high frequency signals is limited by the transmission loss due to absorption in underwater applications. The conventional technique for achieving good azimuthal resolution has been that of radiating a narrow beam by the use of a long array. but there are physical limitations to the maximum size. In addition to the practical difficulties in construction, installation and operation of a large array there is the Iimitation imposed by the near-field of the array.

A synthetic aperture system achieves high resolution in the cross-range direction by taking advantage of the motion of the vehicle carrying the system to synthesize the effect of a large aperture. The absolute lateral resolution achievable using conventional linear arrays is proportional to array length measured in wavelengths and range. The resolution achievable using the synthetic aperture technique, is independent of range and frequency and proportional only to the physical size of the tronsducer. giving the possibility for generating very long apertures and thus obtaining very high lateral resolution.

In radar, the synthetic aperture has been used successfully for many years in aircraft and satellite high resolution terrain mapping systems. Small moving aerials have also been used in radio astronomy to synthesize large apertures and obtain narrow pencil beams.

The application of. the synthetic aperture technique to underwater systems has been limited due to the problems incurred in maintaining coherency of the received signals along the aperture. Phase errors due to turbulence in the 
water or due to unknown movement of the transducer can seriously degrade the image. Target motion during the time taken to traverse the length of the synthetic aperture leads to a defocussed image and a shift in the position of the reconstructed target. Another restriction. Which is relevant to all sonar systems, is that the low propagation velocity of sound in the sea imposes a limit on the maximum pulse repetition frequency. Normally to avoid ambiguity, the echo from the farthest range of interest must be received before the next pulse can be transmitted. and this results in a low mapping rate.

\subsection{Objectives}

The main objective of this research project was to conduct a study to examine the use of the synthetic aperture technique to underwater applications and to consider the various design parameters that affects its real-time implementation.

The project commenced with the construction of a simple synthetic aperture sonar system in a well controlled environment in the department tank to obtain high resolution underwater images. An operating frequency of $150 \mathrm{kHz}$ is used to keep the length of the generated aperture small for operation in the tank.

A move to a more realistic and less controlled situation took place by constructing another system operating at $40 \mathrm{kHz}$ for deployment at a local reservoir. A lower operating frequency was used to reduce the effects of transducer position uncertainty and obtain the advantages of a lower absorption coefflcient. 
The final part of the project included work to simulate the effects of unknown transducer motion and develop a system to reduce the deterioration in the images due to these effects.

\section{3 Summary: Chapter by Chapter}

The historical development of the synthetic aperture and its early uses in radar and sonar is discussed in Chapter 2. Radiation patterns and beamwidths are discussed for continuous apertures and I inear arrays with the following section considering resolution achievable using Iinear arrays and the two types of synthetic arrays. unfocussed and focussed. The basic parameters influencing the sonar equation are considered and restrictions caused by the inhomogenities and turbulence in the water are reported and the effects of target motion and transducer motion errors discussed.

Chapter 3 reviews the optical and digital methods used in collecting and processing the data to obtain a two dimensional image of the area of interest. The frequency and time domain digital processing techniques are examined and an analysis of the use of baseband quadrature components of the received signal, to reduce the digital data sampling rate and the digital storage requirements of the system is olso carried out.

System simulation is carried out for a single target and a pair of targets in Chapter 4. The expected data values and the expected images are presented, and the effects of transducer motion on the reconstructed image are considered by simulating the system with random and biased transducer movement. The amplitude of the random motion and the amplitude and frequency of the biased motion is 
varied and the influence on the reconstructed image presented. Finally a technique to reduce the effects of unknown transducer motion is simulated and the results il lustrated.

A brief discussion of the image processing required to enhance the reconstructed images is carried out in chapter $\underline{5}$ and the advantages of using smoothing and subtractive deconvolution techniques are briefly examined.

Chapter 6 contains the details of the hardware and software design of the synthetic aperture system. A board by board description of the complete system is provided and the procedures for collecting the data and reconstructing the final image are presented. The complete circuit diagrams of the boards are included in the appendices.

The results obtained using the $150 \mathrm{kHz}$ system and the $40 \mathrm{KHz}$ system in the tank laboratory are presented in Chapter 7. A modified version of the $40 \mathrm{kHz}$ system is used at a local reservoir and the images obtained using the transducer motion error correction technique discussed in chapter 4 and image processing techniques are compared with the results obtained without transducer motion error correction and without image processing. 


\section{SYNTHETIC APERTURE:} A BRIEF OVERVIEW

\section{1 Introduction}

The principles and processing techniques of synthetic aperture have been applied to high-resolution terrain mapping radar. radio astronomy. ultrasonic imaging and sonar for many years. The main advantage of the synthetic aperture technique is that fine resolution can be achieved using a small physical transducer or antenna. The fine resolution is obtained by coherently summing the echoes of successive transmissions as the antenna or transducer moves to take up adjacent element positions in a synthetic aperture.

Section 2.2 discusses the early development in synthetic aperture systems, in the field of radio astronomy, radar and sonar and the next section introduces the radiation patterns of transducers and of linear arrays. The resolution achievable using conventional arrays and synthetic arrays is compared. Section 2.7 discusses the problems of grating lobes and the following section briefly considers the sonar parameters and the sonar equation. Section 2.9 describes the problems of using any type of sonar system in the sea and the parameters that have restricted the use of synthetic arrays and the methods developed to overcome them. 


\subsection{Early Developments in Synthet ic Aperture Systems}

In the field of radio astronomy, aerials of large aperture, giving norrow beams, were difficult to realize especially at low frequencies. Blythe [72,1] used the synthetic aperture technique to synthesize a large aperture by using a small moving derial to produce a narrow pencil beam at the Cavendish Laboratory in Cambridge. Biythe [1] credited the late Prof. M. Ryle, for the original suggestion of aperture synthesis.

The use of a synthetic aperture, in the field of radar. is credited to Carl Wiley. whilst working for the Goodyear Aircraft Corporation in 1951. He observed that o radar beam orientated obliquely to the radar vehicle will receive signals having frequencies offset from the radar carrier frequency due to the Doppler effect [2].

Consider the configuration shown in Fig. 2.1. where an aircraft. With a velocity of $\nu$ is carrying a coherent

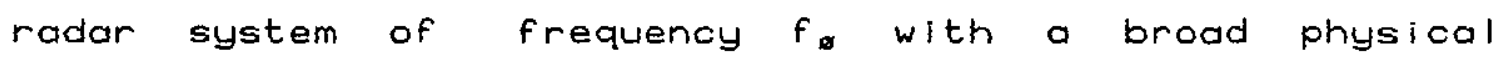
beamwidth. $\theta_{1}$ is the angle between $\nu$ and the line from the radar platform and a reflecting point, $T_{1}$.

The Doppler shift, $f_{1}$. in the frequency of the reflected signal is given by

$$
f_{1}=\frac{(2 \nu) f_{0}}{c_{0}} \cos \theta_{1}
$$

where $c_{a}$ is the velocity of propagation of electromagnetic waves in air.

If a second reflecting point. $T_{2}$, is located at a slightly different angle, $\theta_{2}$. then its reflected signal 


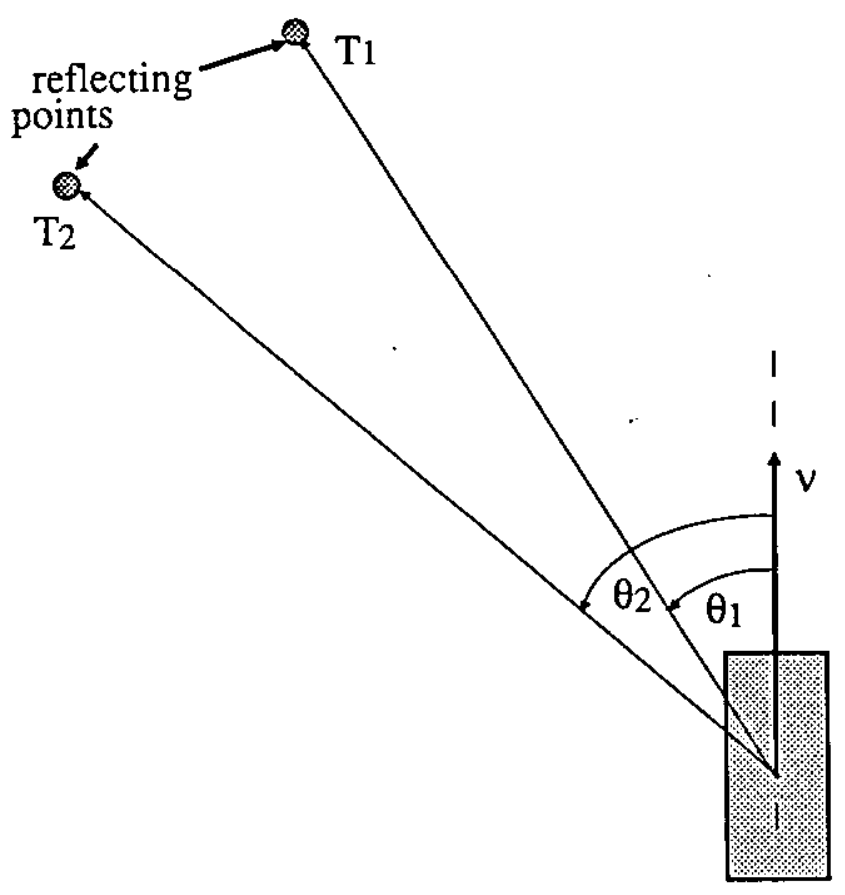

radar platform

Fig. 2.1 Synthetic_Aperture Radar Geometry.

will have a Doppler shift $f_{2}$. The two Doppler frequencies $f_{1}$ and $f_{2}$ will differ by $\Delta f$. where



[2.2]

If both targets are stationary, the measurement of $f_{1}$ and $f_{2}$ leods to the two angles $\theta_{1}$ and $\theta_{2}$. In the event that a target is moving. an additional Doppler shift is added as a result of the radial component of its motion. and the angular position of the target connot be determined accurately, unless the additional velocity of motion is known.

In the case where $\sin \theta$ is unity, i.e. looking in o direction normal to the flight path. the Doppler frequency 
approaches zero. but the rate of change of the Doppler frequency with $\theta$ is a maximum.

Concurrently to Wileys work, experimental observations were underway at the University of IIlinois, on the nature of radar reflections of ground targets, as observed by an airborne non-coherent $X$-band radar system. The aim of the experiments was to study the non-coherent Doppler signals resulting from both moving and non-moving targets. Distinctive frequency spectra containing sharp lines were observed from certain terrain samples. It was concluded that the sharp frequencies must be due to a small number of strongly reflecting fixed targets.

Sherwin [2] later applied this principle to a pulsed, coherent radar system with a relatively broad beamwidth. He synthesized an extremely long antenna by using the forward motion of the aircraft to carry a side-looking radar antenna to positions which could be treated as though they were the individual antenna elements of a linear array. Discussions between Dr. C. Sherwin of the University of Illinols, W. Hausz of the General Electric Company, J. Koehler of the Philco Corporation and Dr. L. Cutrona of the University of Michegan lead to the development of the first airborne synthetic aperture radar system mounted in a USAF-C46 aircraft in 1957. This research lead to the development of a new type of combat survelllance radar system designated the AN/UPD-1 [3] and unveiled in April 1960 by the US army.

The first application of an incoherent synthetic aperture technique to underwater systems was in the form of side scan sonar, where a transducer is towed in a stralght Iine and a two dimensional image is formed from the incoherent proccessing of the recelved echoes from 
successive transmissions. The technical base for side scan sonar was developed during the antisubmarine war effort of World War II and the subsequent defence research [69]. But it was not unt il 1958 that the first open publication appeared on sidescan sonar $[70,71]$.

Side scan sonar systems generate a two dimensional image from the strength of acoustic backscattering from the sea floor. The strength of the backscattering from an object is not an absolute value, but that observed from the particular aspect of the transducer position. A given object could produce a different image intensity if observed from another position, or indeed at a different frequency. Scanning takes place in two directions, namely along the track of the transducer and perpendicular to it.

The perpendicular or crosstrack scanning is achieved by the passage of sound waves through the water, the reception of echoes from successively greater ranges occurring at later and later times from the instant of transmission. On sea floor which is level and fairly flat. image range increases monotonically though not I inearly with actual range. Thus a marking stylus moving uniformly across the image medium marks all the echoes in the right order, but with a distortion. This distortion is severe for objects under the track of the transducer, but dies away to become negligible when the range is several times the height of the transducer above the sea floor. This "slant range distortion" was endured in the early years of side scan sonar because of the difficulty of finding an effective cure by analogue methods. With the advance of digital techniques it became relatively simple to perform the necessary corrections.

Along track scanning is achieved by physical movement 
of the transducer. In this direction, scanning is not continuous and the sea floor is sampled by the sequence of discrete pulse transmissions as the transducer is moved along a known path.

The typical side scan sonar system consists of a towed sensor called a "fish". a tow cable and a graphic recorder. The fish produces short, high intensity, ultrasonic pulses which are projected in a narrow fan-shaped beam out perpendicular to the track of the towfish. Figure 2.2 shows a system using two separate transducers. one for each side [81]. The graphic recorder also has two channels to enable port and starboard data to be simultaneously displayed.

The operating frequencies of side scan sonar systems vary from about $5 \mathrm{kHz}$ for large range operation to 500 $\mathrm{kHz}$ for higher resolution and smaller ranges. Recorders also have the capability to enable the recording of the data on to magnetic tape and other media. This allows the making of as many copies of the record as needed and post processing using computer techniques. CRT displays are also used to display and input additional positioning information.

The range resolution of a side scan sonar is related to the width of the transmitted pulse and is equal to cT, $/ 2$, where $T_{g}$ is the pulse width. The along track resolution. on the other hand, is either the width of the horizontal beam on the sea floor or the distance travelled by the transducer in between transmissions, whichever is the worse. At close range the latter is worse, but as the range increases the former deteriorates as the horizontal beam spreads over a larger and larger lateral distance. This leads to the impression that objects at far ranges 


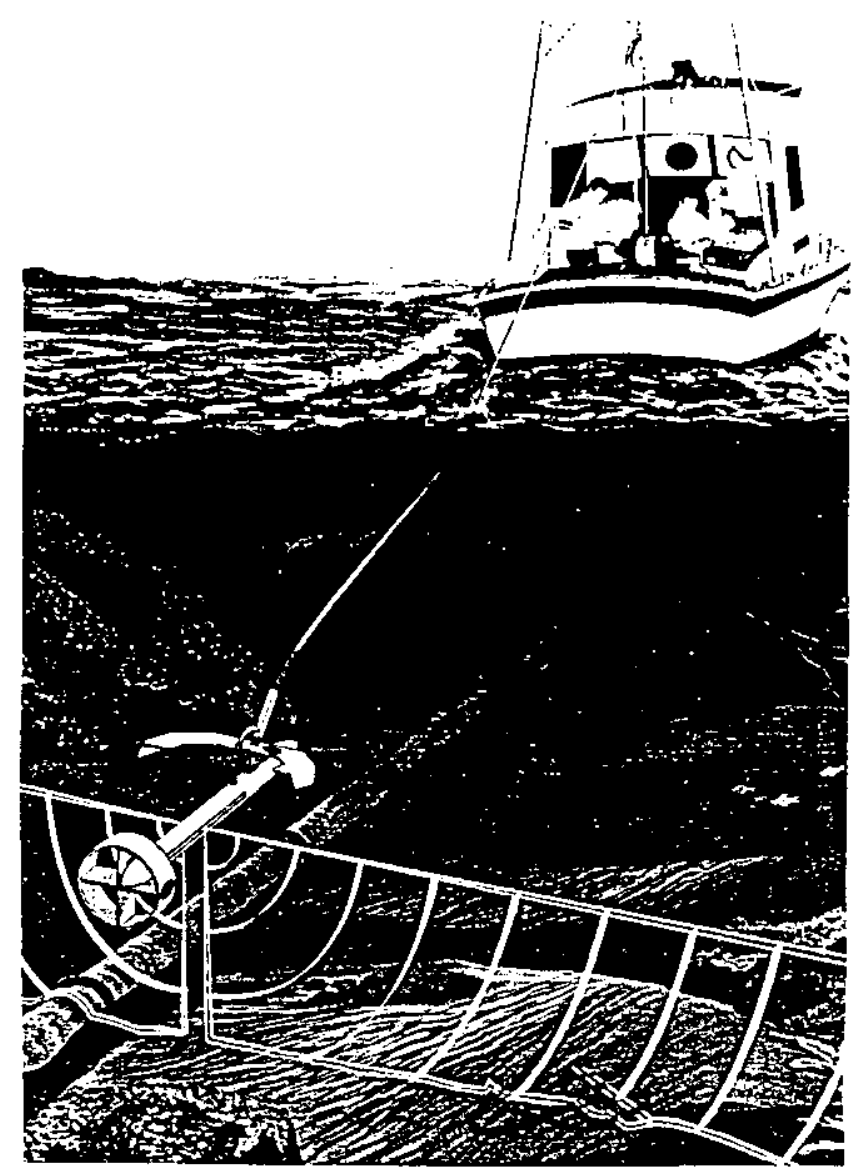

Fig. 2.2 A Side-scan Sonar System Using Two Transducers.

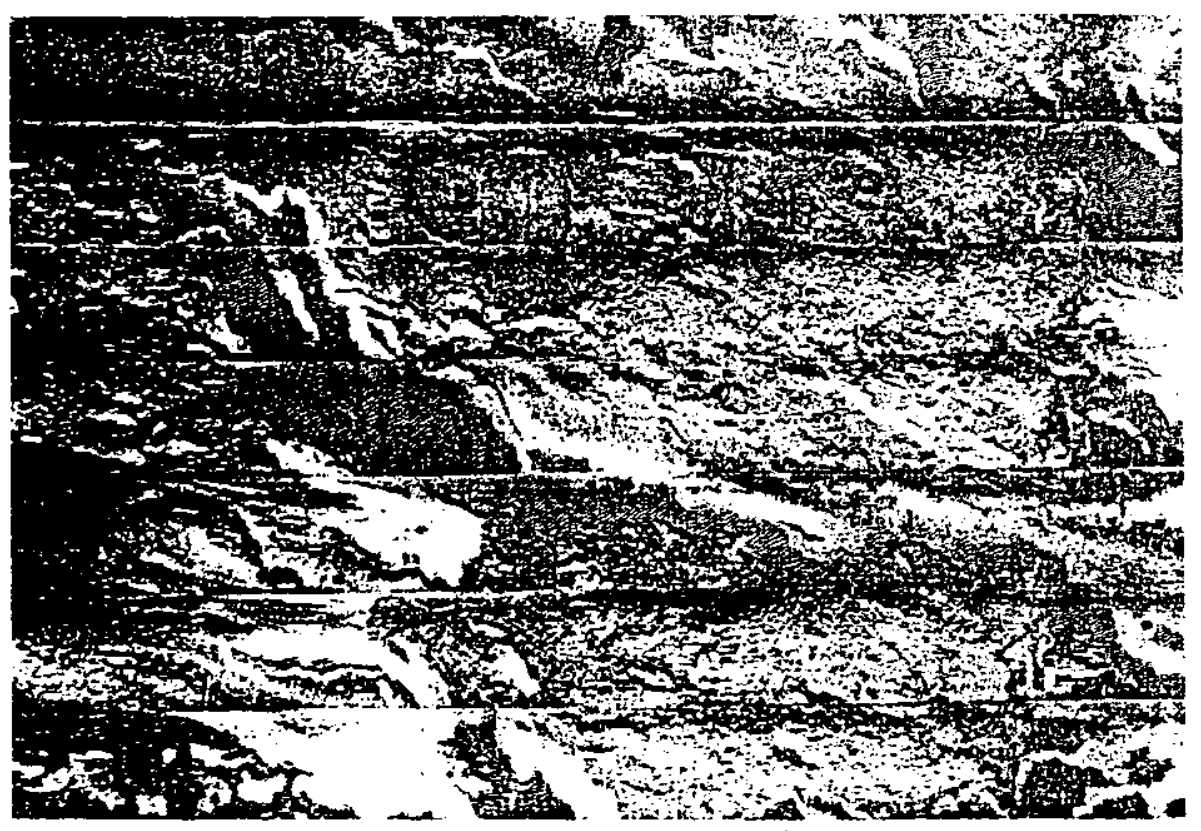

Fig. 2.3 A side-scan sonar Mosaic of the sea floor. (Af ter Kozak [81]) 
have a I inear structure parallel to the transducer track. This effect is undesirable and the transducer is designed to have the narrowest possible horizontal beamwidth. The vertical beamwidth, on the other hand, must be sufficient to cover the total range swathe to be imaged.

Slant range and speed correction techniques using digital techniques allow the generation of a completely distortion-free record, where the aspect ratio is a true one:one. This allows the making of mosaics as illustrated in Fig. 2.3 [81] showing a large portion of the sea floor.

\section{3 Definition of Resolution}

Resolution as discussed in this thesis is a measure of the beamwidth of the main lobe of the effective receiver pattern and not a measure of the ability to distinguish objects a certain distance apart, because the latter description is a function of the scattering properties and orientation of the isonified object.

The next section will discuss the radiation patterns and beamwidths obtained using continuous aperture and linear arrays.

\subsection{Transducer Radiation Patterns}

A transducer which is being used as a transmitter does not radiate uniformly in all directions. or conversely, a transducer being used as a receiver does not detect energy uniformly from all directions. This directional selectivity of a transducer is characterised in terms of its radiation pattern. which is a plot of the relative strength of the radiated field as a function of angular position. 


\section{$\mathrm{A}^{+}$ \\ 5}

CHAPTER TWO

The most common forms of illustrating the radiation patterns are as two dimensional patterns with either polar or cartesian coordinates. The cartesian representation will be used in this thesis.

The radiation pattern may be used to obtain the beamwidth of the main beam. This is normally specified as the angular difference between the points on the radiation pattern where the power has fallen to one half of the peak value or $-3 \mathrm{~dB}$ on the decibel scale. We shall first consider the radiation pattern of a line aperture and of a circular aperture and then the radiation pattern of an array of transducers and discuss the effects of size. shape and distance between the array elements.

\subsubsection{Radiation Pattern of Continuous Apertures}

The far field amplitude pattern $F(u)$ of a line source with constant excitation can be expressed as

$$
\begin{aligned}
& \quad F(u)=\frac{\sin (\pi u)}{\pi u} \\
& \text { where } u=\frac{d \cdot \sin (\theta)}{\lambda} \\
& \theta=\text { angle. with broadside }=0^{\circ} \\
& \text { and } \quad d=\text { length of transducer. }
\end{aligned}
$$

The $\sin (x) / x$ distribution is very important and is plotted in Fig. 2.4. The level of the first sidelobe is $13.2 \mathrm{~dB}$ below the peak level of the main beam and the field has a null at $d . \sin (\theta) / \lambda .= \pm 1, \pm 2, \pm \ldots$ The width of the main beam is another important factor and in this case the half power or $-3 \mathrm{~dB}$ beamwidth is $0.88 \lambda / d$ (radians). so the beamwidth is inversely proportional to the aperture 
size. The sidelobe level can be reduced considerably by a tapered aperture distribution where the field is greatest at the centre and reduces to a low level at the edges. The cosine distribution. for example, reduces the level of the first sidelobe to $-23 \mathrm{~dB}$ but broadens the main beamwidth to 1. $2 \lambda / d[90]$.

The radiation pattern for a circular aperture with constant excitation is

$$
F(u)=\frac{2 \cdot J_{1}(\pi u)}{\pi u}
$$

where $J_{1}$ is the first order Bessel function.

The sidelobe level in this case is $-17.6 \mathrm{~dB}$ but the beamwidth is slightly broader ot $1.2 \lambda / d$ with $d$ specifying the diameter of the circular aperture.

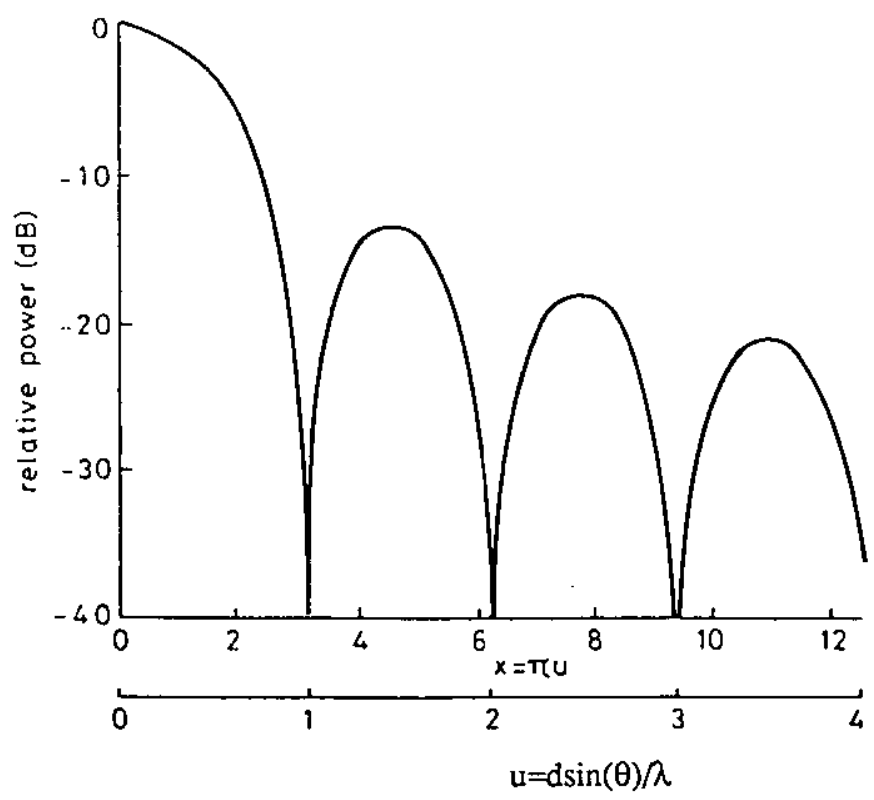

Fig.2.4 $\operatorname{Sin}(\mathrm{x}) / \mathrm{x}$. Amplitude Badiation Pattern. 


\subsubsection{Radiation Pattern of Transducer Arrays}

Arrays are made up of a matrix of discrete sources. which radiate individually but the pattern of the array is also determined by the relative amplitude and phasing of the excitation fields on each source and the geometric spacing of the sources. The total radiation pattern is the multiplication of the pattern due to an individual element. the element factor. and the pattern due to an array of omnidirectional sources, the array factor.

Consider a linear array of $N$ elements equally spaced a distance a apart as illustrated in Fig. 2.5. The outputs of the elements are summed to provide the output signal $E$. Assuming element 1 is taken as the reference signal with zero phase. The difference in the phase of the signal at adjacent elements is $\Phi$ where

$$
\Phi=\frac{2 \pi \sin (\theta)}{\lambda}
$$

and where $\theta$ is the direction of the incoming signal.

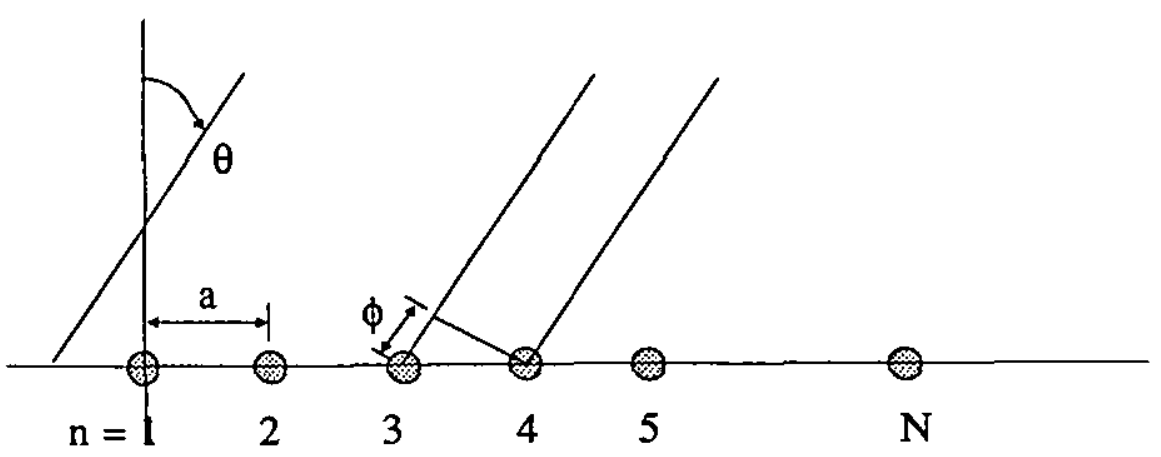

Fig. 2.5 Linear Array Geometry. 
If the amplitudes and phases of the stgnals are weighted unfformly, the output signal can be written as

$$
E=\sin (\omega t)+\sin (\omega t+\phi)+\sin (\omega t+2 \phi)+\ldots[2.7]
$$

therefore

$$
E=\sin [\omega t+(N-1) \Phi / 2] \frac{\sin (N \Phi / 2)}{\sin (\Phi / 2)}[2.8]
$$

The recelved amplitude therefore 18

$$
\frac{\sin (N \pi(a / \lambda) \sin (\theta))}{\sin (\pi(a / \lambda) \sin (\theta))}
$$

The recelver pattern has nulls when

$$
N \pi(a / \lambda) \sin (\theta)= \pm \pi, \pm 2 \pi \ldots \pm n \pi \quad\left[\begin{array}{l} 
\\
N=10] \\
n=1 n t e g e r
\end{array}\right.
$$

and 18 a maximum when

$$
\sin (\theta)=\frac{ \pm n \lambda}{a}
$$

These maxima all have the same value and are equal to $N$. The maximum at $\sin (\theta)=\varnothing$ defines the main beam, the other maxima are called grating lobes and are generally undestrable.
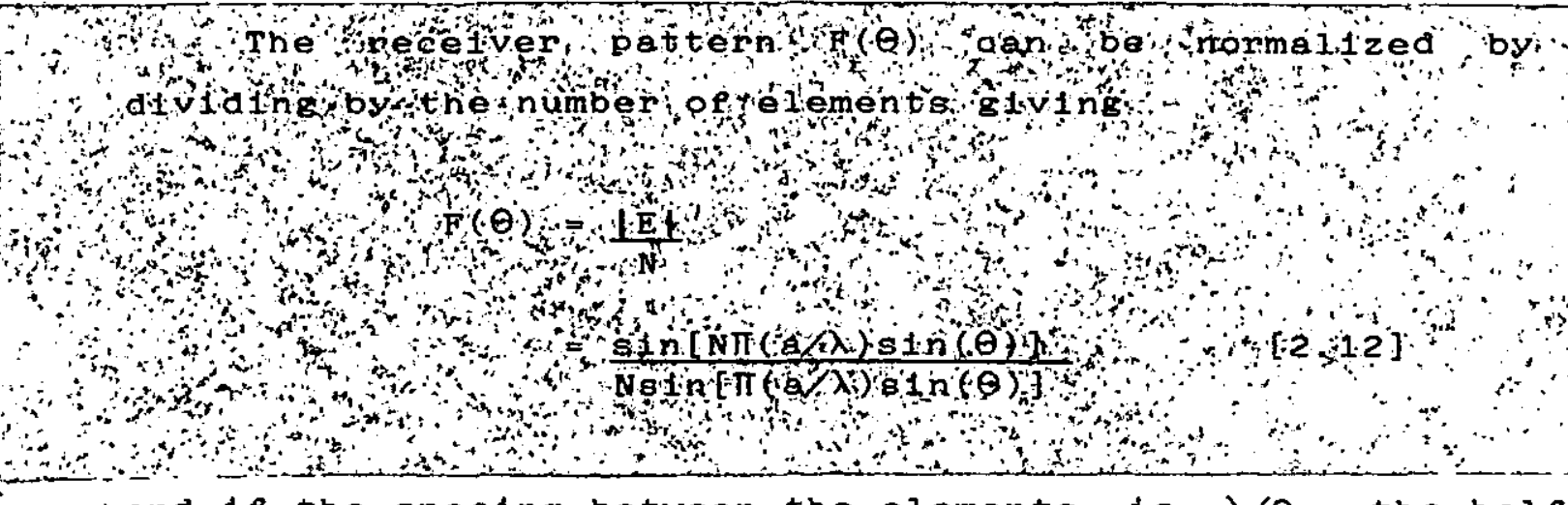
dividing pystie number op óleménts gleving

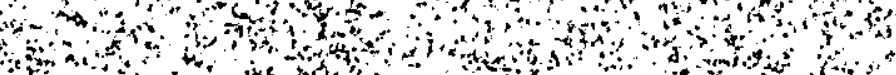

a

$\therefore$ ind

end

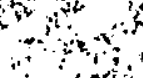

ind

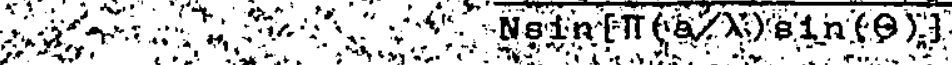

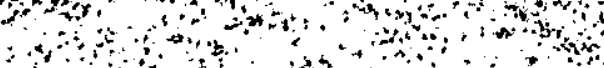

and if the spacing between the elements is $\lambda / 2$, the half power beemwldth $1 \mathrm{n}$ degrees is approximetely esueI to $102, \mathrm{~N}$. The first sidelobe, for large $N$ is 13.2 dB below the mein beam and the pattern 18 similar to a uniformly iliuminated 
continuous aperture.

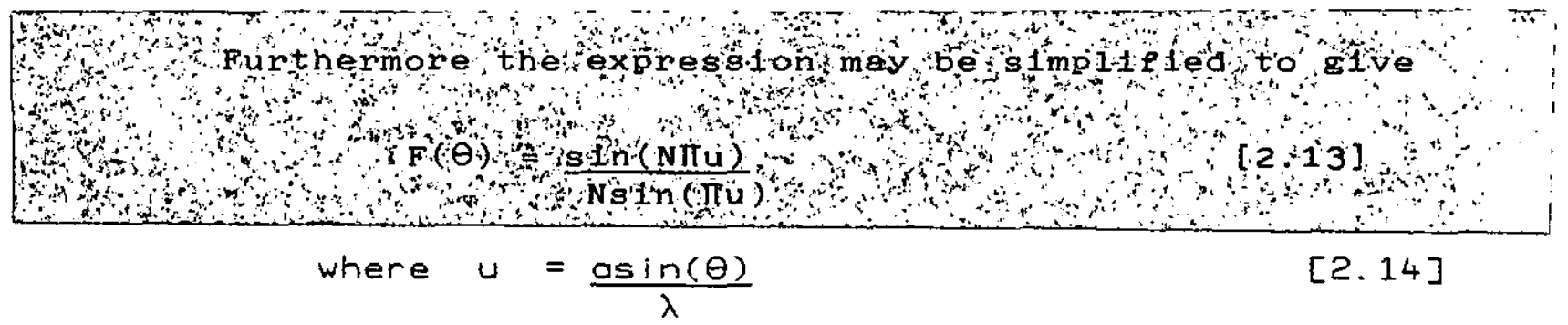

and a is the element spacing in a linear array of $N$ omnidirectional elements. The main beam is broadsicle but by varying the interelement phase shift, the beam position can be controlled to produce main beam scan.

\section{5 Resolution Achievable using Linear Arrous}

The conventional technique for achieving fine azimuth resolution has been that of radiating a narrow beam by the use of a large array. The resolving capability of the array is equal to the width of the beam at each range. Fig. 2.6 shows a conventional side-looking sonar. With an effective real array length of $d$. Assuming that the target is in the far-field of the array. the angular $3 \mathrm{db}$ beamwidth. $B$, is approximately equal to $\lambda / d$, where $\lambda$ is the wavelength of the transmitted signal. At a range of $R_{\text {a }}$ this gives a lateral resolution

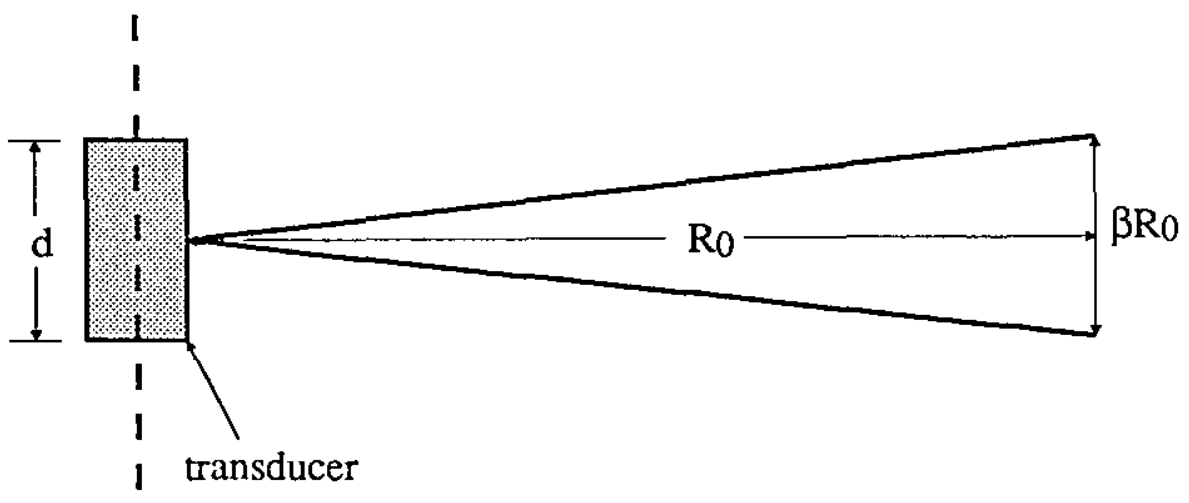

Fig. 2.6 Simple Side Looking Sonar 

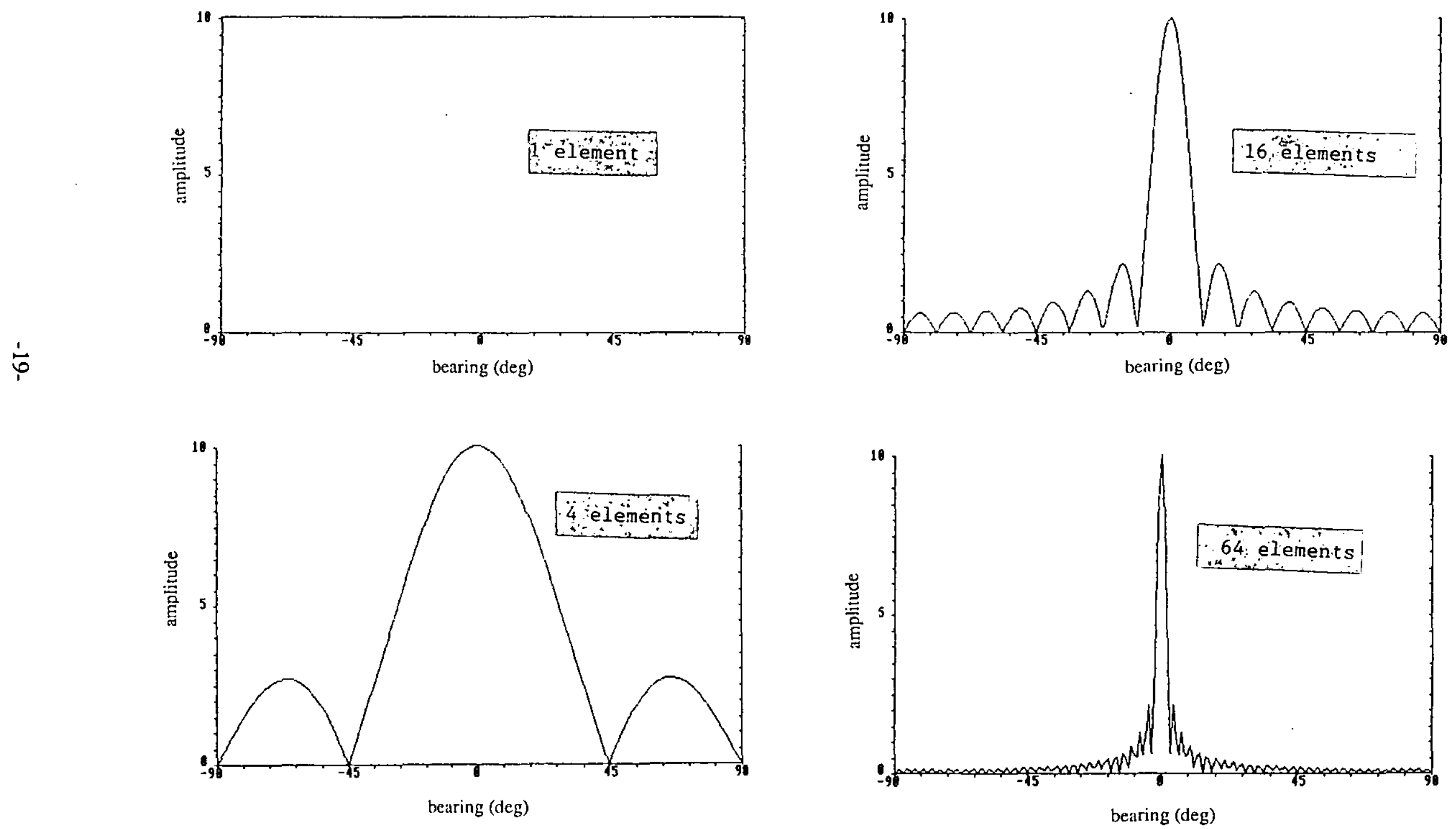

Fig. 2.7 Beam Pattern Variation with Array Size. 


$$
\delta \times=\lambda R_{\sigma} / d
$$

Figure 2.7 illustrotes the effects of varying the array size on the beam pattern. The element spacing is one wavelength and as the array size increases the beamwidth gets narrower, as shown in Fig. 2.7 (a) to Fig. 2.7 (d).

Thus the achievable resolution is determined by the array size, for a given range and wavelength. Obviously this resolution may be improved by using a larger array, but there are physical limitations to the maximum size. In addition to the practical difficulties in construction. Installation and operation of a large array. there is the limitation imposed by the near-field. The above expression for resolution applies only to the fartfield of the array. The beginning of the far-field occurs at a distance of $d^{2} / \lambda$ from the array and is shown in Fig. 2.8.

The finest resolution possible. therefore. is at the start of the farpfield. and is given by $B d^{2} / \lambda(a d)$.

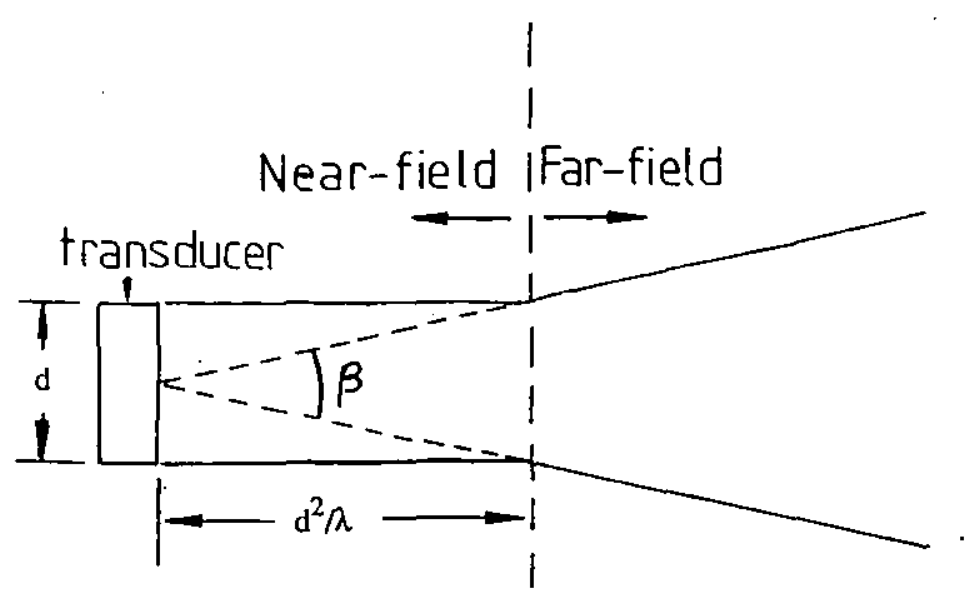

Fig. 2.8 Near-field and Far-field of a Transducer. 
Thus the higher the desired resolution, requiring o longer array, the worse the actual resolution becomes. A real array cannot provide the high azimuth resolution required by many applications olthough the real array has seen a marked increase in its use with the advent of techniques such as phase shifting. electronic steering and adapt Ive techniques.

\subsection{Resolution Achlevable using Sunthetic Arrays}

A real array radiates (and receives) from all the elements simultaneously and the combination of the signals transmitted (and received) result in an effective narrow main beam. The same effect can be obtained, under stationary conditions, by using a single transducer and storing the returns from successive transmissions. as the transducer moves to take up different positions in the synthetic array.

The major difference between the real and synthetic array is the path length difference at adjacent elements. For the real array. the phase difference ot adjacent elements from a target is proportional to the one-way distance. For the synthetic array, the phase difference is proportional to the two-way distance between the target and the array.

Thus the beamwidth of the synthetic array is half of the real array i.e.

$$
B=\frac{1}{2} \frac{\lambda}{L}
$$

where $L=$ synthetic aperture length. 
There are various techniques used to generate a synthetic aperture, the simplest being the use of a single transducer to both transmit and receive the signals. With this technique some method of switching is required to multiplex the transducer from the power amplifier when transmitting signals and the recelver electronics when recelving the returning echoes.

The use of two transducers eliminates the need of a "switch" with one transducer used for the transmit signals and the other to receive the echoes. The two transducers may be mounted together and moved along the aperture or one of the transducers may be stationary and the other moved.

The basic principle using a single transducer to transmit and receive is shown in Fig. 2.9. A pulsed transmitter drives a comparatively small transducer of width $d$, which is moved along a straight line. Received signals are range-gated and. for each range interval cor cell). the amplitude and phase history is stored. The processor assoclated with each range interval performs a continuous weighted summation of signals over a distance $D$ equal to the length of aperture which it is desired to synthesise. Thus the real aperture o essentially forms the element pattern for the synthesised aperture $D$.

Assuming the real radiation pottern of the real aperture $d$ can be approximated by a beam of angular width $\lambda / d$, then it is evident from the geometry of Fig. 2.9 that the maximum length of aperture which can be synthesised is proportional to range, that is:

$$
D_{a}=R \lambda / d
$$

When the transducer has moved a distance $\times$ along the 


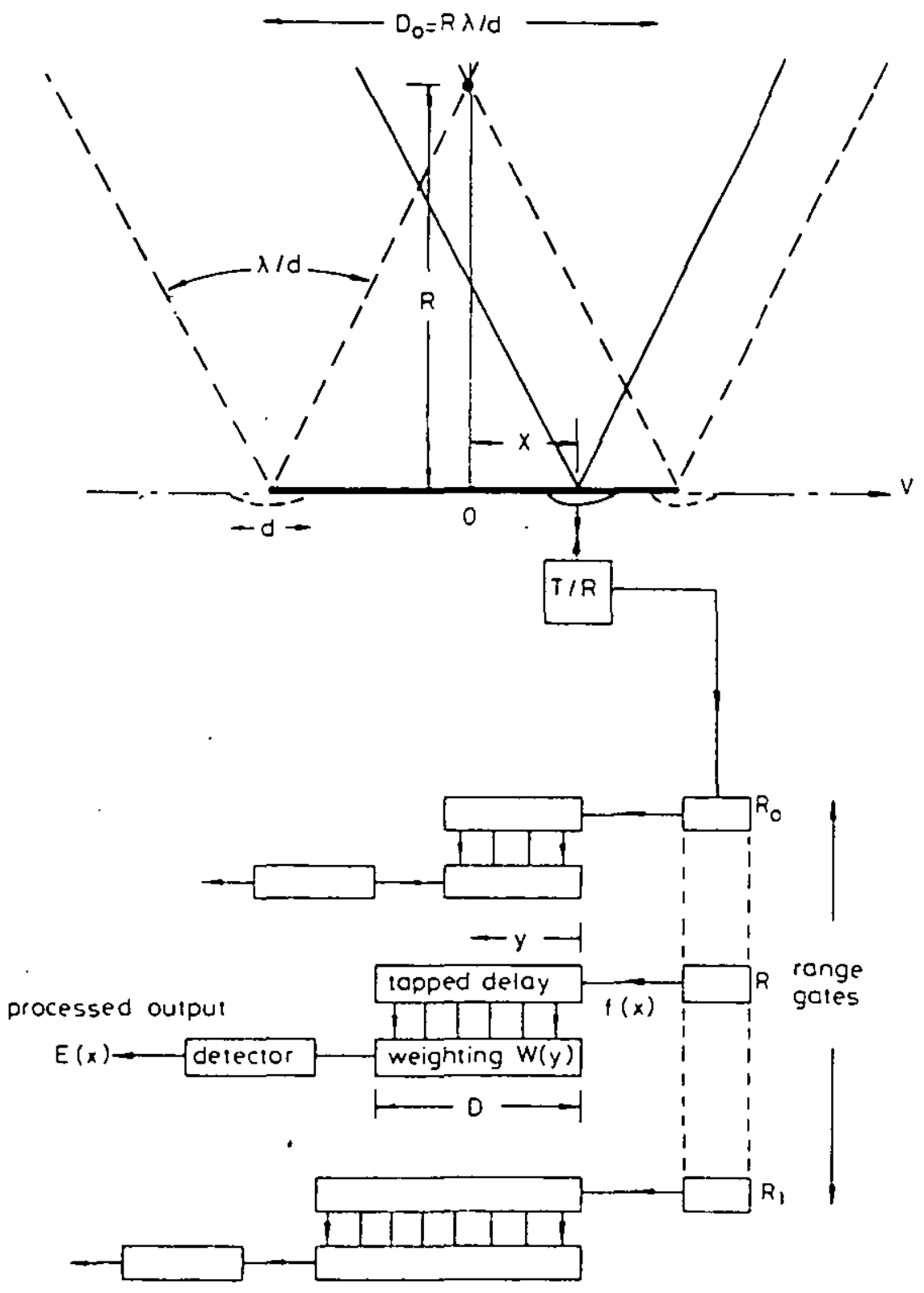

Fig. 2.9 Basic Principles of a Synthetic Array System. 
track, the additional path to the target

$$
\begin{aligned}
\sigma & =\sqrt{ }\left[R^{2}+x^{2}\right]-R \\
& \approx x^{2} / 2 R
\end{aligned}
$$

and hence the phase variation is

$$
\begin{aligned}
0 & =-4 \pi \delta / \lambda \\
& \approx-2 \pi x^{2} / R \lambda
\end{aligned}
$$

The received signal can therefore be represented by

$$
f(x)=A(x) \exp \left[-j 2 \pi x^{2} / R \lambda\right]
$$

where $A(x)$ includes the effects of the beam shape of the real aperture d. For a flat-topped beam and a point target:

$$
f(x)=\exp \left[-j 2 \pi x^{2} / R \lambda\right] \quad\left(-D_{x} / 2<x<D_{0} / 2\right) \quad[2.23]
$$

The hyperbolic nature of the phase history is shown in Fig. 2.10. The maximum phase excursion is directly proportional to range and inversely proportional to the square of the real aperture $d$.

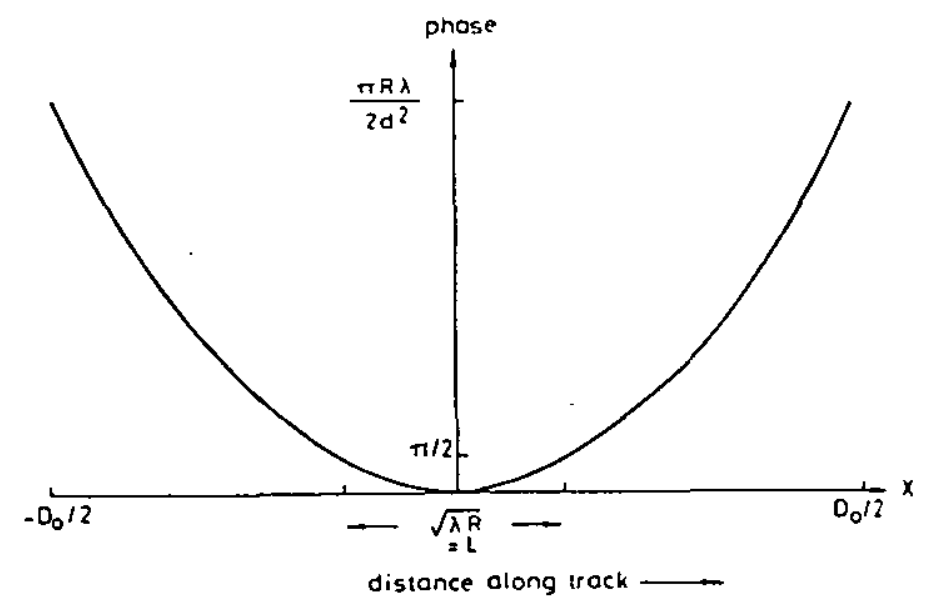

Fig. 2.10 Phase History of a Target. 
The distance along track for which the phase deviation is less than $\pi / 2$ is

$$
L=\sqrt{ }[R \lambda]
$$

which can be recognised as the fraction of the synthesised aperture having a far-field range just equal to $R$.

The processor performs a weighted summation of signals received over a distance $D$ which generally lies between $L$ and $D_{\alpha}$. Assuming a continuous sampling over this distance, then the processed output can be approximated by

$$
E(x)=\int W(y) f(x-y) d y
$$

where $W(y)$ represents the amplitude and phase weighting applied in the processor. Essentlally, the processor associated with each range interval performs a convolution between the range-gated received signal $f(x)$ and the stored weighting function $W(y)$.

There are two types of synthetic arrays: the unfocussed array and the focussed array. and the cholce as to which is used has implications on the processing involved and the achievable resolution.

\subsubsection{Unfocussed Sunthet ic Arrous}

The simpler of the synthetic aperture technique is that which generates an unfocussed array. In this case the signals received at the array positions are integrated with no. phase adjustment. This imposes a maximum length upon the synthetic aperture which can be generated. The maximum length occurs when the round-trip distance from a target to the centre of the array differs by $\lambda / 4$ from the 
round-trip distance between the target and the extremeties of the array [19]. This restriction is necessary due to the fact that since there is no phase adjustment of the signals received at each element position prior to summing. the received signals outside this interval are largely self-cancelling due to the rapid phase variation. This type of array is only used for far-field operation.

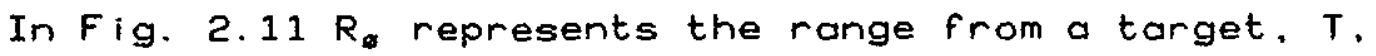
to the centre of the array. Leff represents the maximum synthetic aperture length for which the distance from the target to the extremities of the array does not exceed $R_{\mathrm{g}}+\lambda / 8$. From this

$$
\left(R_{8}+\lambda / 8\right)^{2}=\left(L_{e f f}\right)^{2 / 4}+R_{\infty}^{2} \quad[2.26]
$$

Hence

$$
L_{\text {eff }}=\sqrt{ }\left(R_{\boldsymbol{g}} \lambda\right)
$$

(assuming that $\lambda^{2} / 16$ is small compared with $\lambda / 16$ )

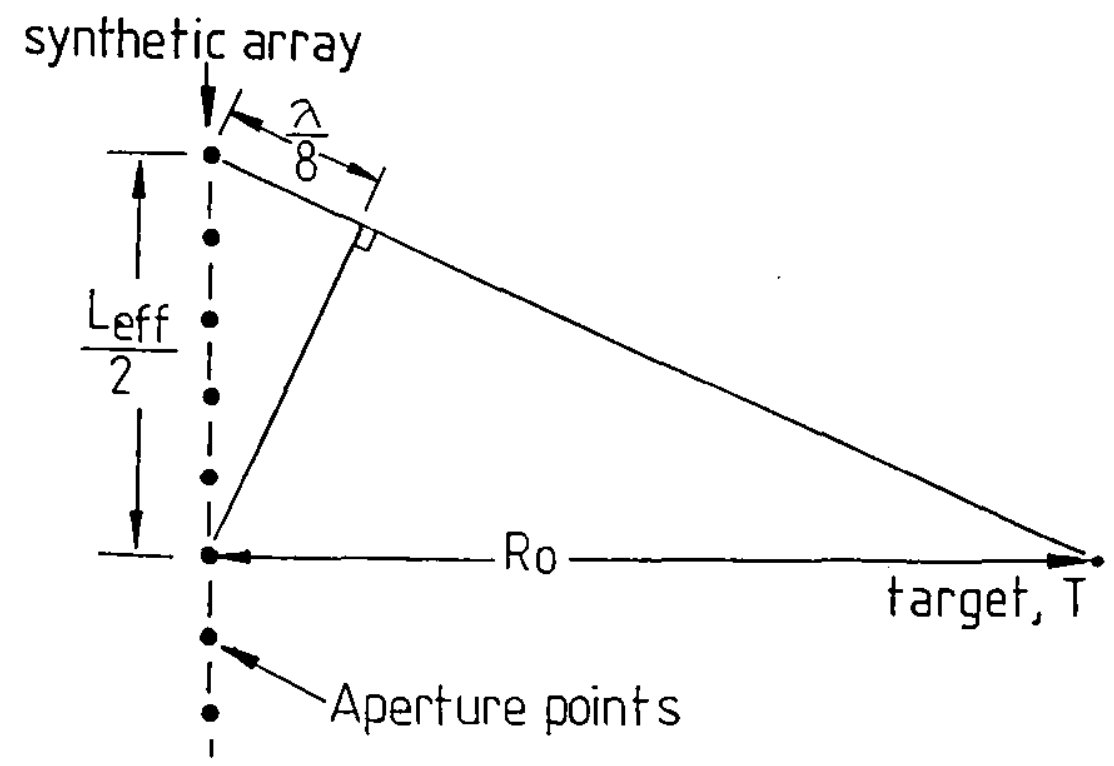

Eig 2.11 Unfocussed Synthetic Array. 
The resolution of an optimised unfocussed synthetic array is

$$
\delta \times_{\text {unfocussed }}=\frac{\lambda R_{\text {g }}}{2 L_{\text {eff }}}
$$

Subst ituting for $L_{\text {eff }}$ from equation $[2.27]$ gives

$$
\delta \times_{\text {unfocussed }}=\frac{\sqrt{ }\left(\lambda R_{a}\right)}{2}
$$

The corresponding angular beamwidth is

$$
\Phi_{2}=\lambda / 2 L_{\text {eff }}=\sqrt{ }(\lambda / 4 R) \quad[2.30]
$$

This is half the normal beamwidth of an aperture of length $L$, due to the two-way phase effect. In practical systems, the welghting function $W(y)$ includes appropriate amplitude weights which, together with any amplitude weighting due to the beam shope of the real aperture $d$, can be chosen to improve the sidelobe response.

\subsubsection{Focussed Sunthetic Arrays}

A dramatic increase in resolution is obtained by operating in the focussed mode. where the weighting function includes phase terms which compensate for the parabolic variation in signal phase. Thus for each range gate the weighting function is

$$
W(y)=\exp \left[J \pi(D-2 y)^{2} / 2 R \lambda\right]
$$

plus any amplitude weighting which is introduced to control the sidelobes.

The maximum length of the array is now defined by the time for which the target is illuminated by the beam of the 
real aperture $d$ as the transducer moves along the synthetic aperture, i.e. the beamwidth at the range of interest.

Thus at range $R_{\text {a }}$, the maximum synthetic array length is

$$
L_{\text {max }}=\frac{\lambda R_{a}}{d}
$$

Hence the azimuth resolution for a focussed synthetic aperture is

$$
\delta x_{f o c u s s e d}=\frac{\lambda R_{\alpha}}{2 L_{\max }}
$$

Substituting for $L_{\text {max }}$ from equation $[2.32]$ gives

$$
\delta x_{\text {focussed }}=\frac{d}{2}
$$

Note that the theoretical resolution for a focussed array is independent of range and wavelength and directly proportional to the dimensions of the transducer. Fig. 2. 12 illustrates the resolution achlevable for the various types of arrays discussed. The possibility exists for generating very long synthetic apertures, thus giving the potential for high resolution.

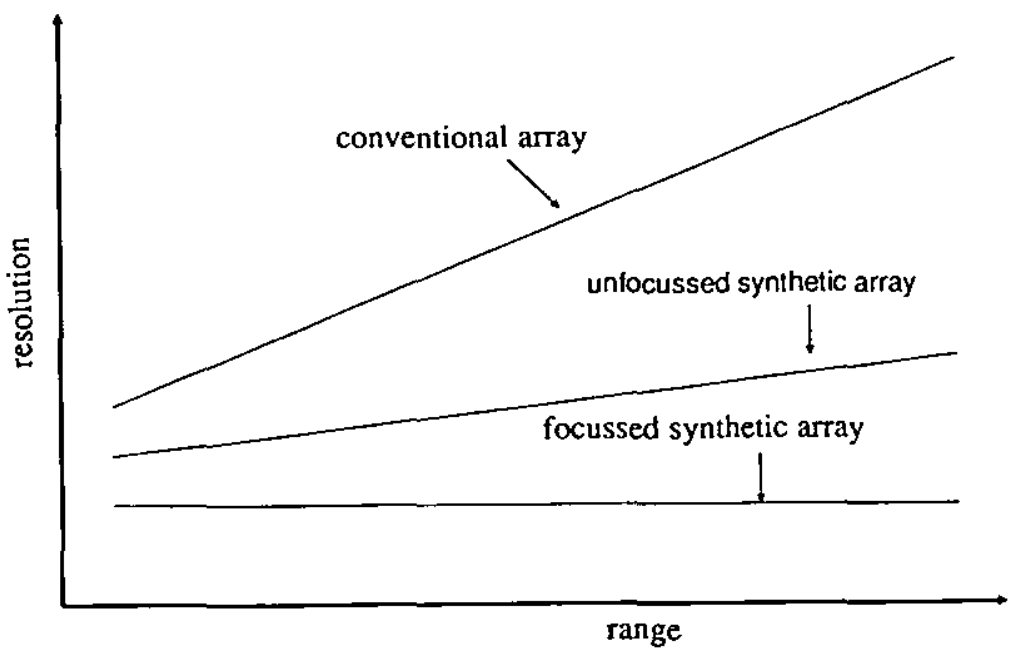

Fig.2.12 Comparison of Azimuth Resolution Achievable with Conventional and Synthetic Arrays. 
The apparent anomaly that the along-track resolution is proportional to d rather than inversely proportional to $d$ as in conventional arrays is resolved when it is recognised that choosing d small enables a large value of synthetic aperture to be realized. The penalty pald for increasing the resolution is that more processing power is required.

The waveform of the processed output may be obtained by substituting eqns. [2.31] and [2.22] into eqn. [2.25]. For the case of uniform amplitude weighting and an optimised system $\left(L=D_{\alpha}\right)$. the processed output signal becomes:

$$
\begin{aligned}
E(x)=\int \exp \left(j \pi\left(D_{0}-2 y\right)^{2} / 2 R \lambda\right) & \\
& \quad \exp \left(-j 2 \pi(x-y)^{2} / R \lambda\right) d y \quad[2.35] \\
= & \exp \left[j \pi\left(D_{0}^{2}-4 x^{2}\right) / 2 R \lambda\right] \\
& \quad \exp \left[-j 2 \pi y\left(D_{0}-2 x\right) / R \lambda\right] d y[2.36]
\end{aligned}
$$

where the approprlate integration limits are

$$
y=0 \text { to }\left(D_{x} / 2+x\right) \text { for }-D_{0} / 2<x<D_{0} / 2
$$

The phase term represents a I inear variation with $y$. with a slope proportional to the olong track distance $x$. Thus

$$
E(x)=\frac{\sin \left[\pi\left(D_{0}-2 x\right)\left(D_{0}+2 x\right) / 2 R \lambda\right]}{\left[\pi\left(D_{0}-2 x\right) / R \lambda\right]} \begin{gathered}
\text { where }-0_{0} / 2<x<0_{s} / 2 \\
\text { where }
\end{gathered}
$$

This can be written more compactly by shifting the origin to the position of peak response by writing:

$$
x=x-D_{x} / 2
$$

Then noting that $D_{0}=R \lambda / d$, the expression for the 
processor output becomes:

$$
E(x)=\frac{D_{0} \sin \left[2 \pi(x / d)\left(1-\left|x / D_{a}\right|\right)\right]}{[2 \pi(x / d)]}-D_{0}<x<D_{0}[2.40]
$$

As illustrated in Fig. 2.13, the peak output occurs at $x=$ $\theta\left(x=D_{0} / 2\right)$. With an amplitude value of $D_{0}$ and $a-3 d B$ width of $d / 2$. The waveform is substantially of $\sin (x) / x$ shape but the periods of the sidelobes increases as the distance from the centre peak increases due to the reduction in the length of the stored signal. The total duration of the output signal is $2 \mathrm{D}_{\sigma}$. Sidelobes can be improved by the introduction of amplitude taper into the weighting function.

For a non optimised focussed synthetic aperture system $\left(D<D_{\infty}\right)$ the peak signal is reduced to $D$, and the beamwidth increases to $\left(\mathrm{dD}_{\alpha} / 2 \mathrm{D}\right)$.

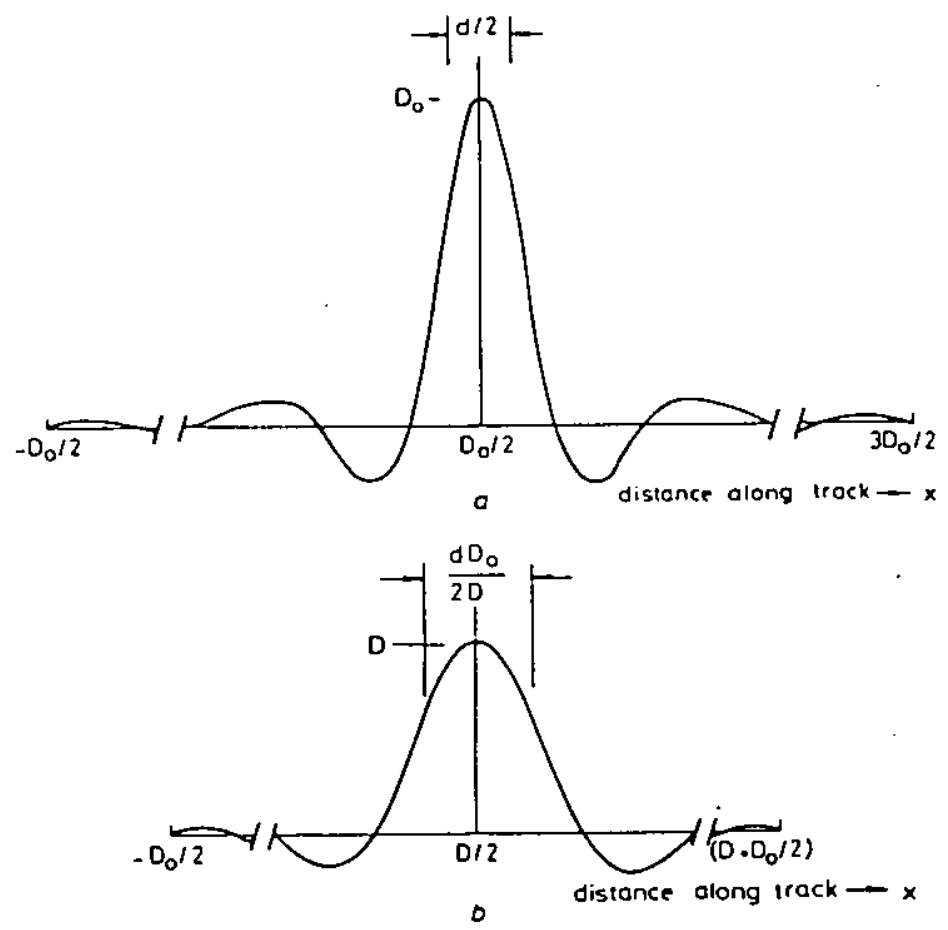

Fig, 2.13 Processed Output of a Synthetic Aperture System. 


\subsection{Grating Lobes}

If the spacing between elements in a real array is $\lambda / 2$, the first grating lobe does not appear in real space since $\sin (\theta)>1$. Grating lobes appear at $\pm 90^{\circ}$ when the element spacing $a=\lambda$. For a non-scanning array this condition is usually satisfactory for the prevention of grating lobes. The above expression applies to isotropic elements, but practical elements that are designed to maximise the radiation at $\theta=0^{\circ}$. generally have negligible radiation in the direction $\theta= \pm 90^{\circ}$. Thus the effects of a realistic element pattern is to suppress the grating lobes at $\pm 90^{\circ}$ and for this reason element spacing equal to one wavelength can be tolerated for a non scanning array.

Large spacings of $a / \lambda$ will allow the development of grating lobes whose angular positions are given by

$$
\frac{\mathrm{a}}{\lambda}=\frac{n}{\left(\sin \left(\theta_{0}\right)-\sin (\Psi)\right.}
$$

$$
\begin{aligned}
\text { where } \theta_{0} & =\text { main beam angle } \\
\text { and } \Psi & =\text { angle of nth grating lobe. }
\end{aligned}
$$

Grating lobes can be reduced by ensuring that an element radiation pattern null occurs at the position of the grating lobe. When the element spacing is $\lambda / 2$, destructive interference of the signals in the direction $\theta$ $= \pm 90^{\circ}$ means that no energy is transmitted (or received) in these directions.

A similar condition for grating lobes in synthetic aperture systems is that the distance travelled by the transducer between pulse transmissions should be less than $\lambda / 2$. The factor $1 / 2$ appears due to the two way propagation 
path from the element to the target and back as compared with the one way path of a conventional array. When a directive element is used the spacing necessary between pulse transmissions can be greater than $\lambda / 2$ and by making the angular locations of the first grating lobes coincide with the first null of the element pattern grating lobes can be attenuated.

The position of the grating lobe in the synthetic aperture system is

$$
\Psi=\frac{\lambda}{2 s}
$$

where $s$ = spacing between transmissions.

The position of the first null of the element pattern is approximately equal to $\lambda / d$ where $d=$ width of element. Since the grating lobe angle must be equal to or greater than the position of the null, to avoid grating lobes the following condition is obtained

$$
2 \frac{\lambda}{s} \geqslant \frac{\lambda}{d}
$$

thus

$$
s \leqslant \frac{d}{2}
$$

In a synthetic aperture system there is no destructive interference effect in the direction $\theta= \pm 90^{\circ}$ and some energy may be transmitted in these directions even when the spacing is $\lambda / 2$.

\section{8 The Sonar Equation}

The many phenomena and effects peculiar to underwater acoustics produce a variety of quantitative effects on 
the design and operation of underwater sonar equipment. These diverse effects can be conventently and logically grouped together in a small number of quantities called the sonar parameters, which, in turn are related by the sonar equations. These equations are the working relationships that tie together the effects of the medium, the target and the equipment.

The relationship of these quantities can be best 11Iustrated by considering a simple active soner system. We shall first derive an expression for the relationship between the various parameters and then discuss some of the parameters in more detall.

A transmitter produces a source level of SL dectbels reference. to a standard level at a unit distance of $1 \mathrm{~m}$ on 1ts ax1s. When the radiated slgnal reaches the targot, 1ts level will be reduced by the transmission $10 s 3$, and become SL - TL. On reflection or scattering by the' target of target strength TS, the reflected gignal will be SL - TL + $\mathrm{TS}$ at a distance of $1 \mathrm{~m}$ from the acoustic cantre of the target in the direction towards the source. In traveling back towards the source, the signal is again attenuated by the transmission loss and becomes SL - 2TL + TS. This is the echo level at the trangducer.

If the background is lsotroolc noise rather than. reverberation, then the background level can be expressed as NL. This level is reduced by the directivity index of the transducer and the relative nolse power becomes NL DI. Since the axis of the transducer is pointing in the directicn from which the echo is coming. the relative echo power is unaffected by the transducer directivity. The signal to nolse ratio at the transducer therefore is 


$$
S N R=S L-2 T L+T S-(N L-D I) .
$$

Some of these quantities will be discussed in more detail in the following section.

\section{8.1 The Definition of Source Level}

The acoustic intensity $I_{0}$ at a distance $1 \mathrm{~m}$ from an omnidirectional source radiating a power of $W$ acoustic watts is $I_{0}=W / 4 \pi$. If the source possesses directional properties more energy can be transmitted along a specified direction and the signal strength increases by the directivity factor. DF. The acoustic intensity of a directional source ot a distance of $1 \mathrm{~m}$ is therefore given by $I_{0}=W / 4 \pi \times D F$. The source level is defined as

$$
S L=10 \cdot \log _{10}\left(I_{0}\right)
$$

Thus

$$
\begin{aligned}
& S L=-11+10 \cdot \log _{10}(W)+D I \\
& \text { in dBs relative to } 1 W / \mathrm{m}^{2} \\
& \text { where the directivity index } \\
& D I=10 \cdot \log _{1}(D F)
\end{aligned}
$$

\section{8.2 The Definition of Torget Strength}

The target strength TS of a reflecting body is defined by the expression

$$
T S=10 \cdot \log _{10}\left(I_{5} / I_{i}\right)
$$

where $I_{s}$ and $I_{i}$ represent the reflected or scattered and incident intensities respectively. For complex targets. the TS is difficult to calculate or measure but for a simple, smooth spherical rigid target well removed from the 
surface or bottom 1 t $1 \mathrm{~s}$ less difficult. There $1 \mathrm{~s}$ only a simpie echo and if it assumed that the sphere radiates equaliy in all directions, and that 1 ts range from the source is larger compared with the wavelength of the signal then the calculation 1 s as follows. The source level is defined as the source intensity at $1 \mathrm{~m}$ from the the source. If the sound field at the target has intensity I and the sphere has radius a metres, then the acoustic power incident upon the target $13 \pi a^{2} I$. This power 1 s reradiated equaliy in all directions so that at $1 \mathrm{~m}$ from the centre of the sphere the acoustic intensity $1 \mathrm{~s} \pi \mathrm{a}^{2} I / 4 \pi$ which $1 \mathrm{~s}$ equal to $a^{2} I / 4$. This is the actual source strength of the target. The source strength relative to the intensity of the Incident field is therefore equal to $\mathrm{a}^{2} / 4$. Thus the target strength can be written as

$$
T S=20 \cdot \log _{1 \cdot(a / 2)} \mathrm{dB}
$$

for a rigid sphere whose radius is large compared to the wavelength: A unit target having a target strength of $\varnothing \mathrm{dB}$ w11l be a sphere of radius $2 \mathrm{~m}$ but the target strength of an object with an 1rregular shape depends not only on 1 ts size tut 1ts orientation as well.

\subsubsection{The Definttion of Transmission Loss}

Transmisston ioss indicates the amount of weakenfing of the signal between a reference point and a point at a distance in the water. If $I_{\text {. }}$ is the intensity of sound at the reference point located $1 \mathrm{~m}$ from the source and $I_{1}$ Is the intensity at a distant point, then the transmisaion loss, TL, between the source and the distant point is defined as

$$
T L=10.108=0 \frac{\left(I_{8}\right)}{I_{1}}
$$


Transmission loss is the sum of two quantities, spreading and attenuation. Spreading loss is the weakening of the signal as it spreads outwards from the source to occupy a larger and larger area and varles with the log of the range. Attenuation loss includes the effects due to absorption, scattering and leakages out of the sound channel and varies linearly with range. Spherical and cylinderical spreading will be considered first.

\subsubsection{Transmission Loss due to Spherical Spreading}

Consider a small source in a homogeneous, unbounded and lossless medium, as shown on Fig. 2.14(a). The power generated by the source is radiated equally in all directions, so as to be equally distributed over the surface of a sphere surrounding the source. Since there is no loss in the medium. the power crossing oll such spheres must be the same. The sound intensity. I at a radial distance $R$, from the source, $S$, of power $P$ is

$$
I=P / 4 \pi R^{2}
$$

If $R_{1}=1 \mathrm{~m}$. and substituting eqtn. [2.52] into eqtn. [2.51] for $I$, the transmission lass at range $R_{2}$ is

$$
\begin{aligned}
T L & =10 \cdot \log _{10}\left(R_{2}^{2}\right) \\
& =20 \cdot \log _{10}\left(R_{2}\right)
\end{aligned}
$$

\subsubsection{Transmission Loss due to Cylindrical Spreading}

When the medium is bounded by a parallel upper and 


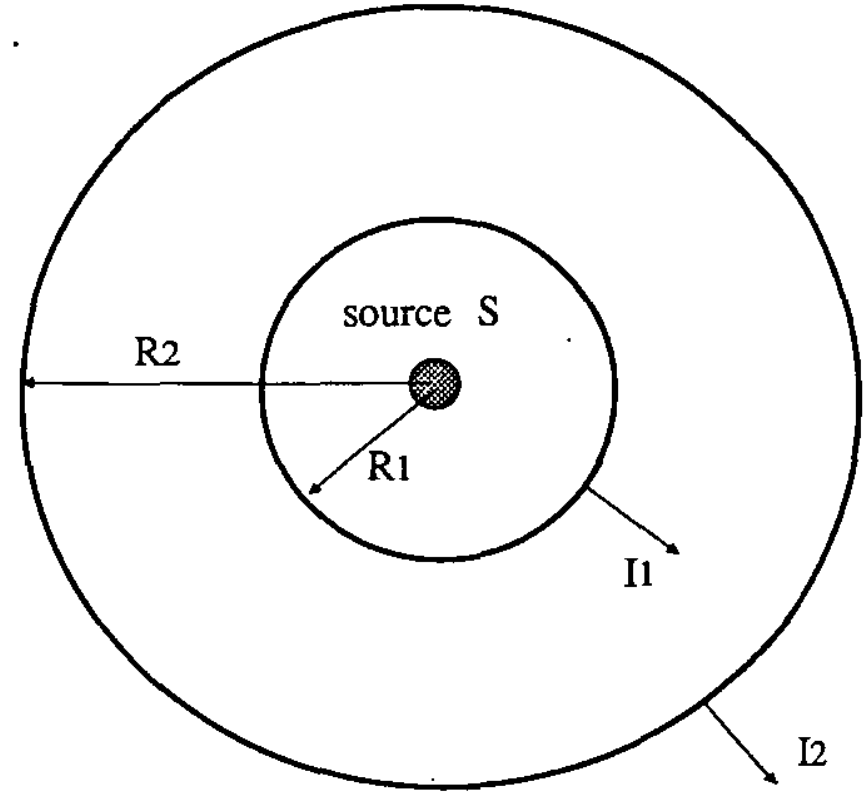

Fig, 2.14(a) Spherical Spreading in an Unbounded Medium.

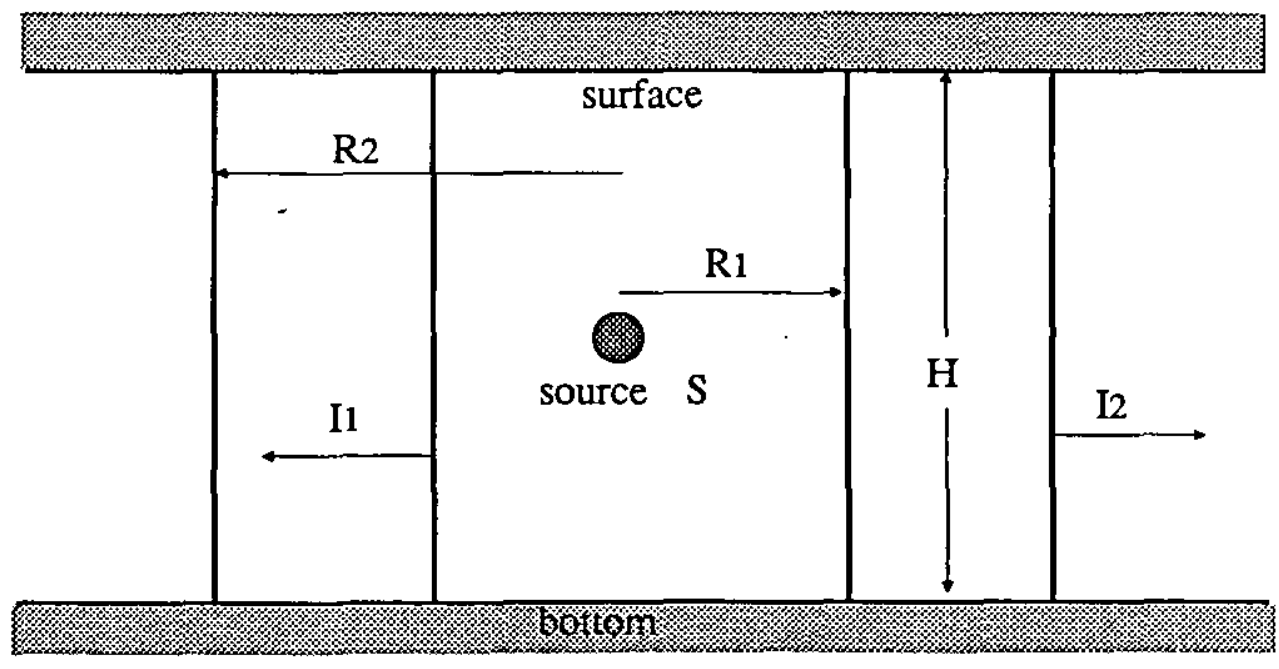

Eig.2.14(b) Cylindrical Spreading in Shallow Water. 
lower Iimits (as the shallow part of the sea is by the surface and bottom), the spreading is no longer spherical. as illustrated in Fig. 2.14(b). Beyond a certain range the power radiated by the source is distributed over the surface of a cylinder. The intensity at radial distance $R$ from the source, is now

$$
I=P /(2 \pi R H)
$$

where $H$ is the sea depth.

Again taking $R_{1}=1 \mathrm{~m}$, the transmission loss at range $R_{2}$ becomes

$$
T L=10 \cdot \log _{1}\left(R_{2}\right)
$$

\subsection{3 Transmission Loss due to Absorption}

Absorption is the loss in energy due to the conversion of acoustic energy into heat as the signal travels through the water. The absorption loss is a function of frequency of the signal, the salinity and depth of water. Figure 2. 15 Illustrates the variation of absorption with frequency in fresh and sea water.

\footnotetext{
Taking into account the absorption loss, the expression for transmission loss with spherical spreading now becomes
}

$$
T L=20 . \log _{10}(R)+\alpha R
$$

where $\alpha$ is the coefficient of absorption in $\mathrm{dB} / \mathrm{m}$ and $g$ ives a good indication of the losses in most general cases. 


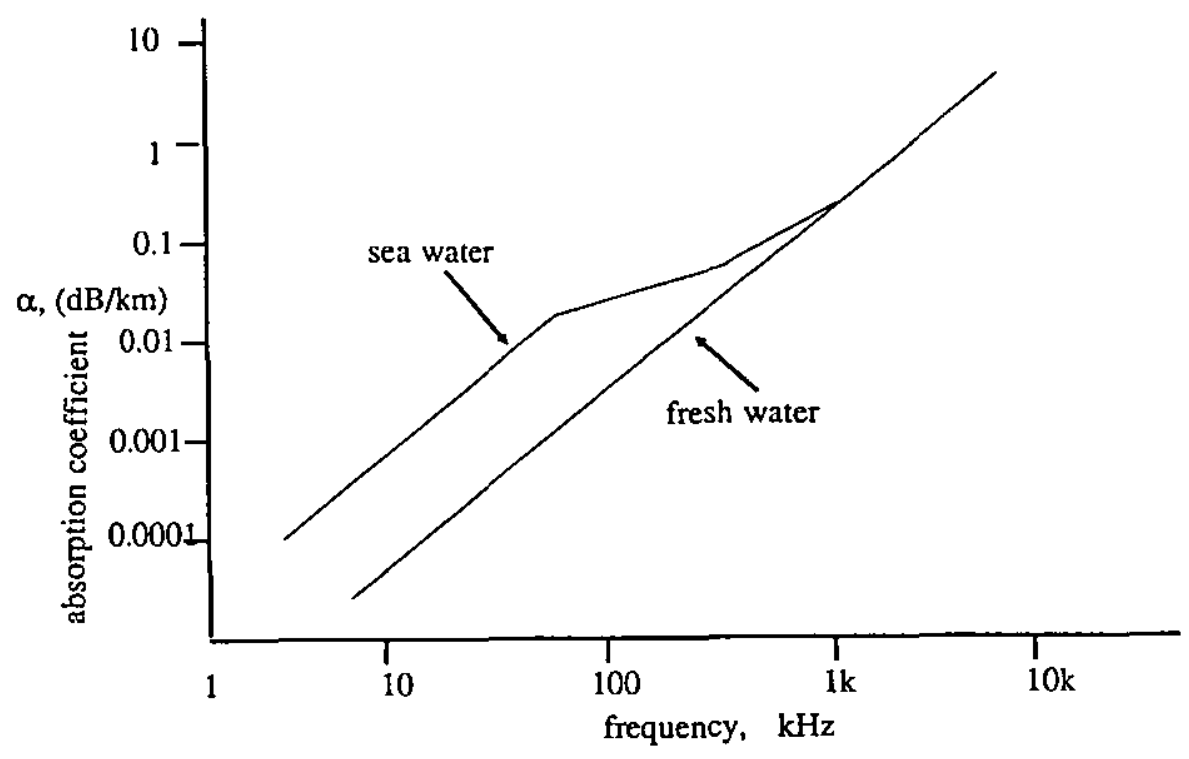

EIG. 2.15 Variation of Absorption Loss with Frequency.

\subsection{Restrictions in the Underwater use of Sunthet ic Aperture Sonar Systems}

The obvious value of a synthetic aperture arises from the ability to achieve good azimuthal resolution without the need to deplay a very long receiving array. with good heading control. a simple system consisting of a single transmitter and a single recelver could provide the same resolution as a long array. and in addition, be free of the speed and manoeuvring constraints imposed by a large array. Such a system might find ready opplication in detailed ocean bottom mapping survey. 
Difficulties facing an acoustic attempt at forming a successful synthetic aperture were apparent from the start and have restricted the application of the synthetic aperture technique to underwater applications. Motion irregularities and media turbulence, causing phase errors greater then $\lambda / 4$, [56] must be corrected to generate a synthetic operture. The relatively slow acoustic propogation velocity in water. Implies a low pulse repetition frequency. With the following consequences:

(i) Large amounts of irregular and unknown motion in the transducer path can occur between pulses.

(ii) The multipath pattern of propagation through the ocean can exhibit signiflcant instability in this period.

(iii) The coherent combination of returns from successive pulses may be 1 imited to a few pulses thereby restricting the benefits of a synthetic aperture.

(iv) For targets at large ranges, a range ambiguity problem may arise. If a succeeding pulse is transmitted before the echoes from the preceding pulse have been completely received.

The following section considers some of these problems and discusses methods to overcome them.

\subsection{Limitations due to the Low Propagation Velocity of Sound in Water}

One of the main reasons for the lack of acceptance of the synthetic aperture technlque in sonar has been the I Imited unambiguous range. This ambiguity arises due to the low propagation velocity of sound in the sea. Before the next pulse can be transmitted, echoes from the maximum range of interest must be received. This 
restriction limits the transducer motion velocity and hence the mapping rate.

This limitation can be shown by considering the time of propagation of the pulsed signal and the time it takes for the transducer to move to the next location in the synthetic array. The time taken to travel between adjacent element positions is $t$, where

$$
t=\frac{\delta x}{v}
$$

where $\nu$ is the sonar platform velocity and $\delta x$ is the cross-range resolution.

The propagation of the signal over the swathe width. $W(W$ $=R_{\text {max }}-R_{\text {min }}$ ) must occur during the same $t$ ime interval. in order to avoid ambiguities, therefore

$$
t \geqslant \frac{2 W}{c}
$$

From equations [2.59] and [2.58].

$$
W \leqslant \frac{c \delta x}{2 v}
$$

or that the swathe width depends upon the ratio $c / v$.

This was a serlous limitation. however, Cutrona [18] proposed a multiple beam system to overcome this ambiguity barrier. Hughes [19] compared the area mapping rates of a conventional single element. search slde-looking sonar system with a synthetic aperture sonar system with 17 transducers and concluded that the synthetic aperture system was 150 times faster.

The mapping rate can also be increased by the use 
of multiple frequencies [20]. One approach is to transmit a number of contiguous elevation beams each employing a different frequency and covering a part of the total range swathe. Fig. 2.16 illustrates this concept for a 2 frequency case. The time between sucessive transmissions is now determined by the range subswath covered by each elevation beam. The resultant mapping rate increases I inearly with the number of frequencies employed.

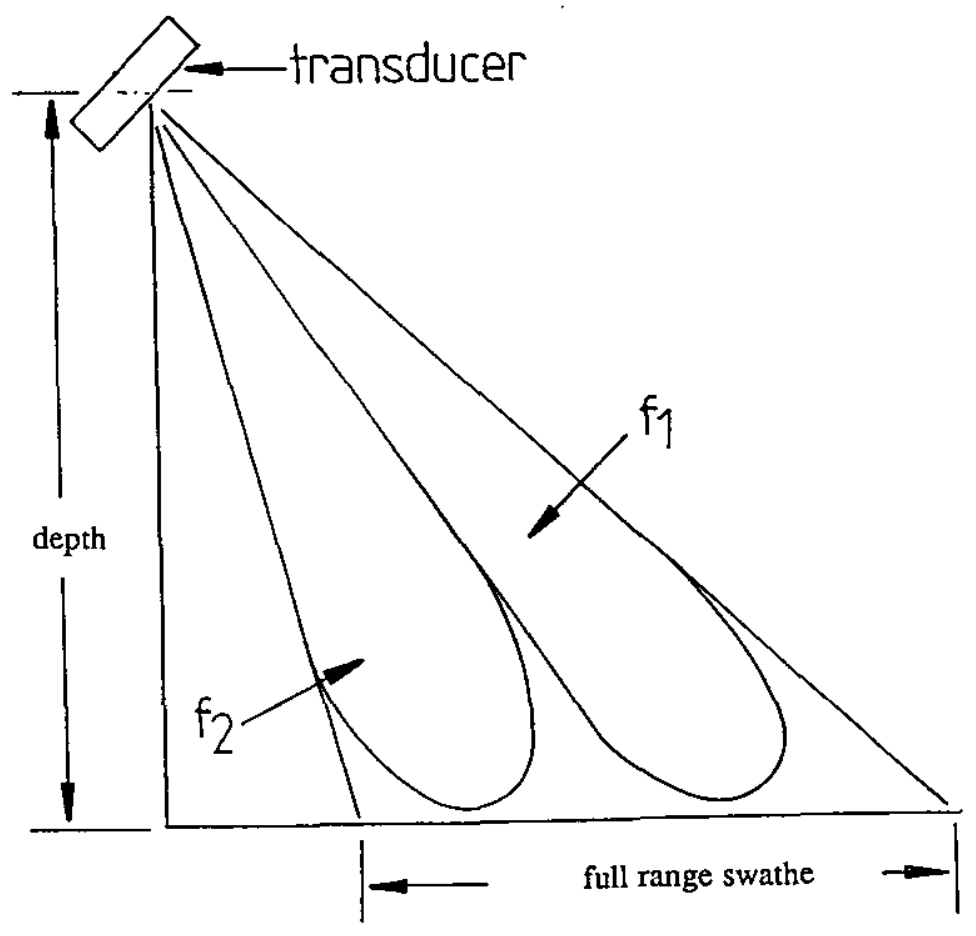

Fig. 2.16 Using_A Two Erequency System to.Increase the Mapping Rate

\subsubsection{The Effects of Media Turbulence}

Turbulence of the propagation media is also a factor that restricted the application of the synthetic aperture technique to underwater systems. Turbulence caused by inhomogeneities of salinity and temperature. for example, can lead to degradation of the reconstructed 
Image by the introduction of phase errors [47.50.56]. These phase errors must be less than $\pi / 2$ for the synthetic aperture technique to be applied sucessfully $[51.55]$.

Sato et al [26,27] have applied a technique to underwater imaging. first applied by Goodman et al [28], of introducing a reference signal in order to cancel the effects of turbulence of the media to obtain images in the same time as conventional synthetic aperture sonar systems.

\subsubsection{Transducer Motion Errors}

The successful application of the synthetic aperture technique relies upon the accurate knowledge of the transducer motion, as the transducer moves to take up consecutive positions in the aperture. If the transducer position or motion is not known accurately. or there are unknown motions in the transducer path, phase errors will be Introduced which will seriously degrade the image [20.41.45.49]. Methods for correcting motion phase errors are complex involving inertial navigation systems, and the measurement of three I Inear accelerations and three angular velocities.

\subsubsection{Image Degradation due to Target Motion}

Previous discussions have assumed that the isonified objects remain stationary during the time taken to generate the synthetic aperture. If however the target is not stationary it will result in a blurred and attenuated image of the target [21.52.53] as depicted in Fig. 2.17.

Any target movement during the time taken to traverse 
the synthetic aperture will destroy the phase coherence and in general results in a reduction in the SNR. with the

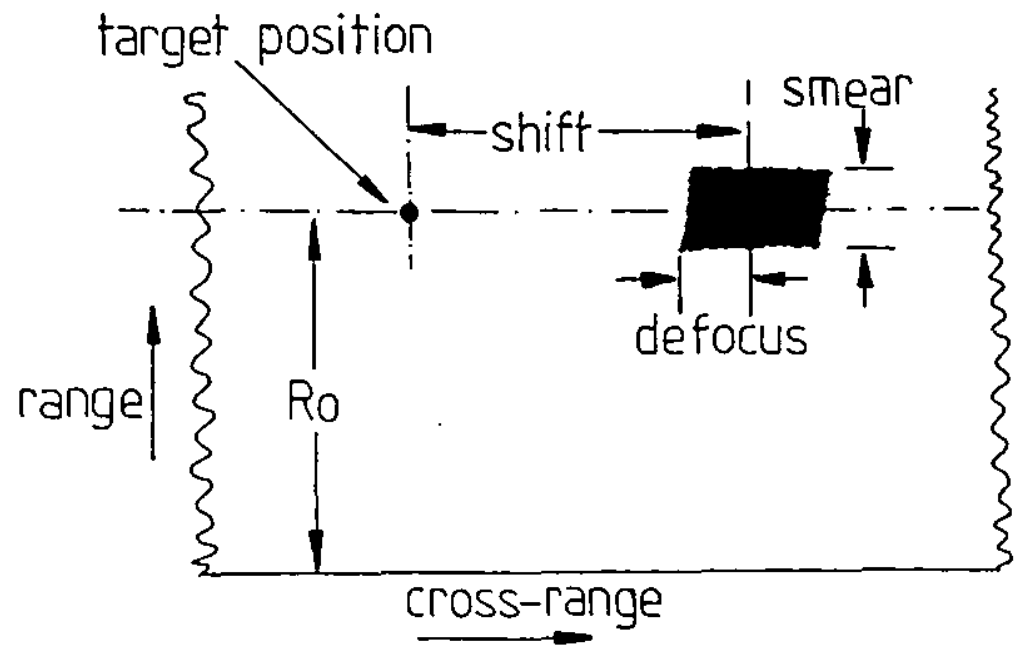

Fig, 2.17 Reconstructed Image of a Moving_Target.

image being smeared over a number of resolution cells. In order to provide better images in these circumstances, the length of aperture which is coherently synthesized is sometimes reduced, but a number of such overlapping apertures are added together non-coherently.

\subsection{Radar and Sonar Synthetic Aperture Sustems}

In synthetic aperture radar systems, high azimuth resolution is obtained by the coherent summing of the received signal, as the antenna moves along a known path. The relative motion between the antenna and the target causes a frequency shift, due to the doppler effect. In synthetic aperture sonar systems however. It is the change in the relative position between the transducer and the target that cause variations in the phase of the received signals. This phase variation across the aperture is used in the reconstruction process. 


\section{SIGNAL PROCESSING TECHNIQUES}

\section{1 Introduct ion}

A two-dimensional image of a radar or sonar mapped area can be produced by coherently processing signals received by the synthetic aperture. The range of a target is determined by the delay of the received signal, and the position along the azimuth or cross-range axis is determined by the doppler frequency or the phase variation. of the return signal from the target. The total phase and amplitude history of a target must be collected before signal processing can commence. The optical and digital methods of signal processing techniques will be discussed. The optical method requiring photographic equipment [8,9] is quite mature and numerous processed images have been reported, especially in terrain mapping synthetic aperture radar. Digital methods utilize computers and offer the potential of near real-time imaging $[15,16,17]$.

\section{3.? Optical Signal Processing}

To simplify the discussion we shall consider a single target geometry for a synthetic aperture radar system, but the arguments can just as easily be applied to sonar and ultrasound imaging systems.

The elevation view of a synthetic aperture radar system at an altitude of $H$ is shown in Fig. 3.1(a). The radar antenna beam of width $\theta e$ intercepts the ground at 


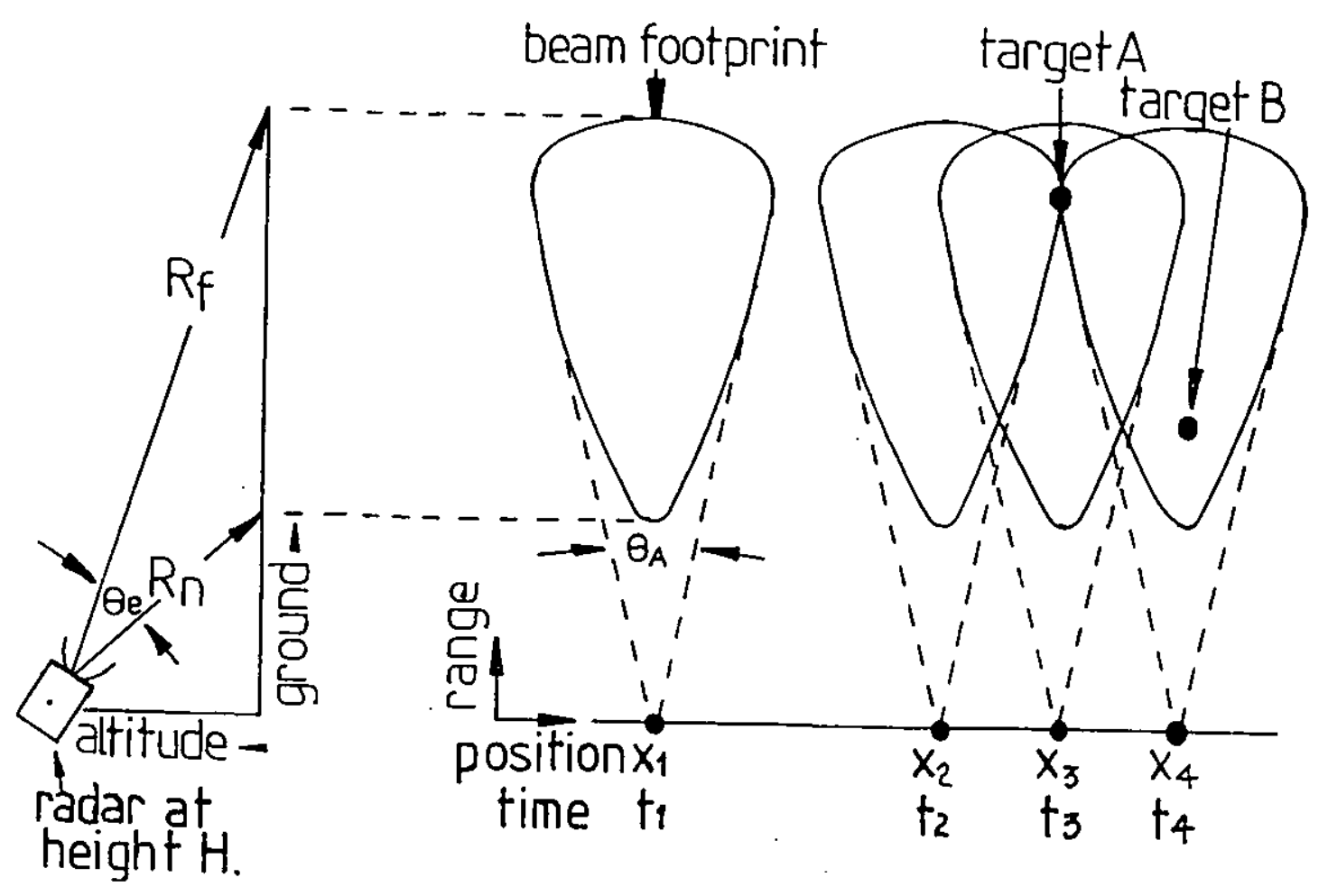

Fig.3.1(a) Elevation View.

Fig. 3.1(b) Plan View.

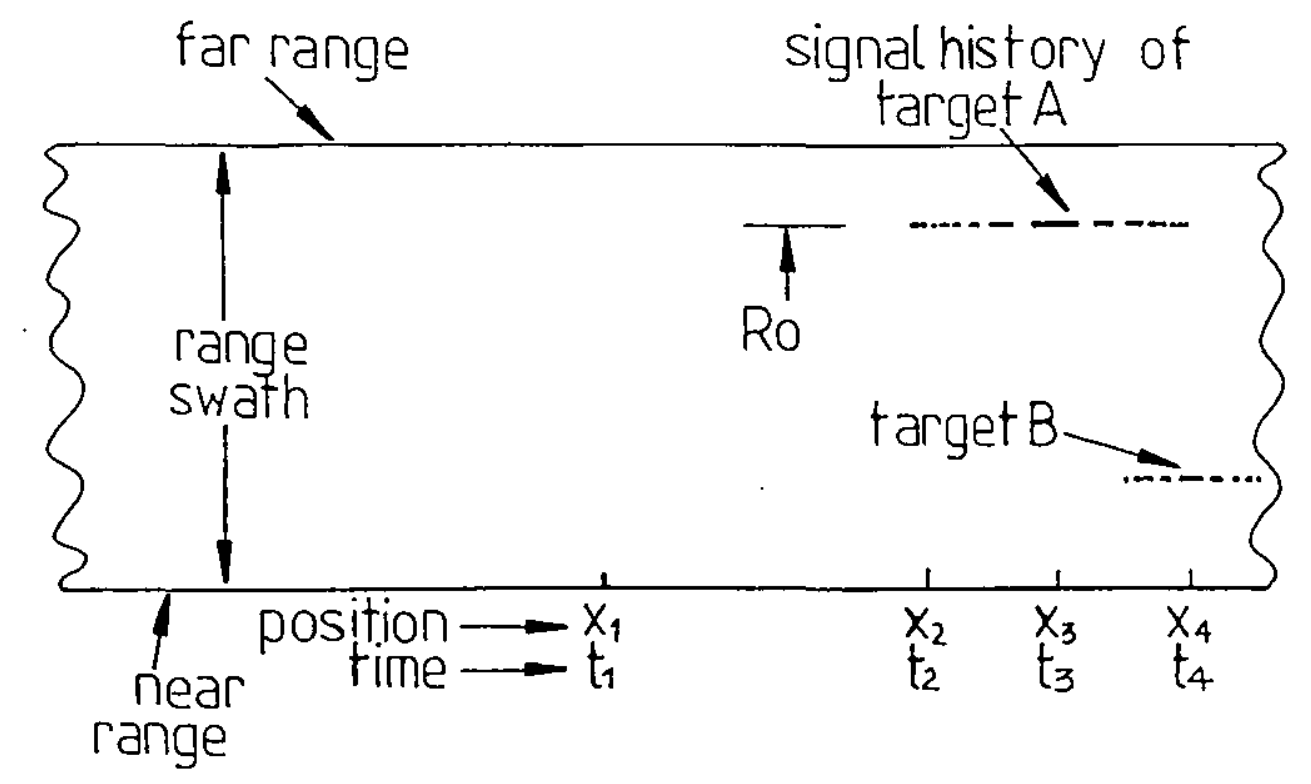

Fig. 3.1(c) Signal Film.

Fig. 3.1 Optical Processing of S.A.R. Data. 
near range $R_{n}$ and far range $R_{f}$. In $F i g$ 3.1(b) the plan view of the geometry is shown with the abscissa axis deplating the ground track position of the radar platform. which moves at a constant velocity. Thus, the abscissa axis can be considered as depicting either position $(x)$ or $t$ ime ( $t$ ). The ordinate axis is ground range and targets $A$ and $B$ are shown at different ranges. The radar antenna beam axis is orientated at right angles to the radar platform velocity, and the azimuth beam width is $\theta a$.

At time $t_{1}$. the radar is located at ground position $x_{1}$. the beam is at some distance from the first target $A$, so no signal is received. At a later $t_{i m e} t_{2}$ the radar is at position $\times_{2}$. Now the radar beam llluminates the target and a reflected signal is detected. In Fig. 3.1(c). a portion of the signal $f i l m$ strip is shown. The width of the signal film corresponds to the ground swathe illuminated by the radar beam. The received signal from each transmitted pulse is recorded across the width of film. Between pulses the film is advanced by small increments.

In Fig. $3.1(b)$, a target is first illuminated when the radar platform travels to position $x_{2}$, and in fig. $3.1(c)$ a target response is deplcted at range $R_{a}$. Thereafter, until time $t_{4}$ (or radar platform position $x_{4}$ ). eoch transmitted pulse will produce a detected signal. illustrated in Fig. 3.1(c). The target is illuminated between $t_{i m e} t_{2}$ and $t_{4}$. and this interval is called the integration or dwell time. The distance traversed by the radar platform during this time is the synthetic aperture length, and this is exactly the I inear beamwidth of the radar antenna at range $R_{2}$ [ [4] i.e. $x_{4}-x_{2}=\theta a R_{3}$. A second target $B$ is located at a range nearer than $R_{\text {. }}$. and the slgnal history length is 
much shorter as depicted in Fig. 3.1(c). The trace due to a target follows a hyperbolic arc as the distance between the target and the radar varies, but the very large distances involved in radar systems makes the trace appear as a straight line.

Referring to fig. 3.2 it will be noted that, as a target passes through the radar beam (due to radar platform motion), the slant range varies causing the relative phase of the reflected signal from a coherent transmitter to change from pulse to pulse. The rate of change of phase with time gives the Doppler frequency resulting from the relative motion between radar and target.

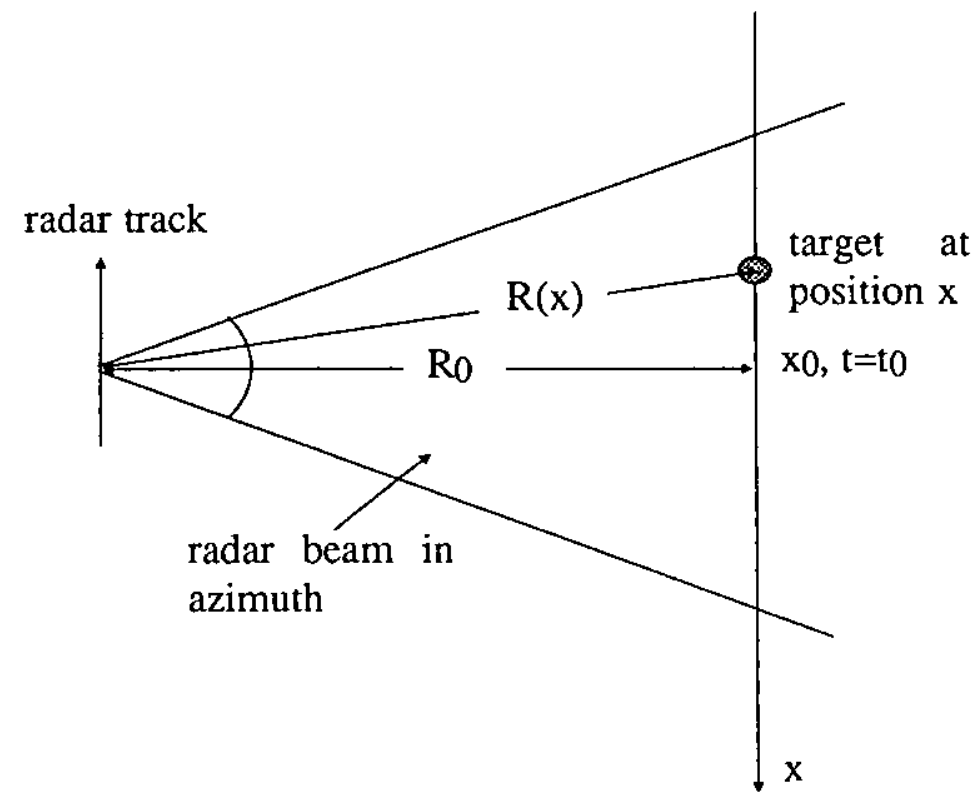

Fig. 3.2 Doppler Frequency of a Stationary Target.

In order to derive a simple expression for the Doppler frequency history of a stationary target passing 
through the beam, the curvature of the earth's surface and the earth's motion shall be ignored. Assume that at $t$ ime $t=t_{\text {. }}$. the target is at position $x=x_{0}$. where the $x$-axis represents the direction of motion of the target relative to the radar platform. This position marks the centre of the beam at which point the target and radar platform are at their minimum separation $\left(=R_{0}\right)$. From the geometry of Fig. 3.2, when the target is at some arbitrary position $x$ with respect to the radar platform. then the separation $R(x)$ is given by

$$
R(x)=\sqrt{ }\left[R_{0}^{2}+\left(x-x_{0}\right)^{2}\right]
$$

Since $x-x_{0}=v\left(t-t_{*}\right)$ and also $R_{0} \gg x_{*}$. then the separation can be expressed as a function of time. i.e.

$$
R(t)=R_{0}+\frac{\left(\nu\left(t-t_{a}\right)\right)^{2}}{2 R_{a}}
$$

The phase $\Phi(t)$ of the received signal from a point target can be derived from the distance between the radar antenna and the target and is given by

$$
\Phi(t)=\frac{4 \pi R(t)}{\lambda}
$$

(assuming that the phase is changing slowly)

The rate of change of $\Phi(t)$ with time gives the Doppler frequency $\omega d(t)$ :

$\omega d(t)=\frac{d \Phi(t)}{d t}=\frac{4 \pi v^{2}\left(t-t_{0}\right)}{\lambda R_{0}} \mathrm{rad} / \mathrm{s}$

Each target, therefore, produces a linear chirp 
centred on zero frequency, in the cross-range or azimuth dimension. sampled at the pulse rate. If the beam is not pointing in a direction perpendicular to its motion, but at a small squint angle to the perpendicular. then the result is a Doppler chirp with the same bandwidth and Doppler slope, but with a nonzero centre frequency. The signal amplitude and phase is recorded on $f i l m$ after phase coherent addition of the received signal with a reference signal. When the two signals are in phase, a higher level is obtained than when they are out of phase. When recorded on a film with the use of a narrow beam of 1 ight there will be a series of bright spots that are al igned parallel to the film direction with the spots closer together at the two ends. When a suitable Fresnel zone plate or lens is al igned over the series of spots. and a parallel beam of coherent light is passed through the lens and the signal film. the light beam is brought to a focus. This process is the equivalent to the mathematical fourier Transformation. Any misal ignments in the lens position will degrade the image.

To focus the signal history of a target at any range the lens must meet certain requirements. For a given azimuth resolution $\partial x$, the lens width corresponds to the synthetic aperture length and hence it is proportional to target range. To focus at every range the lens must be conical in shape. Figure 3.3 shows such a lens in on optical signal processor. A laser is used to provide a source of coherent light. which is used to Illuminate the signal film. The light then passes through the conical lens and is focussed on to the image film by another lens system. The signal $\mathrm{film}$ is then shifted by one azimuth resolution distance to produce the next line of images in the range direction. This process is repeated unt il the whole of the target has been reconstructed. The optical 
method of processing synthetic aperture data has been applied to terrain mapping radar $[5,8,9,29.30]$ and ultrasonic medical imaging $[10,31]$.

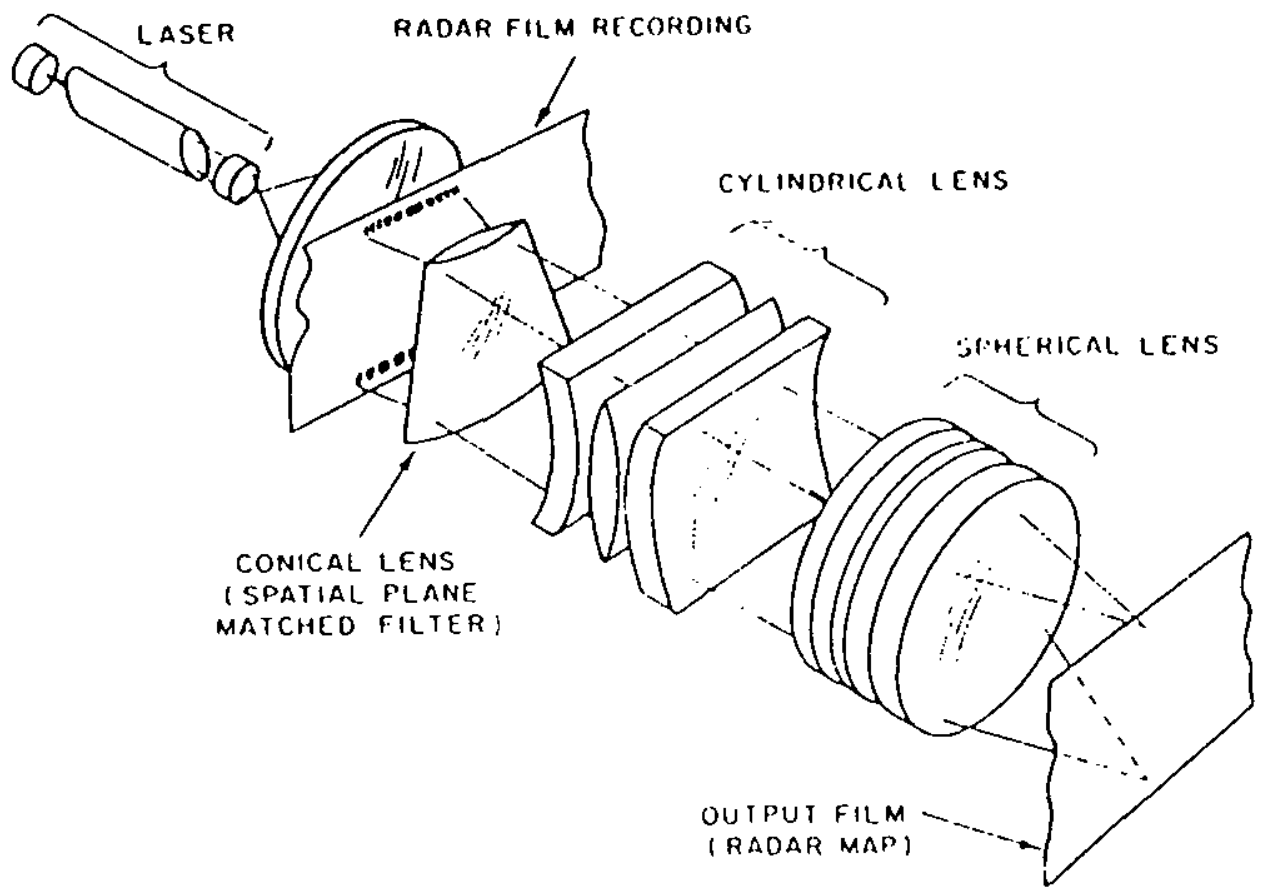

\section{Fig. 3.3 Optical Signal Processor.}

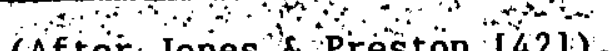

(After Jones \& Prestọn [42])

\section{3 Digital Signal Processing}

Digital signal processing offers the potential for more generalized processing operations and provide large dynamic range and can correct system aberrations and other image degrading effects [42,15,16,17] and have been used in sonar systems for many decades. 
Currently a wide spectrum of large scale integration devices (LSI), for example, high speed arithmetic processors and single chip microcomputers, provide sufficient processing power for complex real-time adaptive systems to be built.

Digital implementations utilize the advantages inherent in digital electronics: rellability. low cost. adjustment free operation and ready availability of a wide spectrum of complex circuit elements. In addition. since synthetic aperture systems only have a single channel (in most cases), a number of signal processing techniques, such as matched filter. can be considered, which would be difficult if the electronics had to be duplicated for every element channel of a large array.

Digital signal processing techniques in the frequency domain and the time domain will be discussed separately.

\section{3. 1 Signal Processing in the Frequency Domain}

Consider a point source at infinity. The amplitude and phase information of the received signal is stored for each position in the synthetic aperture. Fig. 3.4 shows the wavefront arriving at the aperture due to the point source.

The frequency of the sinusoldal variation across the aperture is proportional to the angle of incidence, $\theta$. of the arriving wavefront. An increasing angle $\theta$ gives an increasing frequency. When the object is directly in front of the aperture, $\theta=\theta$ and the signals at each position in the aperture is the same. Fourler transformation of the data values obtained across the aperture will produce a non-zero output in one of the aperture positions. 

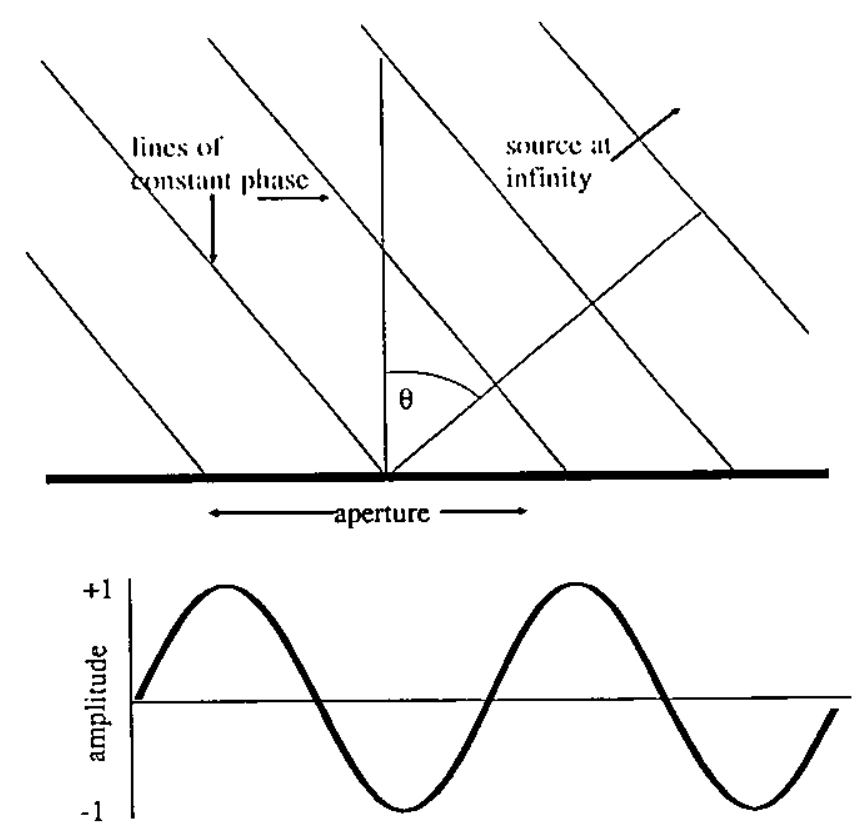

Fig. 3.4. Instantaneous Waveform along an Aperture due to a Sourceat Infinity

indicating the position of the source. The maximum resolvable frequency appears at the extremities of the aperture. The linearity properties of the fourier transformation allows complex images, which can be considered as a multiplicity of single points, to be reconstructed.

The of orementioned reconstruction is satisfactory when the target is situated at an infinite distance from the aperture. However, the majority of synthetic aperture systems operate within the near-field. Fig. 3.5 shows the wavefront resulting from a source, $T$, in the near-field. Here a Fresnel type distribution occurs. Sampling across the aperture now produces the variation shown, having additional frequency components. Fourier transformation 


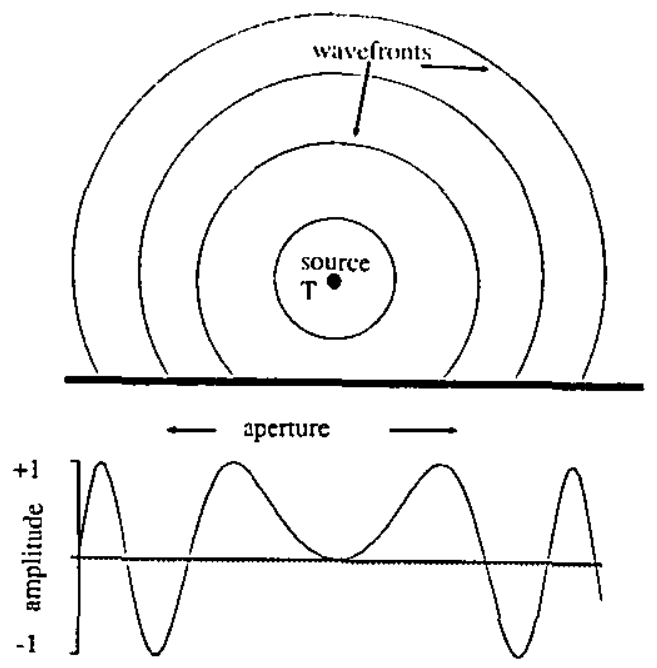

Fig. 3.5 Instantaneous Waveform along the Aperture due to a Source in the Near-field

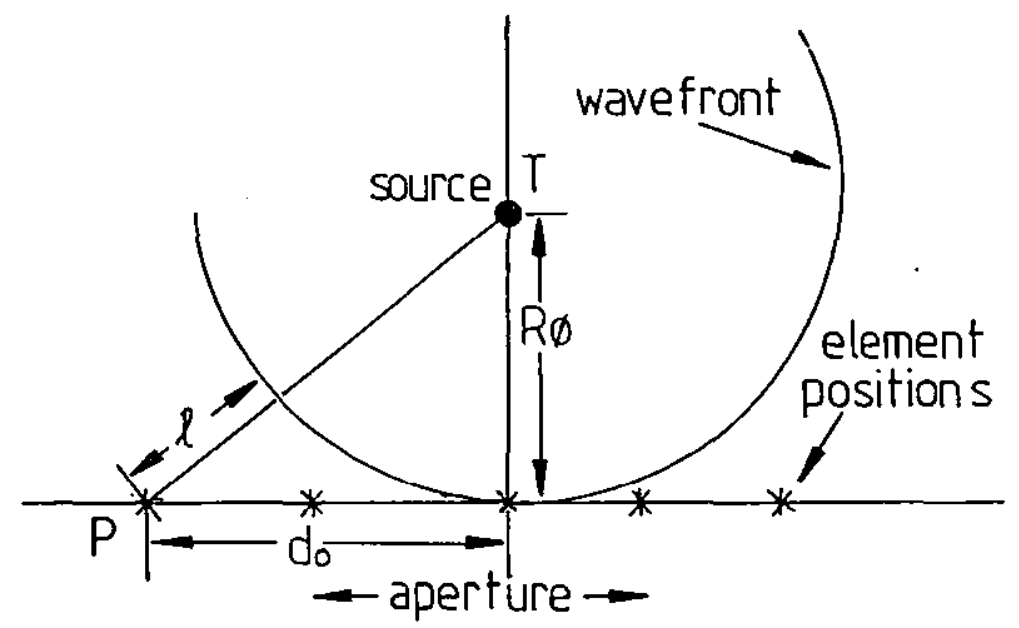

Fig. 3.6 Focussing Geometry 
now produces a multiplicity of spectral lines resulting in - defocused image.

Focussing effectively moves the source back to infinity and can be considered to be straightening the circular wavefronts, which are due to the differing paths lengths from the target to each aperture position. Fig. 3. 6 shows the path length, from a source. T. for one aperture position. $P$, at a distance, $d_{\text {. }}$. from the centre of the aperture. An increase in path length, $l$, produces a phase lag given by $\exp (\mathrm{j} 2 \pi \lambda / \lambda)$ for a given source ( or target) to element position distance, calculation of the extra path length, allows the corresponding phase lags to be calculated. Multiplication of the recelved signal by a phase lead $\exp (j 2 \pi l / \lambda)$ effectively straightens the curved wavefront. A single spectral component is produced again on transformation.

Systems using frequency processing have been developed [34.36] and Nagai [35] has proposed a method of reconstruction that reduces the computation $t$ ime from $N^{3}$. in conventional frequency domain systems, to $8 N^{2} \log _{2} N$, where $N$ is the number of Image pixels in one direction.

\section{3.2 Signal Processing in the Time Domain}

In time domain signal processing, each imoge pixel is reconstructed by the summation of the signals received along the aperture. having first been properly delayed to compensate for the acoustic path differences between the target and the aperture positions. This delay and summing is achieved in different ways, elther by hardware analogue multiplication [37] or by software implementation of algorithms [32]. The latter method will be discussed in 
detail. describing the technique of image reconstruction for synthetic aperture systems.

Consider the geometry of Fig. 3.7. showing a transducer moving in a straight line parallel to the $x$-axis. The transducer is located at position $P$. $\left(x_{a}, y_{x}\right)$, and a single point target. $T$, is located at $\left(x_{1}, y_{1}\right)$. The transmitted signal $S(t)$, of pulse length $T_{\text {s }}$ is given by

$$
S(t)=A \sin (2 \pi f, t)
$$

The reflected signal from the point target. T. is given by

$$
R\left(x_{0} \cdot t\right)=A \sin \left(2 \pi f_{0}(t-\tau)\right)
$$
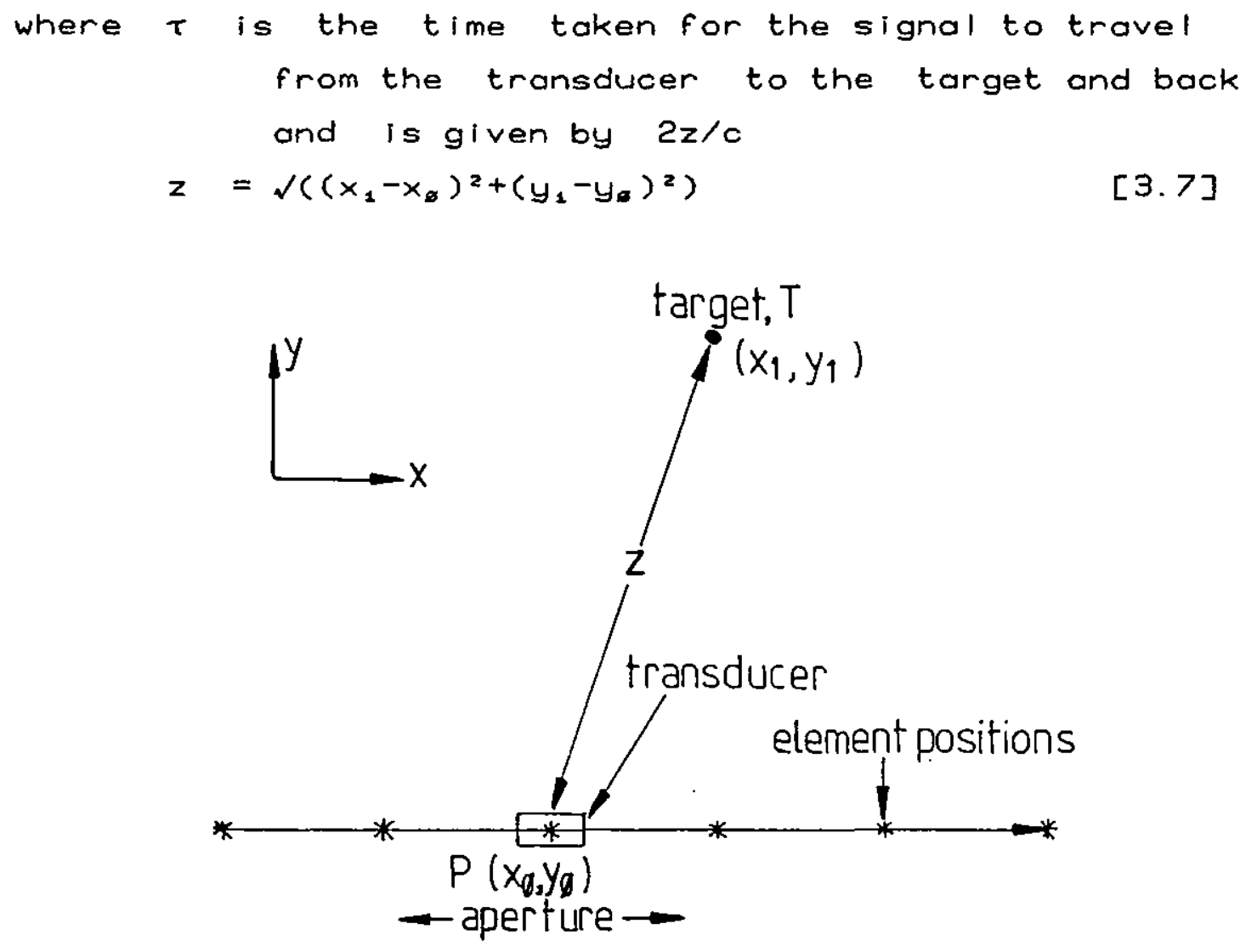

Fig 3.7 System Geometry 
It is assumed that there is no attenuation due to absorption, scattering or transducer inefficiency. Demodulation of the received signal yields both quadrature components (detailed in section 3.2.3).

$$
\begin{aligned}
& U_{i}\left(x_{0}, t\right)=R\left(x_{0}, t\right) \cos \left(2 \pi f_{\Delta} t\right) \\
& =\frac{A}{2}\left[\sin \left(\omega_{*}(2 t-\tau)\right)-\sin \left(\omega_{0} \tau\right)\right] \\
& U_{q}\left(x_{g}, t\right)=R\left(x_{s}, t\right) \sin (2 \pi f, t) \\
& =\underline{A}\left[\cos \left(\omega_{0}(2 t-\tau)\right)+\cos \left(\omega_{\gamma} \tau\right)\right] \\
& 2
\end{aligned}
$$

Removing the sum frequency component by low pass filtering gives

$$
\begin{aligned}
& v_{i}\left(x_{0}, t\right)=\frac{-A}{2} \sin \left(\omega_{0} \tau\right) \\
& v_{q}\left(x_{0}, t\right)=\frac{A \cos \left(\omega_{0} \tau\right)}{2}
\end{aligned}
$$

Figure 3.8 shows the waveforms of the various signals. The time variation, $t$, is proportional to the distance of the spherical wavefront. $E$. Thus substituting

$$
t=\frac{2 \xi}{c}, \quad \tau=\frac{2 z}{c} \text {, and } \lambda=\frac{c}{f_{c}}
$$

into equations 3.12 and 3.13 gives

$$
\begin{aligned}
& v_{1}\left(x_{0}, \xi_{0}\right)=-\frac{A}{2} \sin \frac{(4 \pi z)}{\lambda} \\
& v_{q}\left(x_{0} . \xi_{0}\right)=\frac{A}{2} \cos \frac{(4 \pi z)}{\lambda}
\end{aligned}
$$

$v_{i}$ and $v_{q}$ represent the response to a point target a distance $z$ from the transducer. Figure 3.9 shows the variation of $v_{i}$ and $v_{q}$ across the aperture.

If the transducer is moved along the aperture and the demodulated and filtered echoes recorded, the result is a pair of two dimensional arrays with echoes from a 


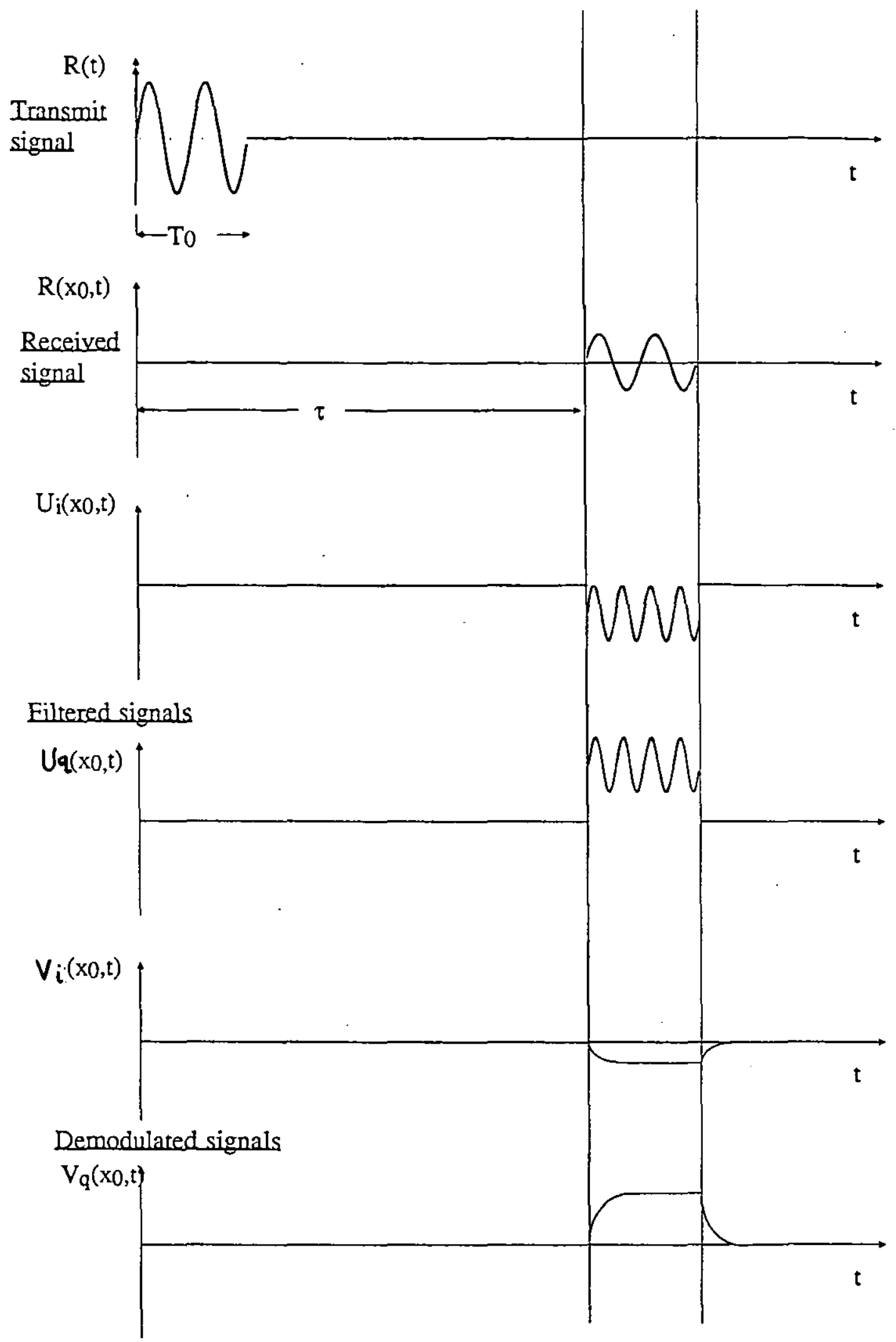

Fig, 3.8 Typical Received Waveforms for a Synthetic Aperture System using Baseband Components. 

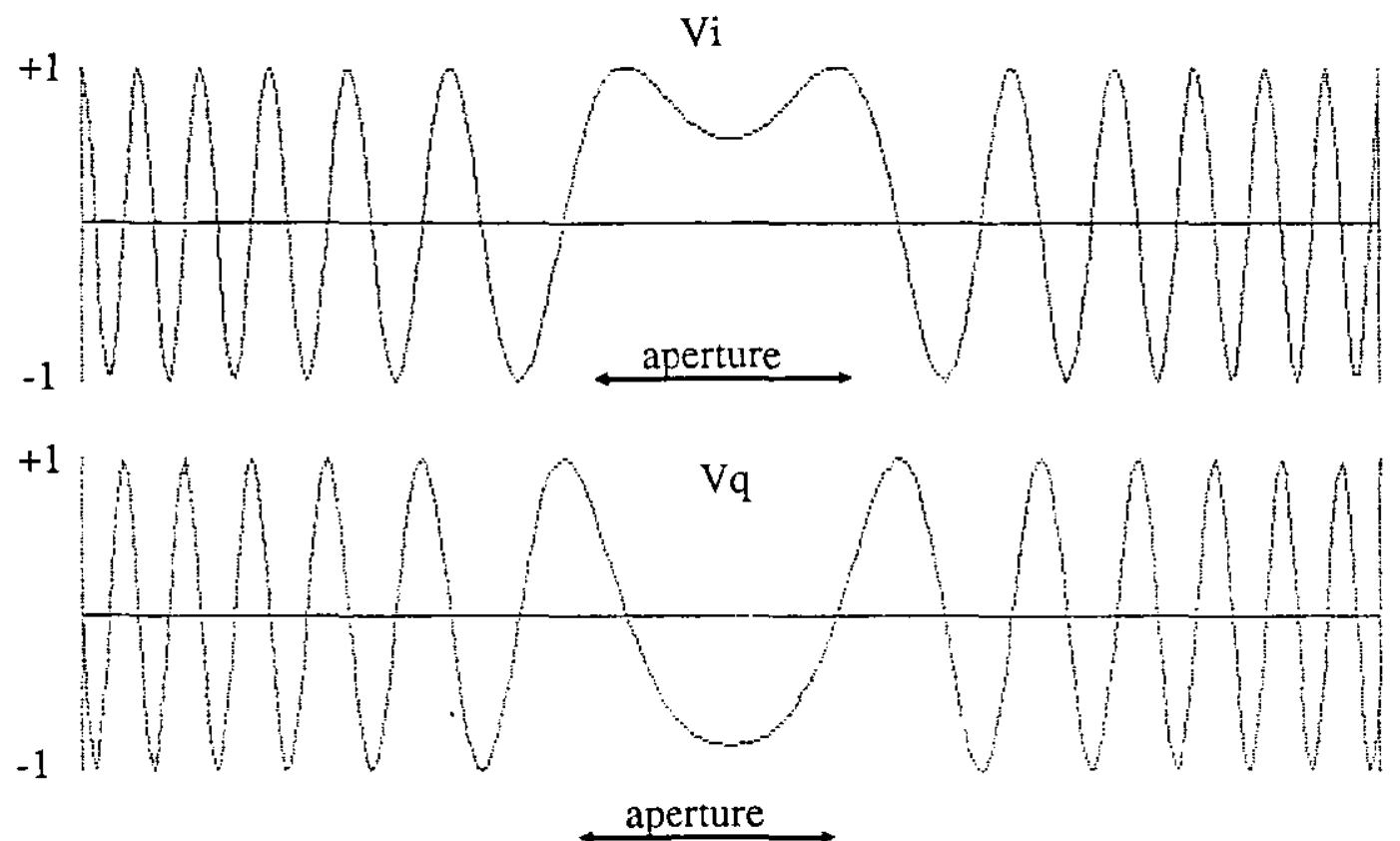

Fig. 3.9 Variation of $\mathrm{Vi}$ and $\mathrm{Vq}$ across an Aperture of $1 \mathrm{~m}$ due to Single Target at $4 \mathrm{~m}$, when the Frequency is $150 \mathrm{kHz}$. 
target lying on a hyperbolic arc. The shape of the hyperbolic arc is dependent upon the minimum distance between the target and the transducer. Once the transducer has moved to the end of the aperture, image reconstruction can commence. The reconstruction process can be described as a matched filter using equations 3.13 and 3.12 as the impulse response.

Each image point $G\left(x_{2}, y_{2}\right)$, is formed by integrating the data, due to a target at that position. in the data arrays. The data due to a point target lies in a hyperbolic arc, and hence the integration path must 1 ie along the same hyperbolic path. Thus

$$
G\left(x_{2} \cdot y_{2}\right)=\int_{0}^{x_{\max }} \int_{0}^{\varepsilon_{\max }} v_{q} \cos \frac{\left(4 \pi z_{2}(x)\right)}{\lambda}-v_{i} \sin \frac{\left(4 \pi z_{2}(x)\right)}{\lambda} d x d \xi[3.16]
$$

where $z_{2}(x)=\sqrt{ }\left(\left(x_{2}-x\right)^{2}+\left(y_{2}-y\right)^{2}\right)$

The double integral describes the hyperbolic path that constitutes the integral path and the summation of the data received at each position in the synthetic aperture. The geometry of the reconstruction process is shown in Figure 3.10. An image is obtained when every image point has been reconstructed.

\subsubsection{Baseband Representation of Band Limited Signals}

Signals of the type found in sonar applications are, in many cases, band limited. A naive application of the Nyquist criterion would indicate a sampling rate much higher than that actually required. Consider Fig. 3.11. showing the spectrum of a typical ultrasonic signal. with a 


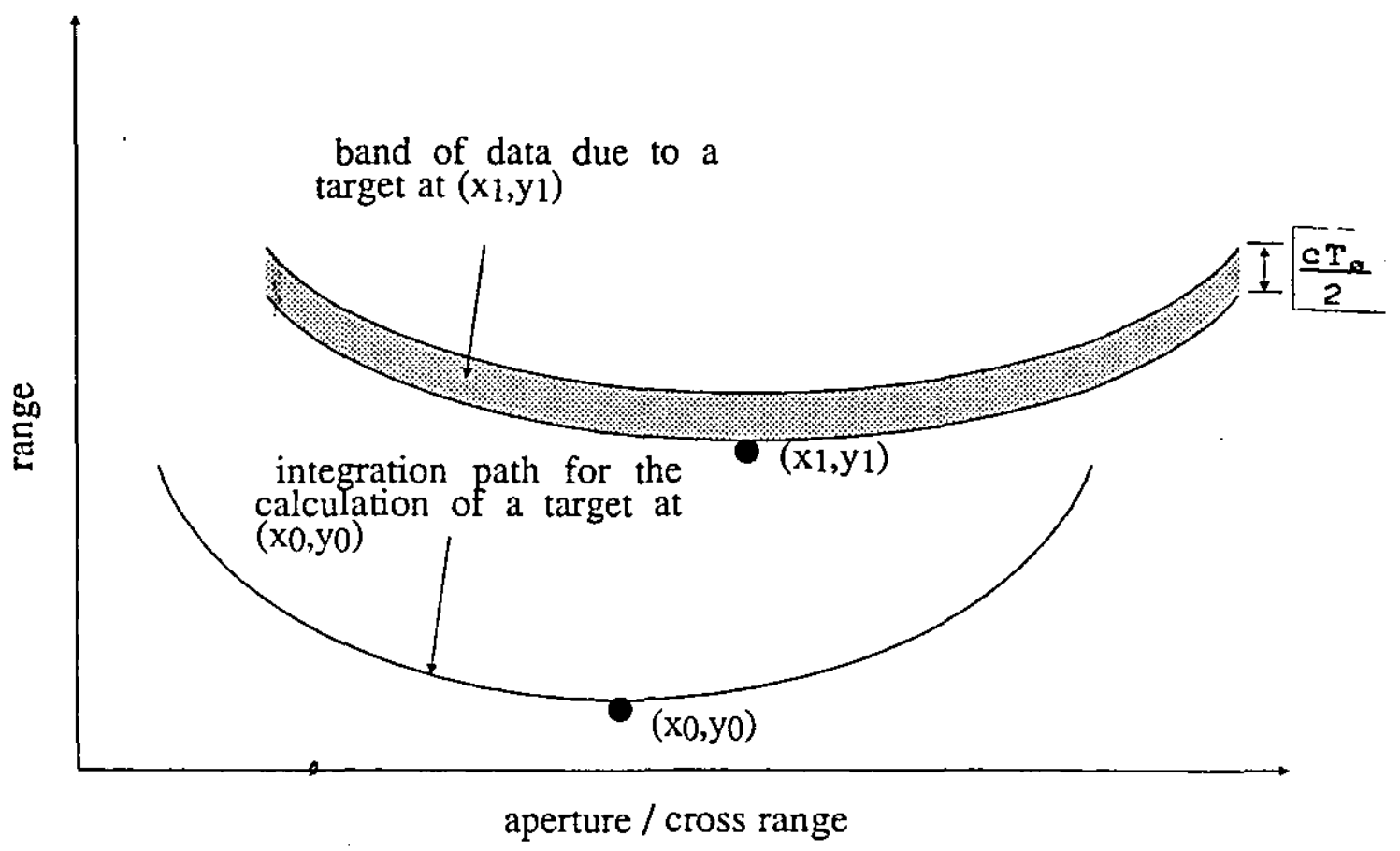

Fig, 3.10 The Integration Path used in the Image Reconstruction Process

range
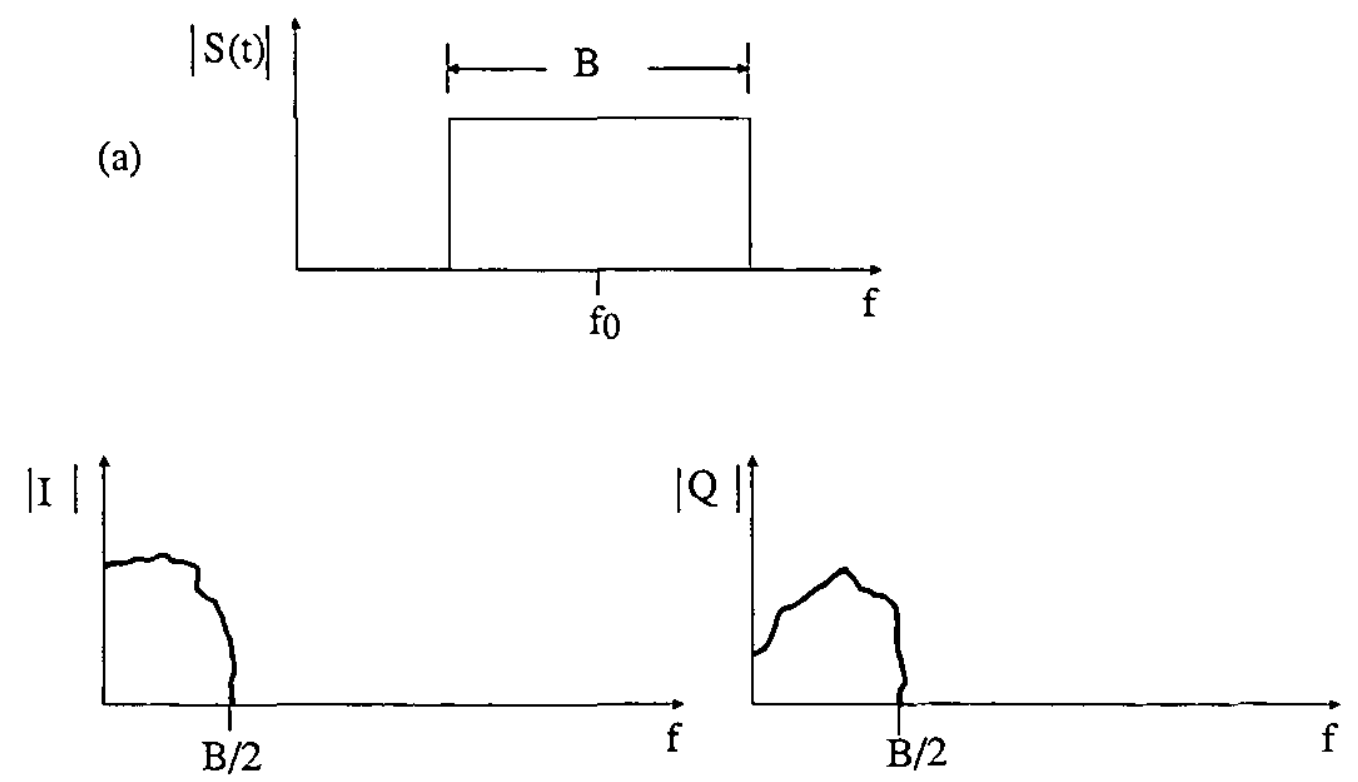

(b)

Fig. 3.11 Reducing the Sampling Rate by using Baseband Quadrature Signals; (a) Spectrum of Received Signal, (b) Spectrum of the Baseband Signals. 
centre frequency of $f$ and a bandwidth $B$.

The minimum sampling frequency using the Nyquist criterion is $f s$, where

$$
f s=2\left(f_{\sigma}+\frac{B}{2}\right)=2 f_{\sigma}+B
$$

If this signal was translated to baseband, however, two quadrature channels would be required, but the sampling rate on each one would only be $f s^{\prime}$. where

$$
f s^{\circ}=2\left(\frac{B}{2}\right)=B
$$

This is a sampling rate reduction of $n r$. where

$$
\eta r=\frac{f s^{\circ}}{f s}=\frac{\gamma}{(2+\gamma)}
$$

and $r$ is the relative bandwidth and is equal to $B / f$.

In ultrasound systems, for example, $r=0.1$ and $n r=$ 0.05. a significant reduction. Fig. 3.12 illustrates how the quadrature components $I(t)$ and $Q(t)$ can be obtained from a received signal.

This sampling rate reduction also has an impact on the data storage requirements of synthetic aperture system.

The amount of digital data storage required is the product of the data acquisition time and the sampling rate. Hence the data storage reduction factor using baseband signals is na. where

$$
n q=\frac{2 f s^{\cdot} T}{f s T}=2 n r=\frac{2 \gamma}{(2+\gamma)}
$$


where $T$ is the $t$ ime length of the data sample, and the factor 2 reflects the requirement for the two quadrature channels. Using the typical case numbers from above. $n q=0.1$.

To image a maximum range $R$ with an array of $N$ trandsucers requires a data receive time of approximate length $2 R / c$, where $c$ is the acoustic velocity in the medium and assuming a single frequency system. Hence the total data storage is 0 . where

$$
Q=2 f s^{\prime} T N=\frac{4 B N R}{c}
$$

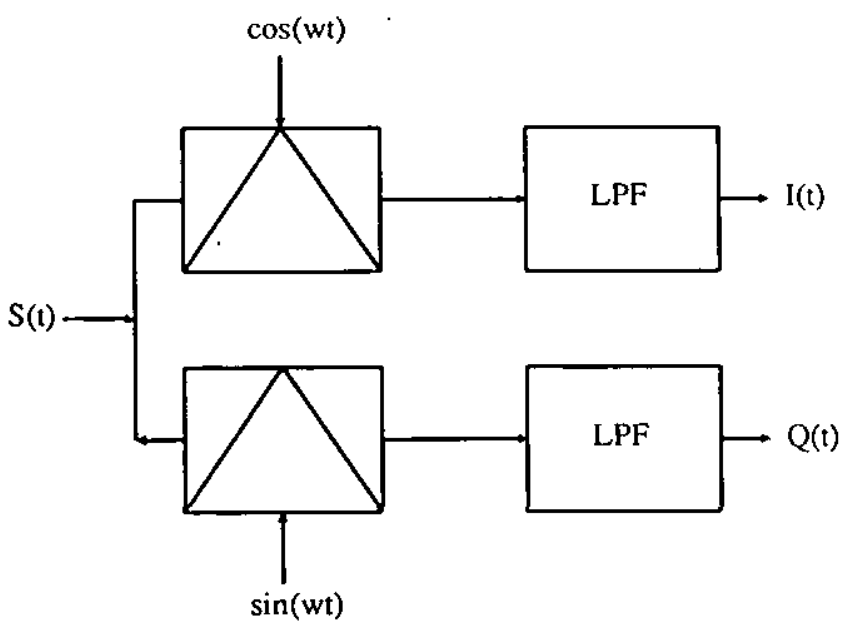

Eig. 3.12 Obtaining Quadrature_Baseband Signals from_a Band Limited Signal 


\section{SIMLLATION}

\subsection{INTRODUCTION}

In this chapter various target configurations will be simulated and discussed. Basic mathematical analysis is carried out and the expected data waveforms with the corresponding reconstructed images illustrated. The latter section deals with the problems coused by motion errors leading to uncertainty in transducer position. The system required to reduce these errors has been simulated and the results of motion error phase correction will be discussed.

\subsection{Sustem Simulation for a Single Target}

Consider the situation shown in Fig. 4.1. where the tranducer is at element position, $P\left(x_{0} \cdot y_{0}\right)$, and transmits a pulsed signal $S(t)$ where

$$
\begin{aligned}
& S(t)=A \cos (\omega t) \\
& \text { and } A \text { is the amplitude of the signal. }
\end{aligned}
$$$$
[4.1]
$$

The signal received by the tranducer due to a point target. T, $\left(x_{1}, y_{1}\right)$, is

$$
\begin{aligned}
& R(t)=\frac{A}{z 1^{2}} \cos \left(\omega t+\frac{4 \pi z 1}{\lambda}\right) \\
& \text { where } z 1=\sqrt{ }\left(\left(x_{1}-x_{0}\right)^{2}+\left(y_{1}-y_{0}\right)^{2}\right)
\end{aligned}
$$

Hence the amplitude of the received signal 


$$
|R(t)|=\frac{A}{z 1^{2}}
$$

and the phase

$$
\angle R(t)=\frac{4 \pi z 1}{\lambda}
$$

A plat of the amplitude and phase of the received signal along an aperture of $1 \mathrm{~m}$ for a point target at a range of $20 \mathrm{~m}$, and an element spacing of $\lambda / 2$ at a frequency of $150 \mathrm{KHz}$ is illustrated in Fig. 4.2. The reconstructed target is shown in Fig. 4.3.

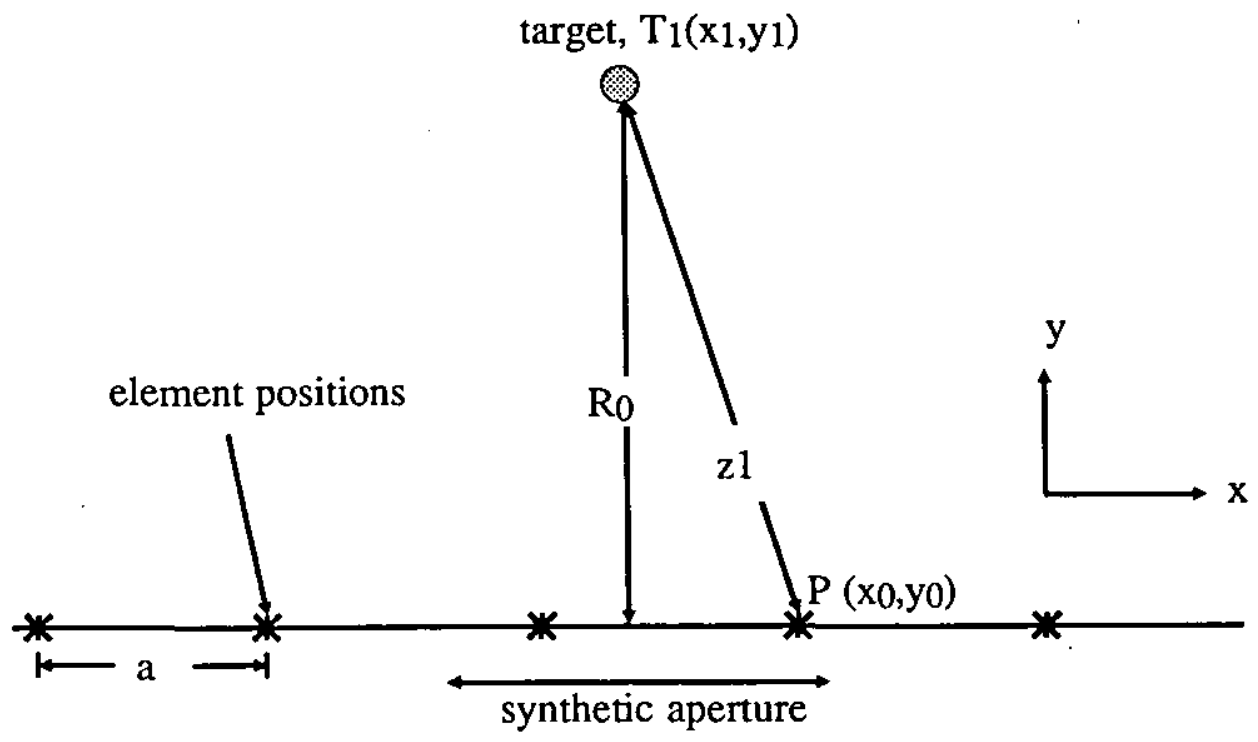

Fig. 4.1 Simulation of a Single Target.

\subsection{System Simulation for Two Torgets}

Now consider the case shown in Fig. 4.4 where there are two targets. T1 $\left(x_{1}, y_{1}\right)$ and $T 2\left(x_{2}, y_{2}\right)$, at a distance of $R_{\text {o }}$ from the aperture. 

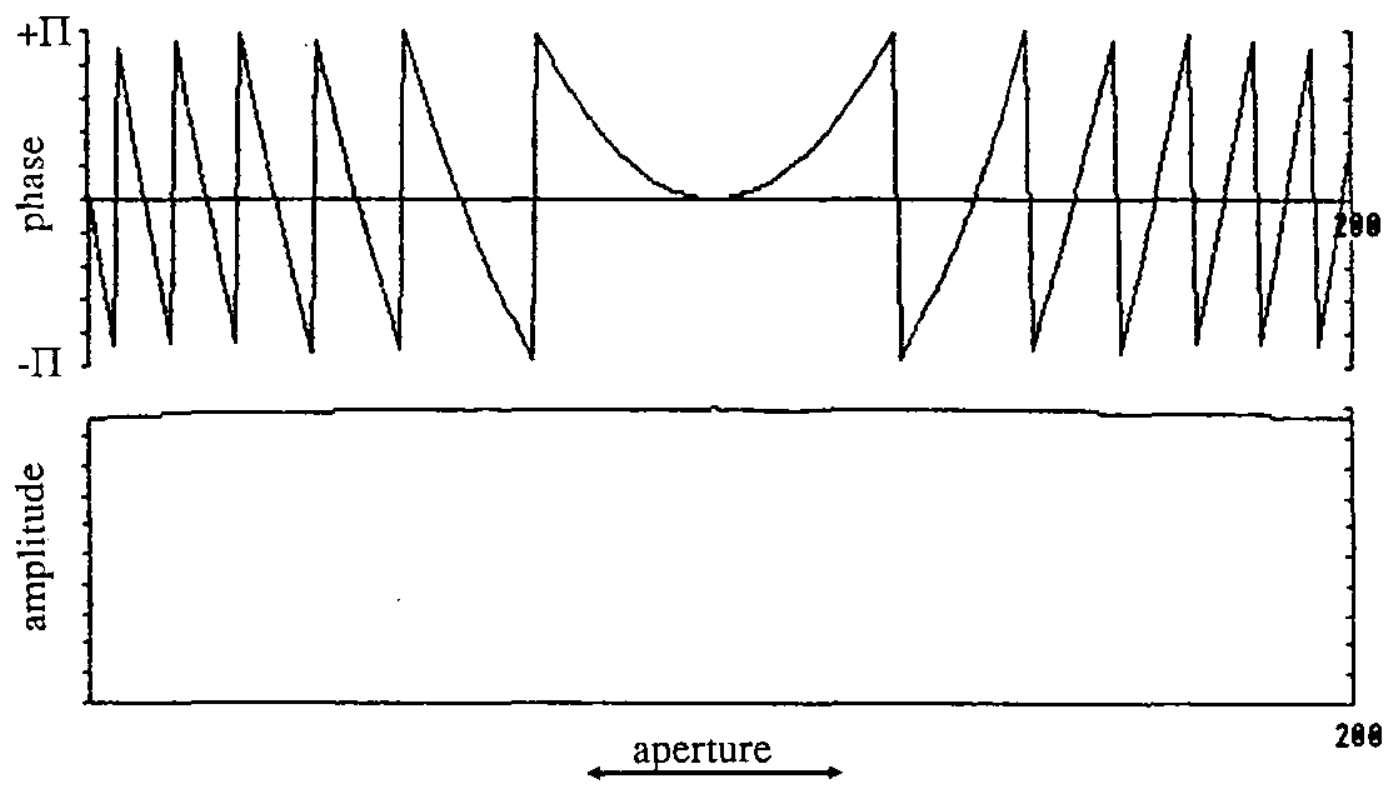

Fig, 4.2 Amplitude and Phase Variation across the Aperture due to a Single Target at a Range of $20 \mathrm{~m}$.

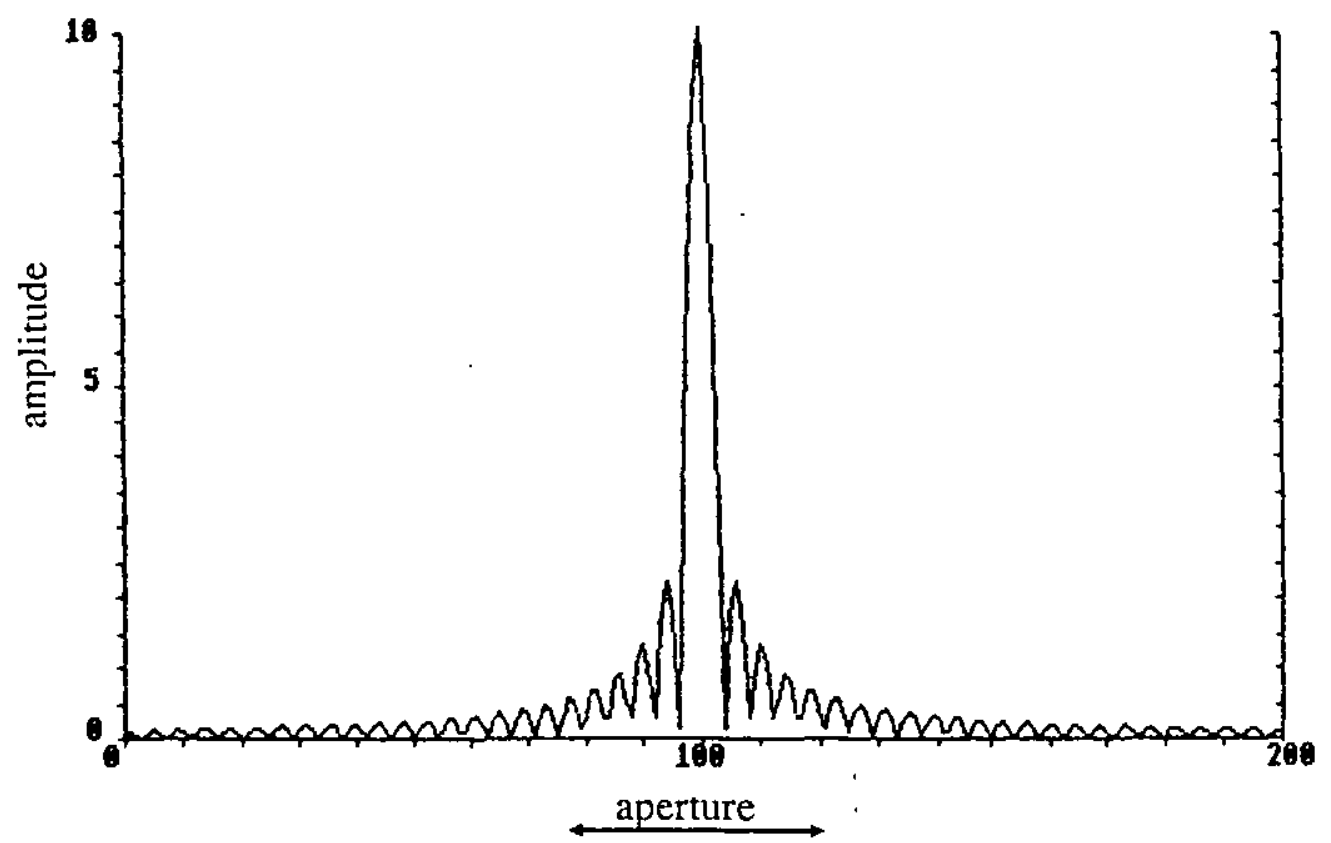

Fig. 4.3 Reconstructed Image of the Single Target. 
A pulsed signal $S(t)$ is transmitted from the transmitter at element position $P\left(x_{0}, y_{0}\right)$ and the received signal due to the two targets is

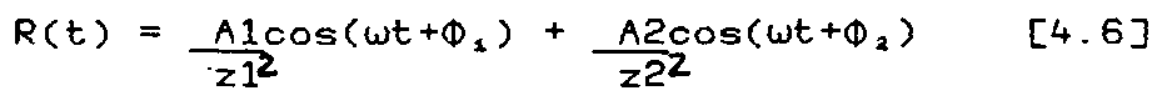

$$
\begin{aligned}
& \text { where } z 1=\sqrt{ }\left(\left(x_{1}-x_{0}\right)^{2}+\left(y_{1}-y_{0}\right)^{2}\right) \text { [4.7] } \\
& z 2=\sqrt{ }\left(\left(x_{2}-x_{0}\right)^{2}+\left(y_{2}-y_{0}\right)^{2}\right) \quad[4.8] \\
& \Phi_{1}=\frac{4 \pi z 1}{\lambda} \quad[4.9] \\
& \Phi_{2}=\frac{4 \pi z 2}{\lambda} \quad[4.10]
\end{aligned}
$$

and $A 1$ and $A 2$ reflect differences in target strengths.

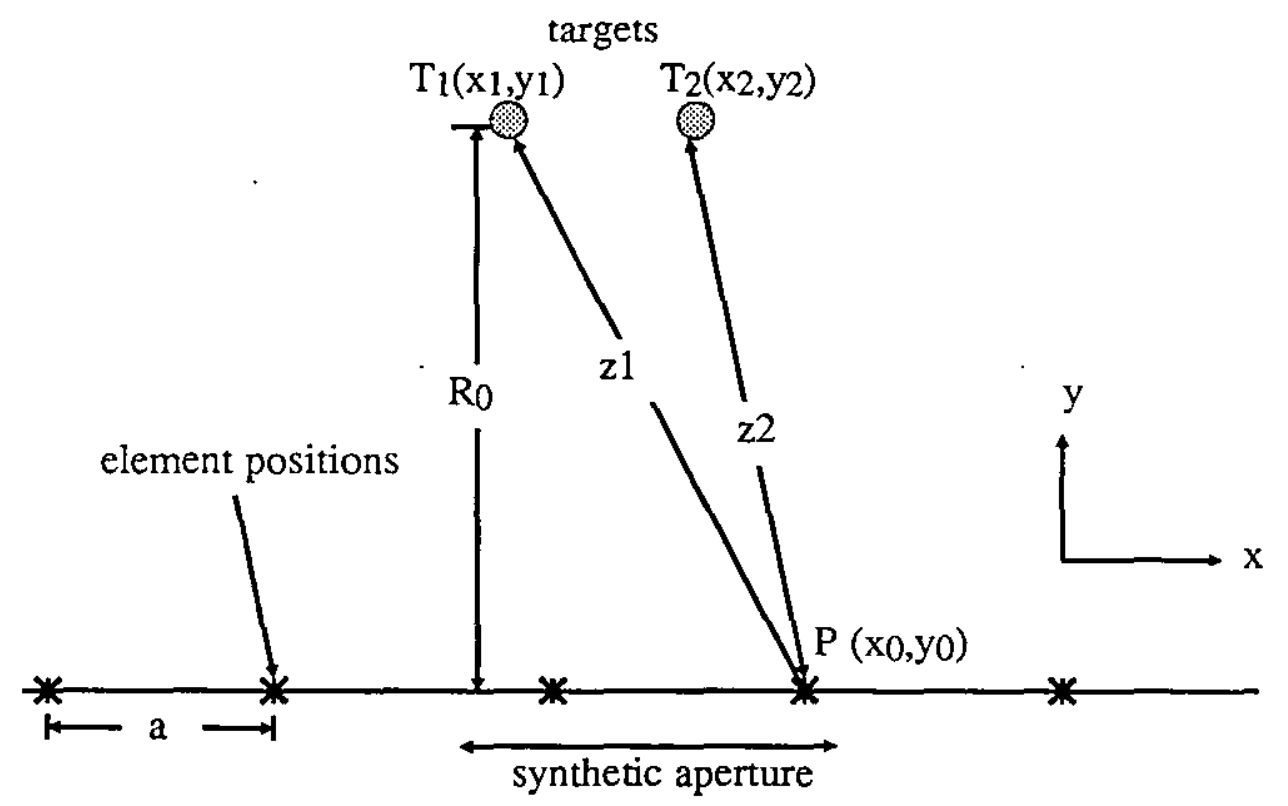

Fig. 4.4 Simulation of Two Similar Targets at the Same Range.

Hence

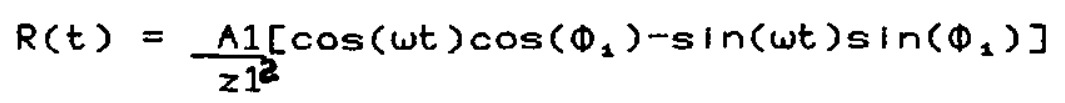




$$
\begin{aligned}
& +\frac{A 2}{z 2^{2}}\left[\cos (\omega t) \cos \left(\Phi_{2}\right)-\sin (\omega t) \sin \left(\Phi_{2}\right)\right] \quad[4.11] \\
& =\cos (\omega t)\left[\frac{A 1}{21^{2}} \cos \left(\Phi_{1}\right)+\frac{A 2}{22^{2}} \cos \left(\Phi_{2}\right)\right] \\
& -\sin (\omega t)\left[\frac{A 1}{21^{2}} \sin \left(\Phi_{1}\right)+\frac{A 2}{22^{2}} \sin \left(\Phi_{2}\right)\right][4.12]
\end{aligned}
$$

Thus the amplitude of the recelved signal

$$
|R(t)|=\sqrt{ }\left(\frac{A 1^{2}}{21^{4}}+\frac{A 2^{2}}{22^{4}}+\frac{A 1 A 2}{21^{2} 22^{2}} \cos \left(\Phi_{1}-\Phi_{2}\right)\right) \quad[4.13]
$$

and the phase

$$
\angle R(t)=\tan ^{-1} \frac{\left[\left(A_{1} / z_{1}^{2}\right) \sin \left(\Phi_{1}\right)+\left(A 2 / \mathrm{z}^{2}\right) \sin \left(\Phi_{2}\right)\right]}{\left(A 1 / z^{2}\right) \cos \left(\Phi_{1}\right)+\left(A 2 / \mathrm{z}^{2}\right) \cos \left(\Phi_{2}\right)}[4.14]
$$

Fig. 4.5 shows the variation of the recelved signal amplitude and phase along an aperture of $1 \mathrm{~m}$ from two similar targets at a range of $20 \mathrm{~m}$, with a separation of $0.5 \mathrm{~m}$. with an operating frequency of $150 \mathrm{kHz}$. The reconstructed targets are shown in F1g. 4.6

\subsection{Simulation of Motion Errors}

The successful application of the sunthetic aperture technique relies on the fact that coherency of the received signals must be malntalned for the whole length of the aperture. Unknown movements in the transducer as $1 t$ traverses the aperture will ieas to the loss of coherency of the recelved $s i g n a l$ and a degradation in the Image obtained $[4.5-50]$.

Two types of motion error, random and biased. will be simulated and techniques to reduce the degradation In the reconstructed image due to transducer motion exrors w111 be discussed. 

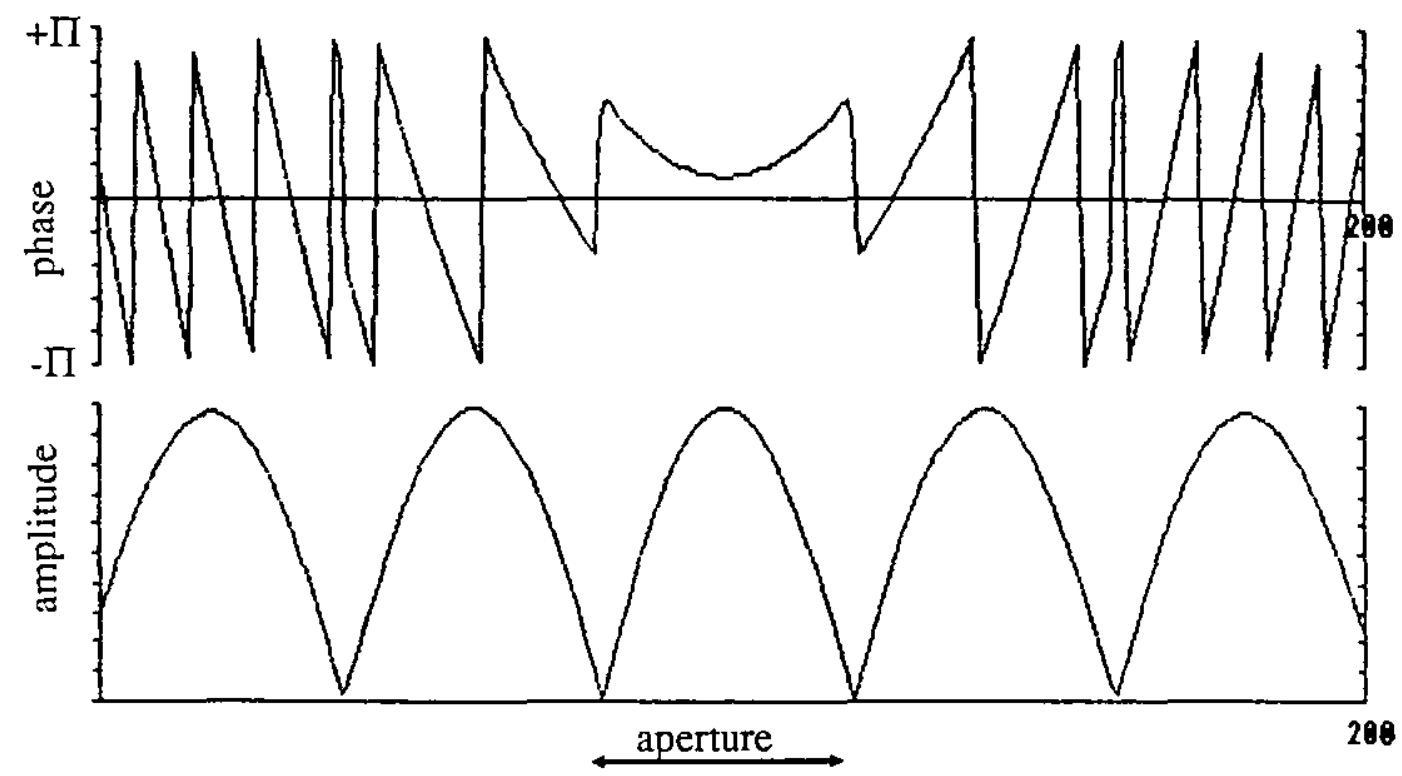

Fig, 4.5 Amplitude and Phase Variation across the Aperture due to a Pair of Targets at a Range of $20 \mathrm{~m}$.

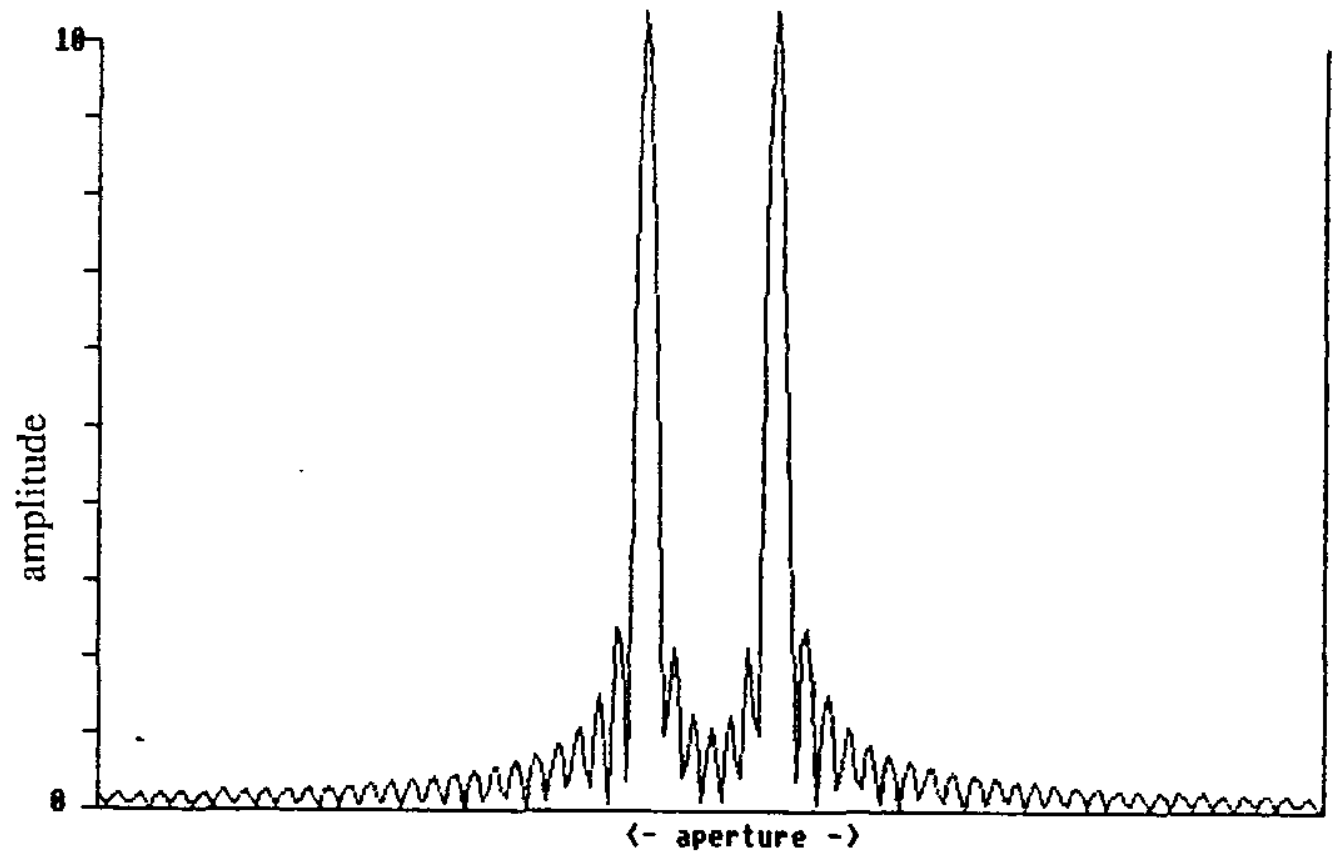

Eig, 4.6 Reconstructed Image of the Pair of Targets. 


\subsubsection{Errors due to Random Transducer Mot ion}

The effects of random transducer motion errors on the reconstruction of a single target is shown in fig. $4.7(a)$ to Fig. 4.7(d). The synthetic aperture is generated by moving the receiver tronsducer only and the transmitter is assumed to be stationary. An aperture length of $128 \lambda$ is generated at an operating frequency of 40 $\mathrm{kHz}$. The element spacing is $\lambda / 2$. Fig. $4.7(a)$ illustrates that the effects of random motion of the transducer. of amplitude $<0.1 \lambda$ are negligible. There is no noticable image degradation. With a random error amplitude of $0.5 \lambda$. Fig. $4.7(b)$ Illustrates that there is a reduction in the amplitude of the main lobe and an increase in the side-lobe level. Increasing the random motion error amplitude to $0.7 \lambda$ and $1 \lambda$ results in further reduction of the main beam amplitude, as illustrated in Fig. 4.7(c) and Fig. 4.7(d) respectively. The target is no longer reconstructed in Fig. 4.7(d). Increasing the amplitude of the random motion therefore. increases the generation of spurious targets and increases the level of the side-lobes. When the transducer random motion is equal to or greater than $1 \lambda$ the target is no longer distinguishable from the noise.

\subsubsection{Errors due to Biased Transducer Motion}

The two parameters that can be varied when the error motion is biased are "peak amplitude" of the biased error motion and the "frequency" of the biased error motion. Figure 4.8 illustrates both of these quantities. The amplitude of the biased error motion is the maximum distance (in wavelengths) between the actual transducer position and the expected transducer position (with no motion error). The frequency of the biased error motion is 


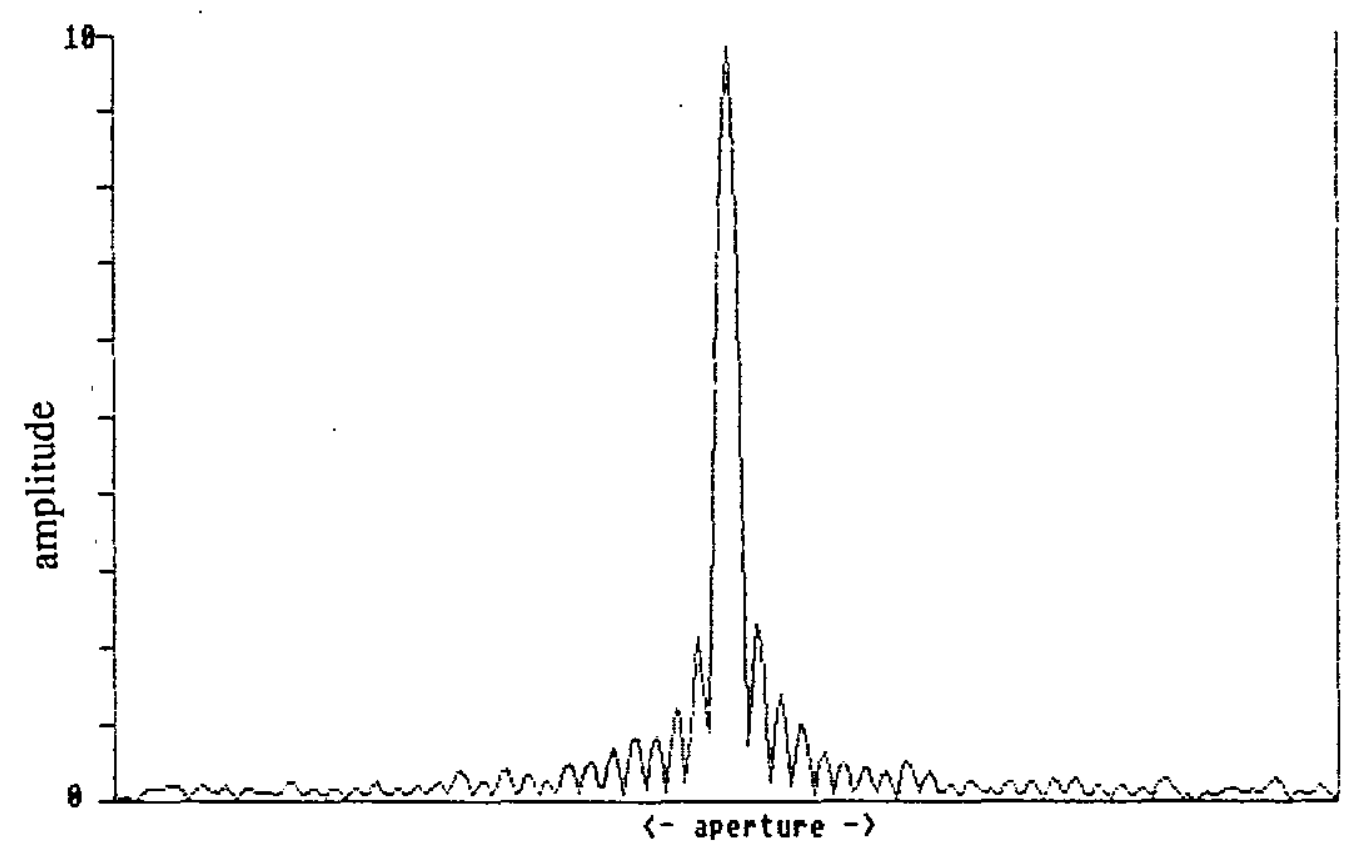

Fig, 4.7(a) Reconstructed Image with a Random Transducer Error Motion of $<0.1 \lambda$.

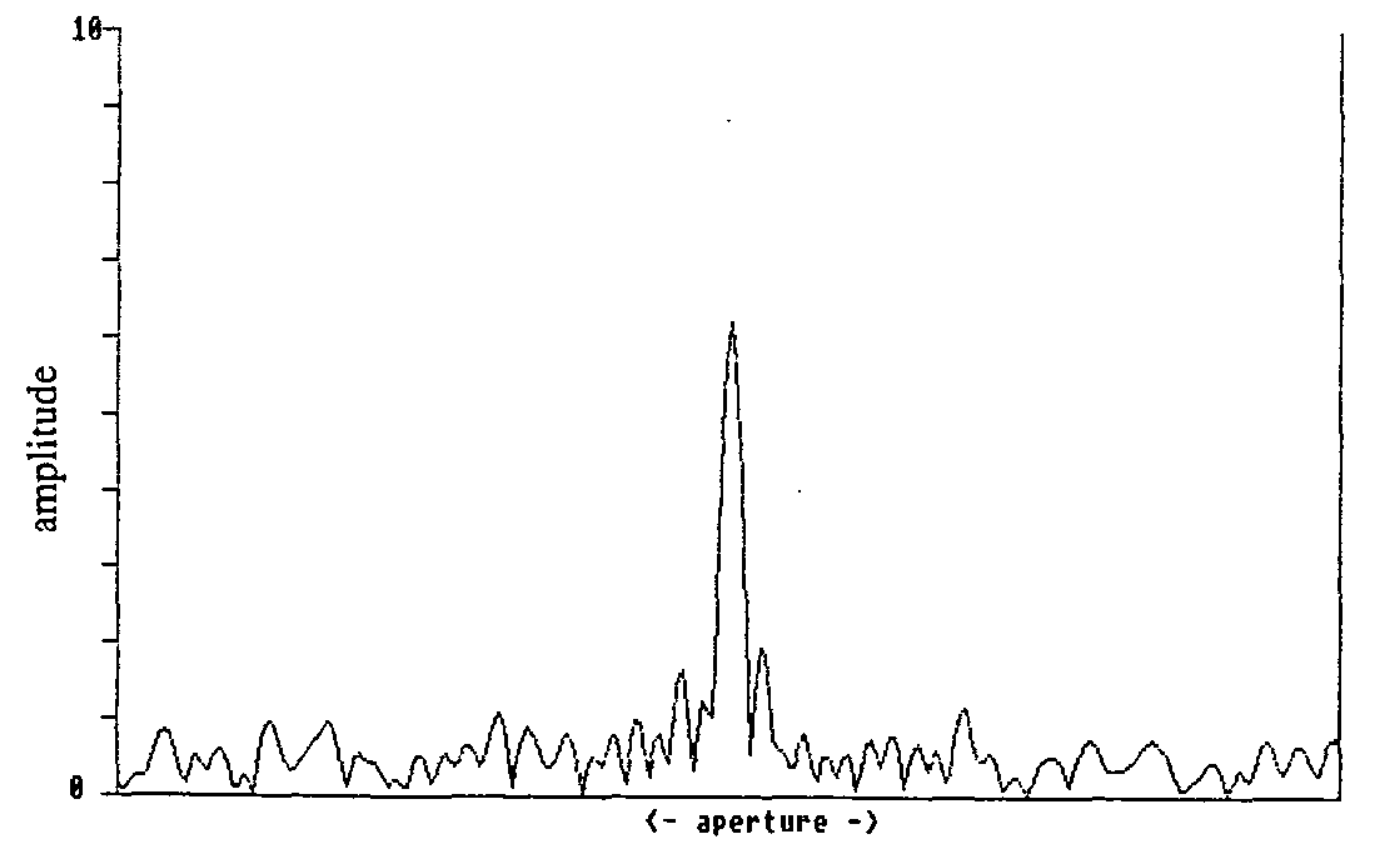

Fig. 4.7(b) Reconstructed Image with a Random Transducer Error Motion of $<0.5 \lambda$. 


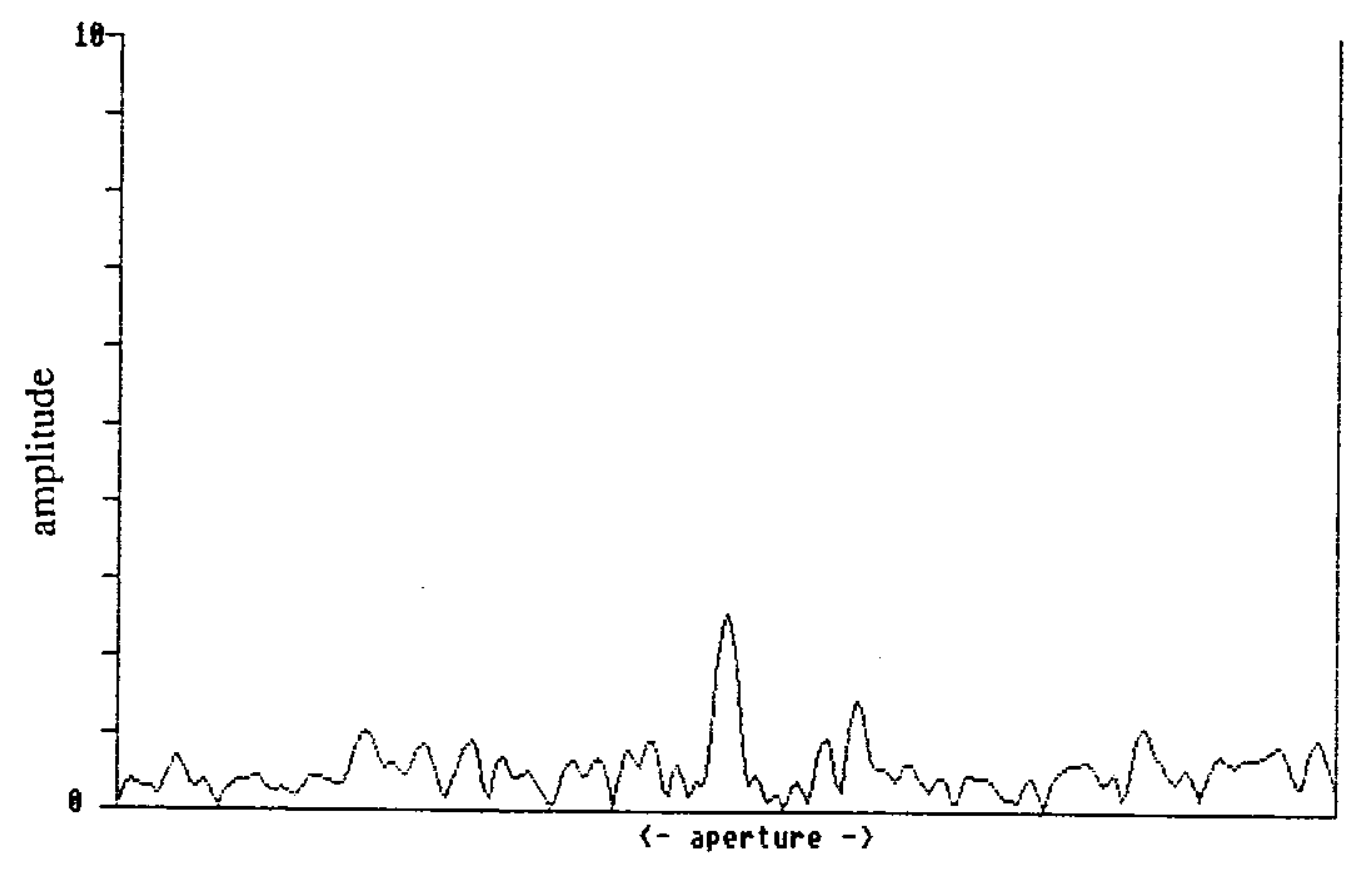

Fig, 4.7(c) Reconstructed Image with a Random Transducer Error Motion of $<0.8 \lambda$.

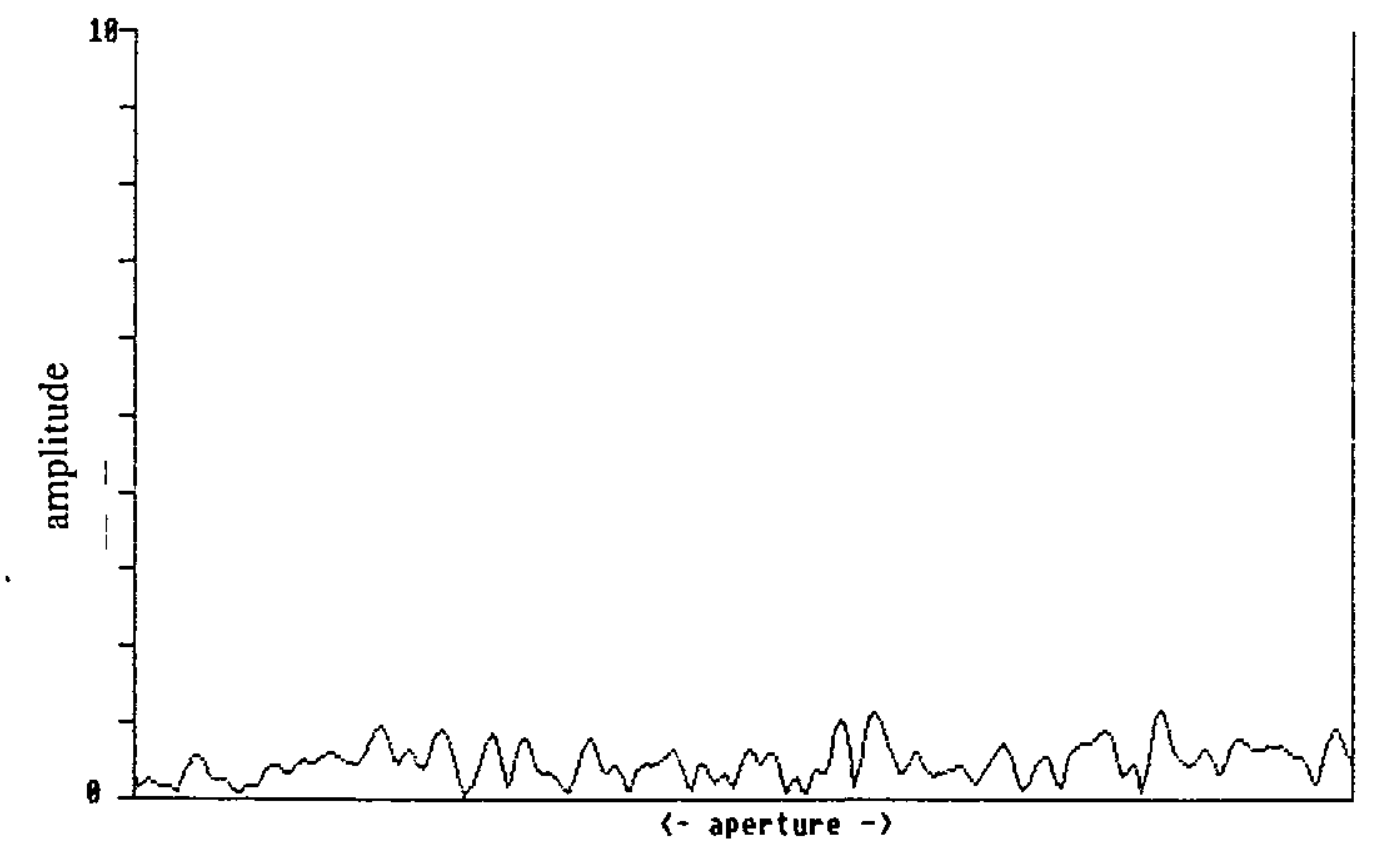

Fig, 4.7(d) Reconstructed Image with a Random Transducer Error Motion of $<1 \lambda$ 
the number of cycles of the error motion with respect to the aperture.

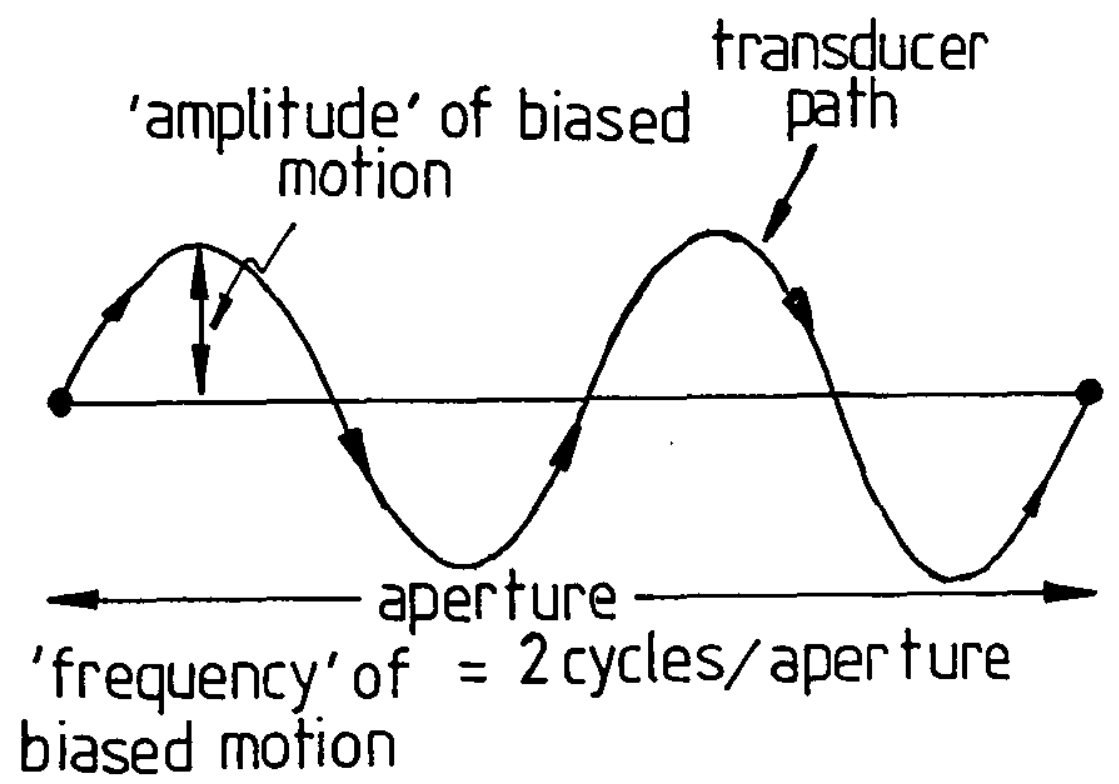

Fig, 4.8 Definition of Biased Error Motion Amplitude and Frequency

Fig. 4.9 shows the varlation of the received baseband quadrature signals. I and 0 , along the aperture de to two similar targets at a range of $10 \mathrm{~m}$, and with a separation of $0.5 \mathrm{~m}$. In this simulation both the transmitter and the receiver elements are moved along the aperture. The aperture length is $4.672 \mathrm{~m}$. Which is equivalent to $128 \lambda$. The operating frequency is $40 \mathrm{kHz}$ giving a wavelength, $\lambda$ of approx. $36 \mathrm{~mm}$. Fig. 4.10 illustrates the reconstructed targets.

When a biased error motion is applied to the transducer. the reconstructed Image is degraded. The effects of varying the blased error motion frequency with constant motion error amplitude is IIlustrated in Fig. 4.11 

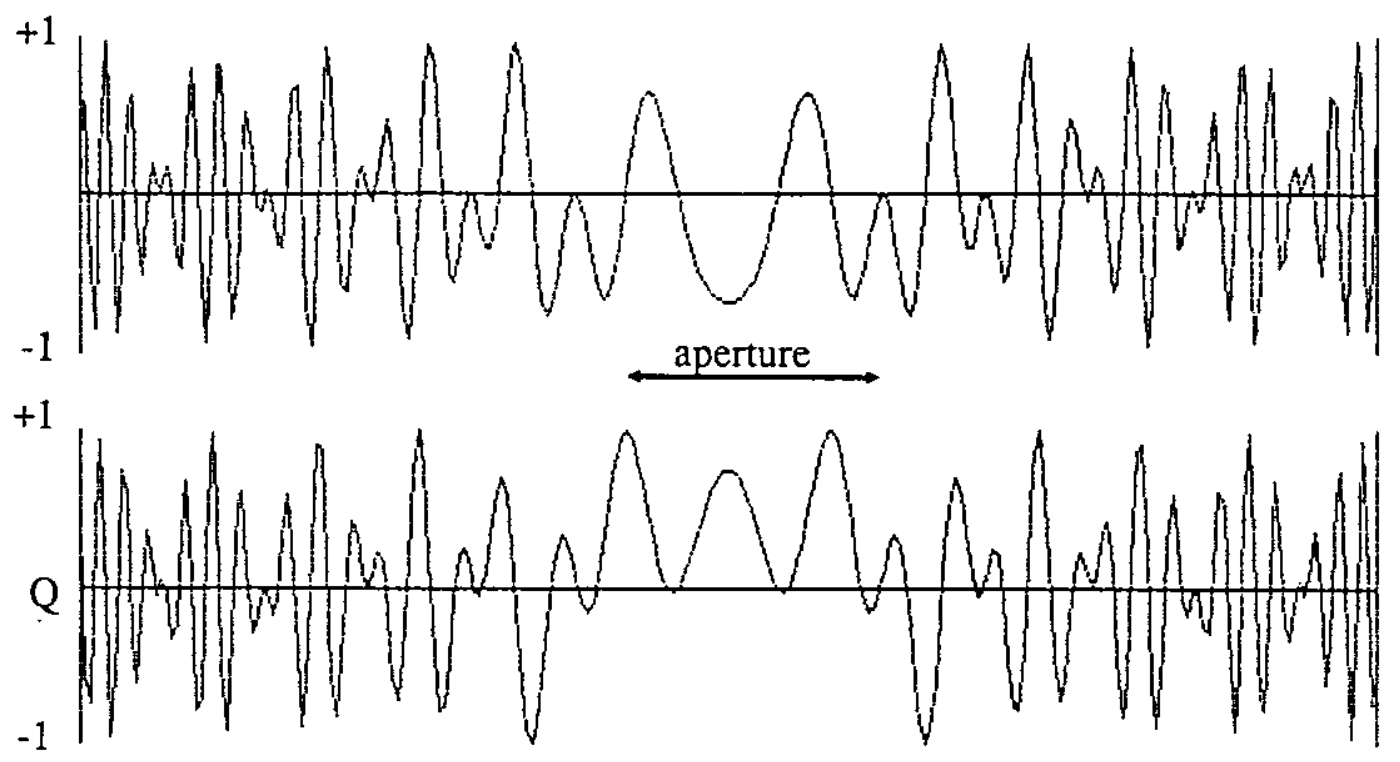

aperture

Fig 4.9 Variation of the Received Baseband Quadrature Signals across the Aperture due to a Paic of Targets at a Range of $10 \mathrm{~m}$.

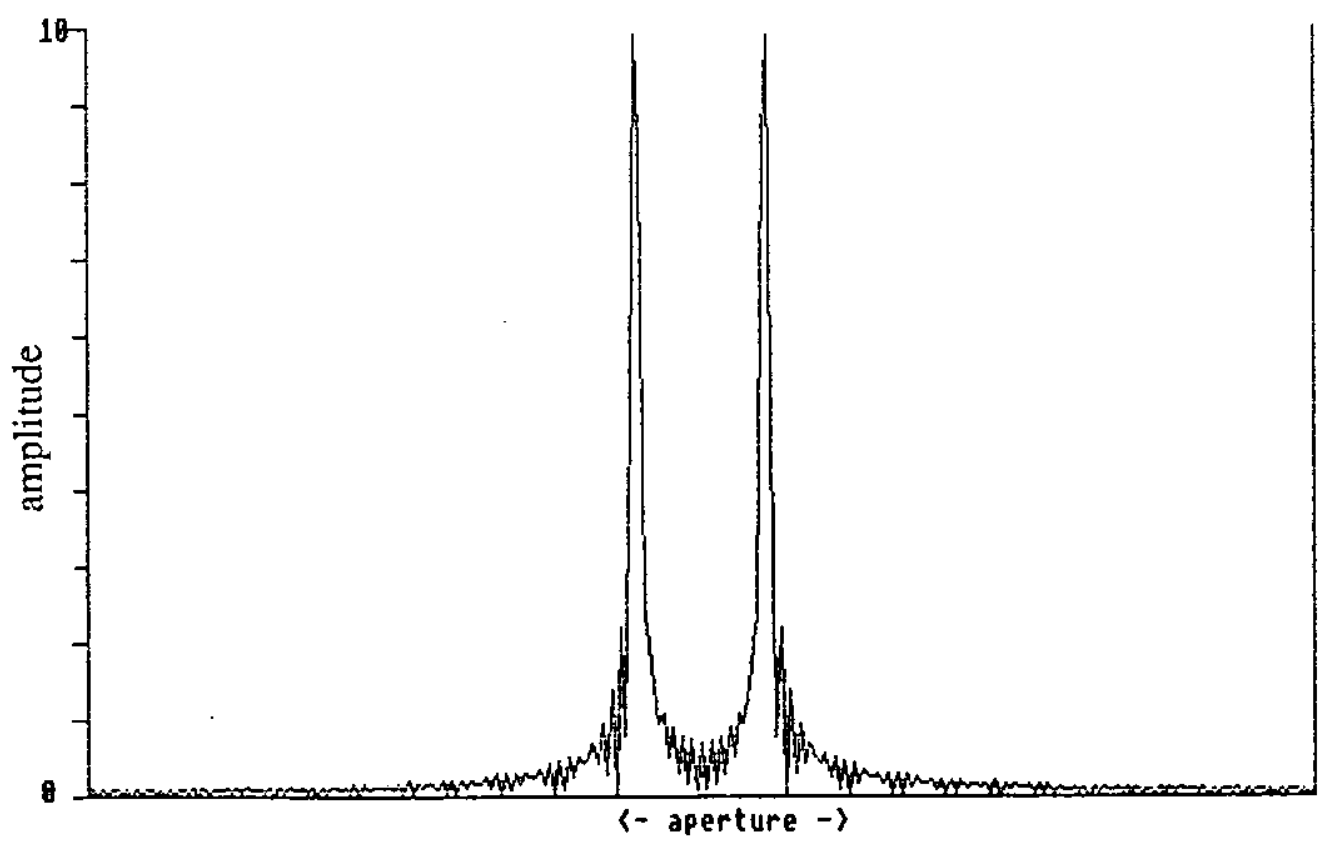

Fig, 4.10 Reconstructed Image of the pair of Targets. 
to Fig. 4.15. With an error motion amplitude of $0.01 \lambda$. and a frequency of biased error motion of 0.5 cycles/ aperture, Fig. 4.11(a) shows that there is a slight migration in the target position and only a slight decrease in the amplitude of the target. When the blased error motion frequency is increased to 1 cycle/aperture, as lliustrated in Fig. 4.11(b). spurious targets are generated. Increasing the frequency of the biased error motion to 5 cycles/aperture in Fig. 4.11(c). 10 cycles/ operture in Fig. $4.11(d)$ and 20 cycles/aperture in Fig. $4.11(e)$ results in target migration and the generation of spurious targets. Fig. $4.11(f)$ shows image reconstructed for an error motion frequency of 50 cycles/aperture. The targets have been reconstructed. although with a lower amplitude and increased side lobes.

Fig. 4.12 illustrates the effects of increasing the error motion amplitude to $0.05 \lambda$ and varying the frequency of the error motion. When the frequency of the error motion is less than 0.5 cycles/aperture the targets are reconstructed but spurious targets are generated and target migration occurs as the frequency approaches 0.5 cycles/ aperture. Increasing the frequency results in the torgets not being reconstructed but Fig. $4.12(\mathrm{~g})$ shows the targets are reconstructed, although in the presence of spurious targets, when the frequency is increased to 50 cycles/ aperture.

Increasing the error motion amplitude to $0.1 \lambda .0 .5 \lambda$ and $1 \lambda$ results in the targets only being recognisable when the frequency is much less than $0.5 \lambda$ cycles/aperture as illustrated in Fig. 4.13. Fig. 4.14 and Fig. 4.15 respectively.

We conclude that the reconstructed images from a 


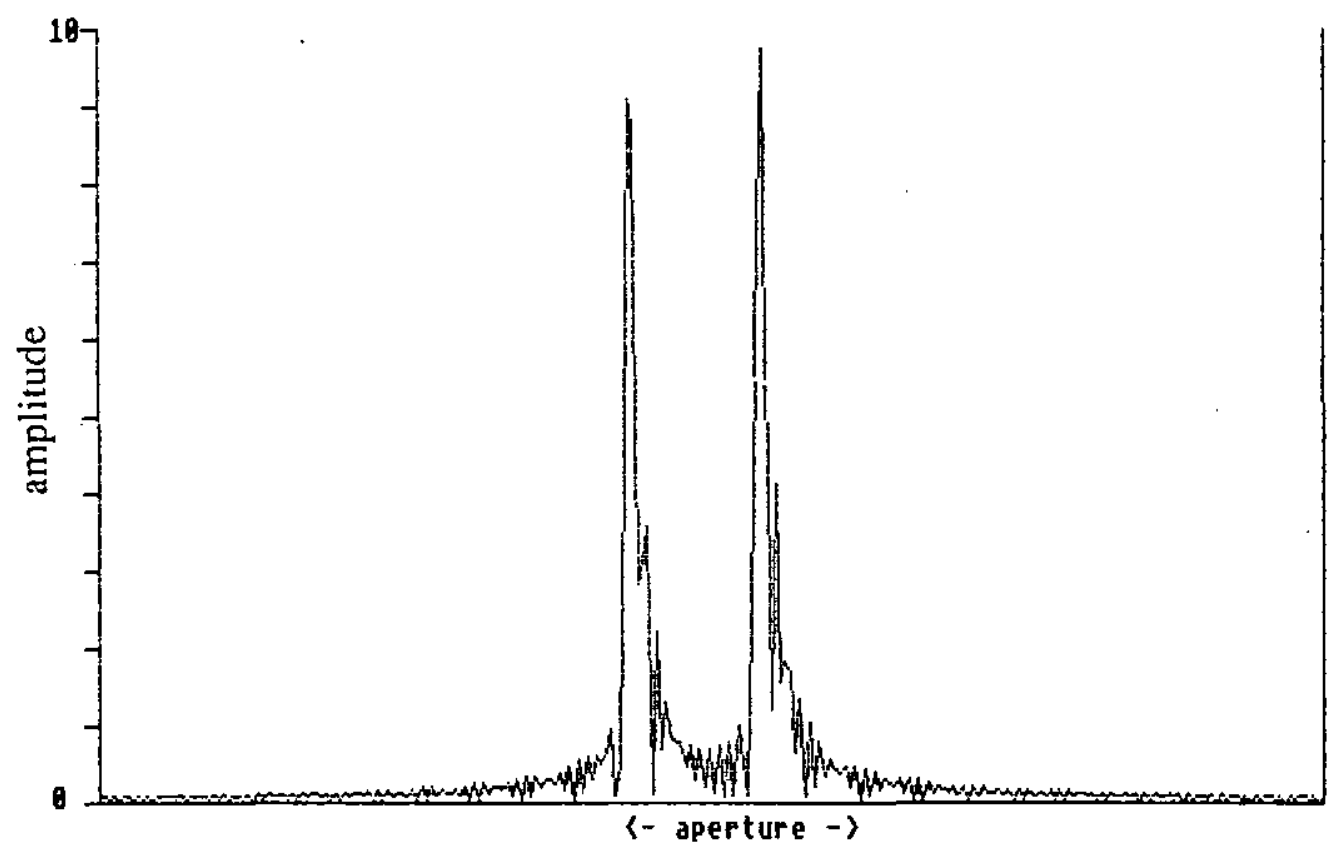

Eig. 4.11(a) Reconstructed Targets with a Biased Transducer Error Motion of Amplitude $0.01 \lambda$ and a Erequency of 0.5 cycles/aperture.

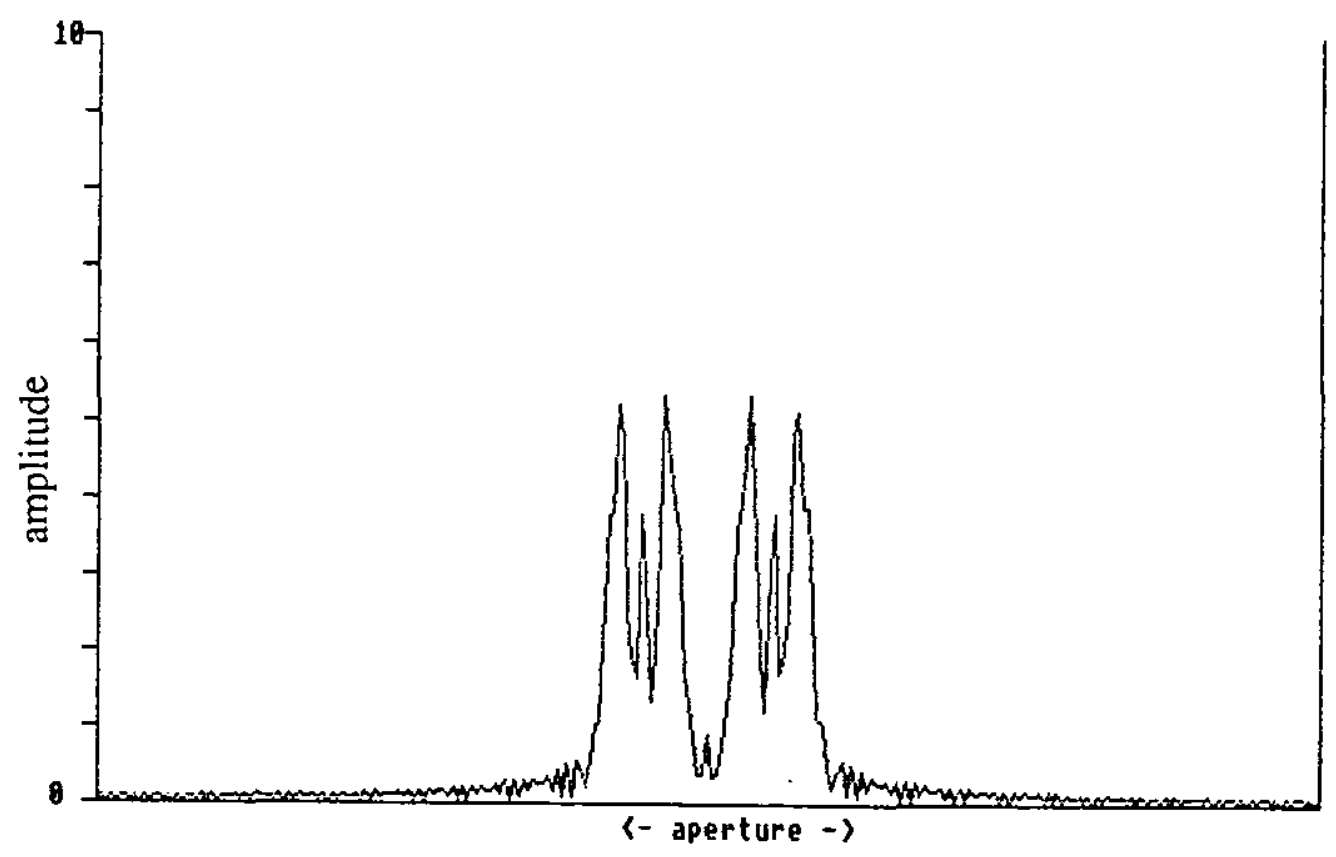

Eig. 4.11(b) Reconstructed Targets with a Biased Transducer Error Motion of Amplitude $0.01 \lambda$ and a Frequency of 1 cycles/aperture. 


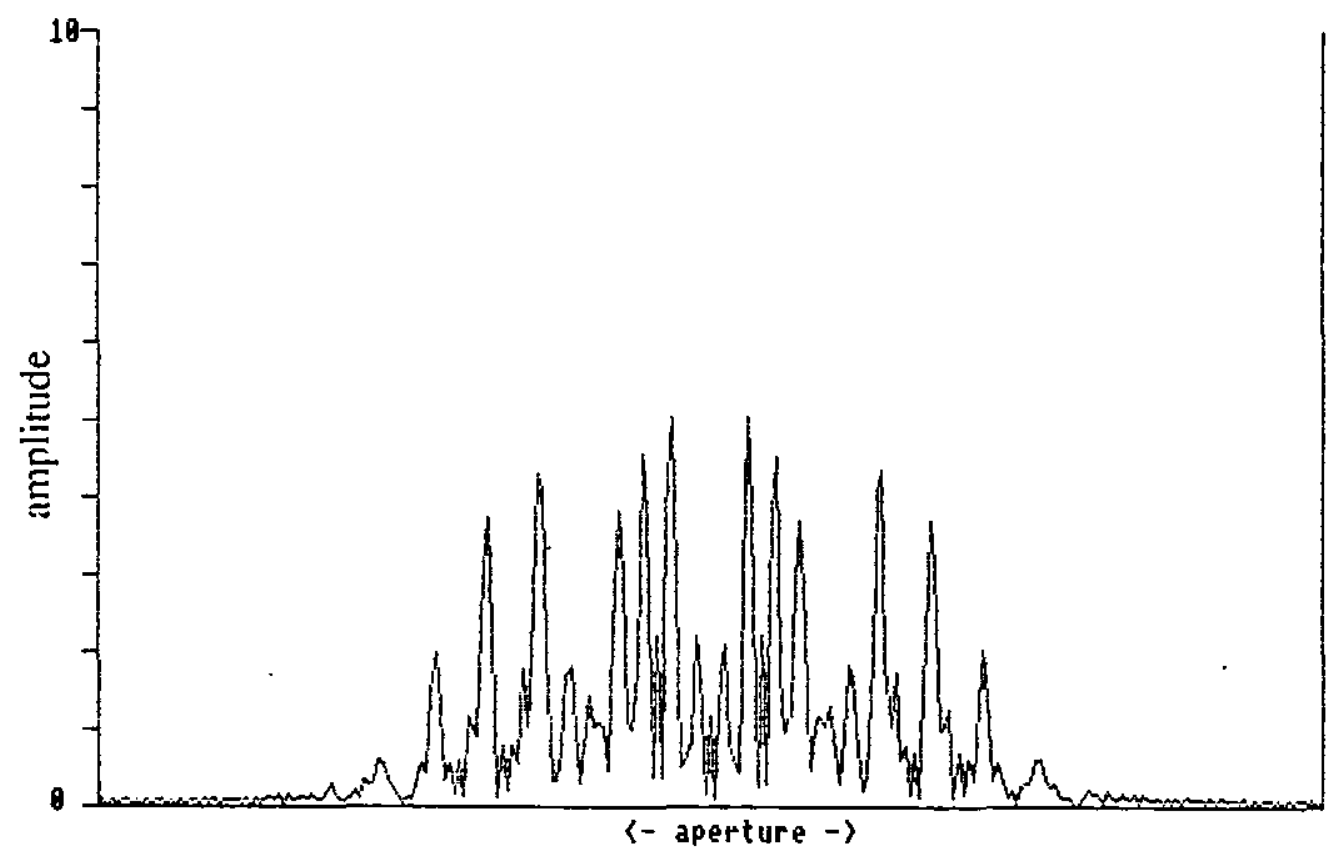

Fig. 4.11(c) Reconstructed Targets with a Biased Transducer Error Motion of Amplitude $0.01 \lambda$ and a Frequency of 5 cycles/aperture.

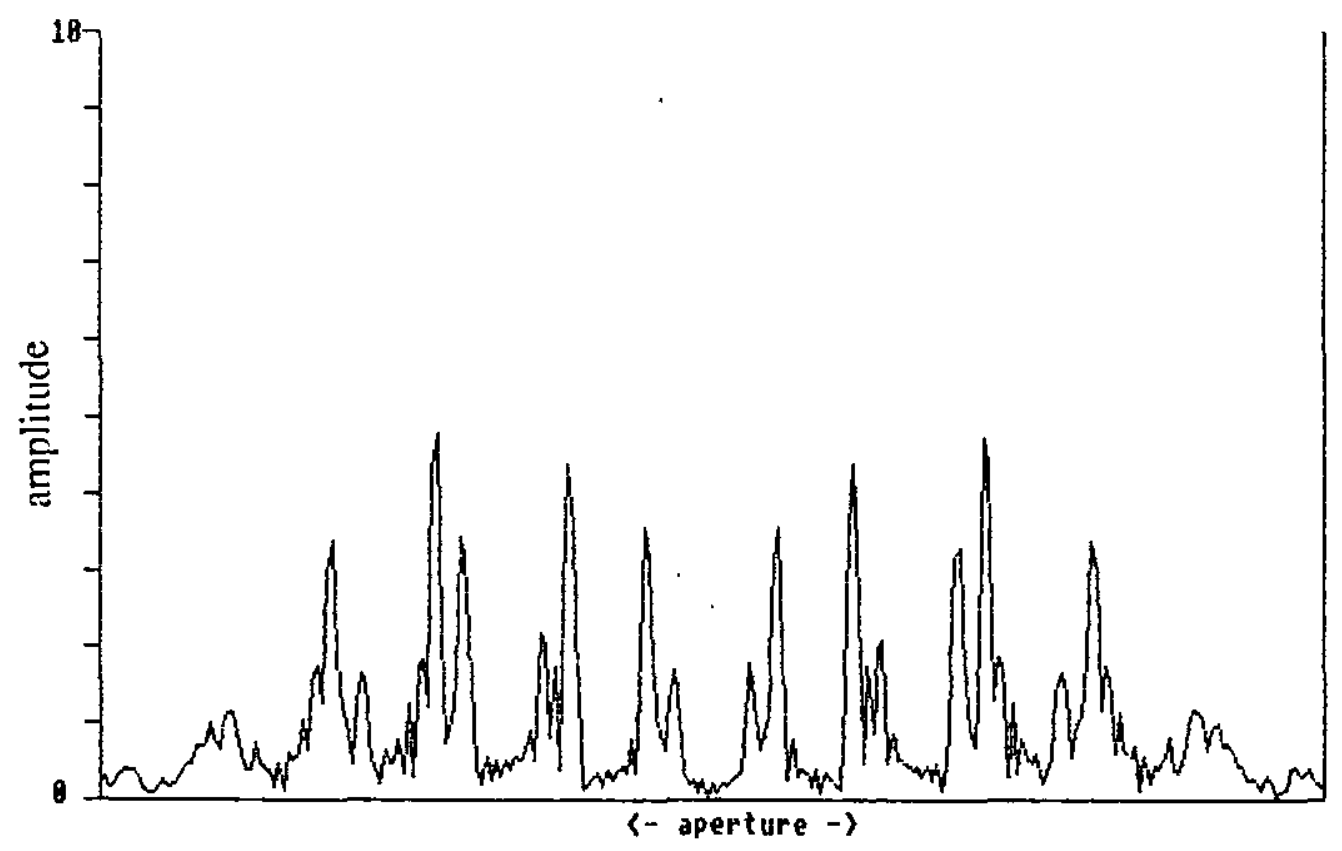

Fig. 4.11(d) Reconstructed Targets with a Biased Transducer Error Motion of Amplitude $0.01 \lambda$ and a Frequency of 10 cycles/aperture. 


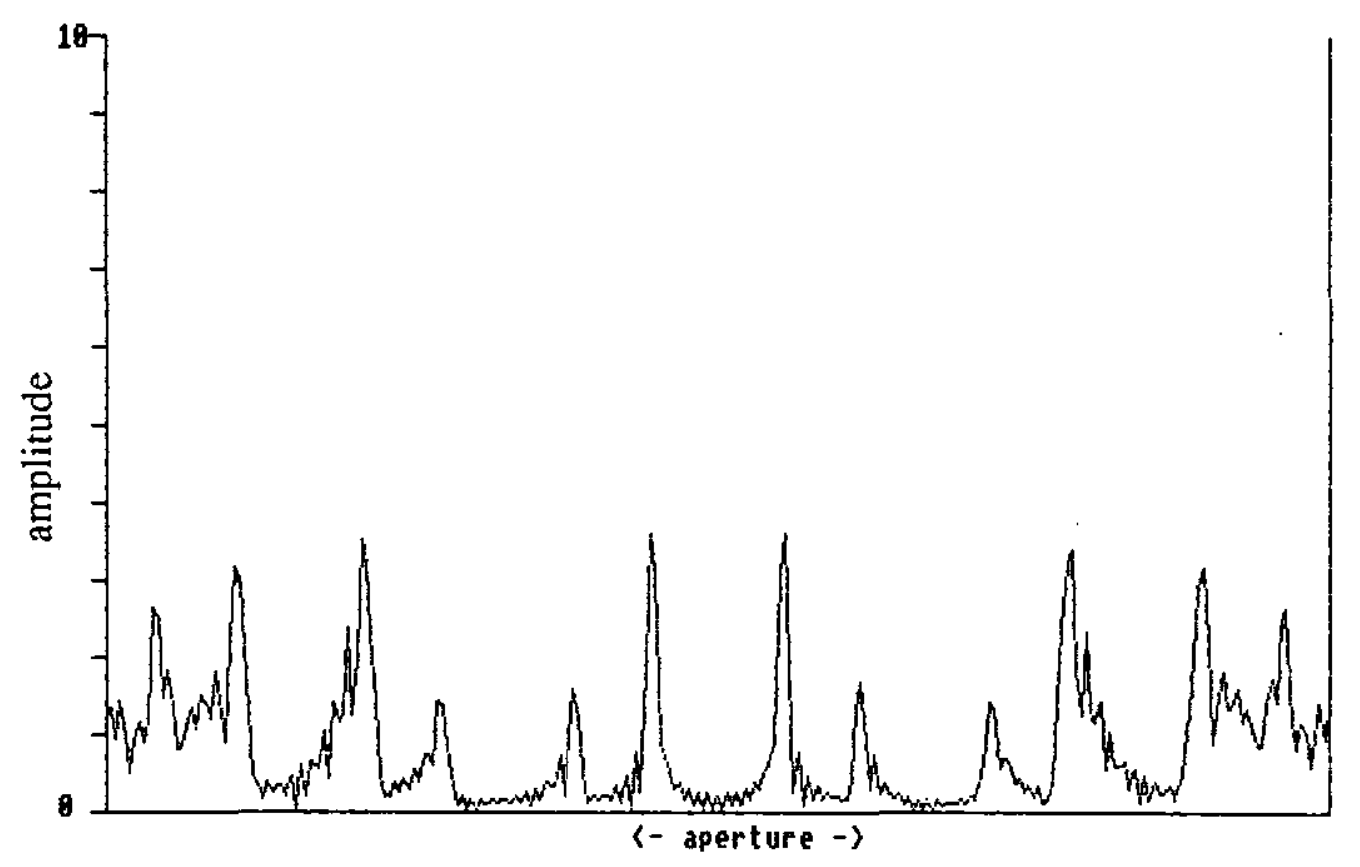

Fig. 4.11(e) Reconstructed Targets with a Biased Transducer Error Motion of Amplitude $0.01 \lambda$ and a Frequency of 20 cycles/aperture.

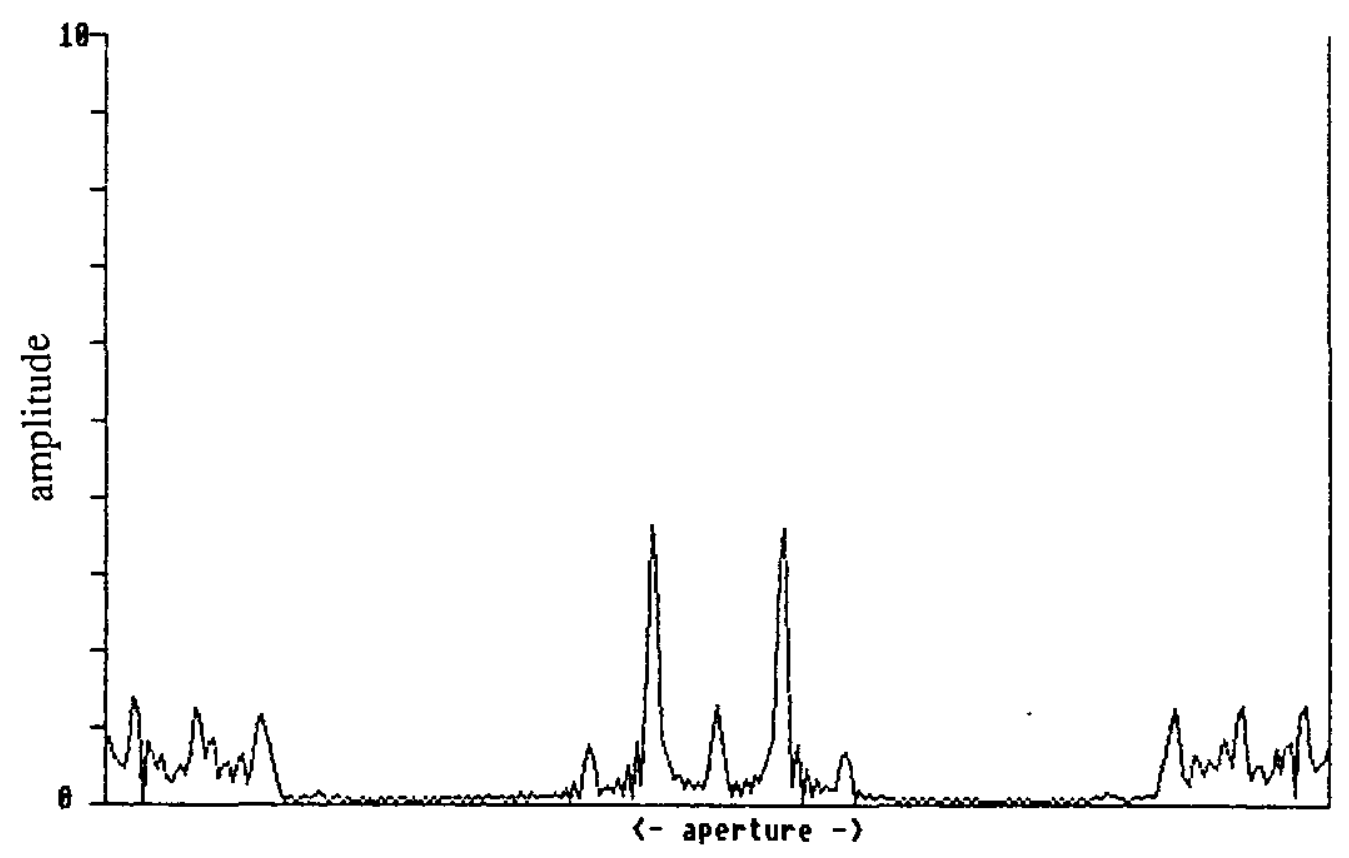

Fig, 4.11(f) Reconstructed Targets with a Biased Transducer Error Motion of Amplitude $0.01 \lambda$ and a Frequency of 50 cycles/aperture. 


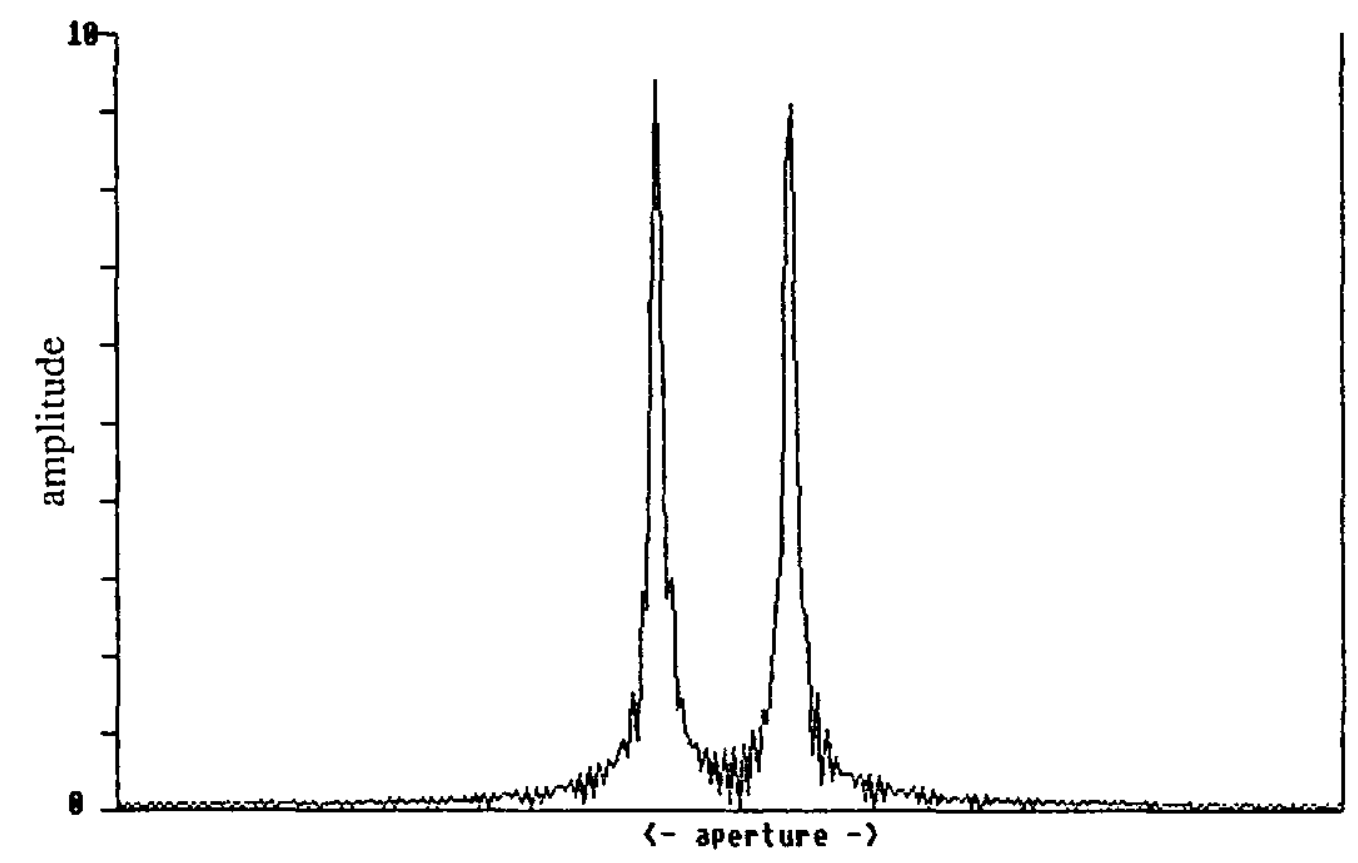

Fig, 4.12(a) Reconstructed Targets with a Biased Transducer Error Motion of Amplitude $0.05 \lambda$ and a Frequency of 0.1 cycles/aperture.

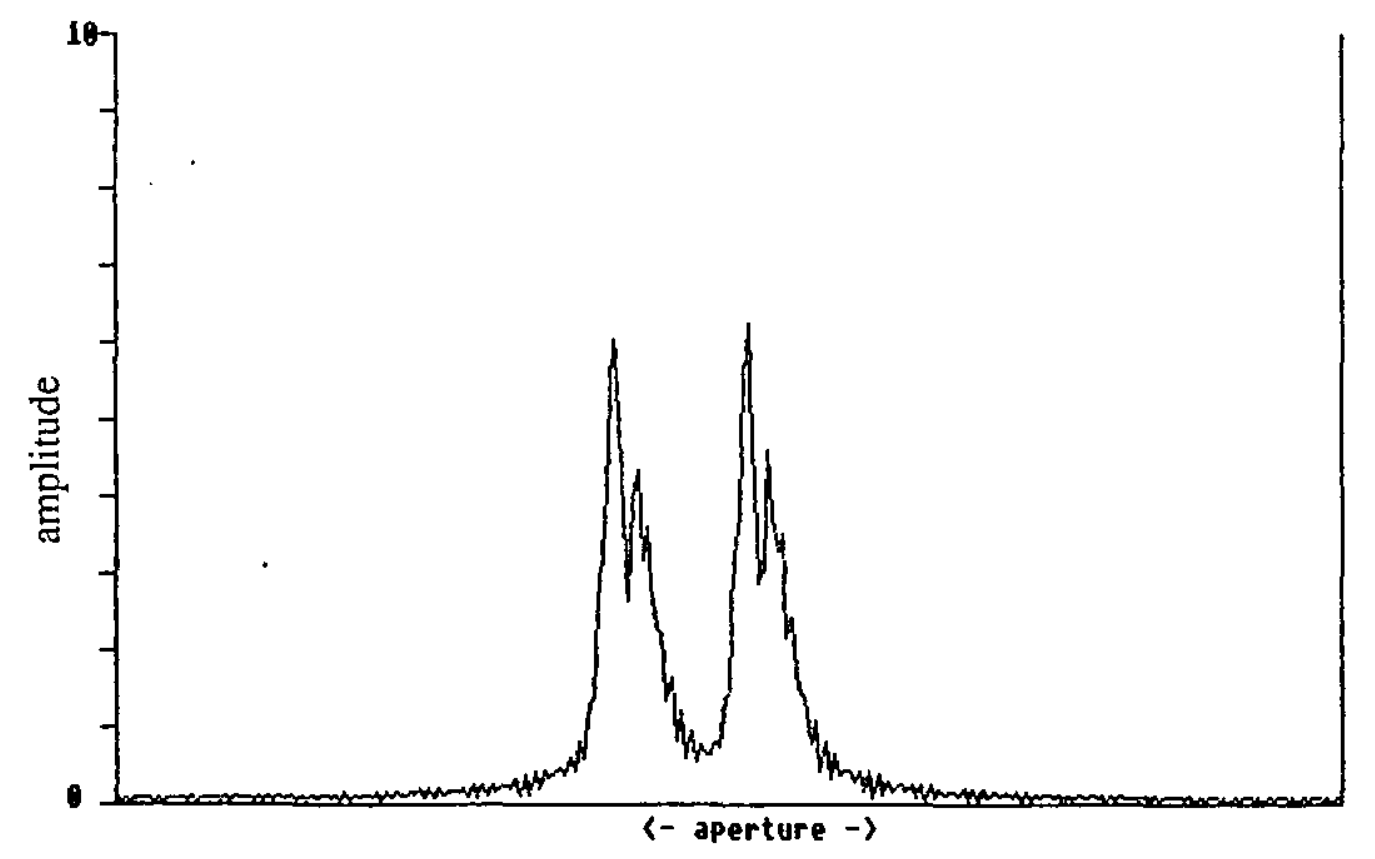

Fig, 4.12(b)_Reconstructed Targets with a Biased Transducer Error Motion of Amplitude $0.05 \lambda$ and a Frequency of 0.3 cycles/aperture. 


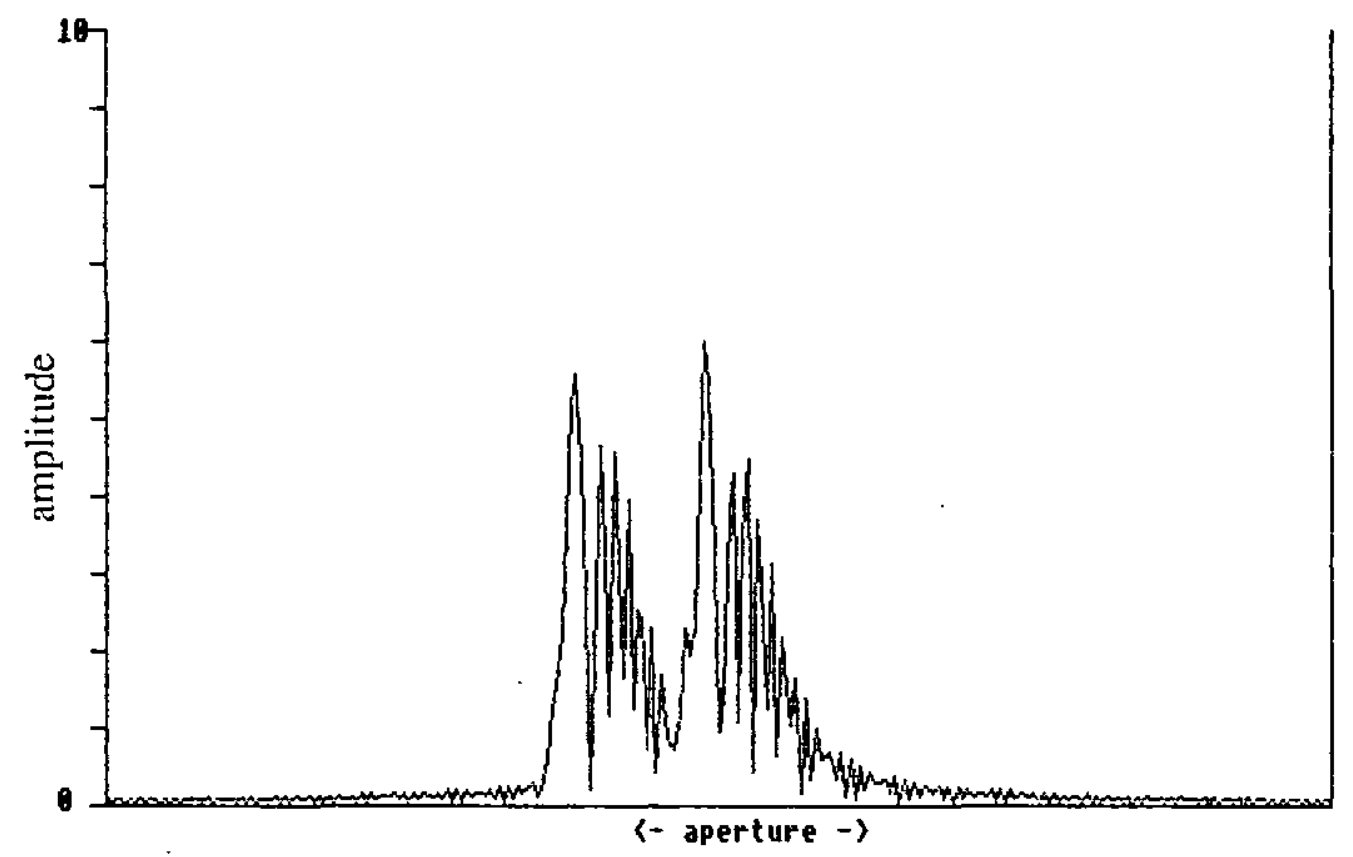

Fig, 4.12(c) Reconstructed Targets with a Biased Transducer Error Motion of Amplitude $0.05 \lambda$ and a Frequency of 0.5 cycles/aperture.

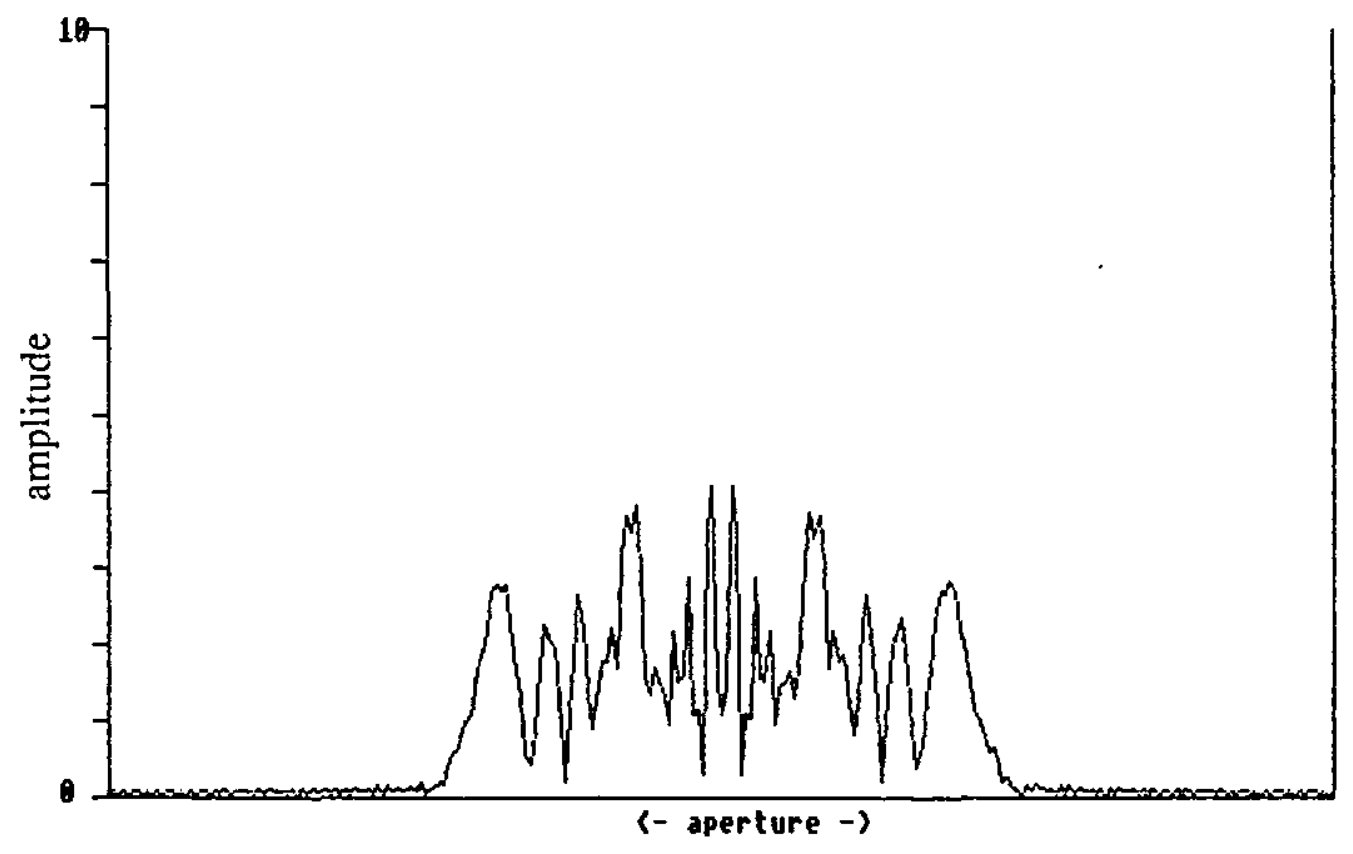

Fig. 4.12(d) Reconstructed Targets with a Biased Transducer Error Motion of Amplitude $0.05 \lambda$ and a Frequency of 1 cycles/aperture. 


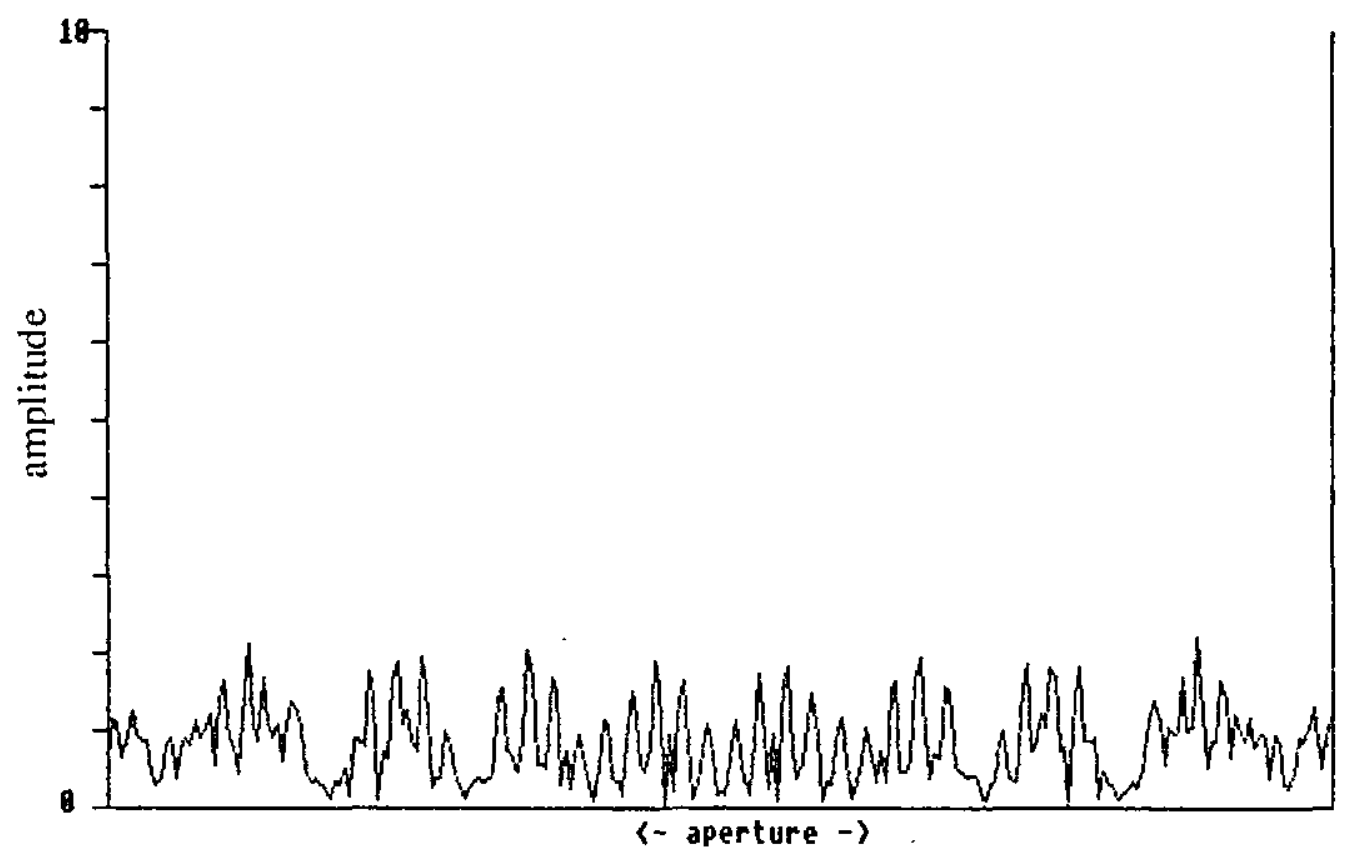

Fig. 4.12(e) Reconstructed Targets with a Biased Transducer Error Motion of Amplitude $0.05 \lambda$ and a Erequency of 5 cycles/aperture.

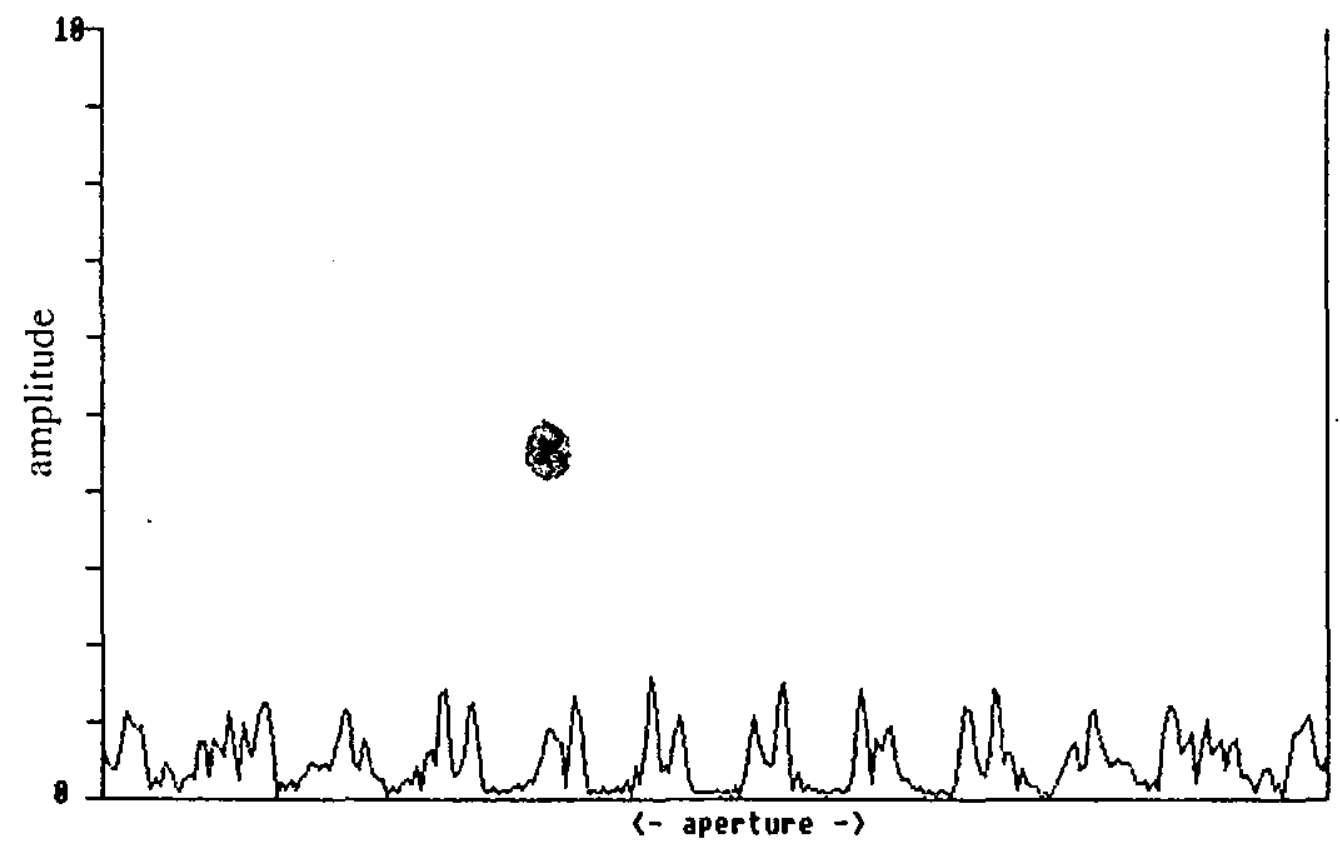

Fig. 4.12(f)_Reconstructed Targets with a Biased Transducer Error Motion of Amplitude $0.05 \lambda$ and a Erequency of 10 cycles/aperture. 


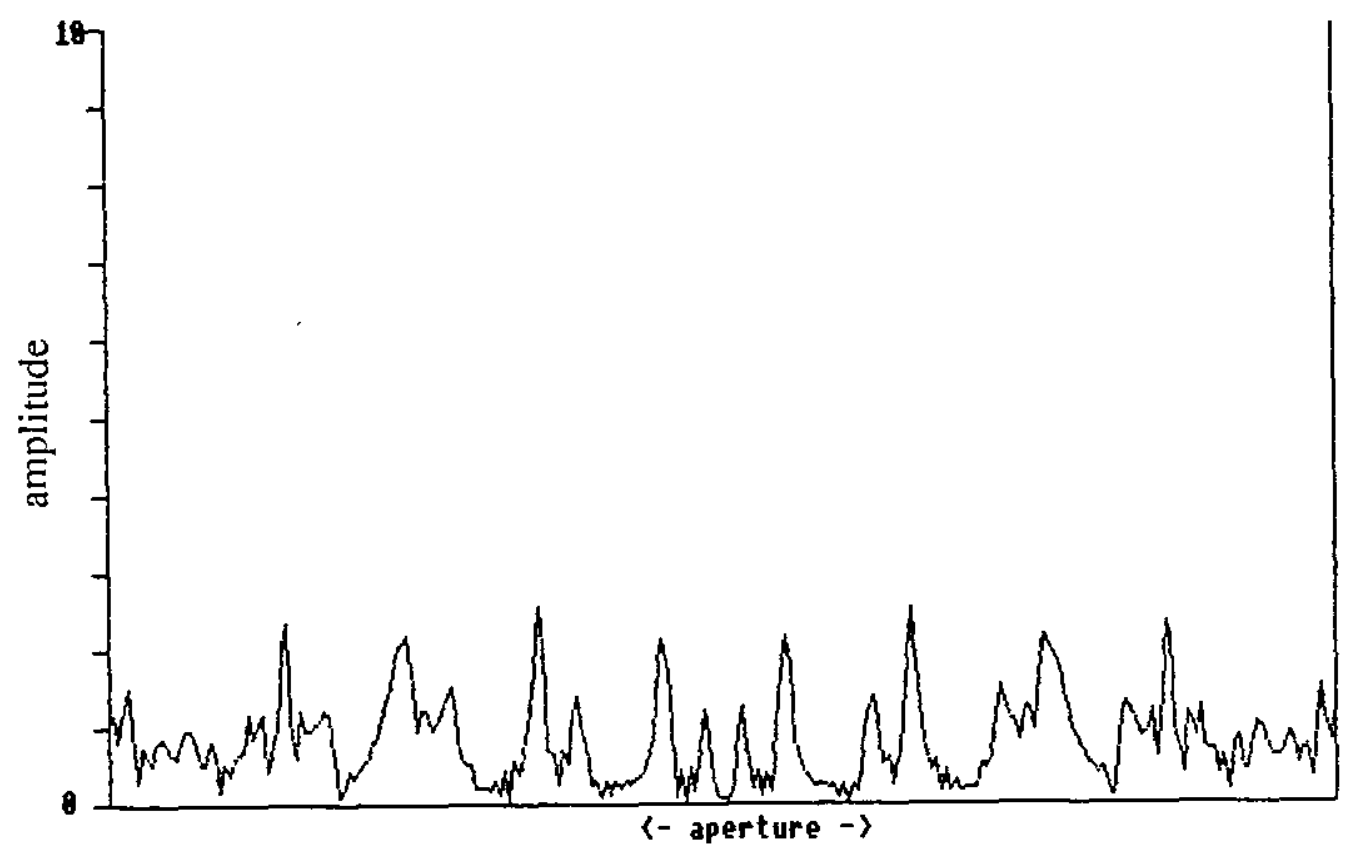

Fig. 4.12(g) Reconstructed Targets with a Biased Transducer Error Motion of Amplitude $0.05 \lambda$ and a Frequency of 50 cycles/aperture. 


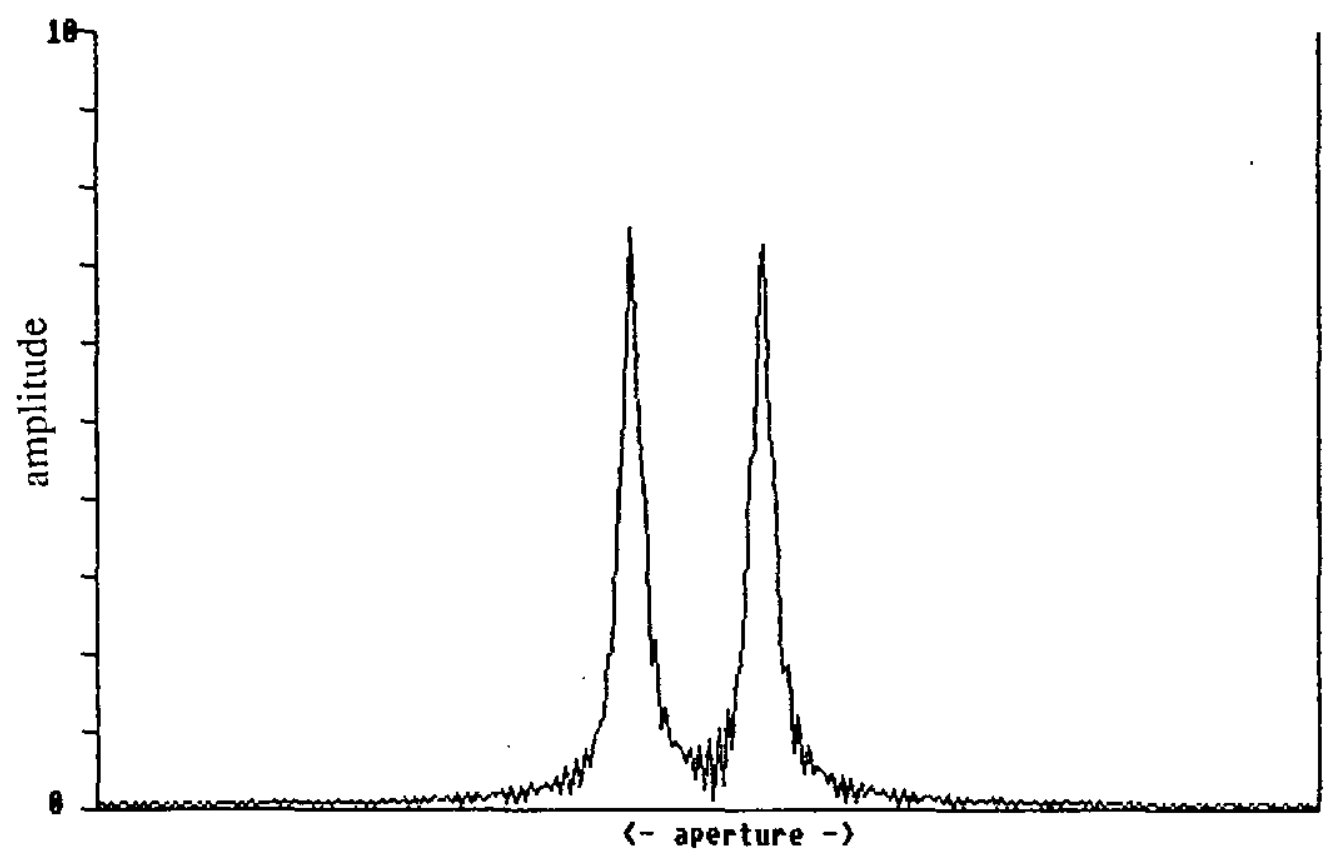

Fig, 4.13(a) Reconstructed Targets with a Biased Transducer Error Motion of Amplitude $0.1 \lambda$ and a Frequency of 0.1 cycles/aperture.

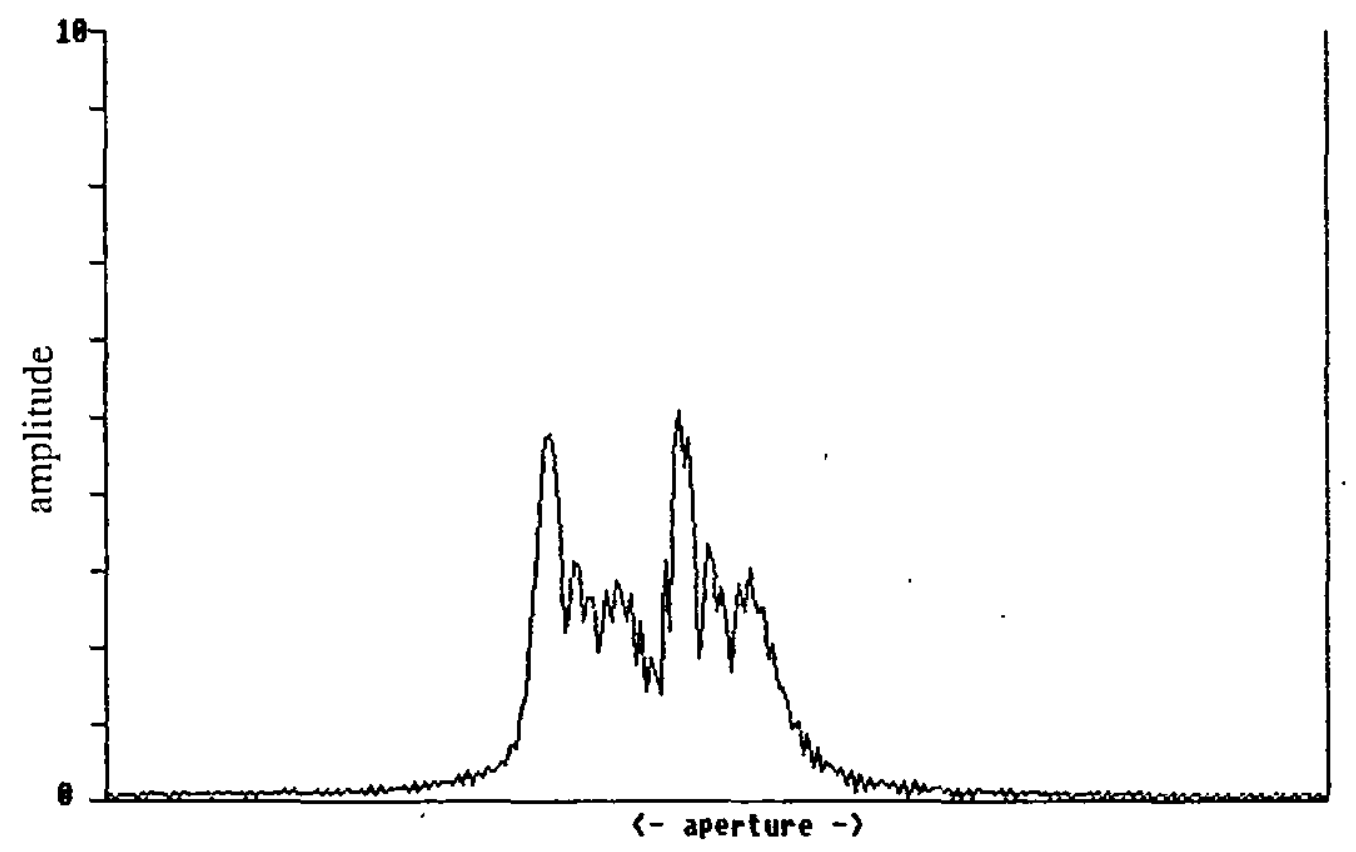

Fig. 4.13(b) Reconstructed Targets with a Biased Transducer Error Motion of Amplitude $0.1 \lambda$ and a Frequency of 0.3 cycles/aperture. 


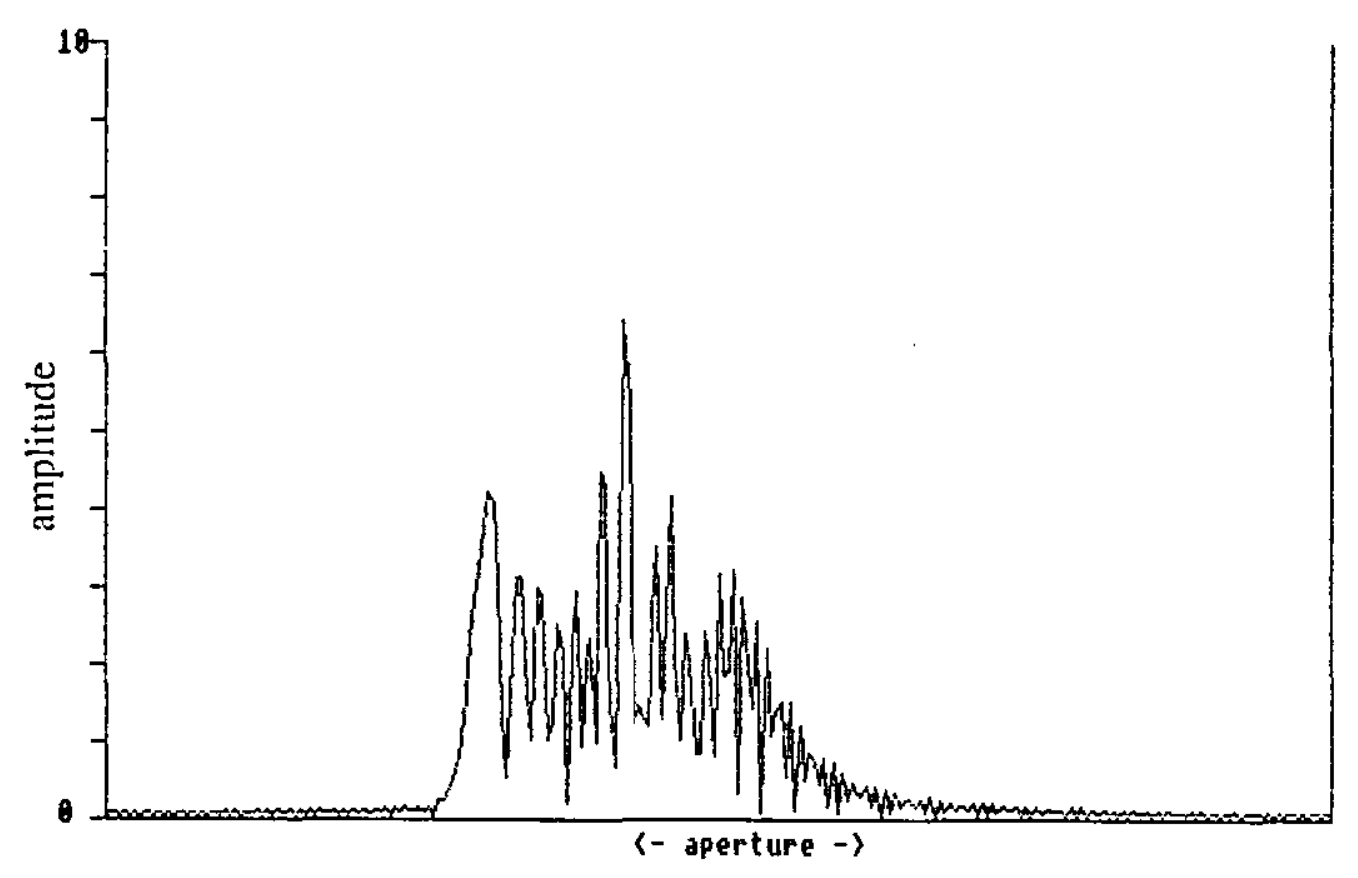

Fig, 4.13(c) Reconstructed Targets with a Biased Transducer Error Motion of Amplitude $0.1 \lambda$ and a Frequency of 0.5 cycles/aperture.

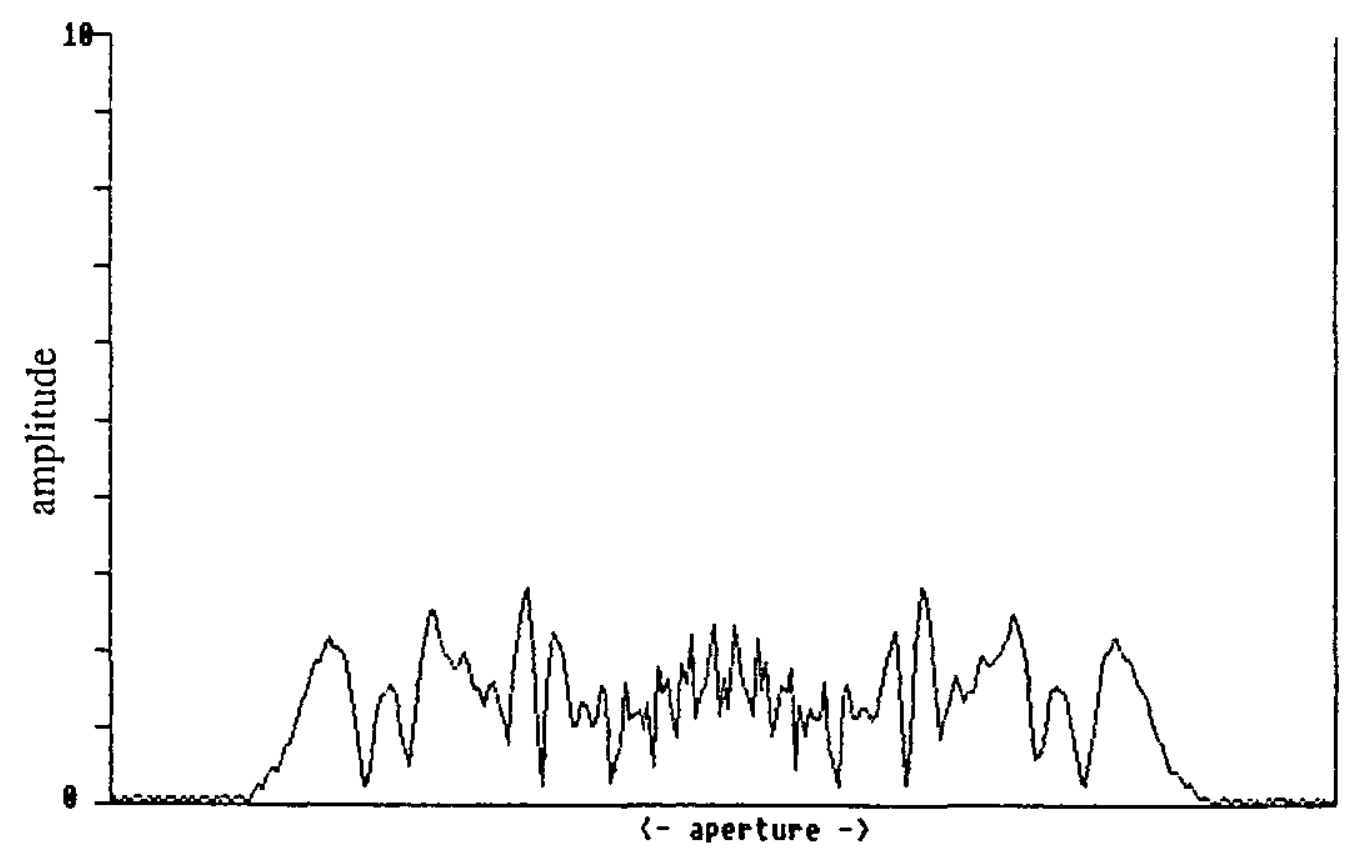

Fig. 4.13(d) Reconstructed Targets with a Biased Transducer Error Motion of Amplitude $0.1 \lambda$ and a Frequency of 1 cycles/aperture. 


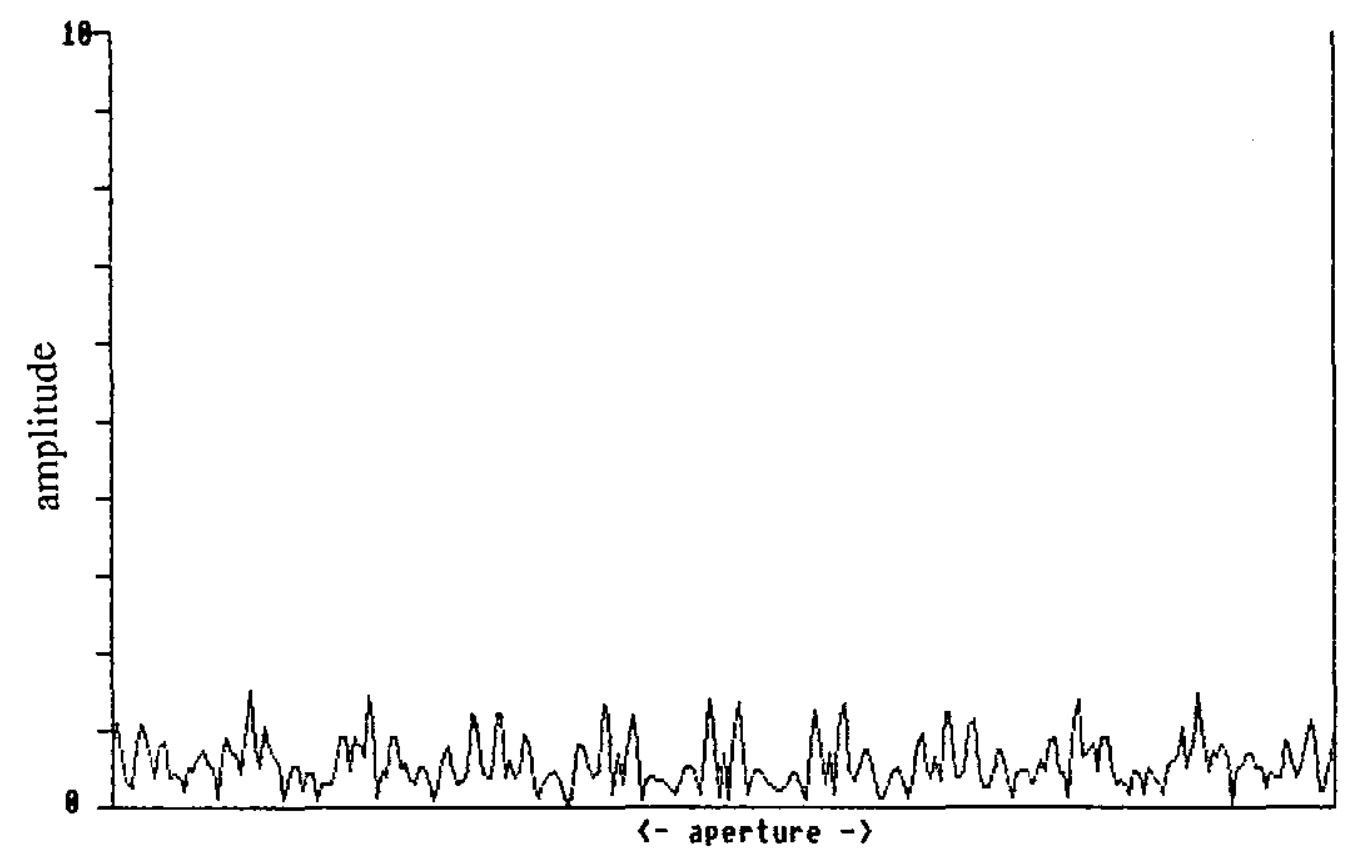

Fig. 4.13(e) Reconstructed Targets with a Biased Transducer Error Motion of Amplitude $0.1 \lambda$ and a Frequency of 5 cycles/aperture.

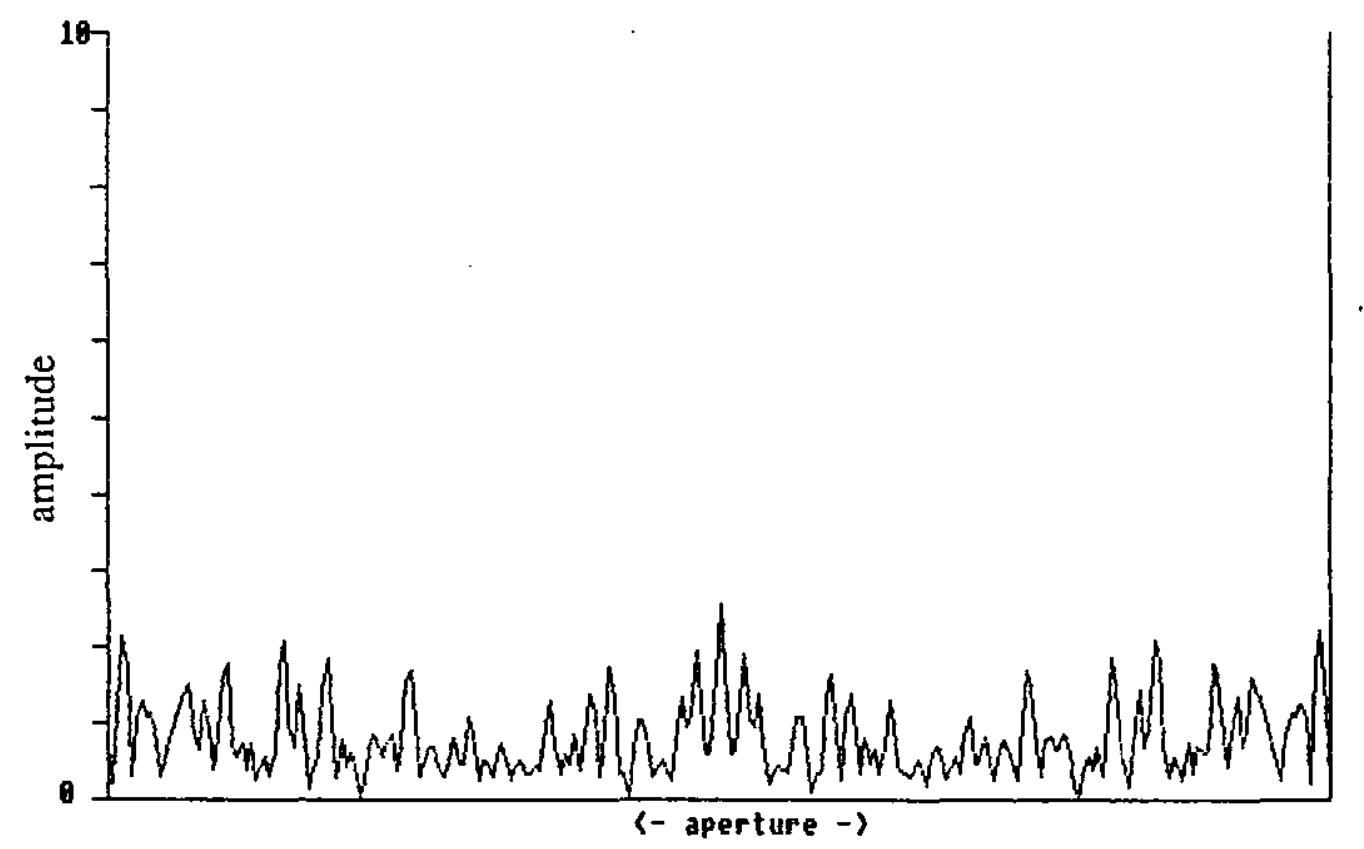

Fig, 4.13(f) Reconstructed Targets with a Biased Transducer Error Motion of Amplitude $0.1 \lambda$ and a Frequency of 10 cycles/aperture. 


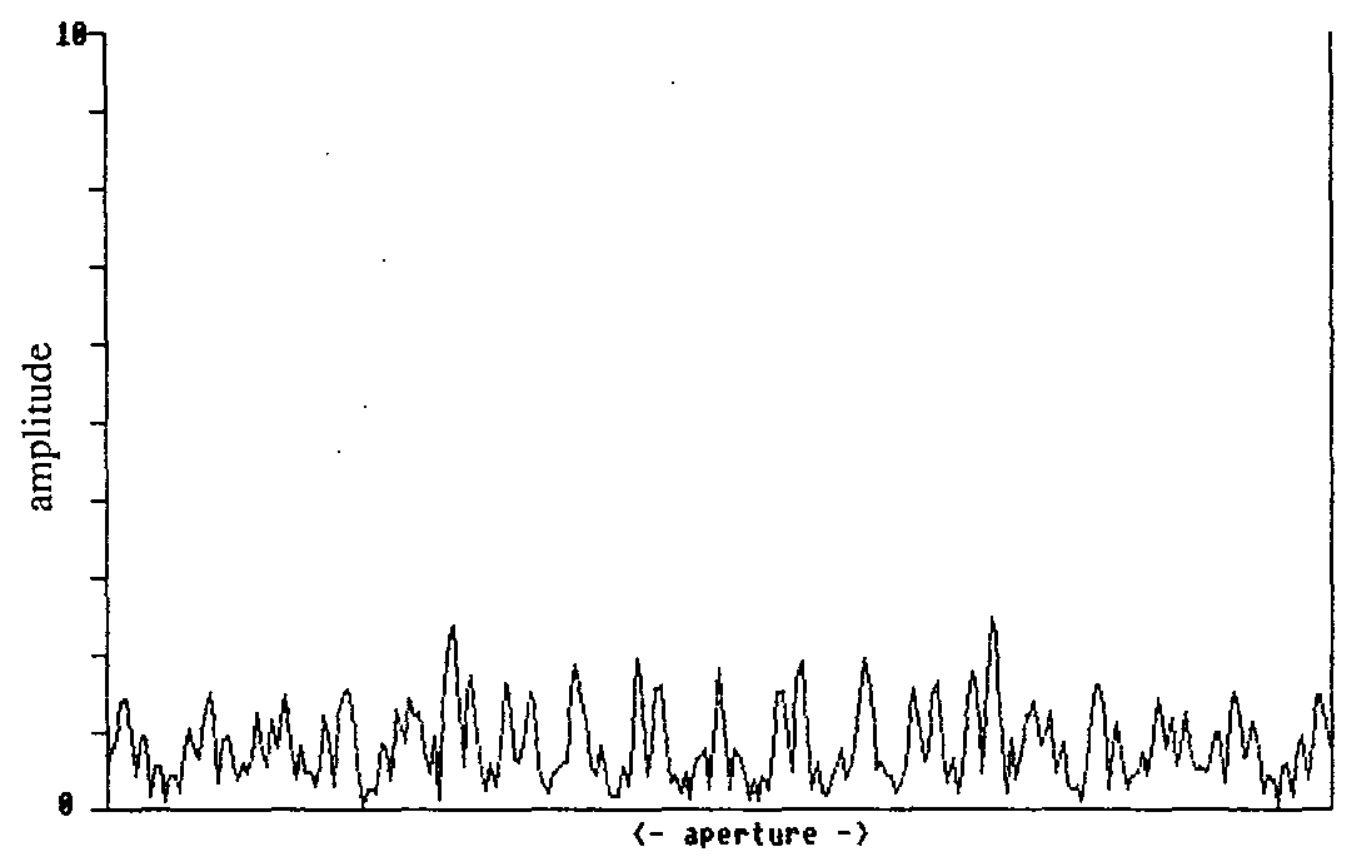

Fig. 4.13(g) Reconstructed Targets with a Biased Transducer Error-Motion of Amplitude $0.1 \lambda$ and a Frequency of 50 cycles/aperture.

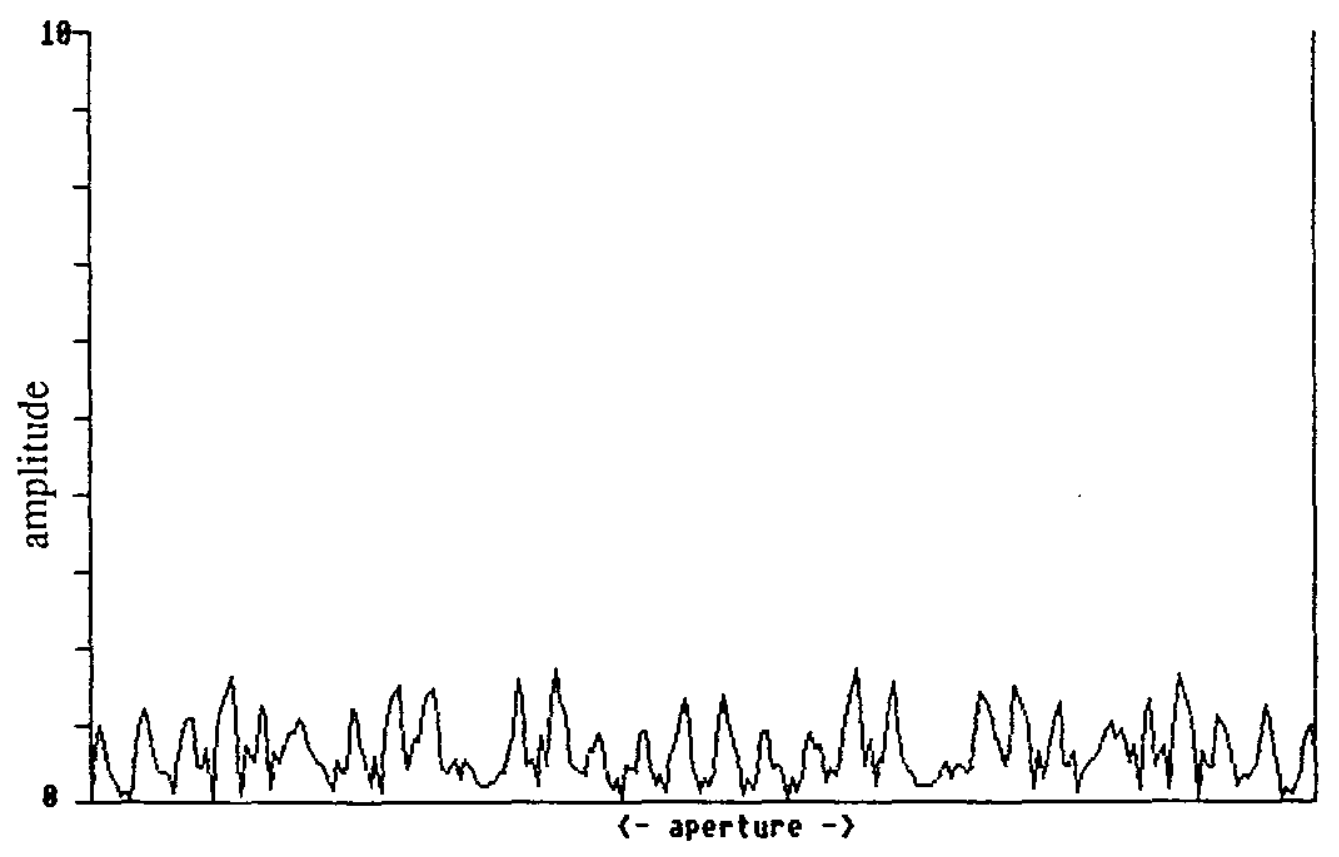

Eig. 4.13(h) Reconstructed Targets with a Biased Transducer Error Motion of Amplitude $0.1 \lambda$ and a Frequency of 100 cycles/aperture. 


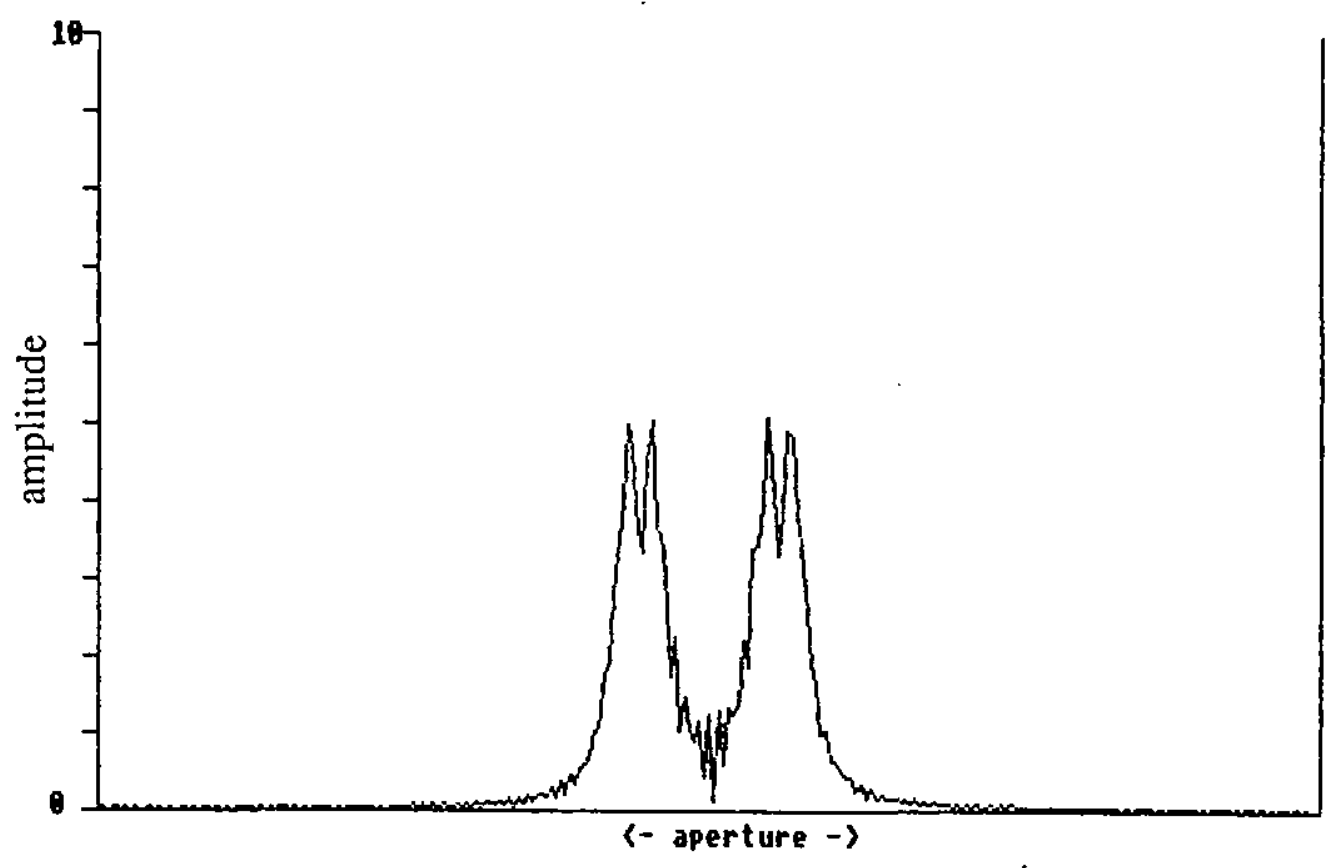

Fig, 4.14(a) Reconstructed Targets with a Biased Transducer Error Motion of Amplitude $0.5 \lambda$ and a Frequency of 0.01 cycles/aperture.

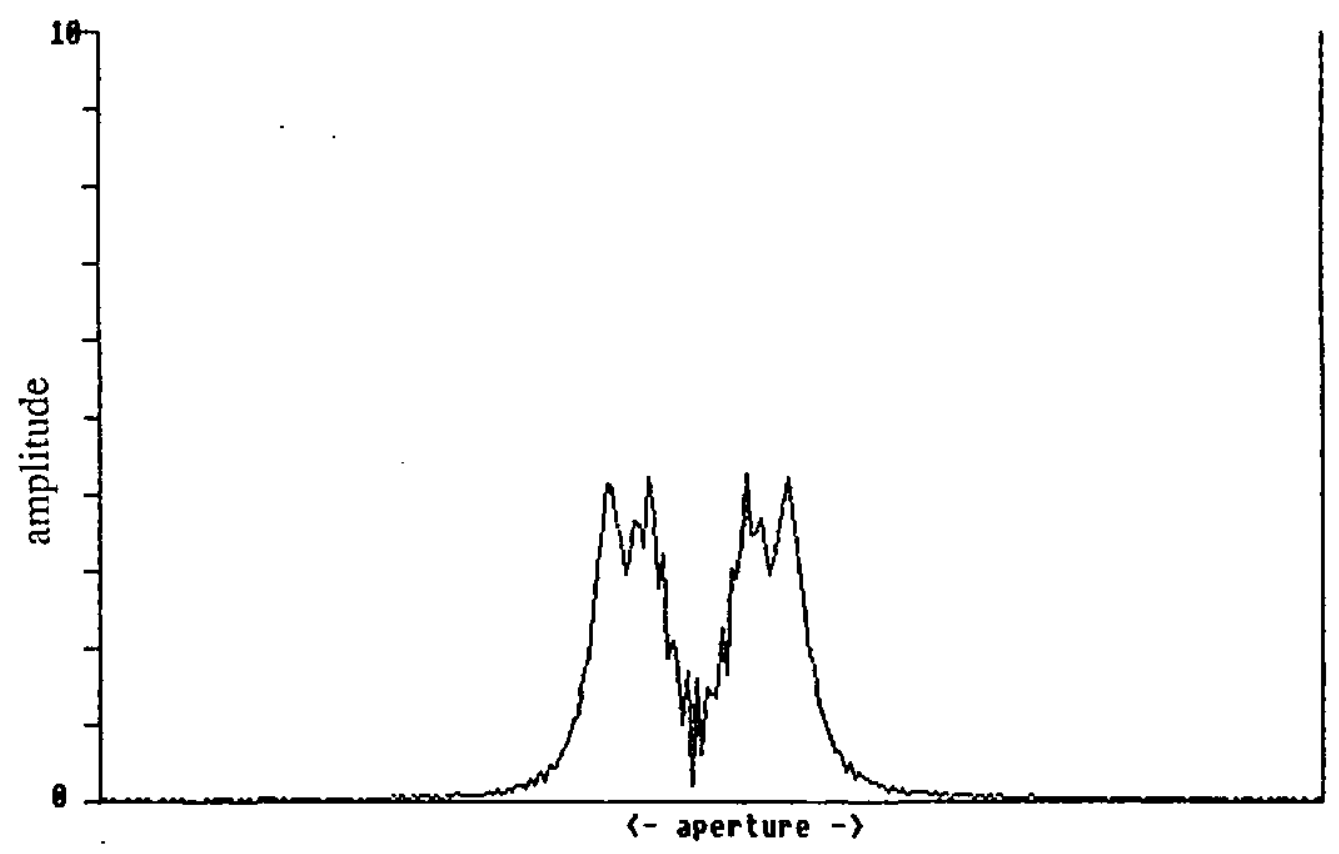

Fig. 4.14(b)_Reconstructed Targets with a Biased Transducer Error Motion of Amplitude $0.5 \lambda$ and a Frequency of 0.05 cycles/aperture. 


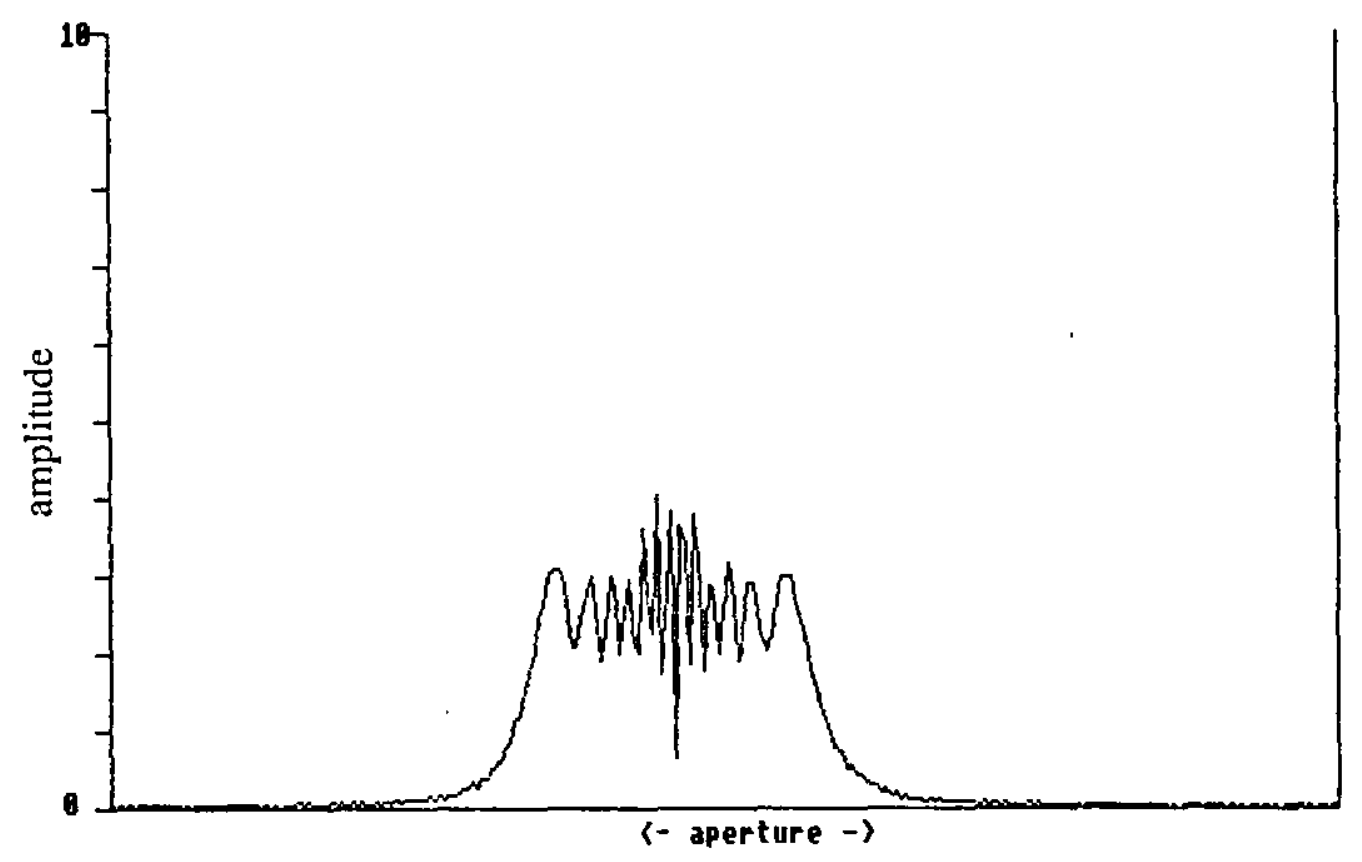

Fig. 4.14(c) Reconstructed Targets with a Biased Transducer Error Motion of Amplitude $0.5 \lambda$ and a Frequency of 0.1 cycles/aperture.

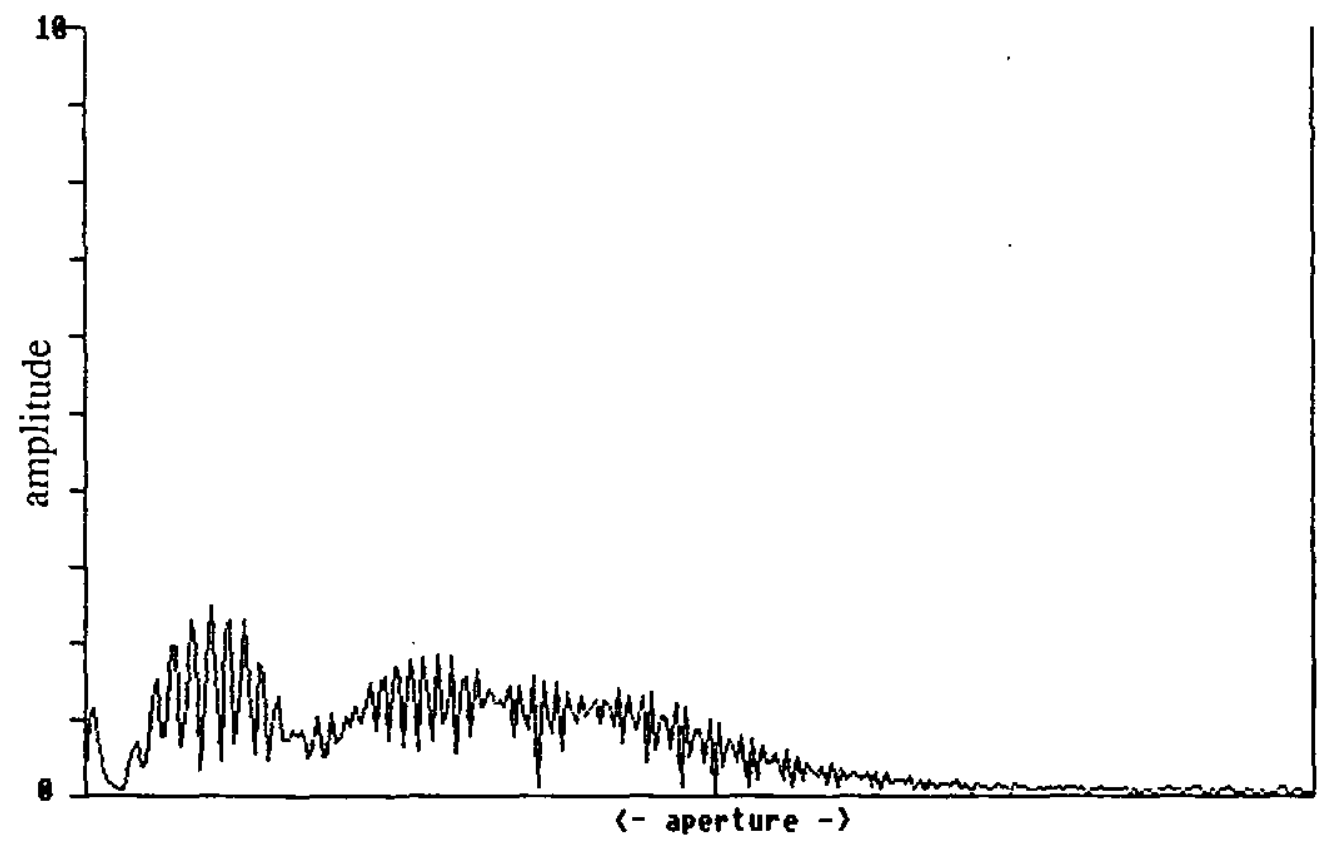

Fig. 4.14(d) Reconstructed Targets with a Biased Transducer Error Motion of Amplitude $0.5 \lambda$ and a Frequency of $0.5 \mathrm{cycles} / \mathrm{aperture.}$ 


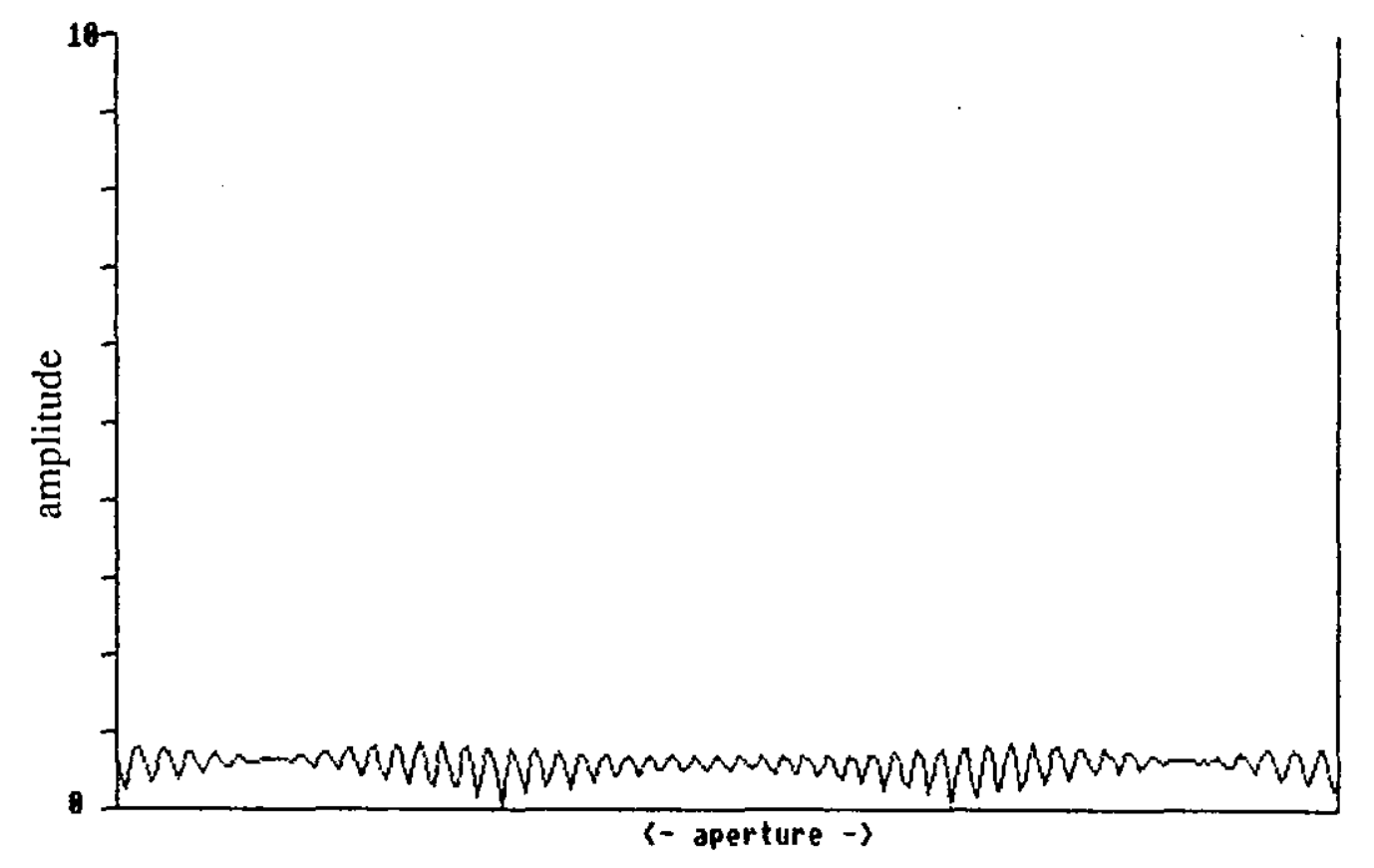

Eig. 4.14(e) Reconstructed Targets with a Biased Transducer Error Motion of Amplitude $0.5 \lambda$ and a Frequency of 1 cycles/aperture.

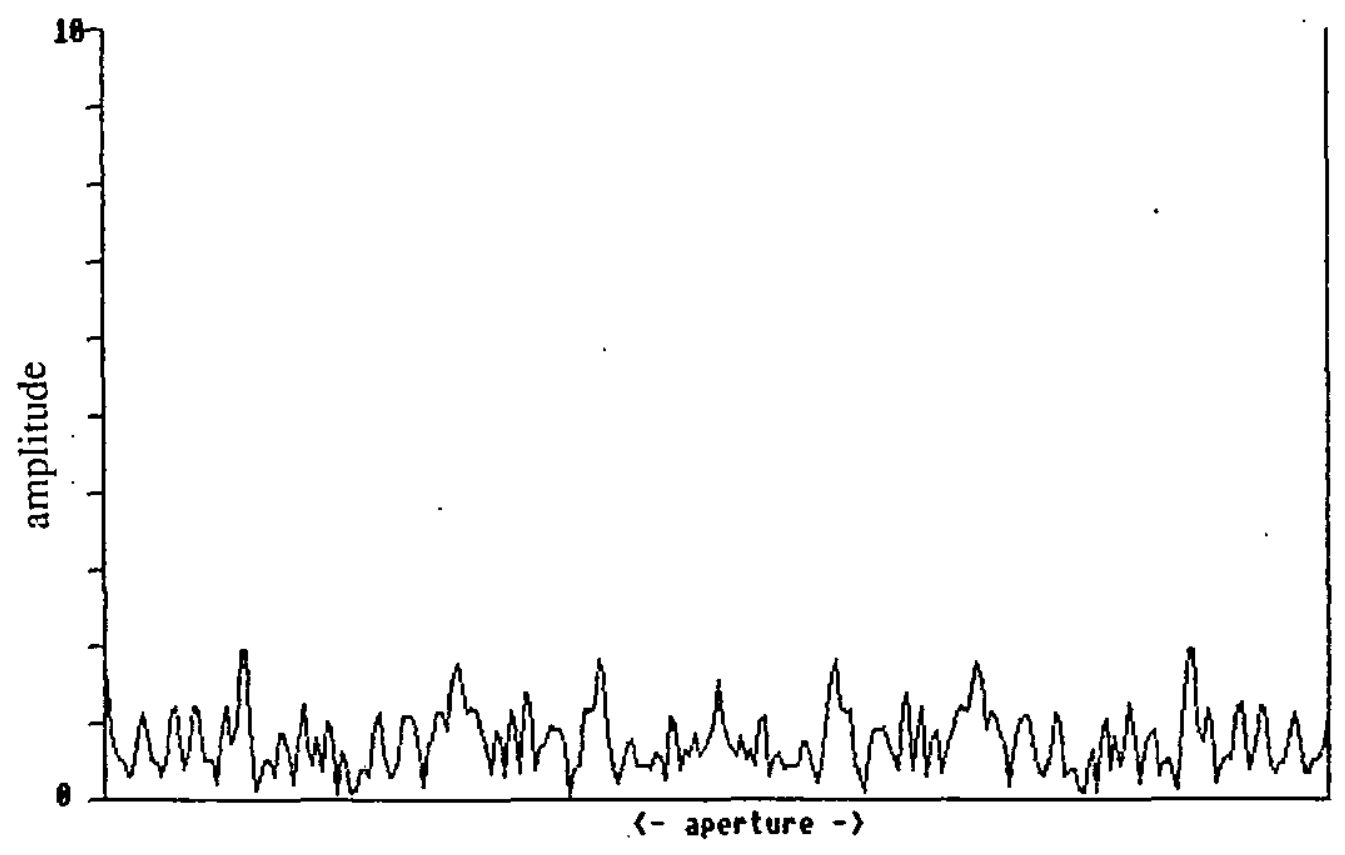

Fig. 4.14(f) Reconstructed Targets with a Biased Transducer Error Motion of Amplitude $0.5 \lambda$ and a Erequency of 5 cycles/aperture. 


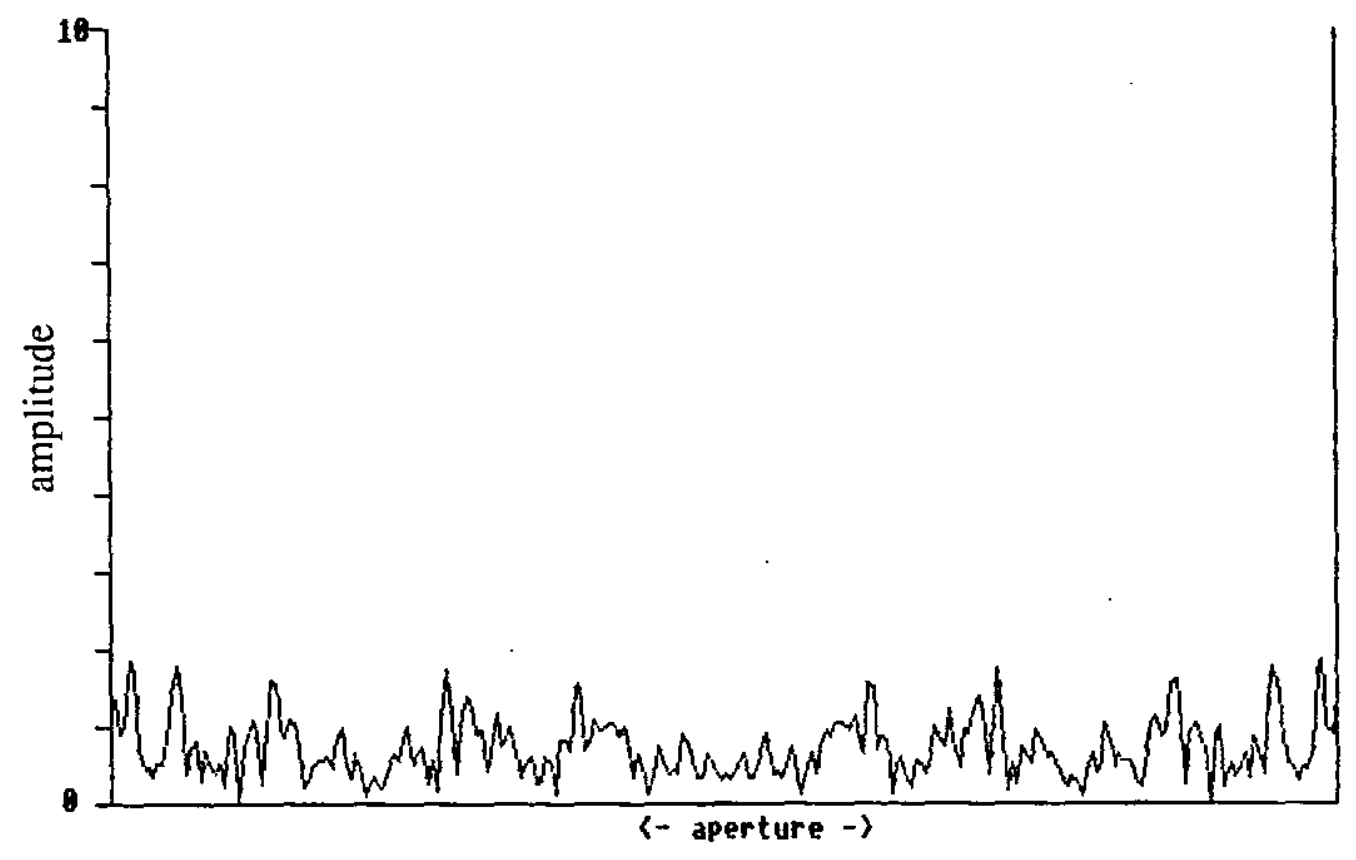

Fig, 4.14(g) Reconstructed Targets with a Biased Transducer Error Motion of Amplitude $0.5 \lambda$ and a Frequency of 50 cycles/aperture. 


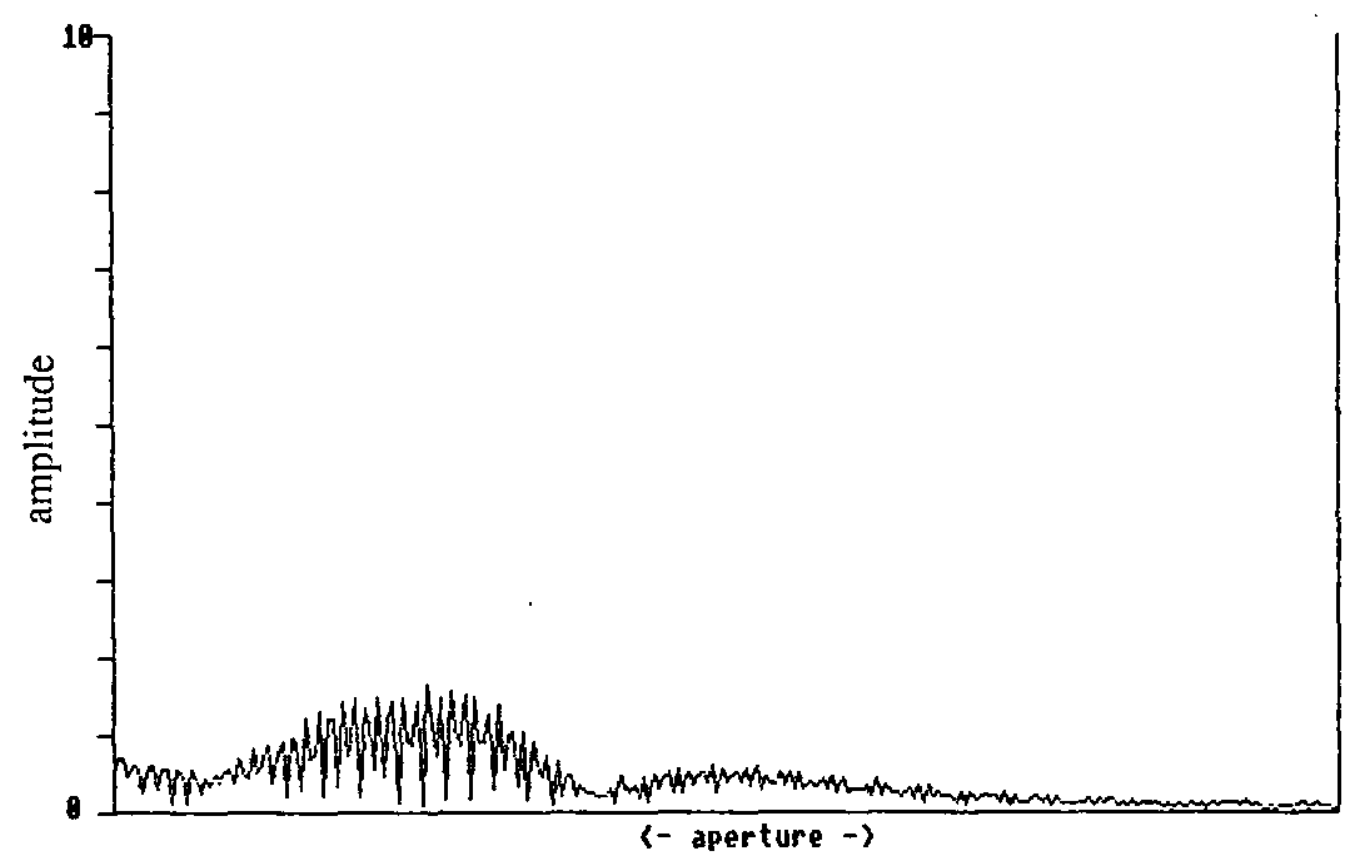

Fig, 4.15(a) Reconstructed Targets with a Biased Transducer Error Motion of Amplitude $1 \lambda$ and a Frequency of 0.5 cycles/aperture.

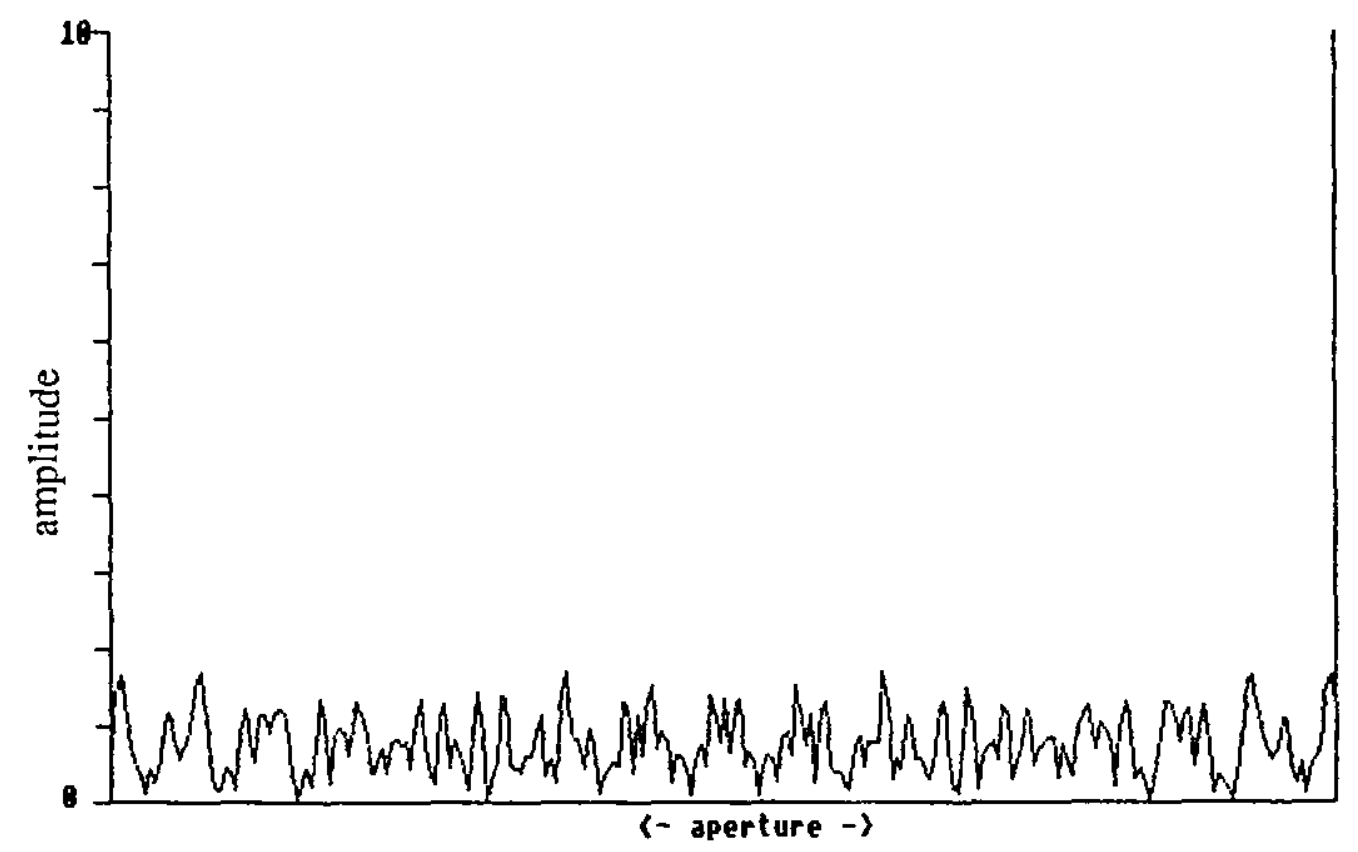

Eig, 4.15(b) Reconstructed Targets with a Biased Transducer Error.Motion of Amplitude $1 \lambda$ and a Frequency of 1 cycles/aperture. 


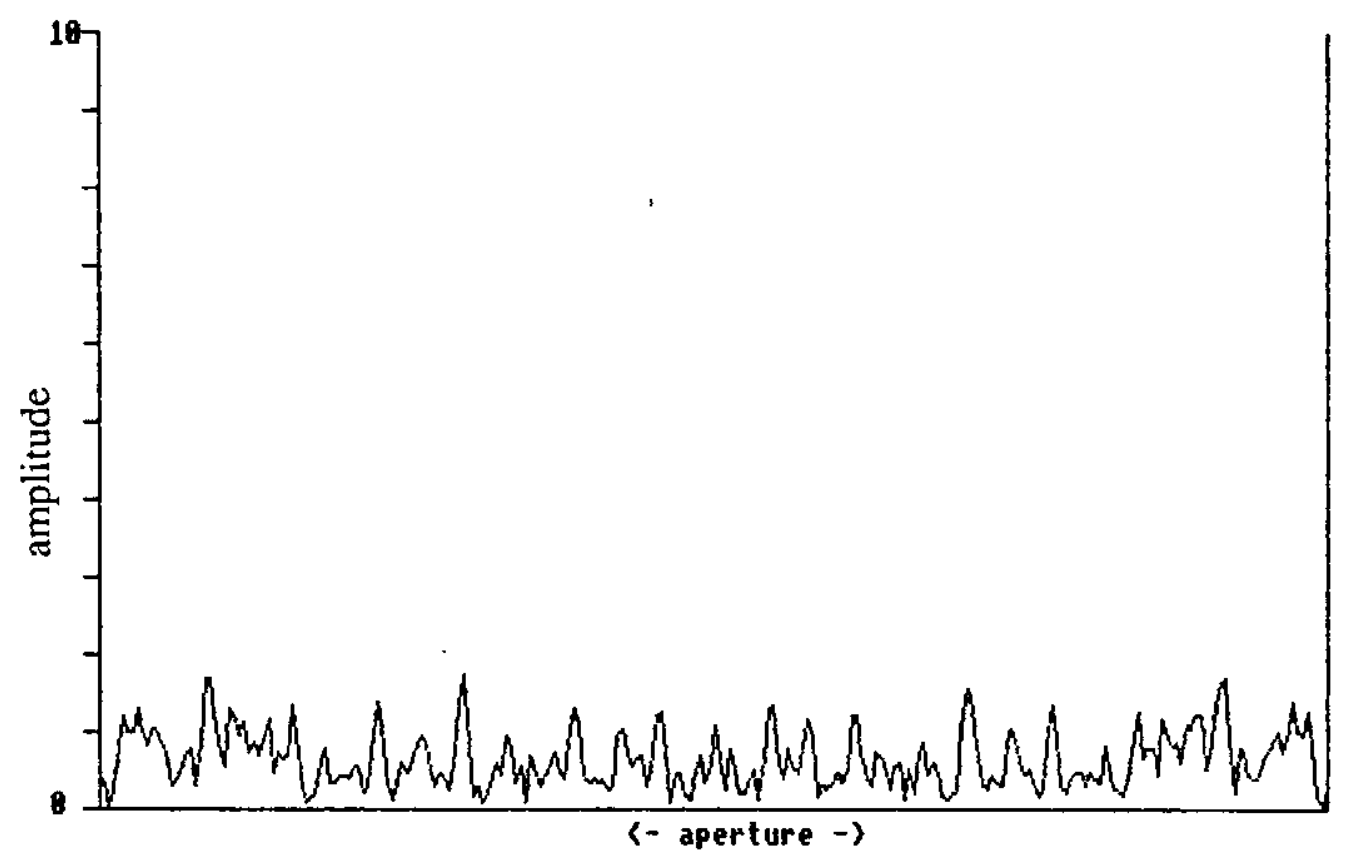

Fig. 4.15(c) Reconstructed Targets with a Biased Transducer Error Motion of Amplitude $1 \lambda$ and a Frequency of 5 cycles/aperture.

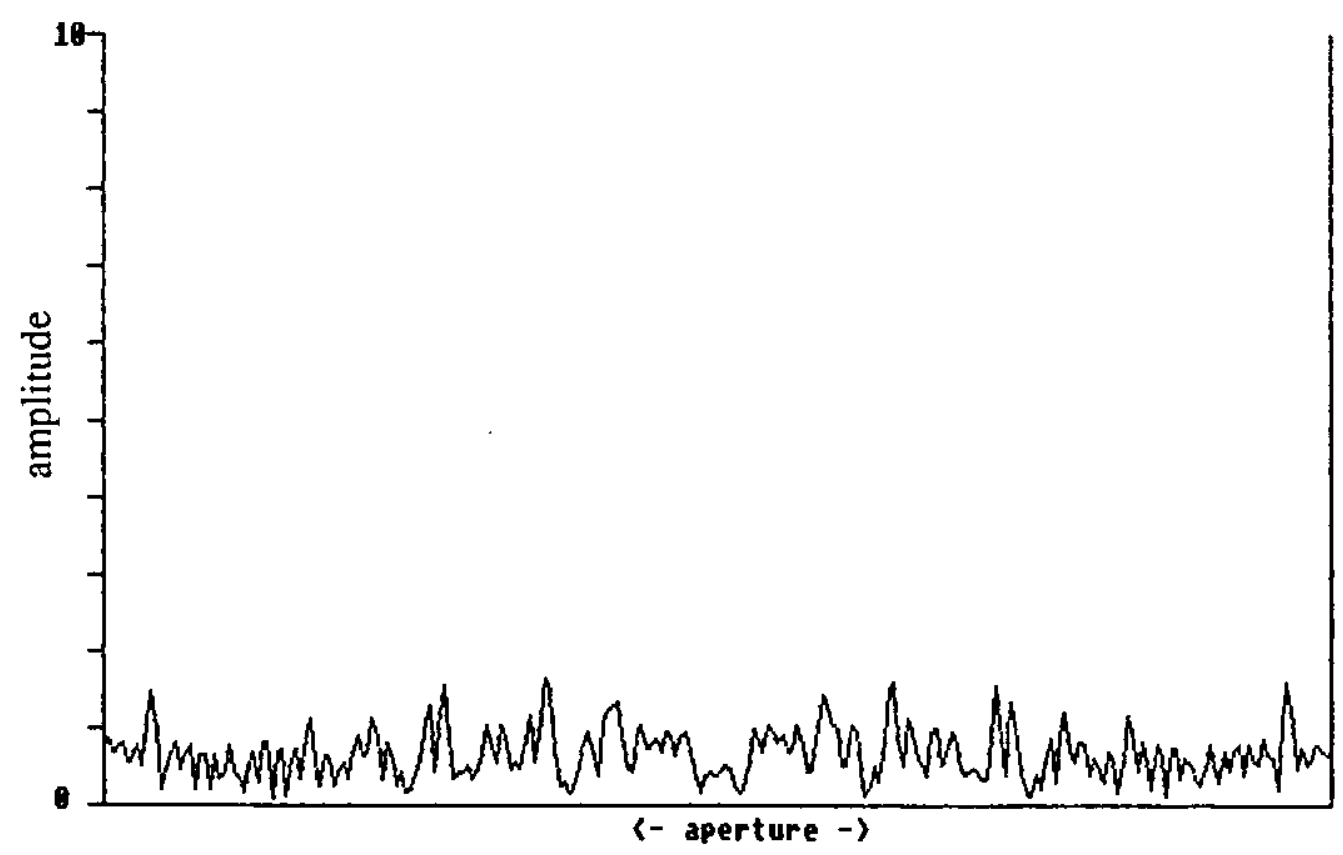

Fig. 4.15(d) Reconstructed Targets with a Biased Transducer Error Motion of Amplitude $1 \lambda$ and a Frequency of 50 cycles/aperture. 
synthetic aperture system are prone to degradation even when the error motion is very small. The degradation effects can be categorized as [41];

(a) Target translation.

(b) Target "width" altered.

(c) Spurious target generation.

(d) Increase in the side lobe structure.

The degradation is small when the frequency of the biased error motion is less than $0.5 \lambda$ cycles/aperture but the degradation increases with an increases in error motion amplitude. At lower amplitude levels there is reduction in the degradation as the frequency of the error motion is increased.

\subsection{Transducer Error Motion Correction}

Two reference targets at known positions, are used to correct the error in phase of the received signal due to unknown transducer motion. The position of the transducer, $P(x, y)$, can be ascertained by the knowledge of the distances between the point $P$ and each of the reference targets as shown in Fig. 4.16. The distances are calculated from the echo returns from each of the reference targets, and the position of $P$ is determined by solving simultaneously equations [4.15] for two intersecting arcs.

$$
\begin{aligned}
& (x-x 1)^{2}+(y-y 1)^{2}=z 1^{2} \\
& (x-x 2)^{2}+\left(y-y^{2}\right)^{2}=z^{2}
\end{aligned}
$$

where $z 1$ and $z 2$ are the distance between the transducer and reference target $T 1 \quad\left(\times 1, y_{1}\right)$ and $T 2$ $\left(x_{2}, y^{2}\right)$ respectively. 
The general solution to equation $[4.15]$ is

$$
\begin{aligned}
& x=b \pm \sqrt{\frac{\left[b^{2}+a\left(x 1^{2}+y 1^{2}+c 1^{2}-2 y 1 c 1-z 1^{2}\right)\right]}{a}}[4.16] \\
& y=c 1-c 2 x
\end{aligned}
$$

where

$$
\begin{aligned}
a & =1+c 2^{2} \\
b & =x 1+c 1 c 2-y 1 c 2 \\
c 1 & =\frac{z 1^{2}-z 2^{2}-y^{2}+y 2^{2}-x 1^{2}+x 2^{2}}{2\left(y^{2}-y^{1}\right)} \\
c 2 & =\frac{x 2-x 1}{y^{2}-y^{1}}
\end{aligned}
$$

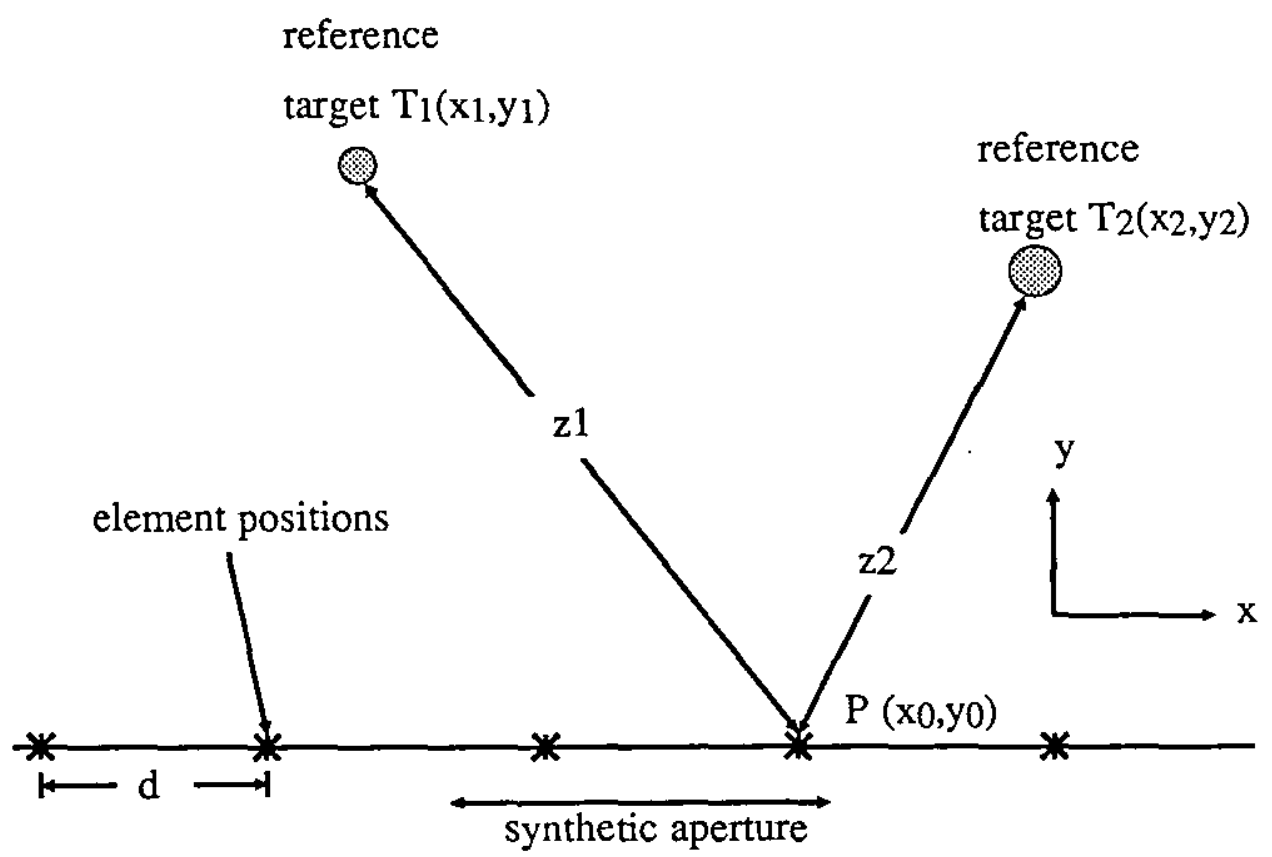

Fig 4.16.Reference Target Geometry, 
F1g. 4.17 and F1g. 4.18 shows the effects of applying the above method to the data simulated for the various error motions. The targets have been successfully reconstructed for all the situations. Fig. 4.17(a) and Fig. 4.17(b) illustrate the effects of applying this technique to the data recelved when there $1 s$ a random transducer error motion of amplitude $1 \lambda$ and $2 \lambda$ respectively. Fig. 4.18 is the resulting image when this technique 1s applied to the data recelved when there $1 \mathrm{~s}$ a biased transducer error motion of amplitude $\emptyset .5 \lambda$ and frequency 5 cycles/ aperture (Fig. $4.18(\mathrm{a})$ ) and 50 cycles/aperture (Fig. $4.18(b)$ ) and of amplitude $5 \lambda$ and frequency 5 cycles/ aperture (F1g. $4.18(\mathrm{C})$ ) and 50 cycles/aperture (F1g. $4.18(d))$.

Ir all the considered situations the targets have been successfuliy reconstructed with the expected level of sidelobes and spurious targets have not been generated. F1g. 4.19 is a flowchart of the complete lmage reconstruction piocess using reference targets to compensate for transducer motion errors.

In an ocean deployed system, the reference targets are only required at the start of the operation and as strong targets come into the image these in turn are useo as the 'reference' targets to reduce the effects of transducer position uncertainty. 


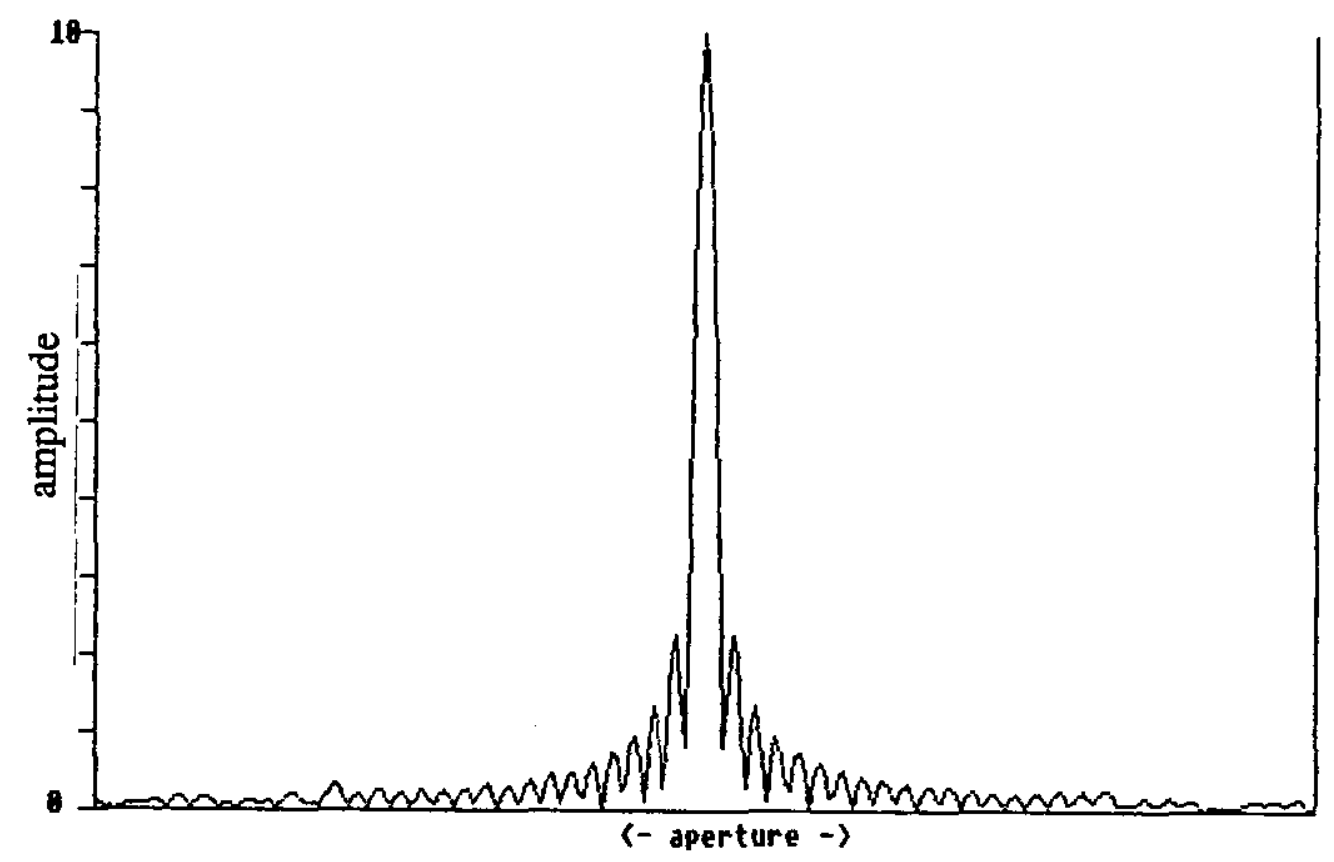

Fig, 4.17(a) Reconstructed Image when Reference Targets are used to Reduce the effects of Random Error Motion of $1 \lambda$ Amplitude.

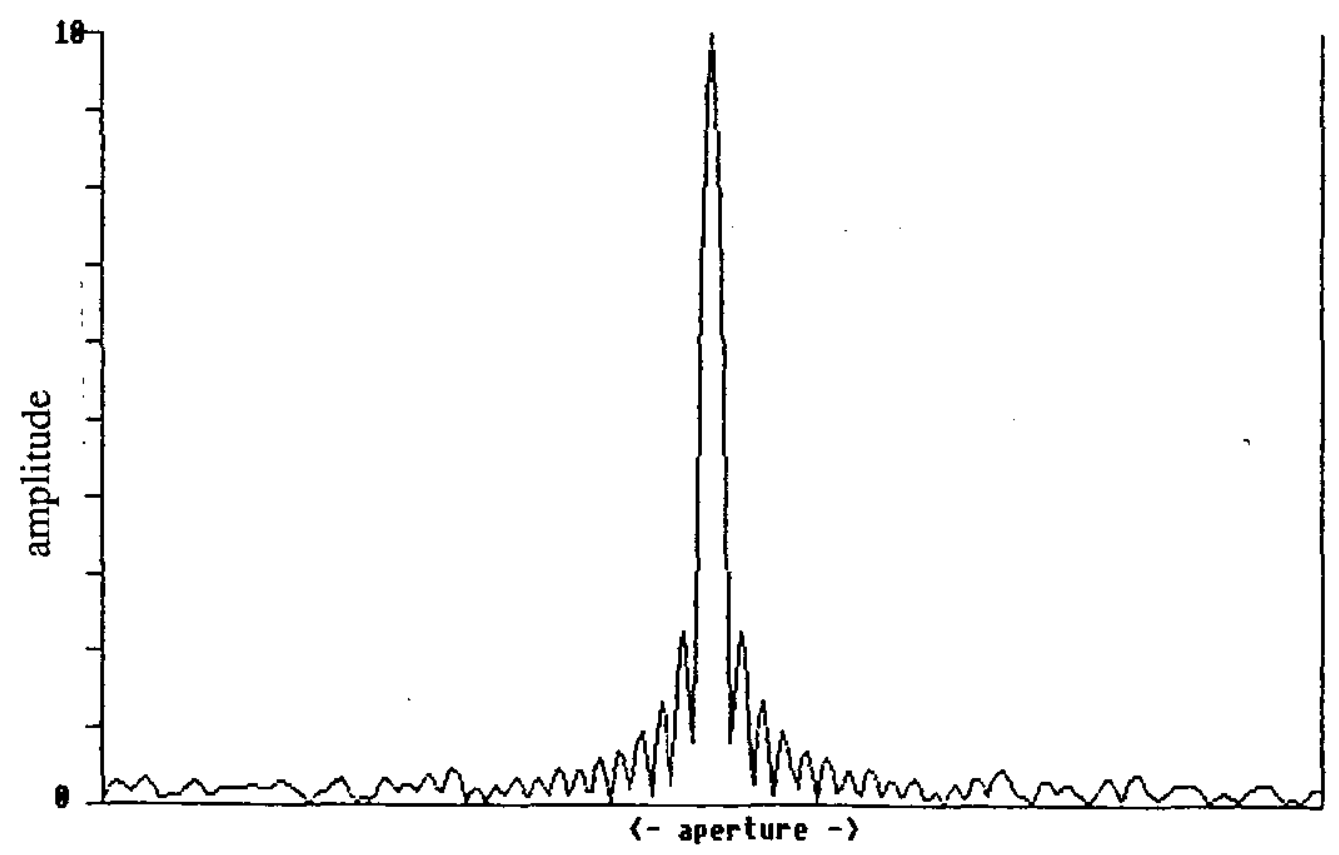

Fig. 4.17(b) Reconstructed Image when Reference Targets are used to Reduce the effects of Random Error Motion of $2 \lambda$ Amplitude. 


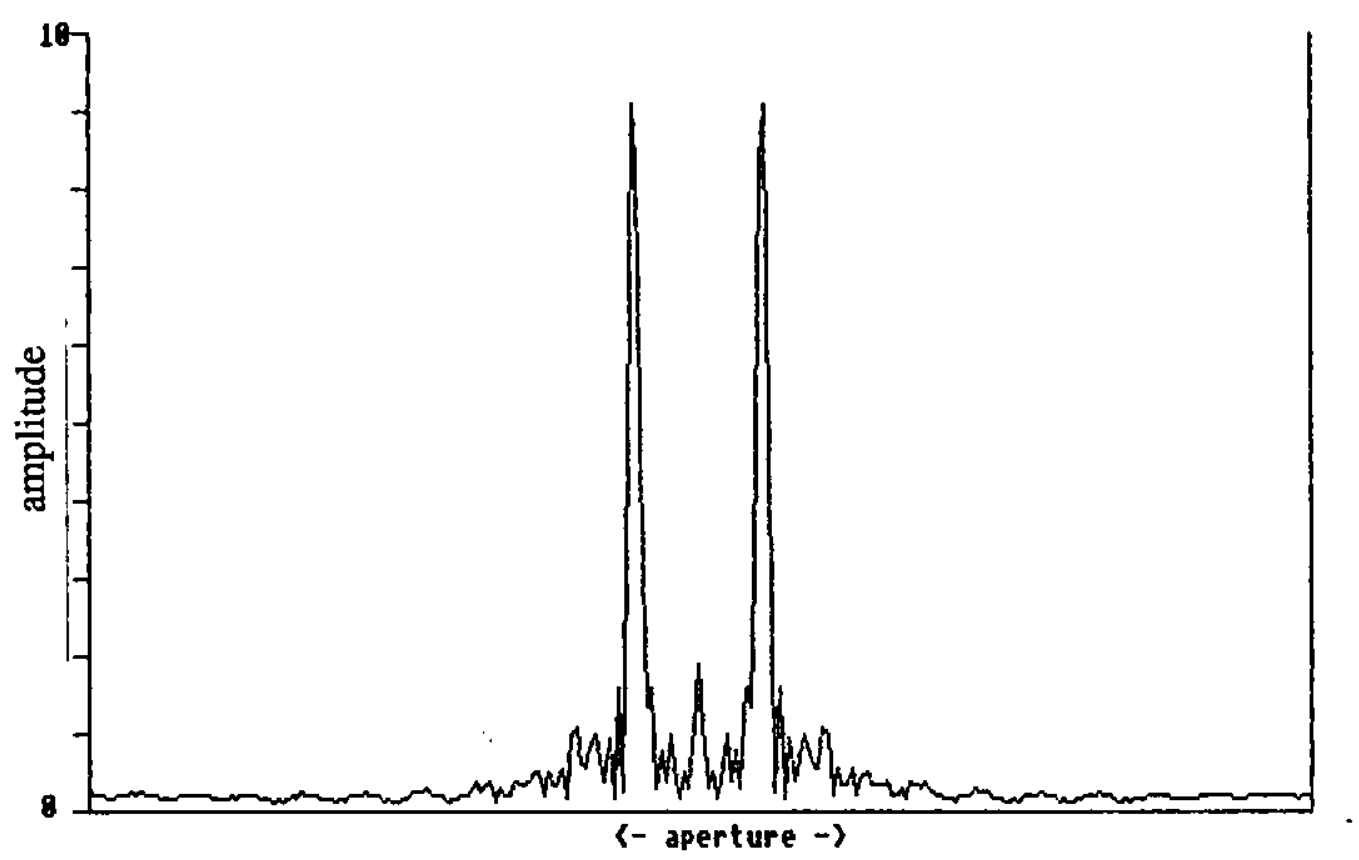

Eig. 4.18 (a) Reconstructed Image when Reference Targets are used to Reduce the effects of Biased Error Motion of Amplitude $0.5 \lambda$ and a Frequency of 5 cycles/aperture.

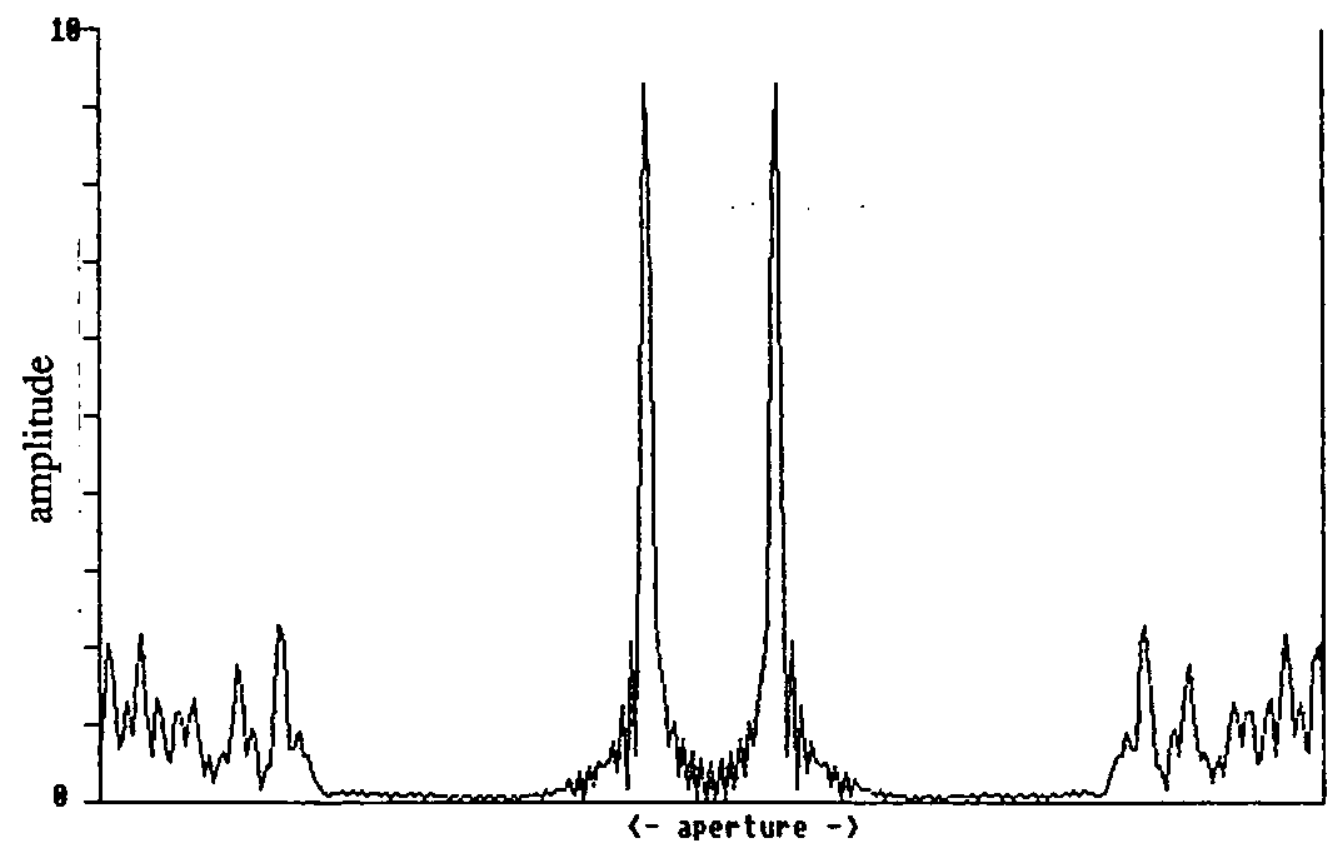

Fig, 4.18 (b) Reconstructed Image when Reference Targets are used to Reduce the effects of Biased Error Motion of Amplitude $0.5 \lambda$ and a Frequency of 50 cycles/aperture. 


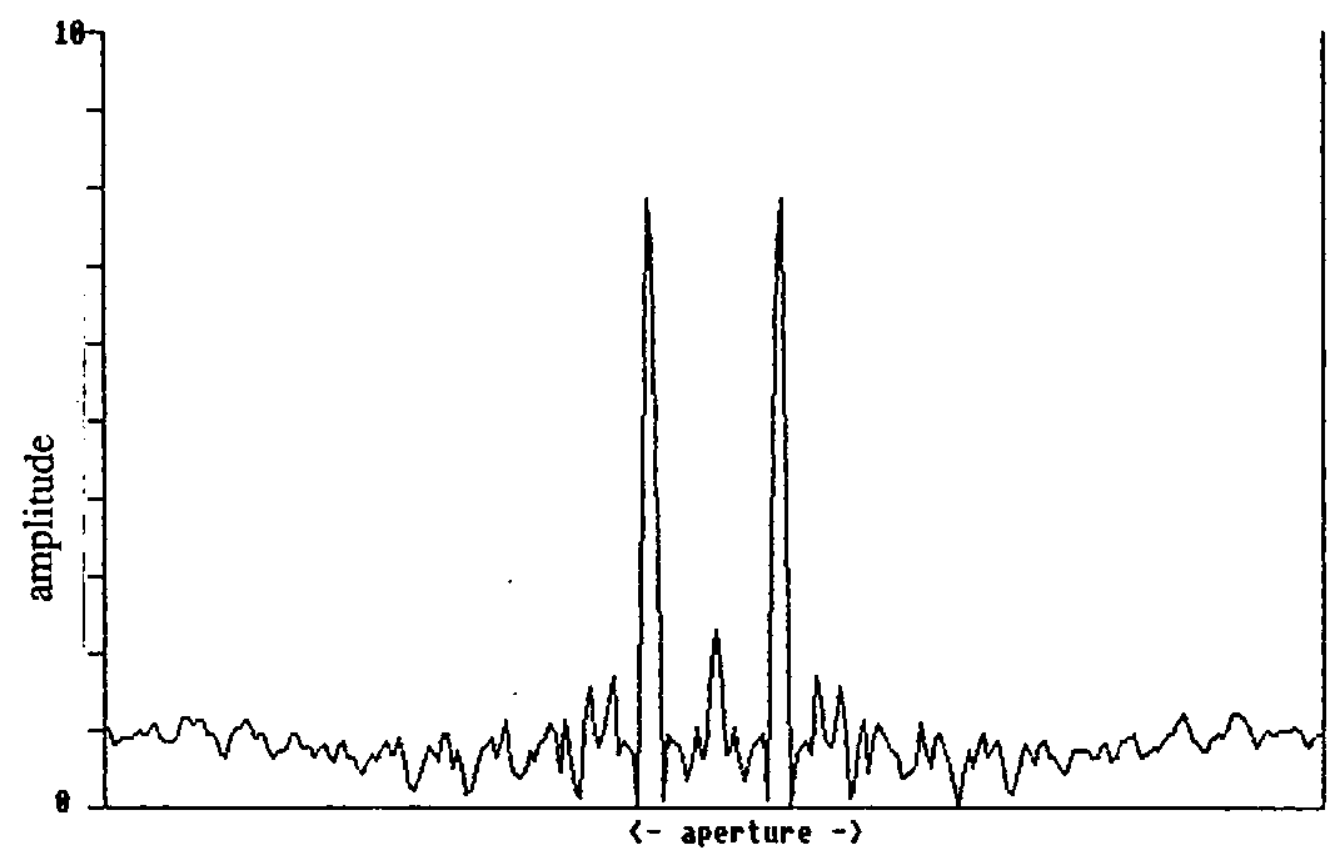

Fig. 4.18 (c) Reconstructed Image when Reference Targets are used to Reduce the effects of Biased Error Motion of Amplitude $1 \lambda$ and a Frequency of 5 cycles/aperture.

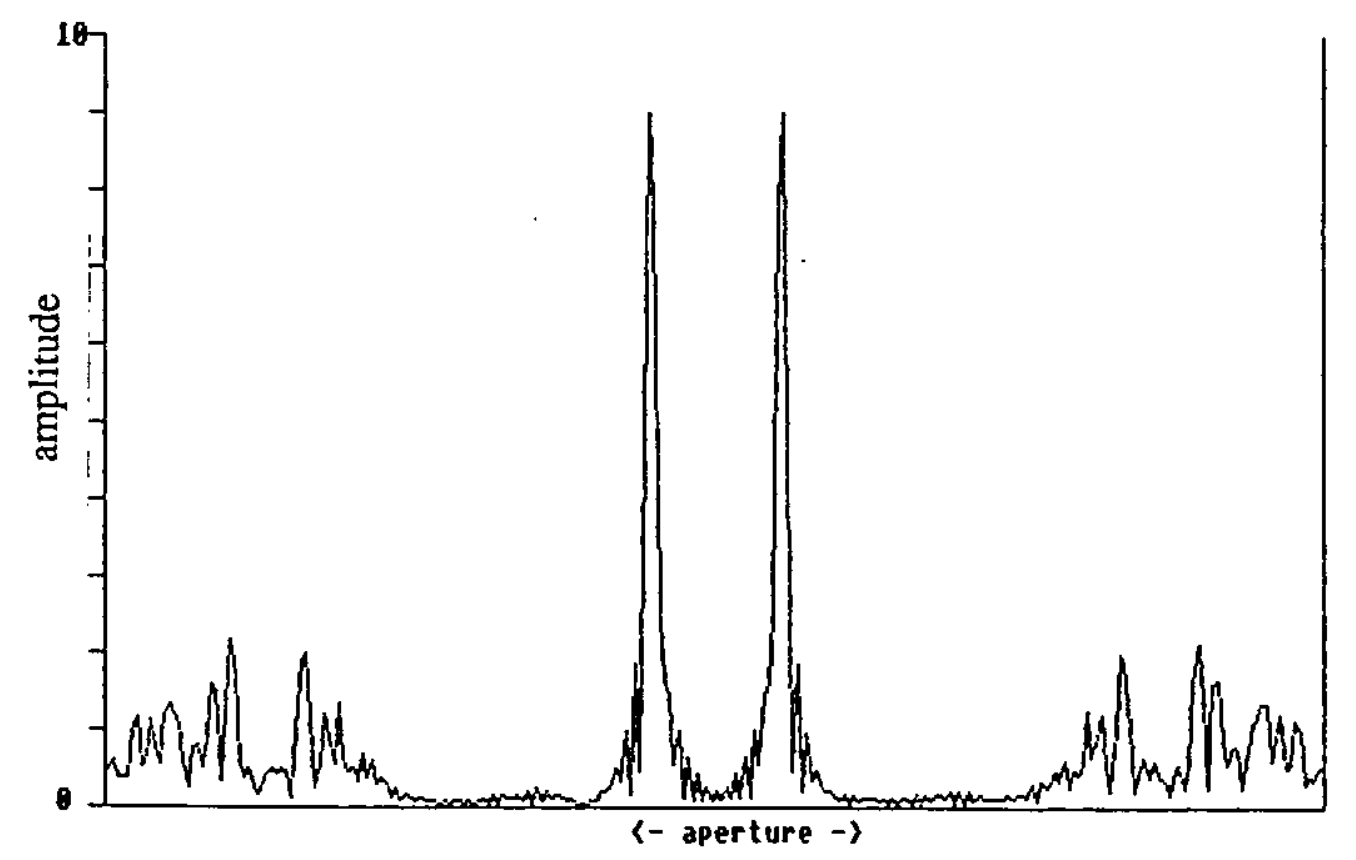

Eig. 4.18 (d) Reconstructed Image when Reference Targets are used to Reduce the effects of Biased Error Motion of Amplitude $1 \lambda$ and a Frequency of 50 cycles/aperture. 


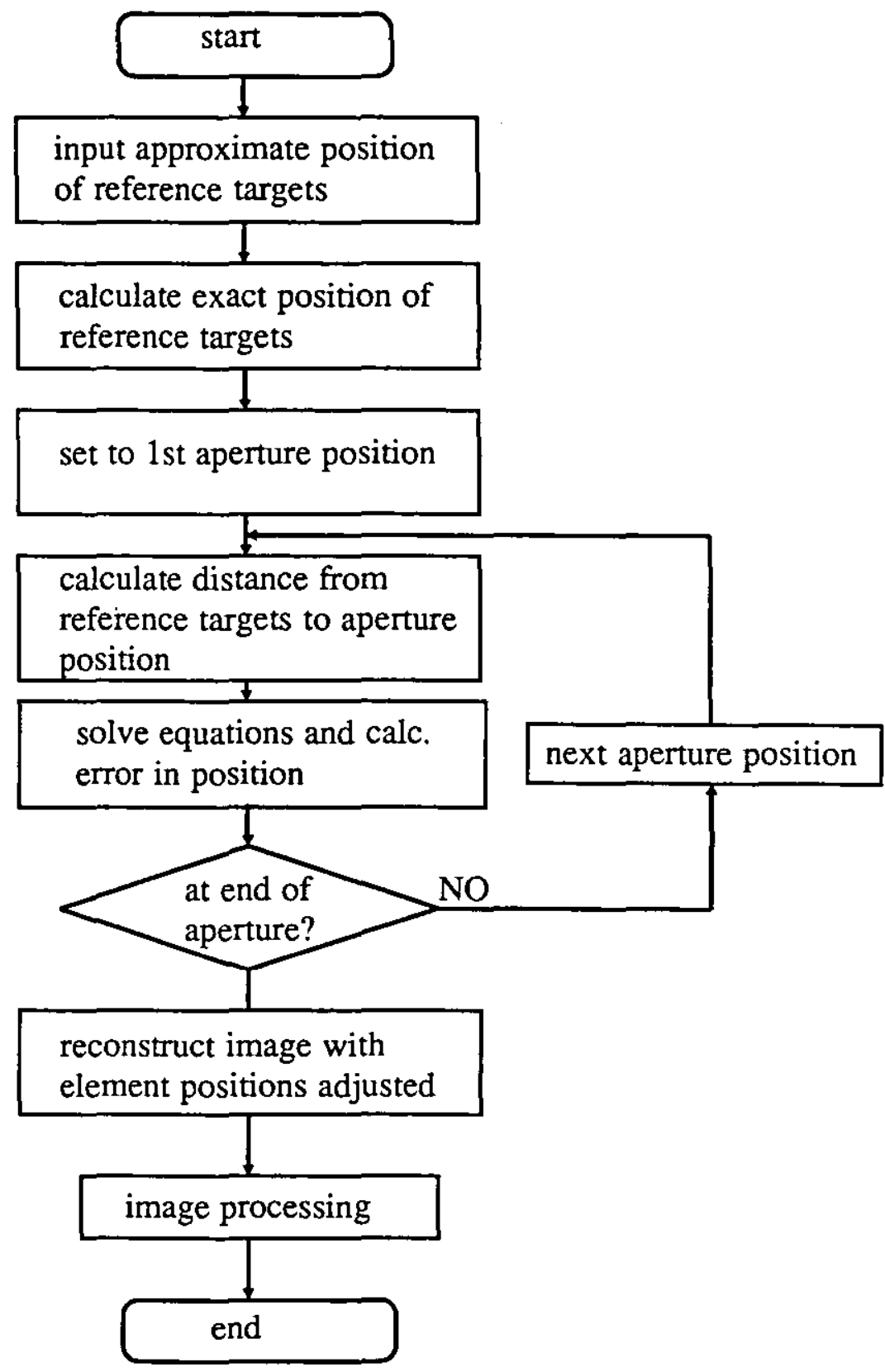

Fig. 4.19 Image Reconstruction with Transducer Error Motion Correction 


\section{IMAGE PROCESSING}

\section{1 Introduction}

The output of a sonar system is often a two dimensional image on a monitor. Target motion. inhomogeneities in the water such as temperature. pressure and salinity gradients and unknown movements in the transducer path can lead to a loss of coherency of the recelved signal and hence a degraded reconstructed image from a synthetic aperture sonar system. The degradation can take one or more of the following forms:
target blur and smear.
(ii) spurious targets generated.
(iii) target size altered.
(iv) reduction in target strength.
(v) target displacement.

In this chapter the use of image processing techniques to reduce degradations of sonar images will be considered but first some baslc image processing concepts ore introduced.

Image processing methods are directed towards one of three goals: the improvement of pictorial information for human interpretation or subjective appraisal, the processing of image data for autonomous machine recognition or the processing of images for optimum transmission over a link. 
Image processing techniques can be subdivided into rectificotion, enhancement, restoration and reconstruction.

Image rectification is concerned with spatial transformations that can remove geometric distortion or permit images to be properly reglstered with respect to each other.

Image enhancement is concerned with improved presentation (e.g. by noise reduction, compensation for non-linearities of the recording medium, contrast adjustment and edge sharpening) of images whose essential information is clouded but is nevertheless visually apparent before processing.

Image restoration involves estimating the parameters of the distortion. which are not known a priori and using them to reduce the degradation.

Image reconstruction is concerned with recovering detail in severly blurred images, the causes of whose imperfections are known a priori.

The linear technique of image enhancement and the non-linear technique of image restoration will be applied to typical images obtained using synthetic aperture systems. But first image processing terminolagy and some basic concepts are discussed.

Detailed analysis of the subject can be found in the following references: Rosenfeld [82]. Gonzalez and Wintz [83] and Pratt [84].

\section{2 Cont inuous Image Model}

A simple representation of a physical monochrome image is given by a real-valued, two dimensional light intensity function, denoted by $f(a, b)$. where the value of $f$ at spatial co-ordinates $(a, b)$ of an infinite plane $(A, B)$ 
represents the intensity (or brightness) of the image at that point.

Since the amount of 1 ight reaching an observer from a physical image is finite and non-negative: $0 \leqslant f(a, b) \leqslant \infty$. It is also reasonable to consider a lower and upper bound $K_{\min }$ and $K_{\max }$ to the possible brightness of any physical picture; thus for an image function $f$ :

$$
K_{\min } \leqslant f(a, b) \leqslant K_{\max } \text { for all }(a, b) \text {. }
$$

The interval $\left[K_{\min } \cdot K_{\max }\right]$ is called the grey scale, but conventionally this scale is shifted to the interval [O.K] and $\theta$ represents black and $K$ represents peak white. Then $0 \leqslant f(a, b) \leqslant K$. A real image is limited in extent by physical characteristics of the lmaging system or the recording medium. The image function $f(a, b) \geqslant 0$ only in some bounded region.

\section{3 Digital Image Representation}

In order to process an Image by digital computer, the function $f(a, b)$ must be digitised both spatially and in amplitude. Digitisotion of the spatial co-ordinates $(a, b)$ is referred to as image sampling and amplitude digitisation is called grey level quantisation. A digitised image may be thought of as a discrete, two dimensional array of real numbers; i.e.

$$
f(x, y)=\left[\begin{array}{ccccc}
f(0,0), & f(0,1), & f(0,2), & \ldots & f(0, N-1) \\
f(1,0), & f(1,1), & f(1,2), & \ldots & f(1, N-1) \\
\ldots & \ldots & \ldots & \ldots & \ldots \\
\ldots & \ldots & \ldots & \ldots & \ldots \\
f(M-2,0), f(M-2,1), f(M-2,2) & \ldots f(M-2, N-1) \\
f(M-1,0), f(M-1,1), f(M-1,2) & \ldots f(M-1, N-1)
\end{array}\right]
$$

The members of such an array are referred to as image elements or pixels. 
Consider an $M$ by $N$ array of sampled points quant ised to a range of $K$ discrete levels. The digital image function may then be represented by $M \times N \times \log _{2} K$ bits of information. It is desirable to select the minimum values of these parameters. whilst retaining all information present in the original continuous image.

It is common practice in digital image processing to let these quantities be integer powers of 2 , that is

$$
M=2^{m} \text {. } \quad N=2^{n} \text {. and } K=2^{9} \text {. }
$$

Also the array is normally square and hence $M=N$.

\subsection{The Two-Dimensional Filter}

With the image in a sampled and quantised form. We may consider the operations on it by a digltal computer. But first we shall briefly consider the two dimensional fourier transform and convolution.

The two dimensional discrete form of the fourler transform is given by the following equations.

$$
\begin{aligned}
& F(u, v)=\frac{1}{N} \sum_{x=0}^{N-1} \sum_{y=0}^{N-1} f(x, y) \exp \{-j 2 \pi(u x, v y) / N\} \quad[5.1] \\
& f(x, y)=\frac{1}{N} \sum_{u=0}^{N-1} \sum_{v=0}^{N-1} f(u, v) \exp \{j 2 \pi(u x, v y) / N\} \quad[5.2]
\end{aligned}
$$

The two dimensional transform may be computed as sequential row and column one dimensional transforms. Evaluation of the discrete fourler transform by this method requires $N^{3}$ multiplications and additions and can consume. large amounts of computer time when $N$ is large. A more efficlent way of calculating the transform is by using the 
fast fourier transform (FFT) [85]. and for images of 128 by 128 pixels and above there is a significant saving in computation time by using the FFT method.

A linear (or enhancement) operation on an image $f(x, y)$ may be represented by the convolution of the point spread function (PSF) of the operator $h(x, y)$ and the image itseif $[74]$. The resulting image $r(x, y)$ is given by

$$
r(x, y)=h(x, y) \odot f(x, y)
$$

where $\mathcal{O}$ denotes convolution.

This is also equivalent to

$$
R(u, v)=H(u, v) F(u, v)
$$

Thus to carry out the required enhancement we can either convolve a particular PSF with the image or we can multiply the image spectrum with an appropriate transfer function $H(u, v)$.

Digital convolution is similar to continuous convolution except that the integral is replaced by a summation and may be carried out as follows:

The products of the image pixel brightness values. covered by the convolving array $h(x, y)$ and the convolving array entries are taken and summed to give the response. This response is then used to define a new brightness value for the pixel currently at the centre of the convolving array. When this is done for every pixel in the image a modified image is produced that enhances or smoothes geometric features according to the specific numbers in $h(x, y)$.

A three by three convolving array is illustrated in Fig. 5.1 and the response for image pixel $x, y$ is 


$$
r(x, y)=\sum_{i=-1}^{+1} \sum_{j=-1}^{+1} f(x-1, y-j) h(i, j)
$$

where $h(i, j)$ is the convolving mask entry at that location.

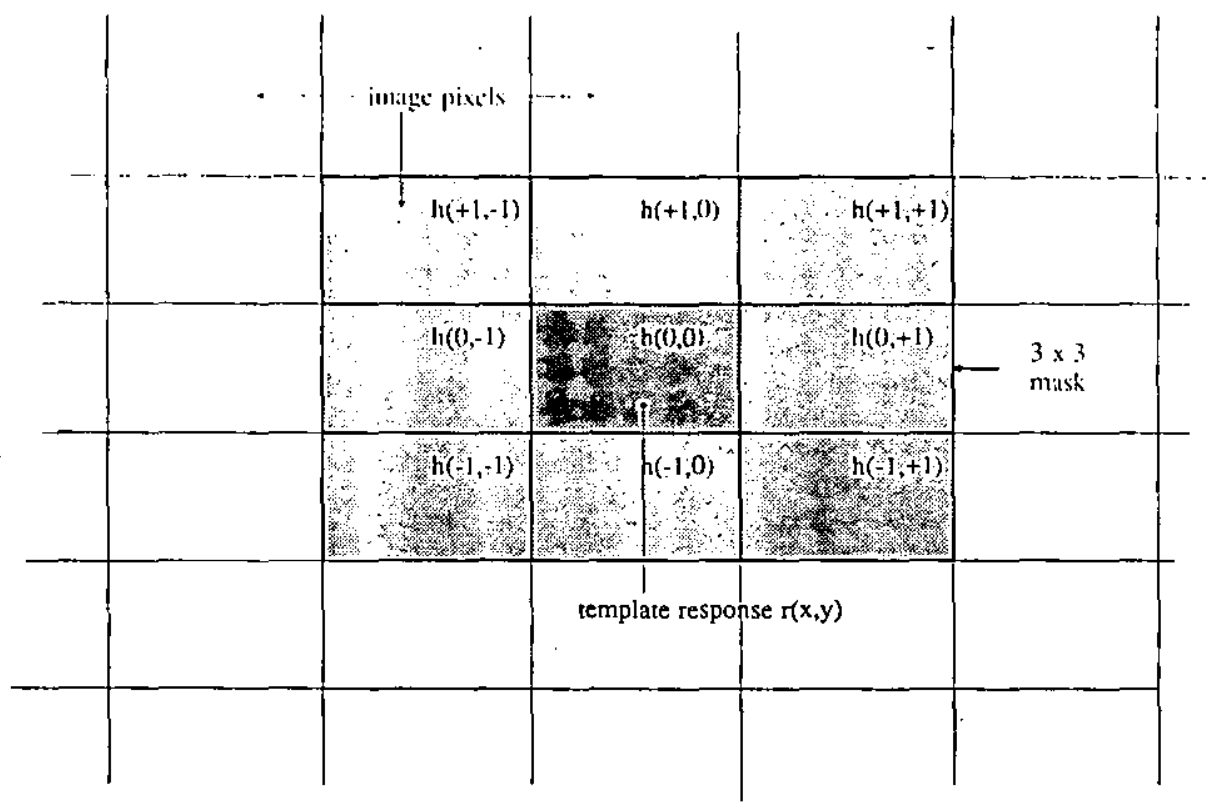

Fig.5.1 A 3 by 3 Convolving Array.

\subsection{Image Enhancement}

There are a number of almost standard techniques that have become associated with enhancement operations on images. These include grey scale modification, histogram equalization, edge sharpening and smoothing.

\subsubsection{Smoothing}

\subsubsection{Simple Nelghbourhood Average}

Position invariant, neighbourhood averaging methods 
are based upon the generally high degree of correlation between an image pixel and its neighbours. Atyplcal pixels representing nolse components have a higher spatial frequency spectrum than pixels representing the original image. Spatial smoothing may consequently be thought of as a simple low pass filtering operation and is anologous to frequency domain filtering.

Noise smoothing for "snow" removal in TV images was one of the first applications considered for digital image processing [79]. Noise produces on overall granular effect which can hide the information we want to see. Consider a simple image of a uniform intensity area on a similarly uniform background. Fig. 5.2 shows a line of pixels and their brightness values taken across the image. We can simulate the effect of additive noise by generating a noise fleld and pointwise adding it to the image. As a result of adding the noise field the simple image is now degraded to the form shown in Fig. 5.3. If the noise component is not too large then an averaging operation can be expected to reduce its visual effect. The average is taken over a small neighbourhood around the current image point. Typlcally each point will be surrounded by eight other points and the centre point is replaced by the average of these. Fig. 5.4 illustrates typical weights used in the convolving mask. The result of applying a simple moving average filter shown in Fig. $5.4(a)$ to the test image is shown in Fig. 5.5. The noise has certainly been reduced but the edges of the block are no longer as sharp and blur has been introduced.

\subsubsection{Smoothing with a Threshold}

Spatial smoothing algorithms, if applied indiscrimin -antiy. may introduce blur. especially if a large 


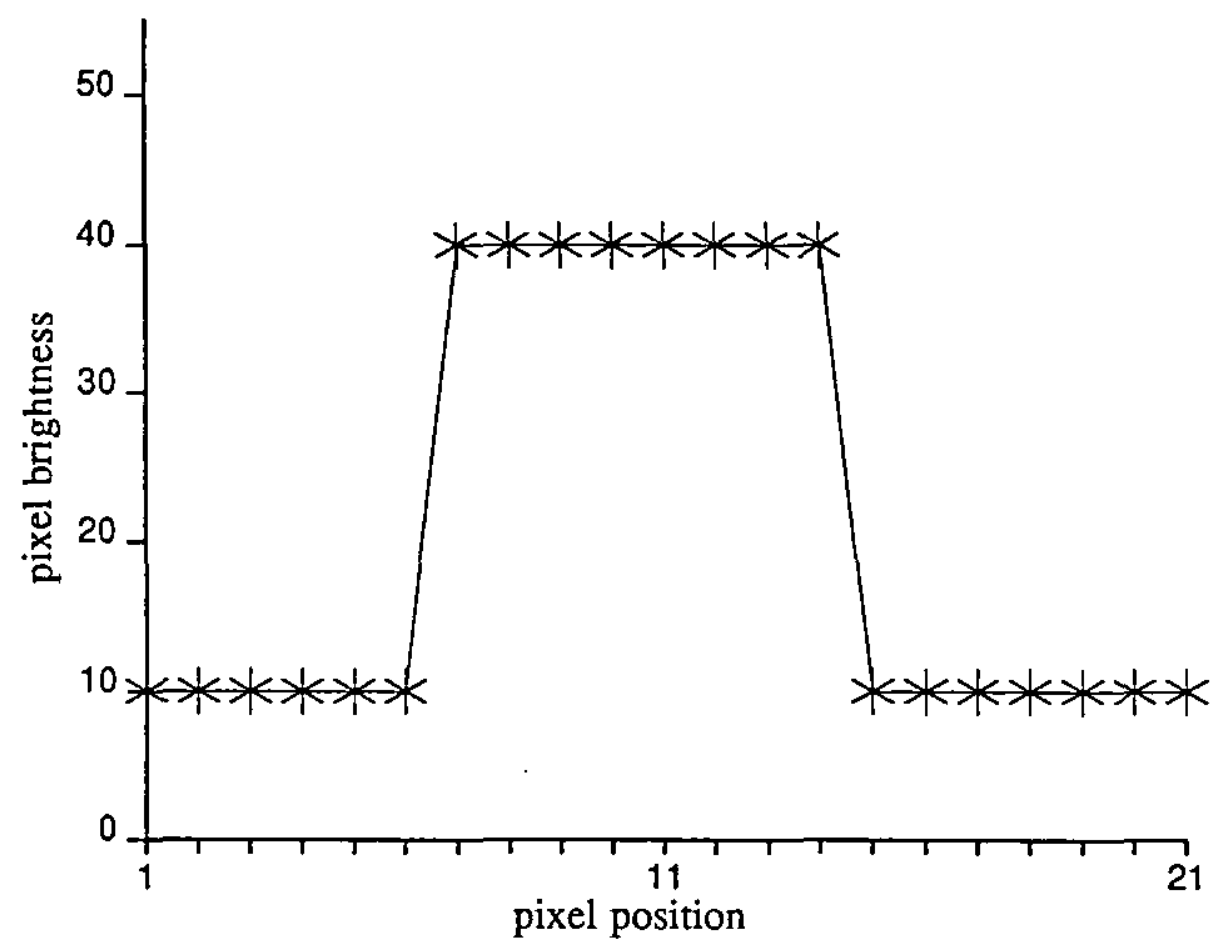

Fig. 5.2 A Line of Pixels taken across a Simple Image

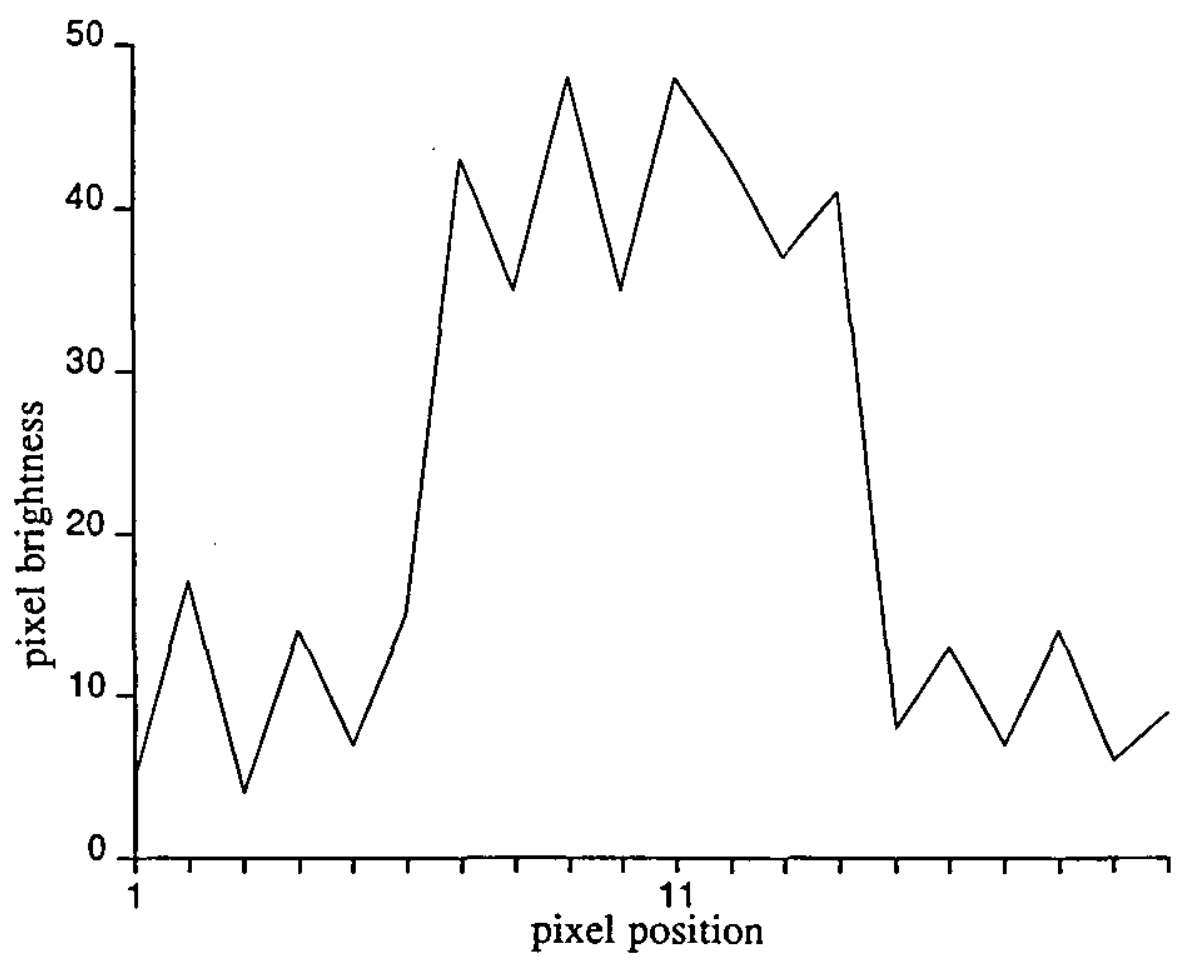

Fig, 5.3 Image with simple additive noise 


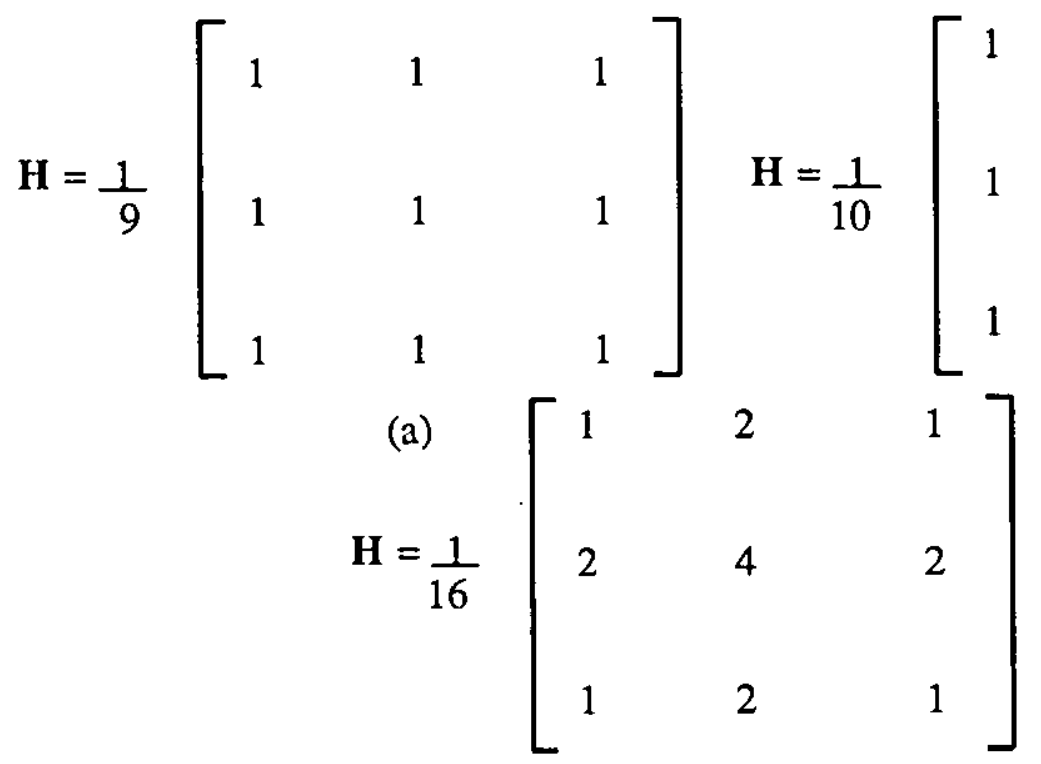

(c)

Fig. 5.4_Some Typical Smoothing Convolution Masks.

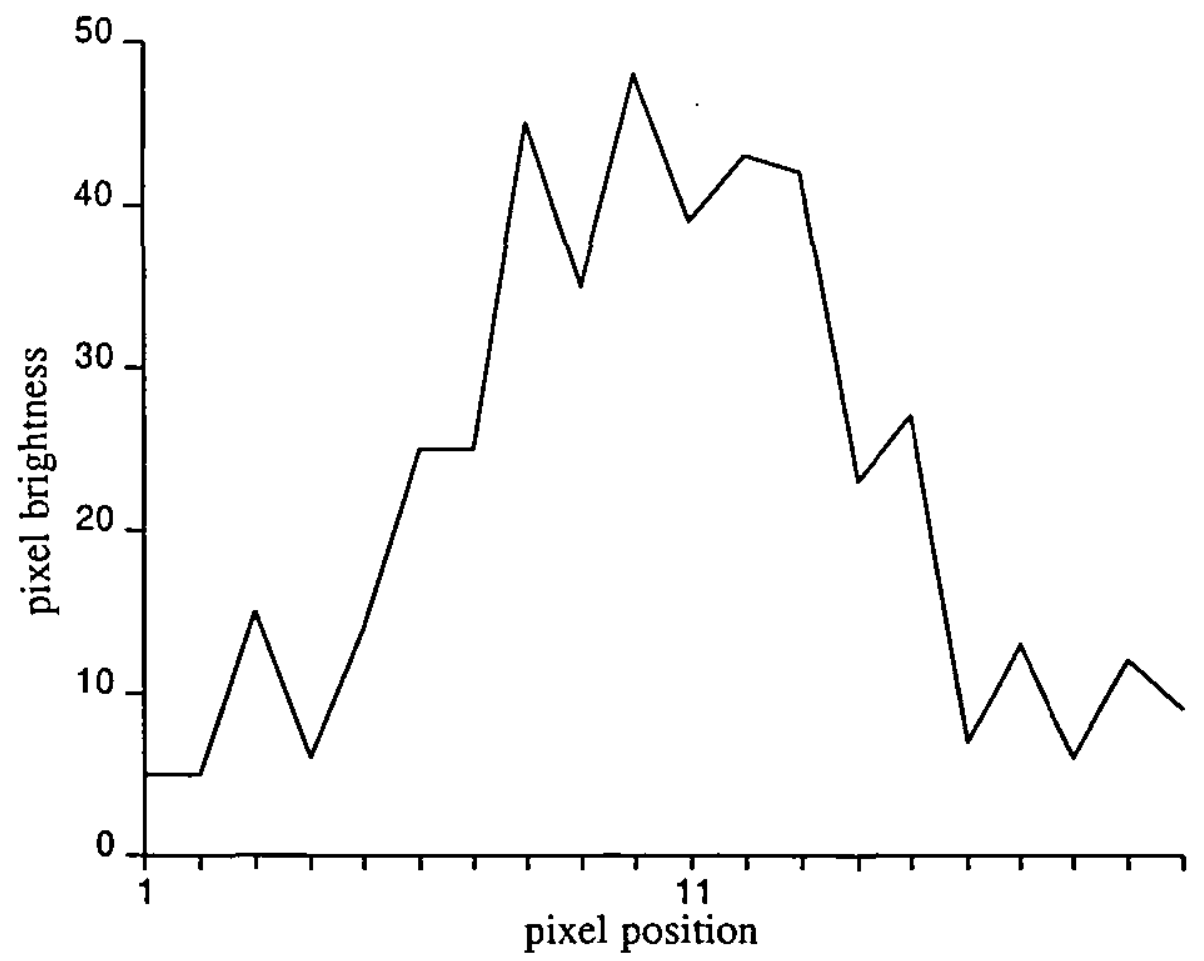

Fig, 5.5 Simple Smoothing ofa 'Noisy' Image. 
neighbourhood is considered. It is desirable to permit smoothing of regions of high average correlation without introducing unwanted blurring of sharp edges. Non I l near methods may be used to reduce this loss of detail around the edges, for example. if a threshold is used.

Let

then

$$
\rho(x, y)=\frac{1}{N^{2}} \sum_{x, y=0}^{N-1} f(x, y)
$$

$$
\begin{aligned}
r(x, y) & =p(x, y) \text { if }|f(x, y)-p(x, y)|<T \\
& =f(x, y) \text { otherwise }
\end{aligned}
$$

where $T$ is a prespecified threshold based upon the image SNR.

The current image point is only replaced by the average of its nelghbours if the difference between this average value and the present point brightness is less than a prespecifled threshold. A simple illustration of image smoothing. with the application of a threshold is given in Fig. 5.6 showing the preservation of the edge detail.

In principle convolving masks of any shape and size can be used. Larger masks glve more smoothing (and greater loss of edges) whereas horizontal rectangular masks will smooth horizontal noise but leave noise and edges in the vertical direction relatively unaffected.

\subsubsection{Median Smoothing}

A disadvantage with the threshold method is that it adds to the computation of the smoothing operation. An alternative technique for smoothing in which the edges in on image are maintained is that of median filtering. In this the pixel at the centre of the template is given the median brightness value of all the pixels covered by the template. Fig. 5.7 shows the effect of median filtering on 


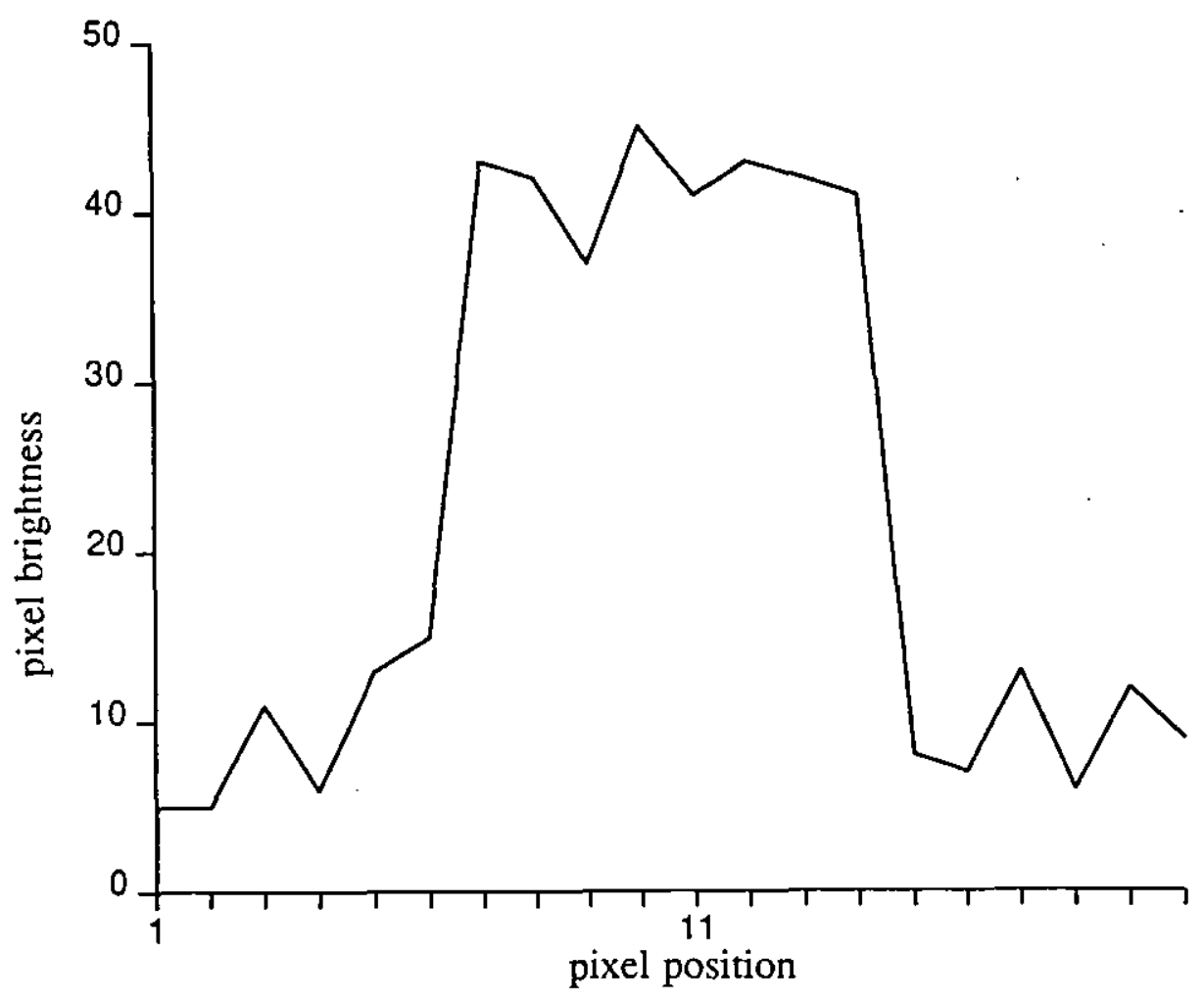

Fig. 5.6 Smoothing using a Threshold.

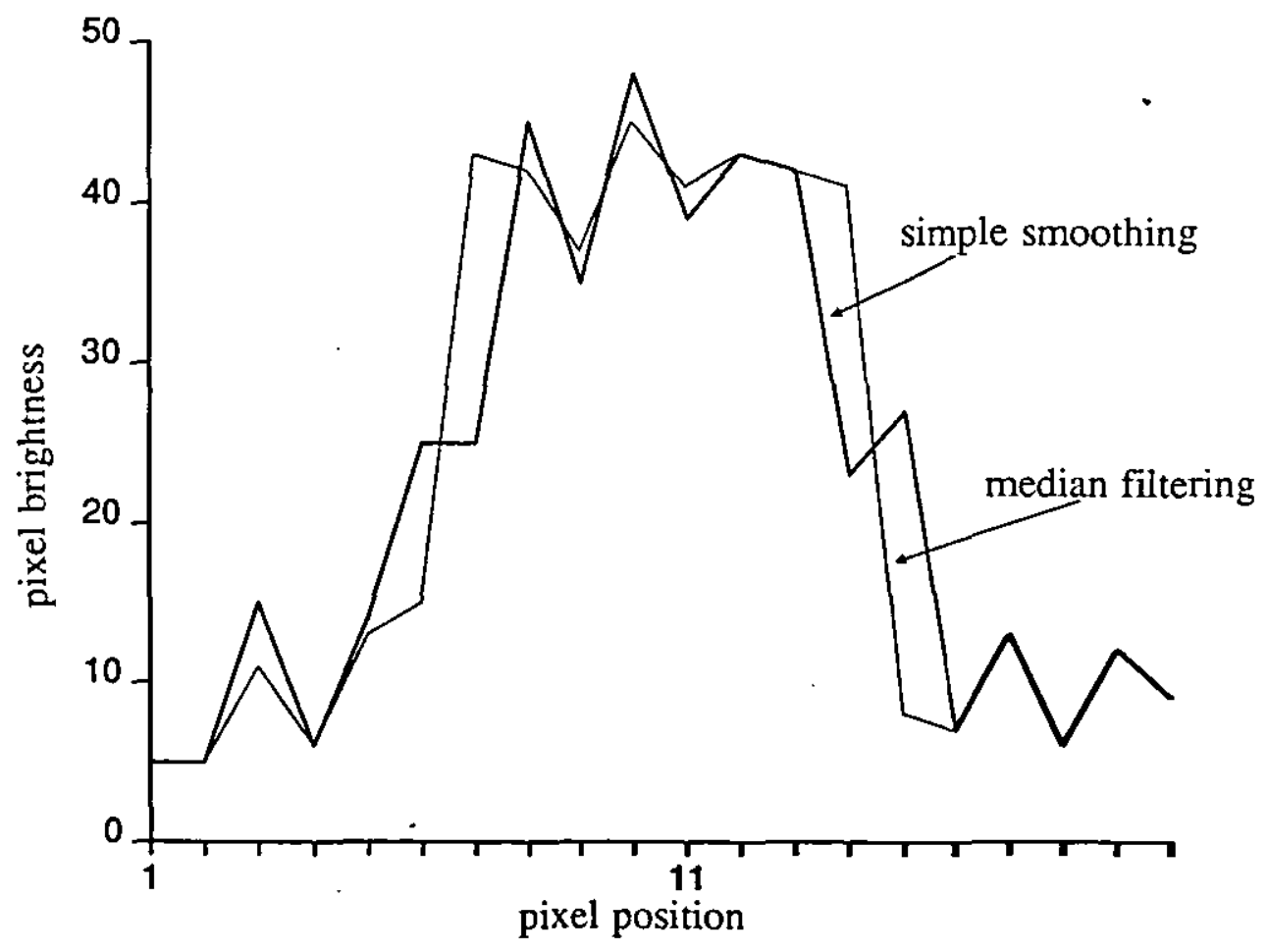

Fig. 5.7 Simple Smoothing compared with Median Filtering 
a single line of image data compared with simple smoothing. Again most of the original edge is preserved.

An application for which median filtering is well suited is the removal of impulse-like noise. This is because pixels corresponding to noise spikes are atypical in the neighbourhood and will be replaced by the most typical pixel brightness in that neighbourhood.

It should be noted that median filtering is not a linear function of the brightness values of the image pixels, consequently it is not a convolution operation in the sense described in the previous section.

\subsubsection{Edge Sharpening}

There are a variety of methods in which the edges in an image may be accentuated by spatial and frequency domain techniques. Sharpening is analogous to differentiation where smoothing is to integration. Images may be sharpened by emphasising the high spatial frequency content of the spectrum, but care must be taken when applying this technique to noisy images since noise is generally of high spatial frequency and accentuated by this operation. One solution is to carry out a smoothing operation before the sharpening takes place. Fig. 5.8 illustrates simple 3 by 3 high emphasis masks.

\subsubsection{Contrast Enhancement}

Contrast refers to a difference in luminance or grey level values in some particular region of an image.

We may alter the grey level values and thus change the 
contrast of the information in an image by effecting a linear or non-linear transformation, i.e. by forming a new image function $g_{2}(x, y)=T\left[g_{1}(x, y)\right]$ for each $(x, y)$, where $T$ is a linear or non-linear mapping.

\subsubsection{Linear Scaling Transformation}

A simple but useful model is the scaling transform. Suppose we have a digital image for which the contrast values do not $f i l l$ the available contrast range. That is. suppose the data covers a range $[\mathrm{m}, \mathrm{M}]$. but that the available range is $[n, N]$. Then the linear transformation

$$
g 2(x, y)=n+\{[g 1(x, y)-m] /(M-m)\}[N-n]
$$

expands the values over the avai lable range.

\subsubsection{Piecewise-Linear Transformation}

A plecewise-linear transformation (PLT) may be a

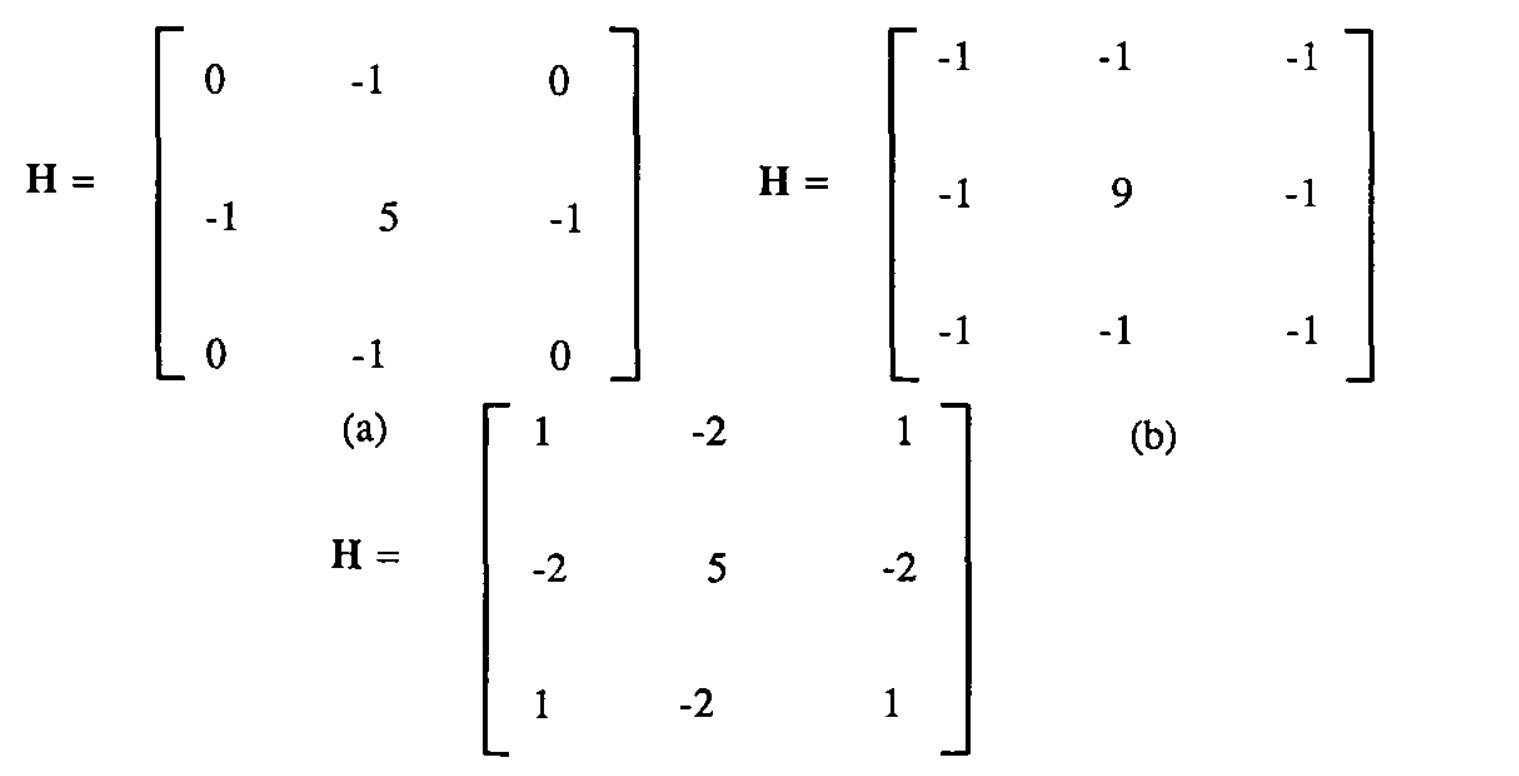

(c)

Fig. 5.8 Some Typical Edge Enhancement Convolution Masks. 
I inear or non-linear mapping. A practical example of the useful ness of this transformation can be illustrated by considering an Image with a low level noise component. The result of applying a three step PLT shown in Fig. 5.9 is illustrated in Fig. 5.10 where the noise component has been reduced and the "target" remains relatively unaffected.

\subsubsection{Histogram Modification}

Useful characteristics of an image may be obtained from the density function, or histogram, of its grey levels. The histogram of a predominantly dark image of low contrast is shown in Fig. 5.11. The contrast of this image may be enhanced by the technique of histogram modification. This term refers to the process of altering the grey level histogram of a given image such that the modified histogram is of a pre-specified form. Histogram equalisation refers to a particular derivative of this technique in which the modified histogram is of uniform magnitude.

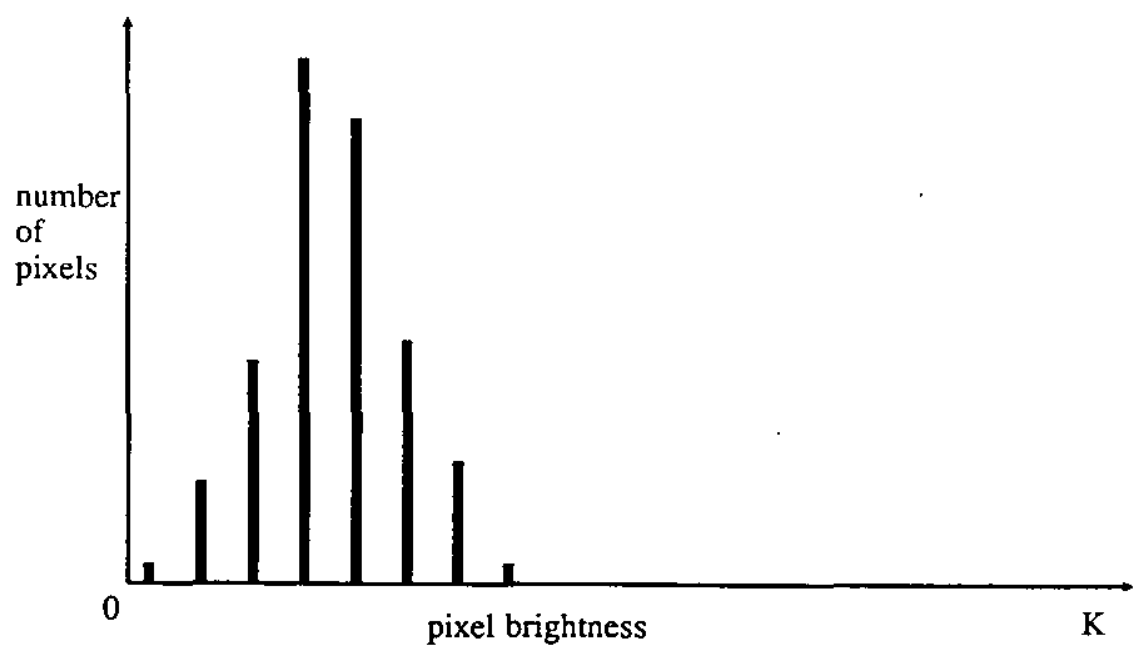

Eig. 5.11. Histogram of Predominantly Dark Image. 


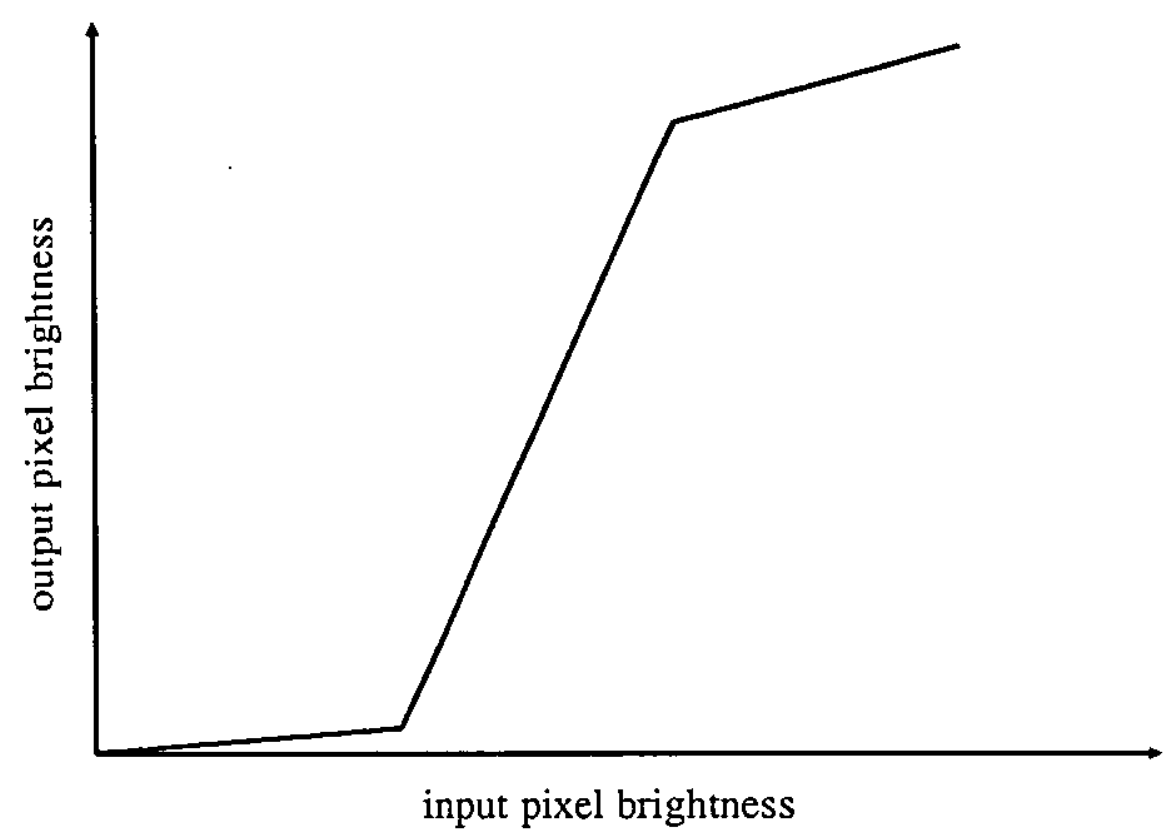

Eig. 5.9 A 3 Step Piece-wise Linear Transformation

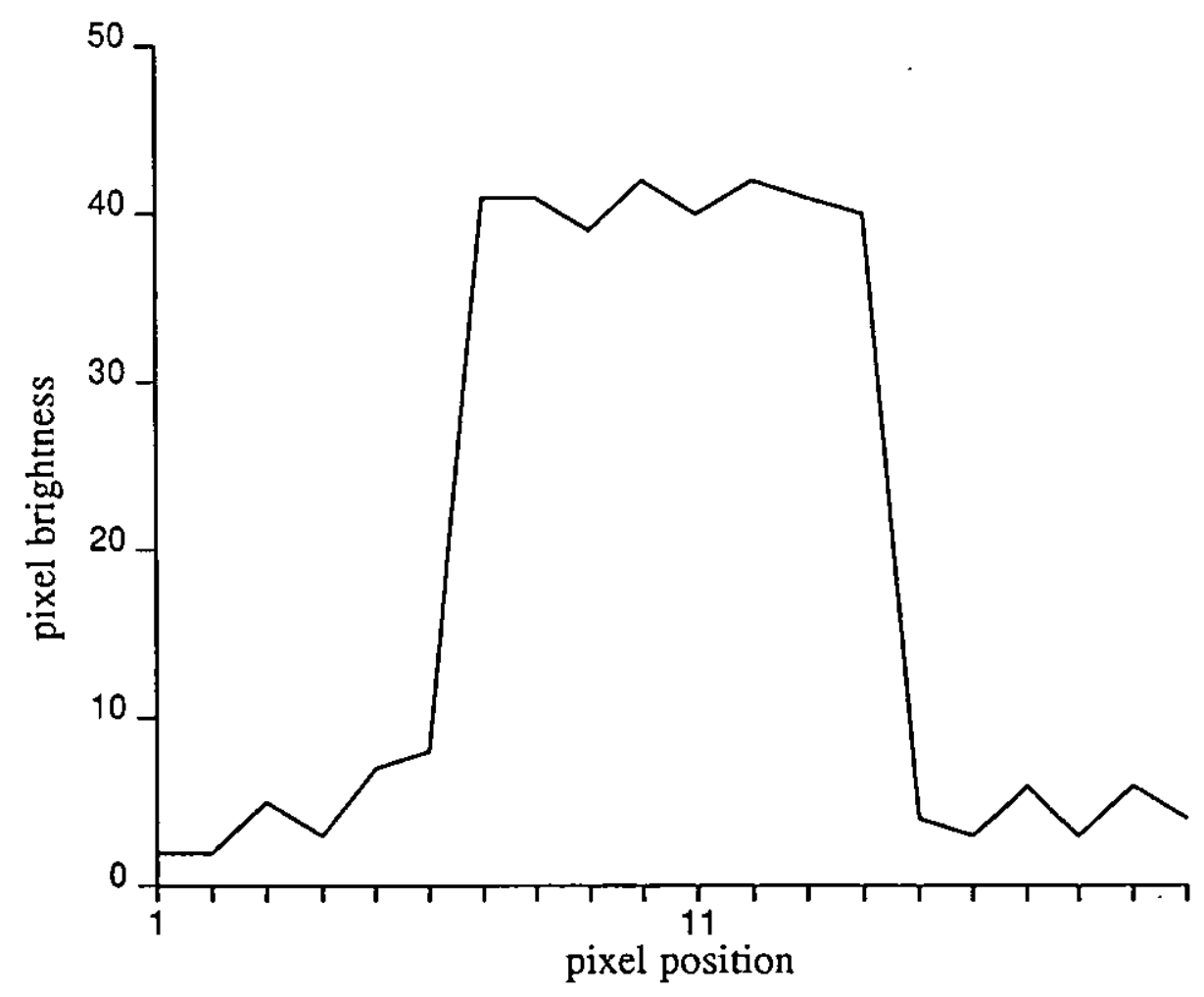

Fig. 5.10 Effects of Applying the Three Step PLT. 


\subsection{Image Restoration}

Image restoration may be described as the reconstruction of a degraded image towards the original by inversion of some degradation phenomena.

One form of a degraded image can be considered as an ideal image, each pixel of which has been spread across neighbouring pixels. The precise form of the blurring at any particular pixel is called the point spread function (PSF). The fourier transform of the PSF is called the Optical Transfer Function (OTP).

\subsubsection{Deconvolution}

Image restoration is simplest when the degradation is point-spread-invarlant (1.e. the PSF is identical for all pixels). If we consider $g(x, y)$ representing a degraded image and $f(x, y)$ is the original, then

$$
G(u, v)=H(u, v) F(u, v)
$$

Now if the transfer function of the degradation is known then in principle the original image may be obtained from

$$
F(u, v)=G(u, v) / H(u, v)
$$

However. H(u,v) may have zeros and this will produce problems if it is used as the divisor. When noise is also introduced, the model becomes

$$
G(u, v)=H(u, v) F(u, v)+N(u, v)
$$

and

$$
F(u, v)=\frac{1}{H(u, v)}\{G(u, v)-N(u, v)\}
$$


In practise it is necessary to consider the restoration filtering to be carried out by some transfer function $M(u, v)$ such that the restored image

$$
F_{r}(u, v)=M(u, v) G(u, v)
$$

A widely used restoring filter is the minimum mean square error (MSE) Wiener filter which seeks to minimise

$$
\frac{1}{N^{2}} \sum_{x=0}^{N-1} \sum_{y=0}^{N-1}\left\{f(x, y)-f^{\prime}(x, y)\right\}^{2}
$$

The Transfer function for this filter is

$$
\begin{aligned}
M(u, v) & =H^{t}(u, v) P_{f}(u, v) \\
H(u, v) & 2 P_{f}(u, v)+P_{n}(u, v)
\end{aligned}
$$

where

$P_{f}(u, v)$ is the power spectrum of the Imoge.

$P_{n}(u, v)$ is the power spectrum of the noise.

$H^{t}(u, v)$ is the transpose of the function $H(u, v)$.

Practical application of this technique however leads to suboptimal performance when the results are judged by the human observer [86]. Power spectrum equalization and geometrical mean filters are alternatives to and improvements upon the Wiener filter [87.88].

\subsubsection{Subtractive Deconvolution}

Images obtained from synthetic aperture systems can often be thought of as a distribution of impulses. The concept of subtractive convolution arises from the idea that the pixels representing the data can be reduced down to the 'noise level' by successively 'subtracting out' each of the spread Impulses [76]. Because noise is never negligible in proctise. it is of course impossible to 
cancel the whole of each spread impulse accurately by a single subtraction. Useful results can often be obtained, however. by proceeding more gradually.

The archetype of subtractive techniques is Hrgbom's simple cleaning algorithm [78].

The actual recorded image $a(x, y)$ is referred to as the 'dirty image' because it is blurred and contaminated with noise. We wish to form a 'cleaned image' corresponding to the recoverable true image. We denote the pixels of the cleaned Image by $f c(x, y)$. Al| the pixels of $f c(x, y)$ are set to zero before the algorithm is initiated.

The $f c(x, y)$ gradually takes on value as information is extracted from the dirty image. The iterations are characterized by a positive constant, $\tau$, called the 'loop gain' and is generally taken to be less than unity. An estimate of the average of the $|a(x, y)|$ or the noise level must be avaliable.

The algorithm consists of the following steps:

51: The brightest pixel. denoted by $a(J, k)$. in the dirty image is found, i.e.

$$
a(j, k)>a(x, y) \quad \text { for all } x \neq j \text { and } y \neq k \quad[5.16]
$$

52: A new cleaned image is formed by the additive operation:

$$
\begin{aligned}
f c(x, y) & +\operatorname{ta}(j, k) \delta(x-j, y-k) \rightarrow f c(x, y) \\
& \text { where } \delta(x, y) \text { is the Kronecker delta. }
\end{aligned}
$$

S3: A new dirty image is formed by the subtractive operation: 


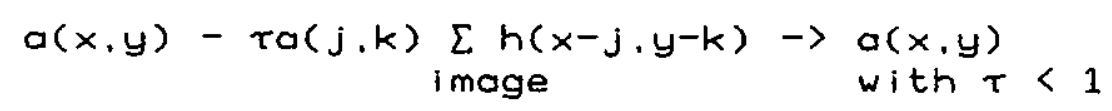

54: return to 51 , unless

$$
a(x, y)<|a(x, y)| \text { for } a \mid 1 x \text { and } y
$$

S5: The cleaned image is convolved with a cleaned PSF. which is $h(x, y)$ with its mainlobe smoothed and its side lobes heavily attenuated to give the best recoverable true i mage.

Fig. 5.12 shows a flowchart of the cleaning olgorithm and Fig. 5.13 and Fig. 5.14 illustrates the effects of this algorithm with a loop goin $r=0.1$ on an image obtained using the SAS system in the tank. The targets appear more sharper than in the or iginal image and the low level noise component in the Image has been reduced. 


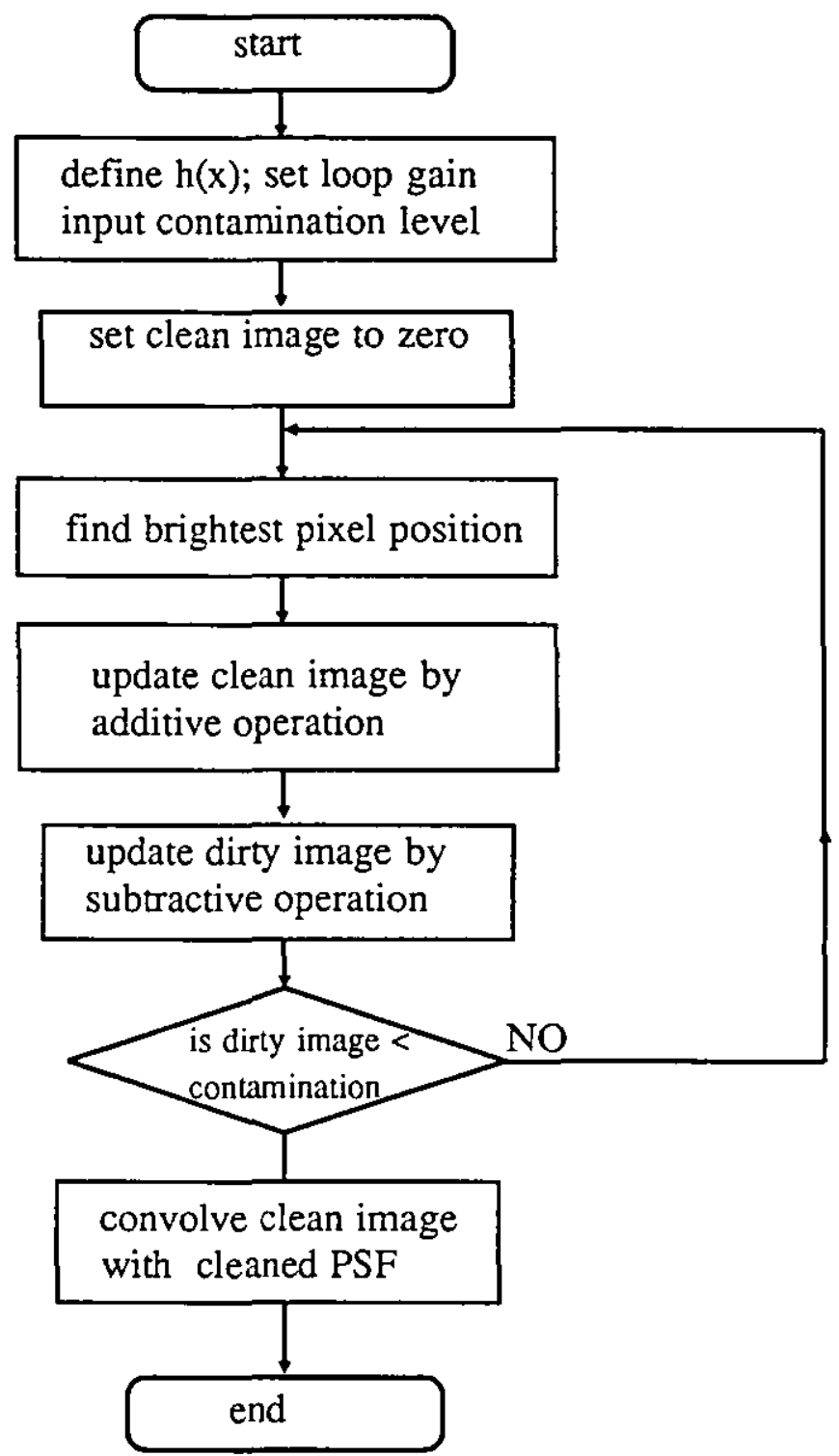

Fig, 5.12 A Flowchart of the Cleaning Algorithm. 

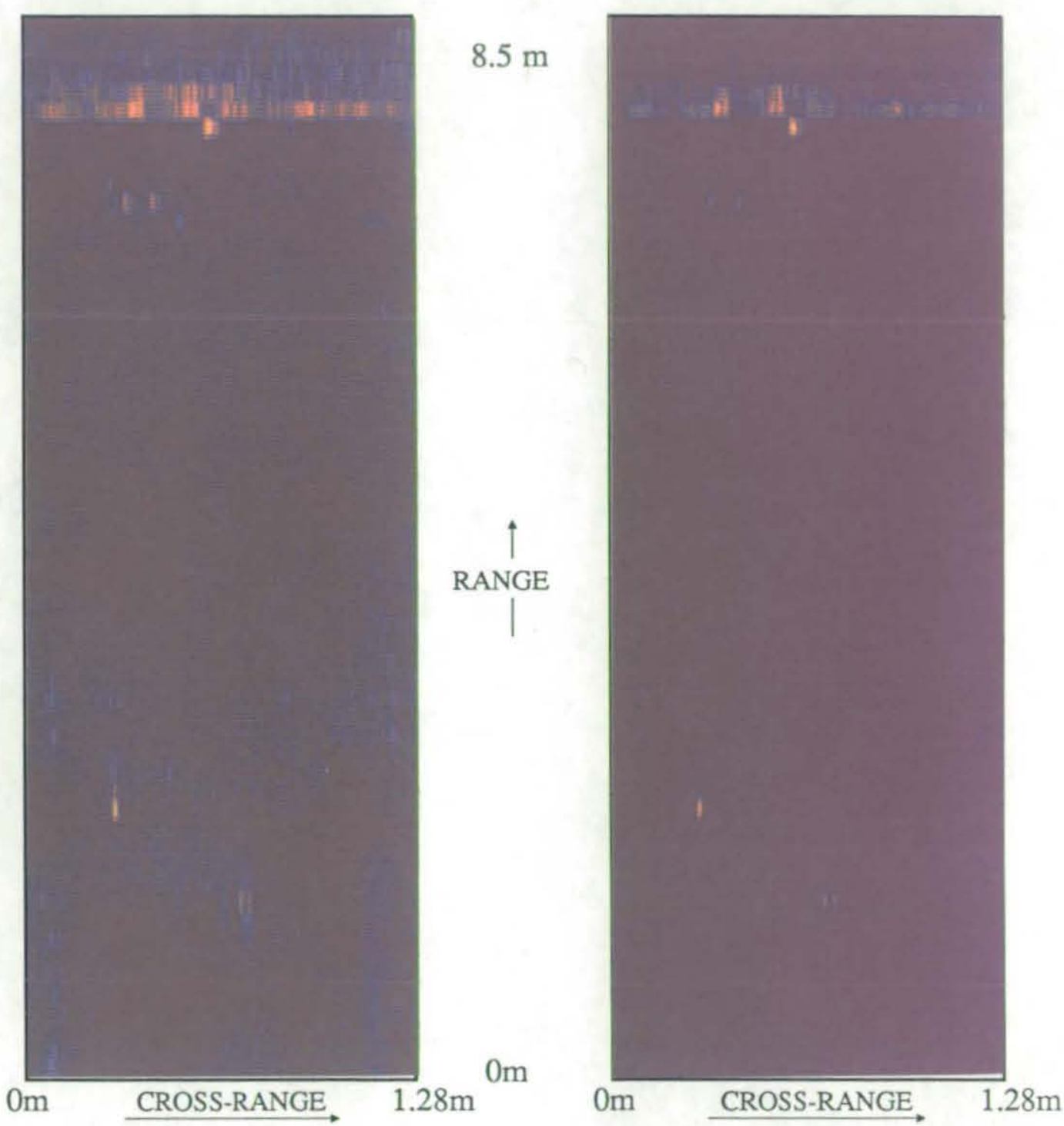

Fig, 5.13 Typical Sonar Image in the Tank.

Fig, 5.14 Sonar Image with the Cleaning Algorithm Applied in the Reconstruction. 


\section{SYSTEM HARDWARE AND SOFTWARE}

\section{I Introduction}

During the period of 1983-87. two versions of the synthetic aperture system have been developed. The first. designed for operation in the department's $9 \mathrm{~m}$ test tank. operates at $150 \mathrm{kHz}$. The second version was derived from the first but operates at $40 \mathrm{kHz}$ and was installed at a local deep water reservoir [73]. This section describes the hardware of the basic system and outlines the data collection and image reconstruction methods employed.

\subsection{Sustem Hardware}

\subsubsection{The 'BBC' Microcomputer with 32016 Co-processor}

A BBC Microcomputer. Which is based round the 6502 microprocessor chip. performs all the control functions of the systems. it controls the stepper motors and triggers the transmit pulse when the platform reaches predetermined positions along the aperture. The BBC microcomputer also performs all the data manipulation functions, moving the data from a temporary buffer to a framestore and saving the data onto disk or transfering images from a framestore to disk. The reconstructed images are also displayed via the BBC microcomputer. Figure 6.1 shows the block diagram of the complete system.

The 32016 co-processor is connected to the BBC 


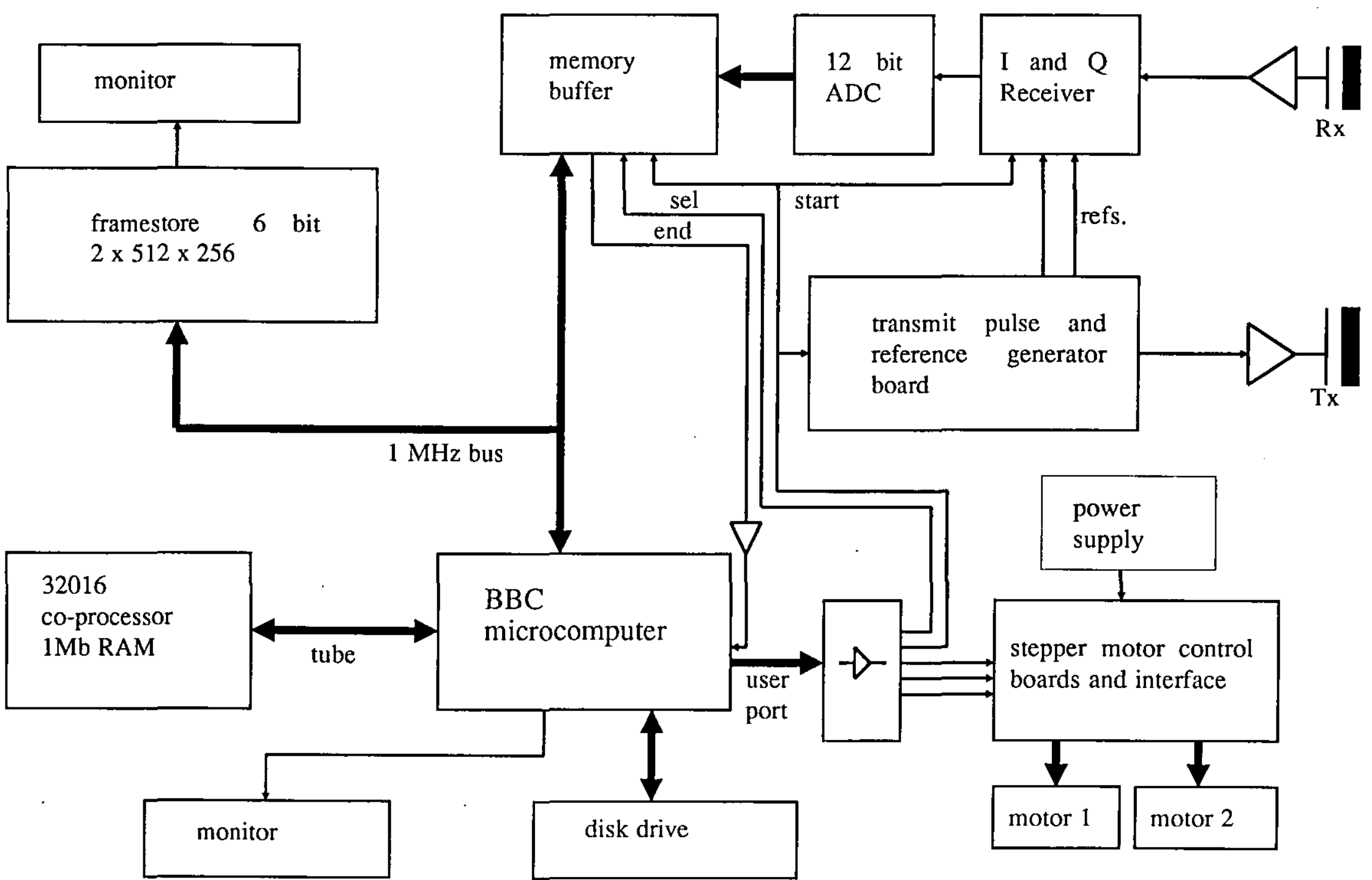

Fig. 6.1 Synthetic Aperture Sonar System Block Diagram 
microcomputer via the TUBE interface and the framestore and memory buffer is accessed using the $1 \mathrm{MHz}$ BUS [60-65]. The control signals for the stepper motors and the trigger for the transmit pulse are sent using the USER PORT (an eight bit programmable bi-directional user interface).

The image is reconstructed using the 32016 co-processor facility. This 32 bit processor has 1 Megabyte of RAM, operates at $6 \mathrm{MHz}$ [66]. and in addition contains a hardware floating point multiplier making it well suited for signal processing applications. Programs to reconstruct the images were written in Fortran IV with some calls to high speed data transfer rout ines in assembly language. These fast subroutines proved necessary to optimise the communications between the BBC microcomputer. the 32016 co-processor and a British Telecom designed framestore.

\subsubsection{The $I$ and $Q$ Baseband Receiver Board}

Quadrature sampling is used in the receiver to reduce the sampling rate and data storage requirements. In this technique the incoming signal is translated into two baseband channels and separately sampled, as discussed in section 3.3.3.

The received signal is fed through an amplifier stage with a gain $24 \mathrm{~dB}$ and divided into two identical channels. These two channels are used to extract the quadrature baseband components of the received signol. A block diagram of the $I$ and 0 recelver board is shown in figure 6.2 .

In each channel the signal is fed to its own analogue multiplier (RS1495). Reference signals with an amplitude 


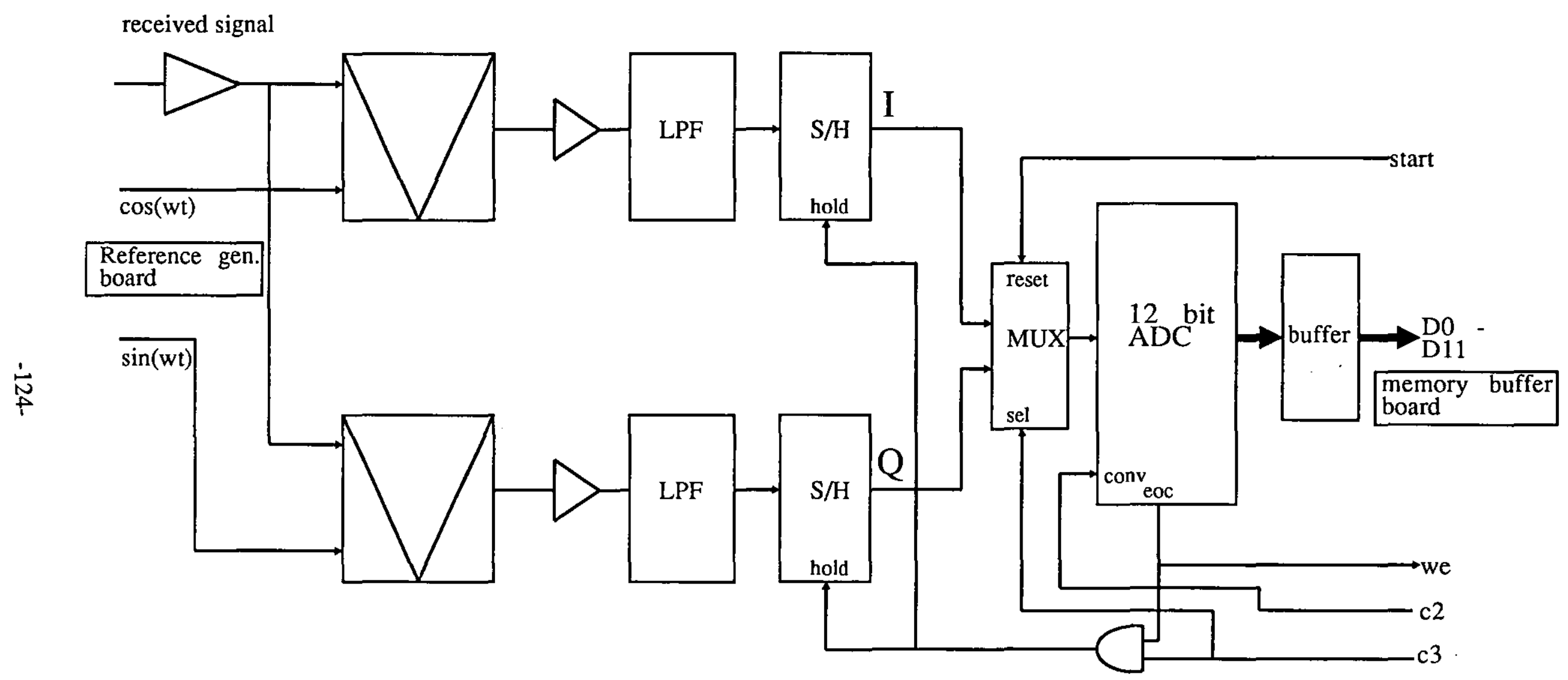

Fig. 6.2 Block Diagram of the Baseband I and Q Receiver Board 
of 20v $p-p$, generated on the transmit pulse and reference generator board are the second input to each analogue multiplier. The reference signal is a cosine wave of the same frequency and phase as the transmitted signal for the $I$ channel and a sine wave for the $Q$ channel. Each multiplier has offset adjustments for both the inputs and the output. Any zero offset errors on the $I$ or $Q$ channels are removed using the offset adjustment controls. The multiplier outputs are buffered by ultra low nolse. high speed operational amplifiers (OP-37).

The low pass filter used to remove the sum components of the multiplication, is a 4 th order Butterworth active filter giving $-24 \mathrm{~dB} /$ decade attenuation. The cut of frequency is $5 \mathrm{kHz}$ but this can easily be altered by changing the values of the resistors used [80].

Two sample and hold amplifiers (LF398) are latched simultaneously and hold the $I$ and $O$ signal values at the appropriate instances in time. An analogue switch is used to multiplex the two channels to a 12 bit ADC (AD574) having a conversion time of less than $25 \mathrm{\mu s}$. This device is configured to convert signals in the range $-5 v$ to $+5 v$. The digital output of the ADC is sent to the memory board via a ribbon cable. The end of conversion ( $E O C$ ) signal from the $A D C$ is used to initiate the write enable (WE) pulse for the memory buffer.

A programmable crystal oscillator. on the memory board, is the source of the timing signals for the $A D C$. $S / H^{\prime} s$, and the multiplexer.

Figure 6.3 shows the various synchronising waveforms found on the $I$ and 0 receiver board and the memory buffer board. The select (SEL) signal is set by the BBC 


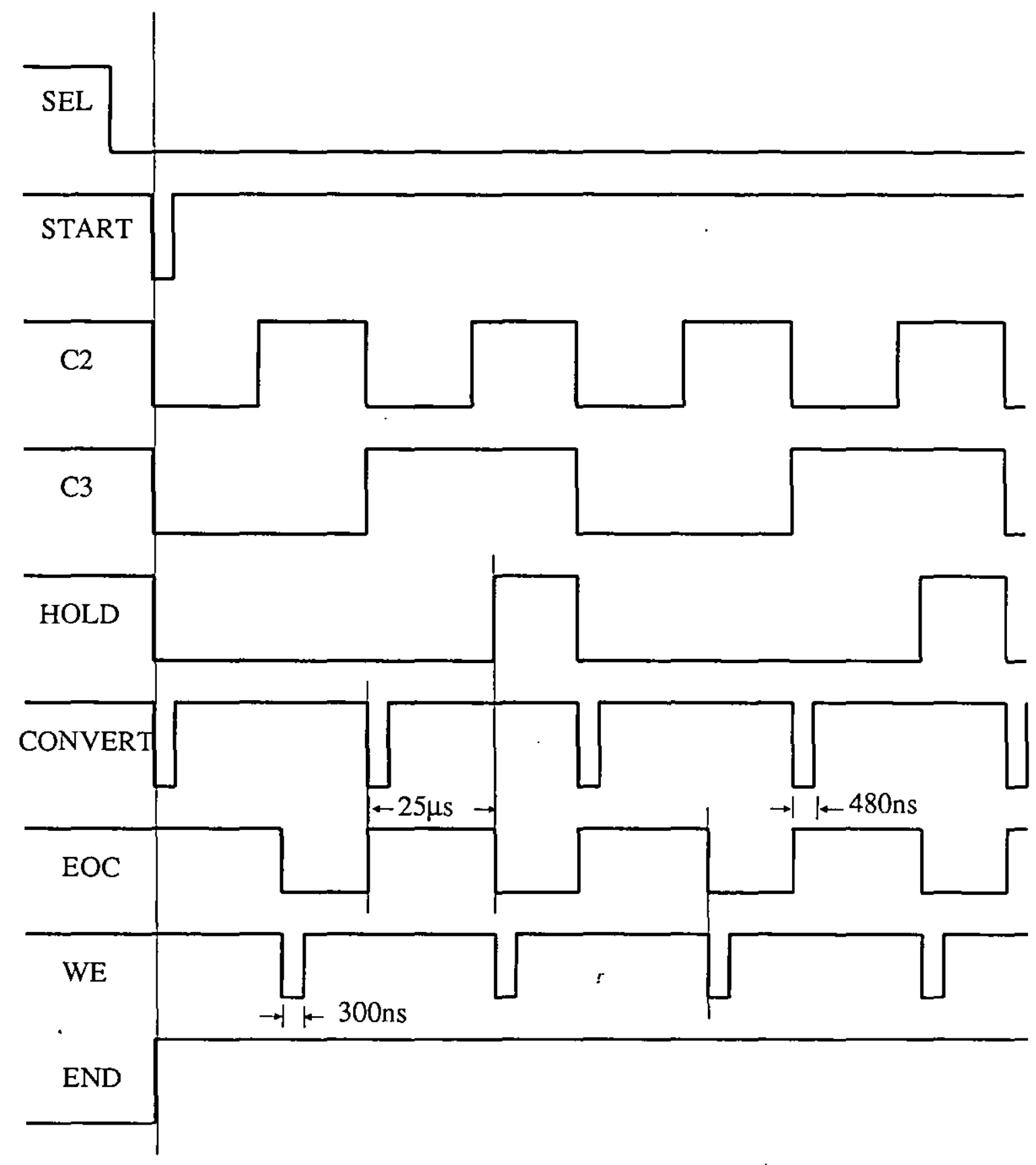

Fig, 6.3 System Timing Waveforms 
microcomputer via bit 3 of the user port (PB3) and determines whether the $8 B C$ microcomputer has access to the memory buffer or not. If SEL is high the BBC microcomputer has access to the data bus and address bus of the memory buffer. If SEL is low then upon reception of the write enable (WE) signal. the output of the ADC is loaded into the memory buffer. at the location specified by the address generator.

The negative edge of the START signal (PB4) synchronises all the various activities of the system; the programmable oscillator is reset and enabled, and the address generator is also enabled. The $c 2$ and $c 3$ signals are derived from the programmable oscillator by the use of a frequency divider ( $74 L 5161 A N$ ). The negative edge of $c 2$ initiates the CONVERT signal to the $A D C$ and $c 3$ is used to trigger the HOLD signal for the sample and hold amplifiers.

The end of conversion (EOC) signal generated by the $A D C$ triggers the WE signal for the memory buffer. When the address generator reaches the last required memory location (or end of the memory buffer) an END signal is generated. The BBC microcomputer detects the END signal using $P B O$ and takes control of the memory buffer. by setting SEL to high and transfers the data held in the memory buffer into the framestore.

The input-output characteristics of the receiver board are shown in Fig. 6.4 (relative to $1 \mathrm{~V}$ ) and show that the abtainable dynamic range is $67.5 \mathrm{dBs}$ compared with the expected value from a 12 bit device of 72 dBs. This difference in the value of the dynamic range is due to conductive and radiated noise from the $B B C$ microcomputer and the other digital parts of the system. Separate ground planes and decoupling capacitors were used to reduce the 
nolse.

The full circuit diagram of the receiver board is shown in appendix 8 .

\subsubsection{The Memory Buffer Board}

The memory buffer board contains 1 Kbyte of 12 bit static RAM. facilitating the storage of 512 time samples of the received signal from each transmission. (Two 12 bit numbers are stored, the $I$ and 0 quadrature components for

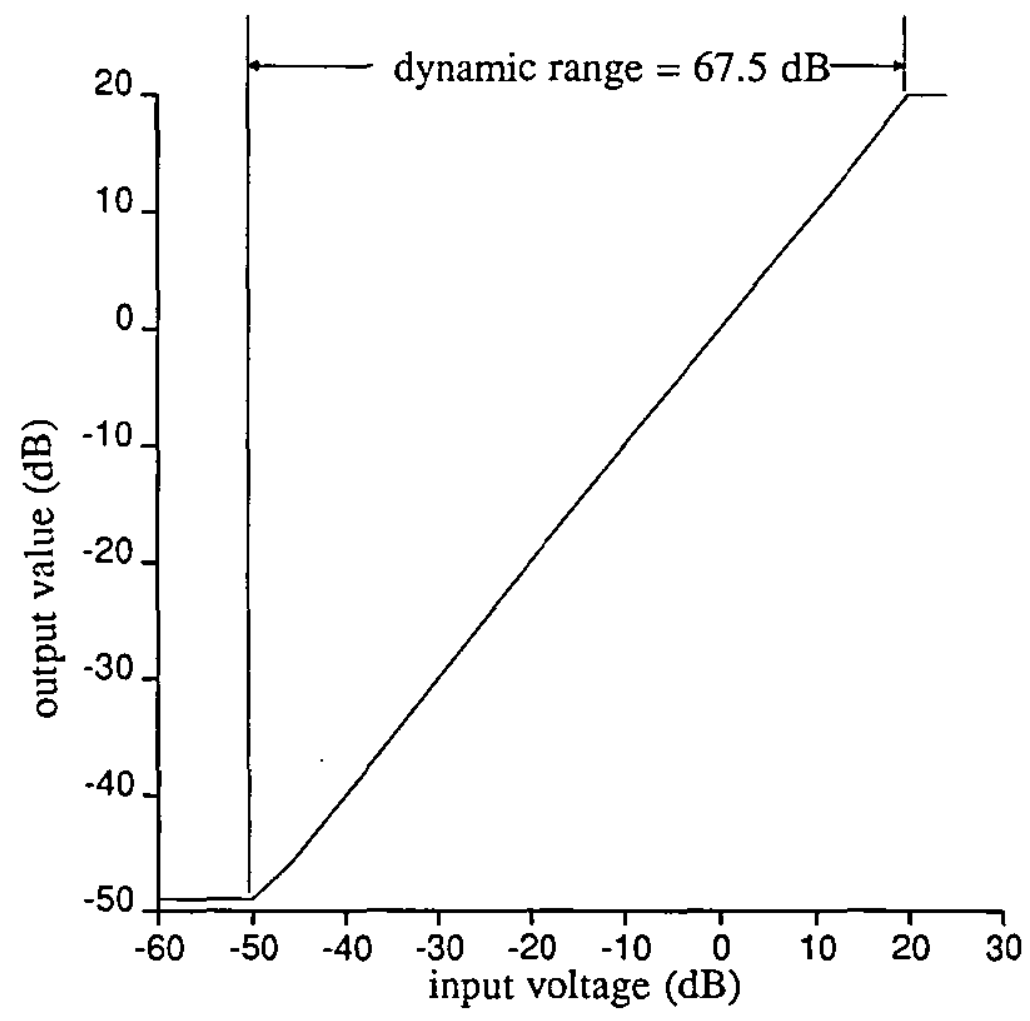

Fig, 6.4 Input-Output Characteristics of the Receiver Board.

each time sample of the received echo).

A block diagram of the memory board is shown in Fig. 6.5.

A precision crystal oscillator with programmable 


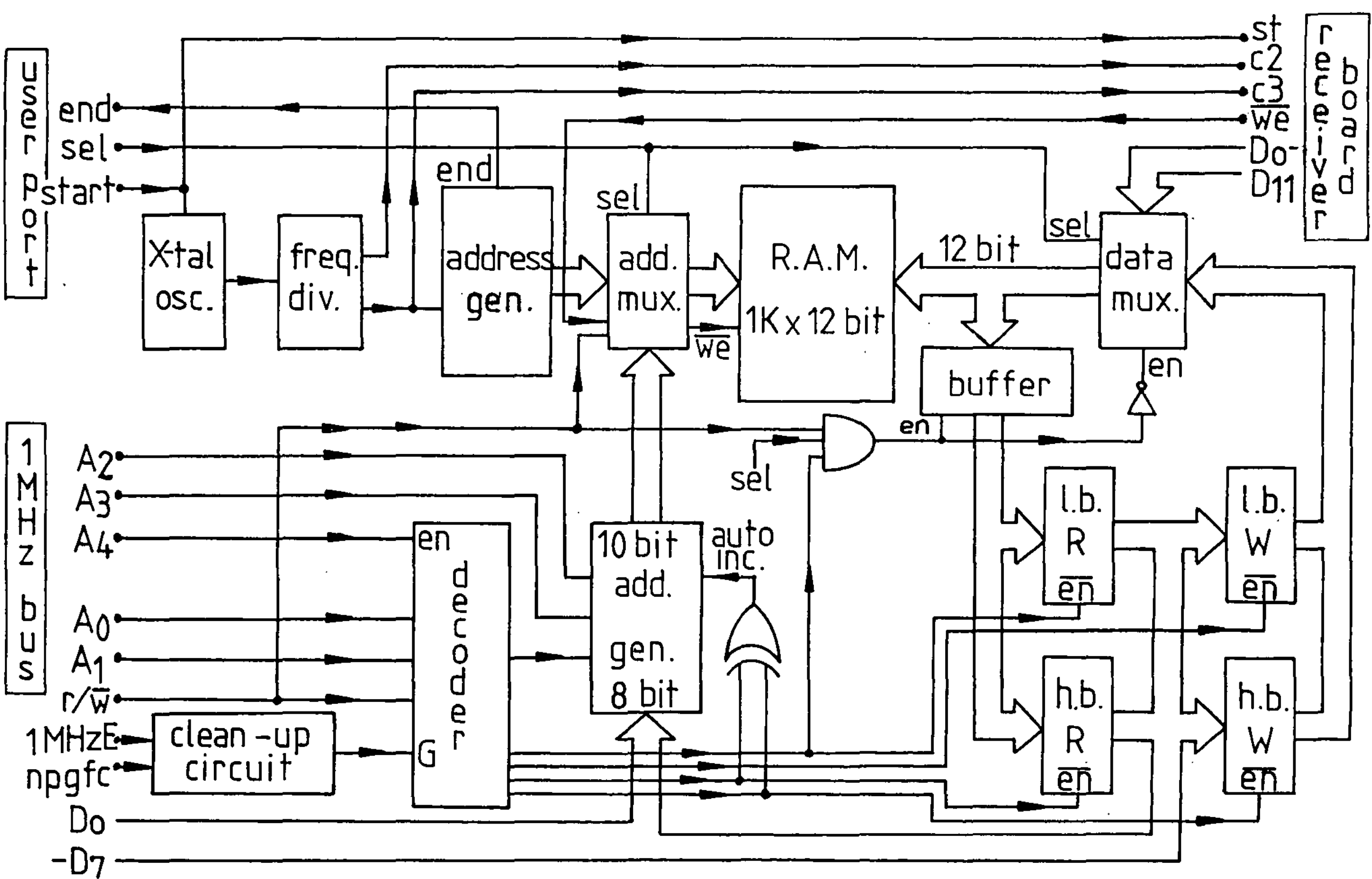

Fig. 6.5 Block Diagram of the Memory Board. 
prescaler (PXO-1000) is the source of all the timing signals and synchronises the various activities of the receiver hardware.

$A$ select ( $S E L$ ) signal from the BBC microcomputer user port controls whether the BBC microcomputer has control of the memory buffer or not. If the BBC microcomputer is not in control. the programmable osclilator is enabled. and upon reception of the WE signal from the ADC, the data generated by the $A D C$ is stored in the memory buffer at the location specified by the address generator. The I component of the baseband signal is stored first and the 0 component is stored in the next consecutive memory location.

When the address generator reaches the last available memory location, an end (END) signal is sent to the $88 C$ microcomputer via the user port. This indicates that the memory buffer is full and can be accessed by the BBC microcomputer for data removal.

If the BBC microcomputer is in control, the buffer address and data are provided and transferred using the $1 \mathrm{MHz}$ bus. The 12 bit data is divided into two 6 bit numbers and stored in two consecutive locations in the framestore. The two MSB's of the 10 bit buffer address are defined by the $A 2$ and $A 3$ address lines of the $1 \mathrm{MHz}$ bus, the 8 LSB's of this address are obtained from the 8 bit data bus.

A hardware based auto-increment facility is used to reduce the time taken to save or load data to or from the memory buffer. The memory buffer address is specified on the first read or write operation, and on each consecutive access, the memory buffer address is 
automatically incremented ofter each high byte read or high byte write.

A full circuit diagram of the memory buffer board is shown in appendix 7 .

\subsection{Range Select Facility}

The range select facility is used to select which particular set of time samples, of the returning echo. to be stored. Normally the first 511 time samples are stored in the memory buffer.

Consider the situation where a target. ot a very large range. is of interest, then:

(i) a very large memory store is required to save the large quantity of data generated by the large number of time samples.

(ii) If a reduction in range resolution is acceptable. then the echo can be sampled at a slower rate and a smaller memory size is required.

(iii) a relatively small memory is used and the nearest time samples are disregar ded and not soved.

A decrease in range resolution was not acceptable, and to keep the range resolution as close to cross range resolution as possible. option (iii) was used.

SW7 and SW8 ( on the memory board) select the set of time samples stored in the memory buffer. Table 6.1 tabulates the switch settings required for saving the particular time samples of each transmission. The time samples can be converted to values in range with the 
knowledge of the sampling frequency.

$\begin{array}{cc}\frac{S W 7}{1} & \frac{5 W 8}{0} \\ 0 & 1 \\ 1 & 1\end{array}$

TIME SAMPLES STORED

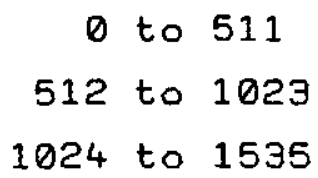

Table 6.1 Switch Setting for Range Select Options

\subsubsection{The Transmit Pulse and Reference Generator Board}

This board contains the elements to generate the transmit pulse and the reference signals and a block diagram of the board is shown in Fig. 6.6. The quantized value of a cycle of each of the waveforms are stored in three ( 32 by 8 bit) PROMs. A voltage controlled oscillator (VCO) feeds a chain of counters which generate the PROM addresses. A $10 \mathrm{~V}$ precision reference is provided for the digital to analogue convertors (DACD800). These produce analogue outputs of 20 volts $p-p$ which when buffered and low pass filtered by the high speed op-amps (LM318) supply the transmit pulse and the sine and cosine reference signals to the system. Fig. 6.7 shows the amplifier circuit for the transmit pulse used in the department tank.

A trigger is sent by the BBC microcomputer. Via the user port. when the platform reaches one of the positions in the synthetic aperture, to initiate the vCo. The VCo controls the frequency of the signals and can be varied by the control of a potentiometer. This is very useful when the system is to be used with different transducers - the frequency can be easily altered to match the transducer's. The integral number of cycles (up to 255) in the transmit pulse is preset on a set of DIL switches. The reference 


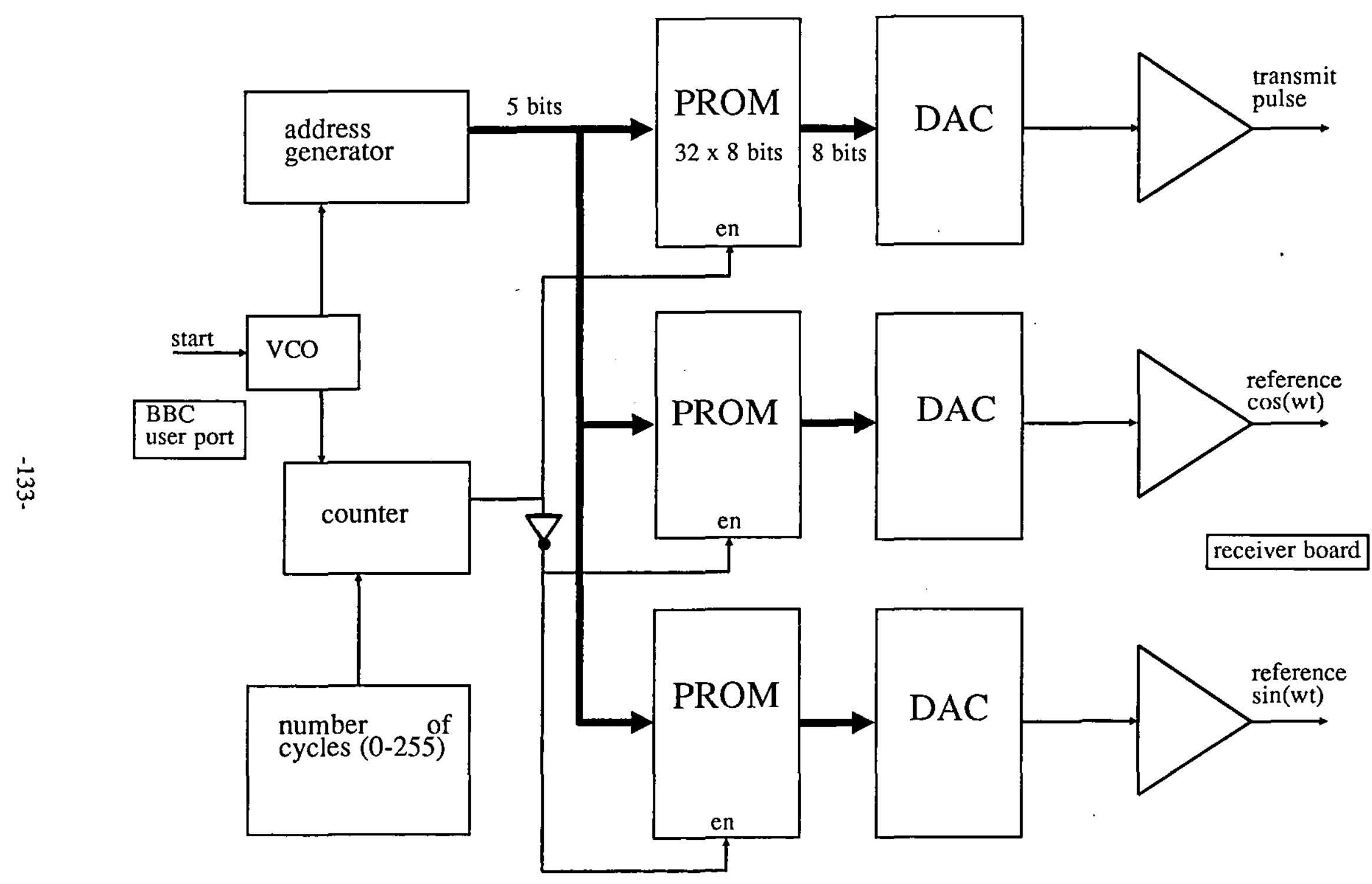

Fig. 6.6 Block Diagram of the Transmit Pulse and Reference Generatore Board. 


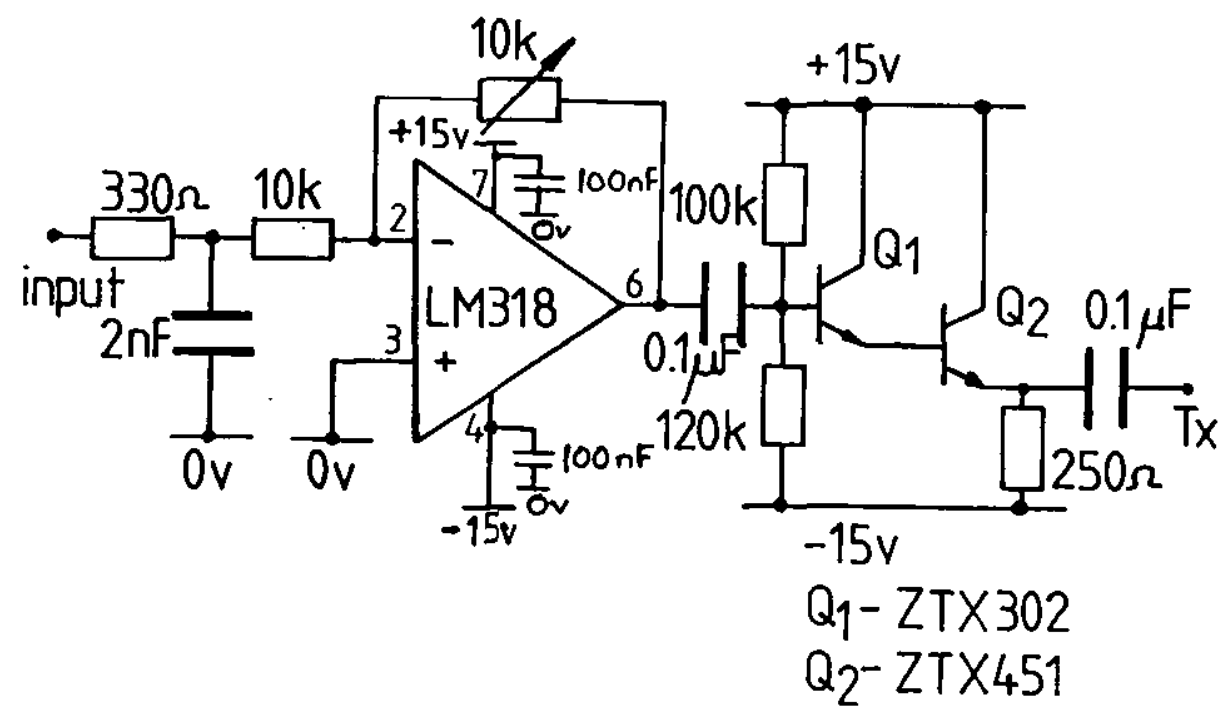

Eig.6.7 Transmit Pulse Amplifier Circuit used in the Department Tank.

signals are disabled for the duration of the transmit pulse, to provide a zero reference (at the output of the analogue multipliers, for the system. This is used to reduce errors due to zero drift, by the measurement of the value of the zero, on each transmission and then using this value in the reconstruction process.

A full circuit diagram of the transmit pulse and reference gemerator board is shown in appendix 6 .

\subsubsection{The Stepper Motors}

An aperture of up to $4.5 \mathrm{~m}$ can be synthesized in the tank Laboratory, as illustrated in Fig. 6.8. Two stepper motors drive 16 D.P., 15 T.P.I. steel gears and 


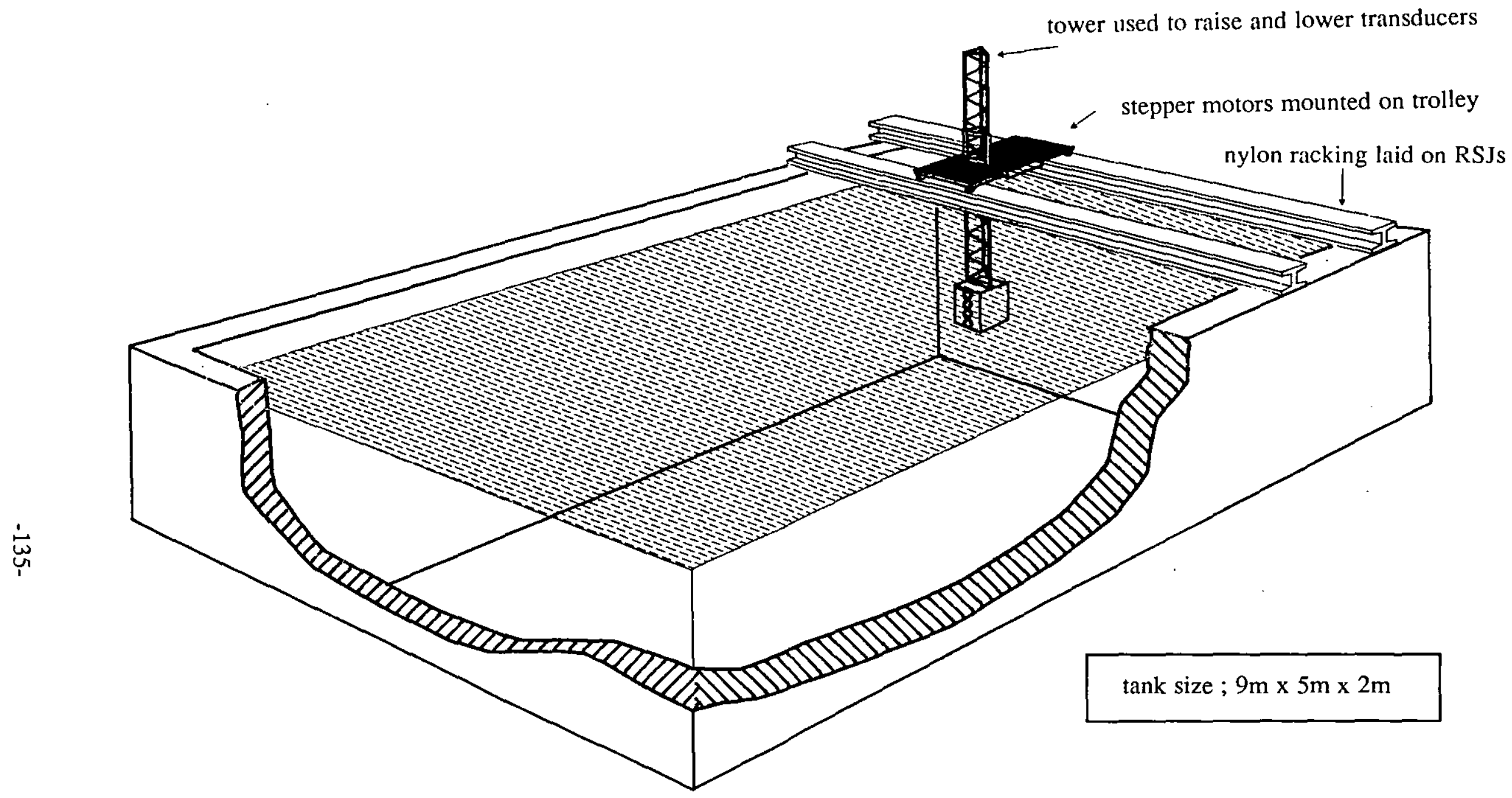

Fig. 6.8 Cut out View of Department tank. 
the gears engage $1 / 2$ " by $1 / 2$ " nylon racking laid on girders at one end of the tank. A weight is used to reduce vibration as the platform traverses across the tank. The transducers are fixed at the bottom of a tower which is mounted on the platform. The tower can be raised or lowered in the water to the desired depth. The speed and direction of the platform is set by the BBC microcomputer controlling the stepper motors via the user port. Pb7 (bit 7 of user port) is used to set the speed of movement. as an internal timer in the BBC can be configured to output square wave pulse on $\mathrm{Pb} 7$. The output of $\mathrm{Pb} 7$ is under interrupt control and leaves the BBC microcomputer free to carry on with the other control tasks and data manipulation. Pb6 is used to determine the direction of movement and Pb5 the mode of the stepper motors. The mode control enables a 200 steps/rev. stepper motor to be used in a 400 steps/rev. mode.

Detalled specification of the stepper motors is included in Appendix 3.

\section{3 Sustem Software and Operation}

This section discusses the software and procedures for collecting the data in the tank laboratory and then the process of reconstructing the final image.

\subsection{The Data Collection Procedure}

The overall control of the system is performed by the BBC microcomputer using programs written in Basic. Subroutines in assembly language are used to handle the tasks that require fast and accurate timings, for example. stepper motor control and data transfer between the memory 
buffer, the BBC microcomputer and the framestore.

When an aperture is to be synthesized, the frequency of the transmit signal and the sampling frequency (or the range cell) are set using the $V C O$ and the precision programmable crystal oscillator. respectively. The number of complete cycles in the transmit pulse are also selected. The following parameter are then chosen:- aperture length, direction and speed of transverse. and the inter-element distance. (normally $\lambda / 2$ ). Once initiated the platform moves along the aperture, at the preset velocity. At each element position, the BBC microcomputer sends a pulse to trigger the transmit pulse. The address generator for the memory buffer is also enabled $(S E L=10 w)$. The recelved waveform is converted into its baseband quadrature components and the two signais ( I and 0) are converted into 12 bit digital values and stored in the memory buffer. When the memory buffer is full and all the required time samples of that particular transmission have been stored, the $B B C$ microcomputer transfers the data held in the memory buffer to the framestore.

On each transmission, the duration of the transmit pulse is used to adjust for any drift in the zero values of the $I$ and $Q$ signals. The cosine and sine reference signals are disabled during the transmit pulse and when this is fed through the analogue multipliers and low pass filtered. it defines the digital value of the zero of the I channel and the 0 channel. These zero values are stored and used during the reconstruction process.

The value of the I component is stored in the first location and the 0 value is stored in the adjacent location. The values of $I$ and 0 are stored as two 6 bit bytes (the framestore has a 6 bit display and storage 
capacity). the low byte first and the high byte mext. Fig. 6.9 shows the format of the data stored in the framestore. When the platform reaches the end of the aperture, the stepper motors are disabled and the data stored in the framstore can be saved and/or processed using the second processor. A flowchart of the data collection process is shown in Fig. 6.10.

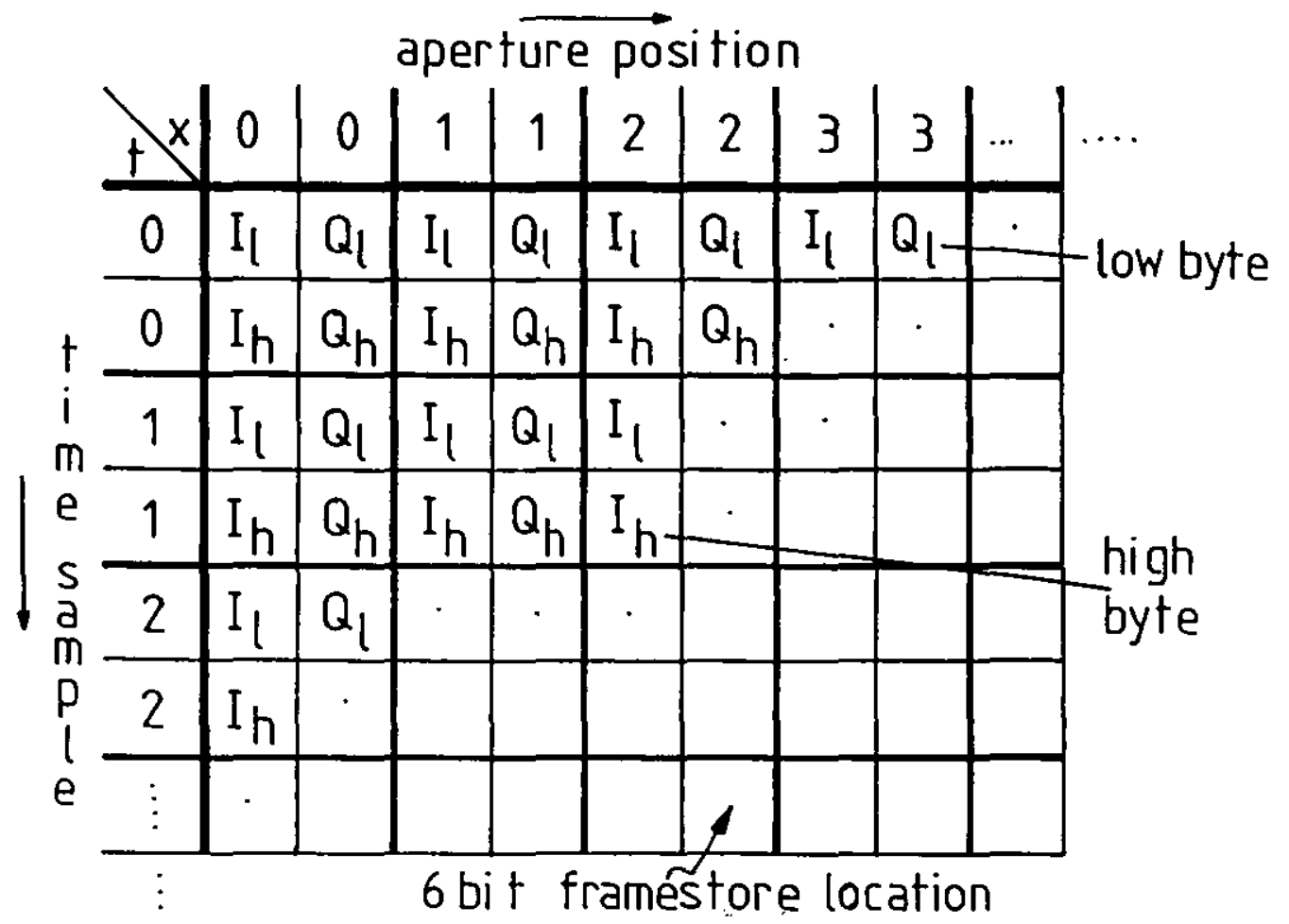

Fig. 6.9 Data Storage Eormat in the Eramestore.

\subsubsection{Image Reconstruction using the Co-processor}

The reconstruction of a target involves the summation of the delayed signals, arriving at each aperture element position. The delay is proportional to the distance between the image point and the element position.

Consider the medium being isonified by a transmitted signal $S(t)$, originating from element position $P,\left(x_{0}, y_{0}\right)$. in the synthetic aperture shown in Fig. 6.11. The 


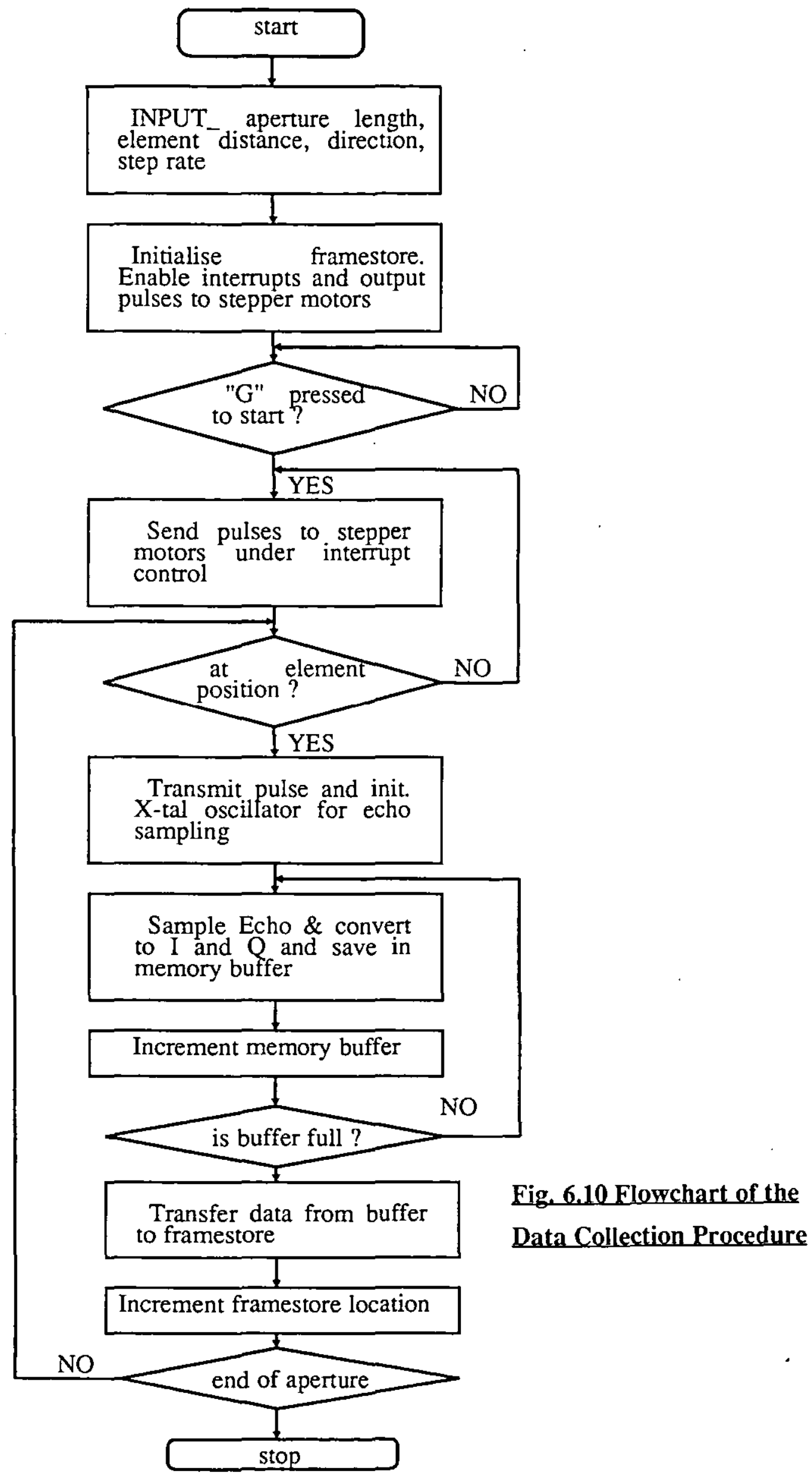


received signal due to a point target. $T_{1}$, at $\left(x_{1}, y_{1}\right)$ is

$$
R\left(x_{\sigma}, y_{\sigma}, t\right)=S\left(t-\frac{2 z 1}{c}\right) \exp \left(j 2 \pi f_{0}\left(t-\frac{2 z 1}{c}\right)\right) \quad[6.1]
$$

where $z 1$ is the distance between the target. $T_{1}$, and the element position. $P$. in the aperture and ignoring all attenuation and scattering effects of the medium.

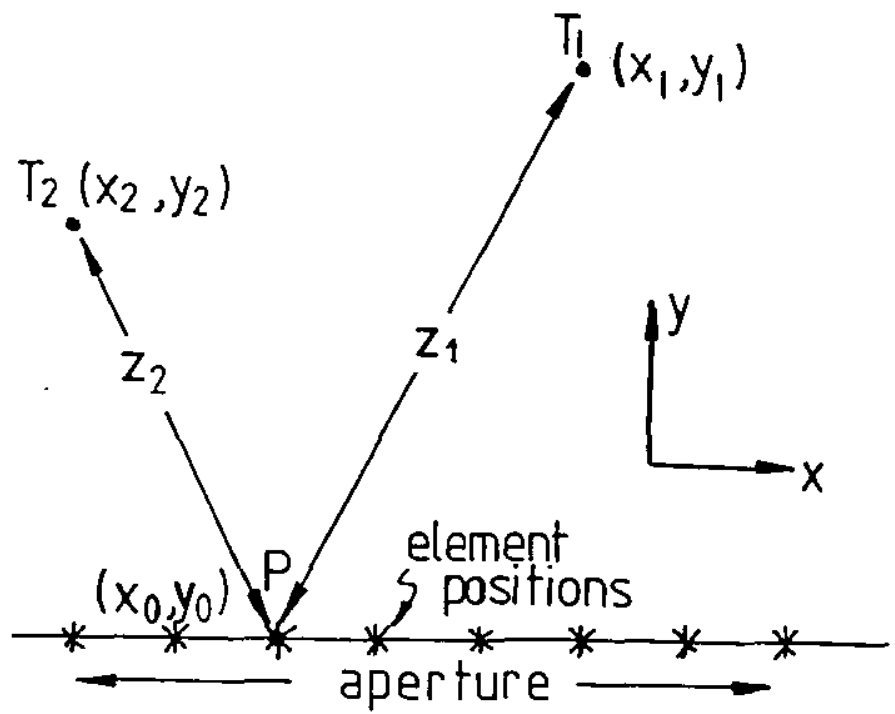

Fig.6.11 Image Reconstruction Geometry.

The imaging process to reconstruct an image point $P_{2}\left(x_{2} \cdot y_{2}\right)$ is performed by summing the received signals over the synthetic aperture length at the proper points in time. The proper points in time are the precise instants when the pulse centre would arrive at that element position after reflecting off a point target at $\left(x_{2}, y_{2}\right)$.

The time instant 


$$
t 2=\frac{2 z 2}{c}
$$

where $z 2$ is the distance between the point $T_{2}$ and the aperture position $P$.

Another aspect of the reconstruction process is that the signals received at the aperture positions are shifted in phase due to the differing distances between the target and each aperture position and cannot simply be summed. One method of phase correction is to perform a complex multiplication on the complex baseband received signal prior to summation by

$$
\exp \left[j \frac{\left.\left(4 \pi f_{j} z 2\right)\right]}{c}\right.
$$

The reconstruction of an image point can then be described as

$$
I\left(x_{2}, y_{2}\right)=\sum R^{\prime}\left(x_{0} \cdot y_{0} \cdot t=\frac{2 z Z}{c}\right) \exp \left(\frac{j \pi f_{0} z Z}{c}\right)
$$

where $R^{\prime}$ is the complex baseband received signal. The complex baseband signal is used because it offers the potentiality of a much reduced sampling rate and data storage as discussed in section 3. 3. 3 .

In practise the complex multiplication involves four real multiplications and two additions. The "proper instant" in time criterion involves the processing of data lying in a hyperbolic arc in the data array. The shape of the hyperbolic arc is dependent upon the shortest distance between the image point and the aperture. Considerable time is taken in the calculation of the shape of the arc and in using the data lying on that arc to reconstruct the particular image point. Hence the processing time for image reconstruction is reduced by the calculation of the hyperbolic arc for a particular value of the distance 
between the image point and the aperture and reconstructing of the image points lying in the same range cell and translating the shape of the arc along the data array. as different points in the cross range axis are reconstructed.

Image reconstruction using the co-processor is achieved using programs written in FORTRAN. A flow chart of the procedure is shown in Fig. 6.12.

\section{4 The Development of a $40 \mathrm{KHz}$ System}

Once the $150 \mathrm{kHz}$ system was working satisfactorily in the tank laboratory and to prepare for the trials at a local reservoir (Foremark Reservoir). the system was redesigned to operate at $40 \mathrm{kHz}$. This lower frequency was chosen because a larger wavelength meant a greater resilience to motion errors. A lower absorption coefficient was also advantageous due to the larger ranges invalved at the reservoir.

To adapt the system to operate at $40 \mathrm{kHz}$ required the changing of only a few components as the design of the system involved the concept that it would operate over a large frequency band. This included minor alterations to components used in the various filters to optimise the system operation at this lower frequency.

The configuration shown in Fig. 6.13 was used to generate a vertical aperture up to $20 \mathrm{~m}$ in length using two parallel $6 \mathrm{~mm}$ diameter polypropelene ropes as guides for the frame which holds the transducers. The ropes are kept tout and vertical by using concrete weights and the frame is moved up and down by using two stepper motors controlled by the BBC microcomputer, to drive a large 


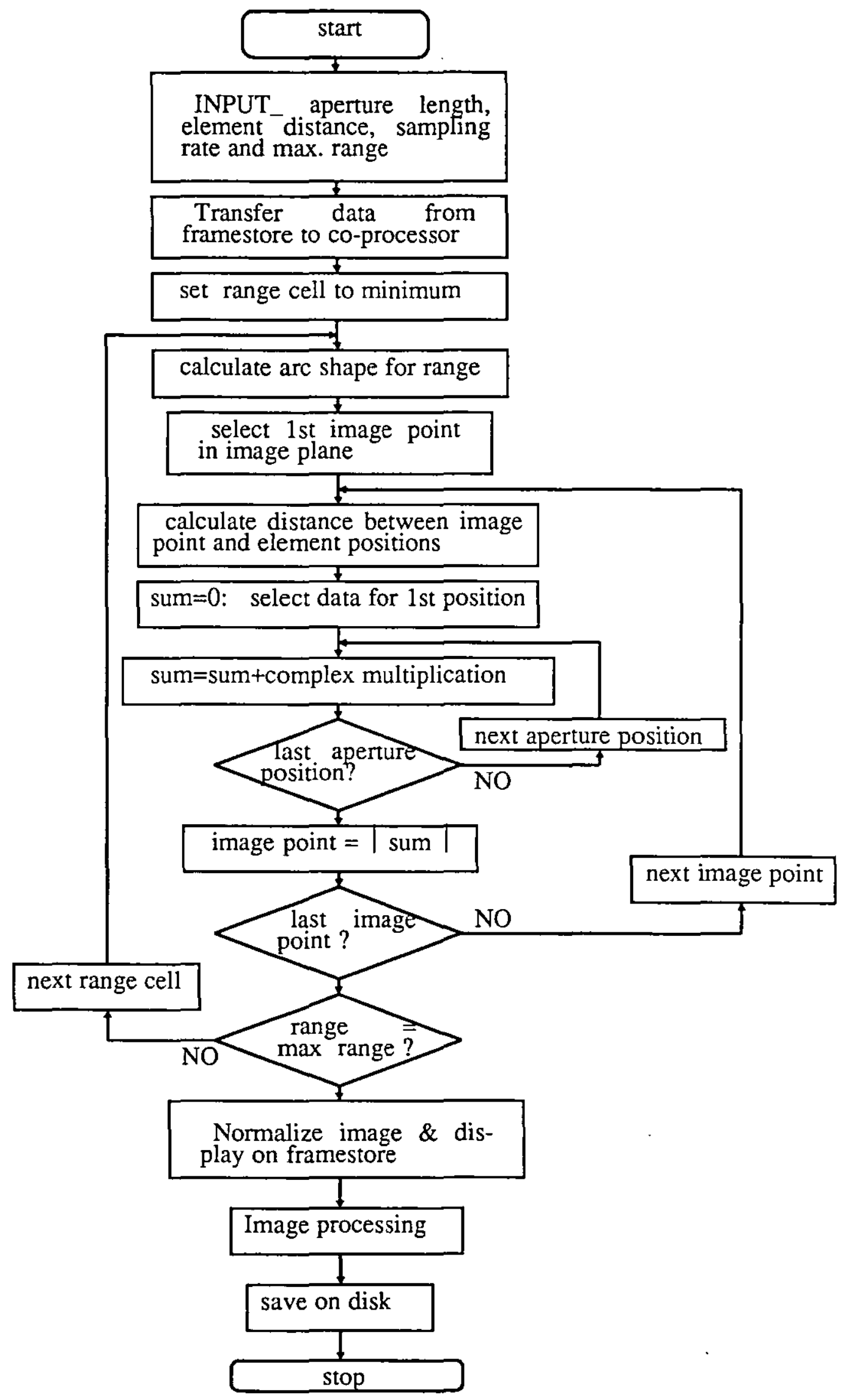

Fig. 6.12 Flowchart of the Image Reconstruction Procedure 


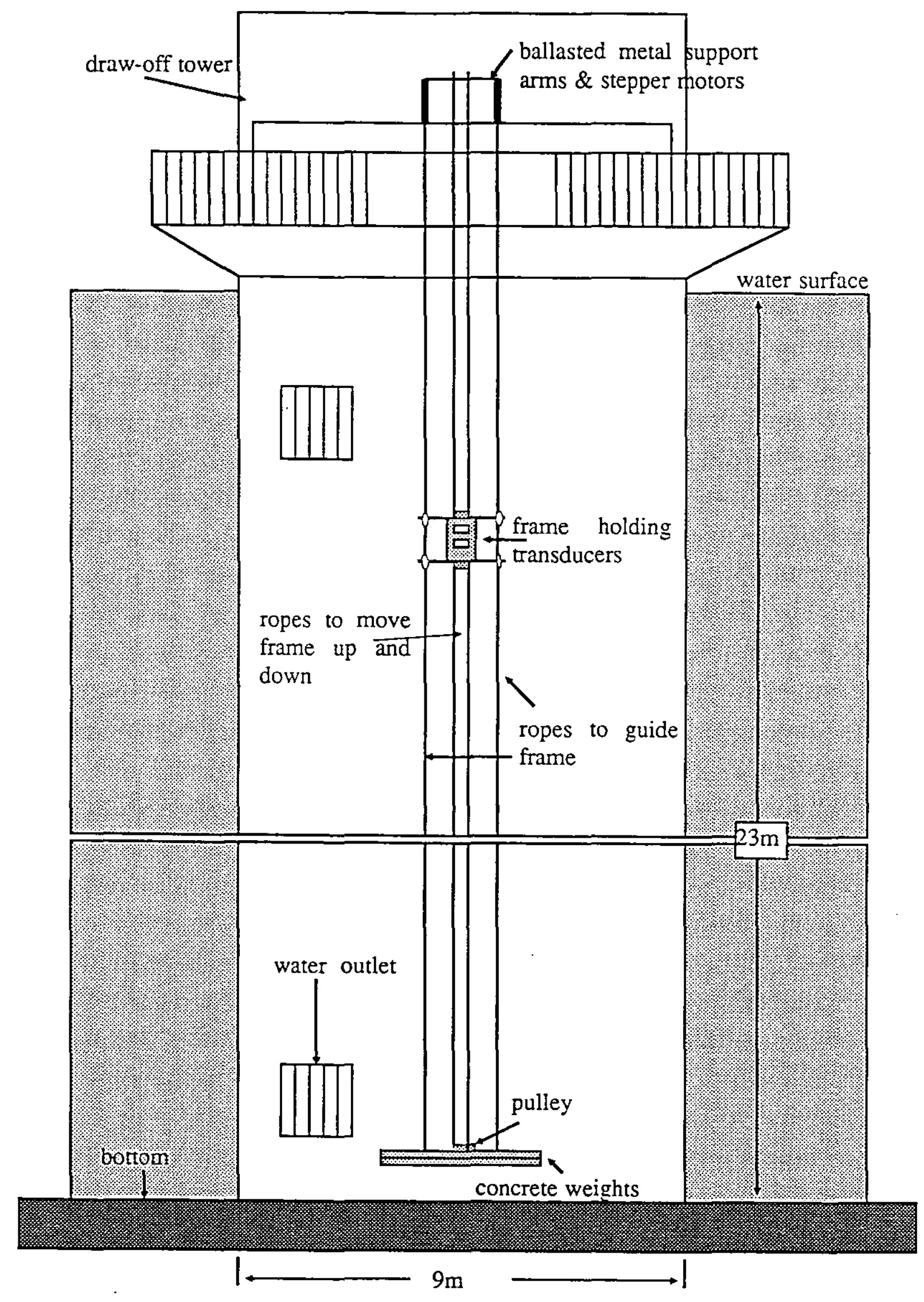

Fig, 6.13 Practical Setup of the Synthetic Aperture at the Reservoir. 
pulley wheel. A small pulley is fixed to the concrete weights which suspend just above the reservoir bottom and a rope is used to form a type of 'endless loop' using the small pulley and the large pulley at the top of the draw-off tower. The stepper motors drive the large pulley which in turn forces the rope to rotate in the endless loop and thus moving the frame holding the transducers up or down. Fig. 6.14 1 llustrates the arrangement of the stepper motors and the pulley wheel, both of which are fixed on to the weighted metal frame.

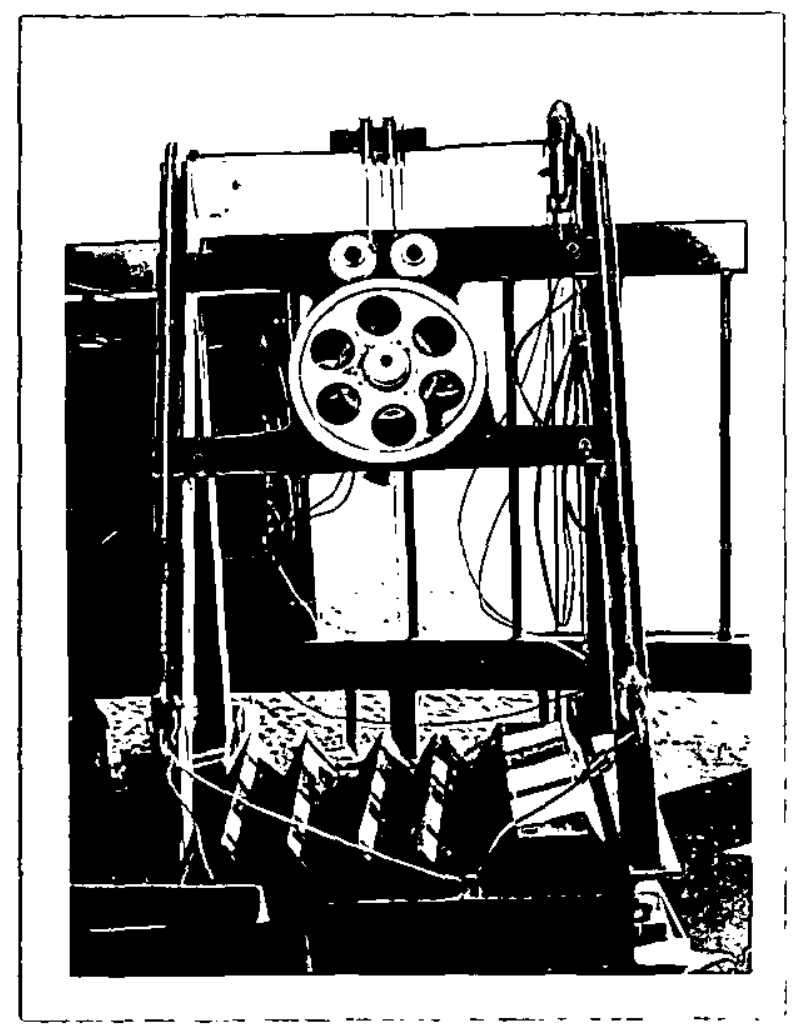

Fig. 6.14. Photograph of the Weighted Support Arms and the Stepper Motors used at the Reseryoir. 


\section{RESULTS}

\section{Z1 Introduction}

The results obtained using the synthetic aperture sonar system are presented in this chapter. showing both the resolution capabilities and the improvements due to transducer error motion correction and image processing techniques. The basic system comprising the BBC microcomputer, the co-processor, the framestore and the rack for the hardware is illustrated in Fig. 7.1. The first section contains the images obtained in the early experiments using the $150 \mathrm{kHz}$ and the $40 \mathrm{kHz}$ systems in the department tank during the development of the system. The remainder of the results are those obtained at a local reservoir with transducer error motion correction and image processing techniques to enhance the images.

The reconstructed images and results obtained using the system are presented in two different forms: as a colour hard copy. using an inkjet colour printer. providing a 2-D representation of the area under consideration and as an intensity modulated graph of image point amplitude for all image points lying at a particular range cell or interval. The second form can be considered as a cross-section taken. across the two dimensional image, parallel to the aperture.

These two representations are used to consider the different aspects of the reconstructed image. The colour 
printout uses a 2 by 2 dot motrix of 4 colours to represent 16 levels of image point amplitude. The plot of amplitude of an image point against cross-range position for a particular range cell provides the opportunity of studying the targets reconstructed more closely. measuring resolution and comparing amplitudes of a group of targets.

In the majority of cases the targets comprised air filled spheres of diameter $4 \mathrm{~mm}$ and $11 \mathrm{~mm}$ tied to a weight on the bottom to suspend at the desired depth. Figure 7.2 is a photograph of the targets being deployed in the department tank.

\subsection{Experiments in the Deportment Tank}

This section discusses and illustrates the results obtained in the department tank using the $150 \mathrm{kHz}$ and 40 $\mathrm{kHz}$ systems.

\subsection{Images Obtained using the $150 \mathrm{kHz}$ System}

The setup in the department tank laboratory has been described in section 6.2.7. The number of points in the aperture was chosen to be 256 points (for compatibility with storing data in the framestore), and with a sample spacing of $\lambda / 2$, an aperture of $128 \lambda$. i.e. $1.28 \mathrm{~m}$ was synthesized $(\lambda=0.01 \mathrm{~m})$. The minimum transmitted pulse of 100us. Fixed by the 0 of the transducers. Iimited the maximum obtainable range resolution to about $73 \mathrm{~mm}$. Two separate transducer arrays, each $1 \lambda$ by $6 \lambda$ in size were used, one as the transmitter and the other as the receiver. The targets were placed such as to be at mid-water depth and the transducers were mounted to provide a narrow vertical beamwidth and thus reduce multipath propagation due to the water surface and bottom. as the tank is only $2 \mathrm{~m}$ 


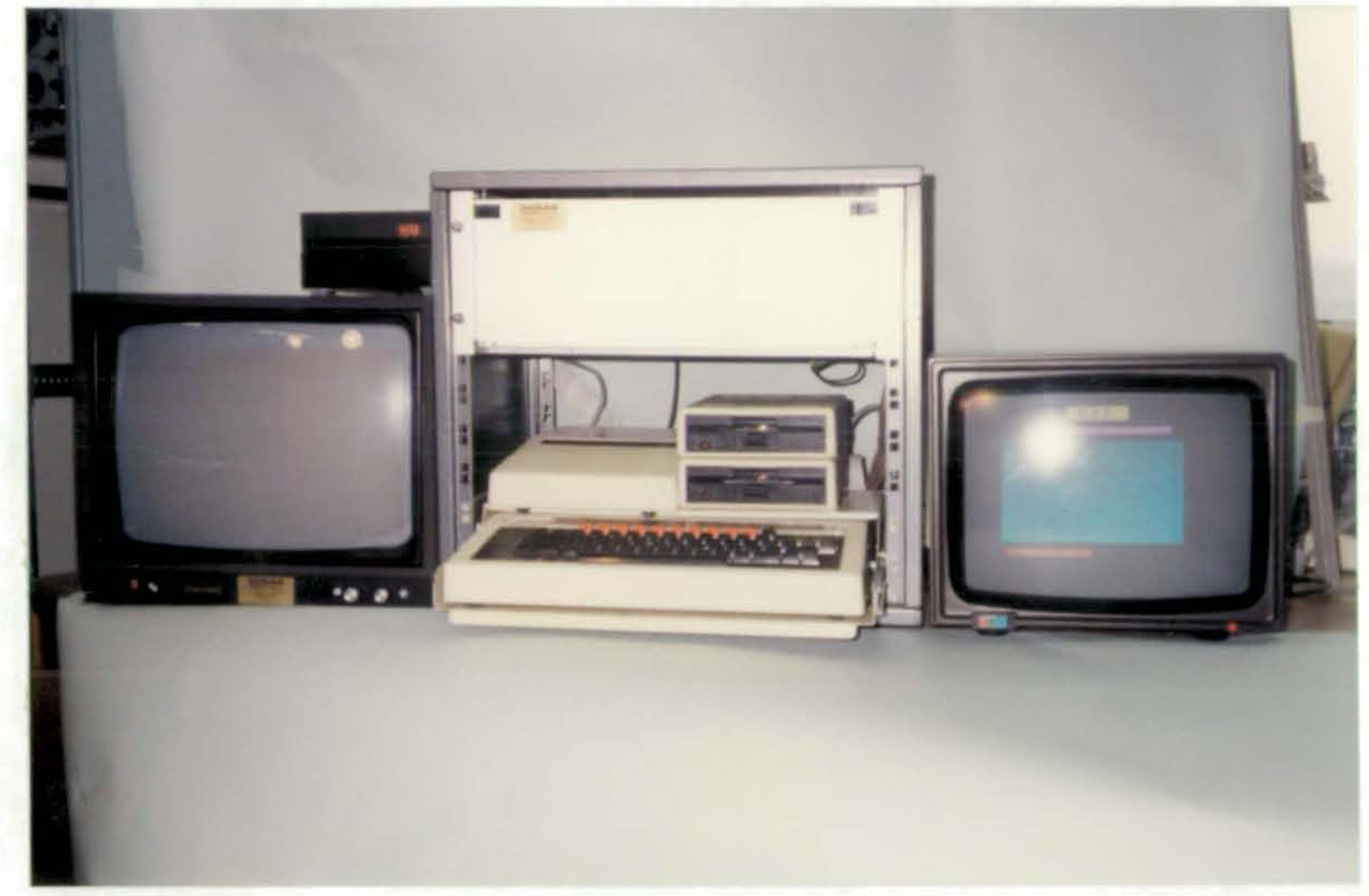

Fig. 7.1 A Photograph of the Basic S.A.S. System.

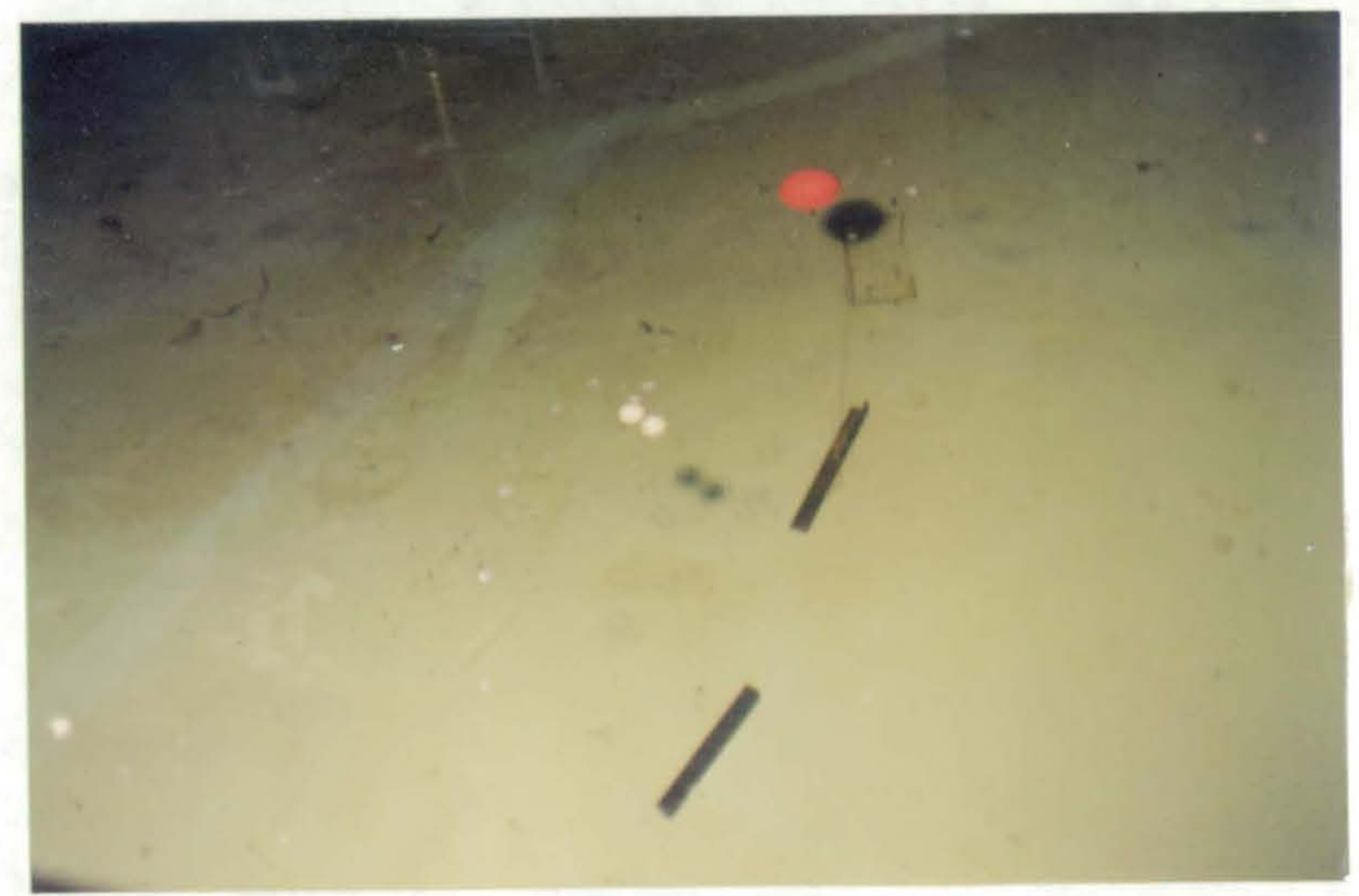

Fig, 7.2 A Photograph of the Targets being used in the Deapartment Tank 
deep. The imoges shown in this section have a maximum range of approximately $\mathrm{Sm}$ and a cross-range distance of 1. $28 \mathrm{~m}$.

Figure $7.3(b)$ IIlustrates the data expected due to the simulation of a single target at a range of $1.7 \mathrm{~m}$. shown in Fig. $7.3(a)$. The data due to the single target is shown to be lying in a hyperbolic arc in the data array and is caused by the variation in the distance between the target and the transducer as the transducer moves along the aperture and has been discussed in section 3.3. Figure $7.3(c)$ shows the image resulting from the reconstruction of the simulated data with a single point target.

The data and results obtained from a single target in the department tank at a range of $1.7 \mathrm{~m}$ is shown in $\mathrm{Fig}$. 7.4. The data due to the target can be clearly seen. in Fig. 7.4(b) to be lying in a hyperbolic arc in the data array as expected. The amplitude of variation of the data, however, decreases as the transducer moves to either side of the target and is due to the effects of the transducer beam pattern. When the target is normal to the transducer position there is a strong echo from the target. but as the transducer moves to either side, the target 'moves' out of the main beam of the transducer and thus the amplitude of the echo decreases. The far wall of the tank at a range of $8.5 \mathrm{~m}$, is reconstructed and there is the presence of low level noise due to reverberation and multipath propagation from the tank sides, surface and bottom. The reconstructed image compares favourably with the simulated Image obtained in fig. 7.3(c).

To measure the cross-range resolution of the system. we can display image amplitude versus cross-range (or aperture position) along a particular range cell. 


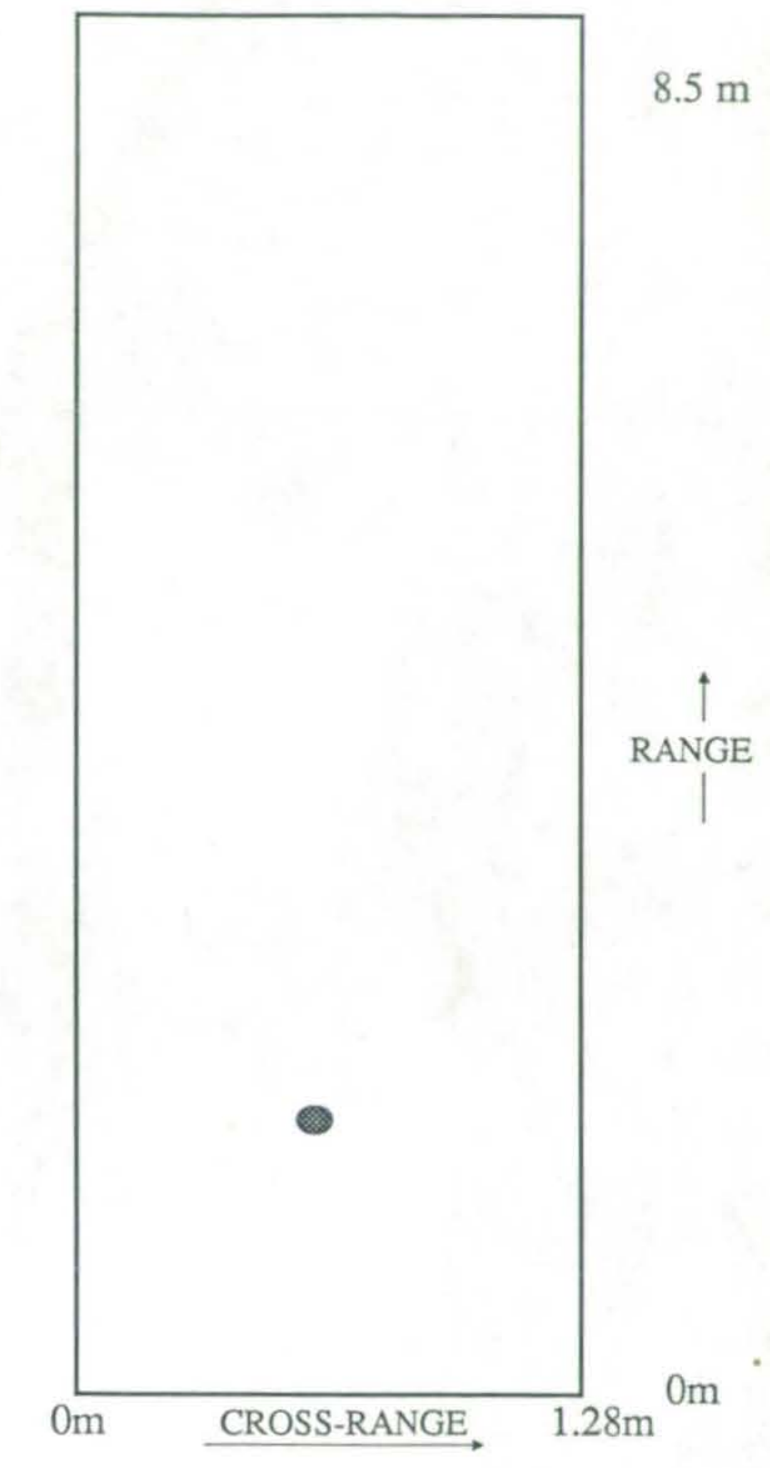

Fig, 7.3(a) Target Configuration

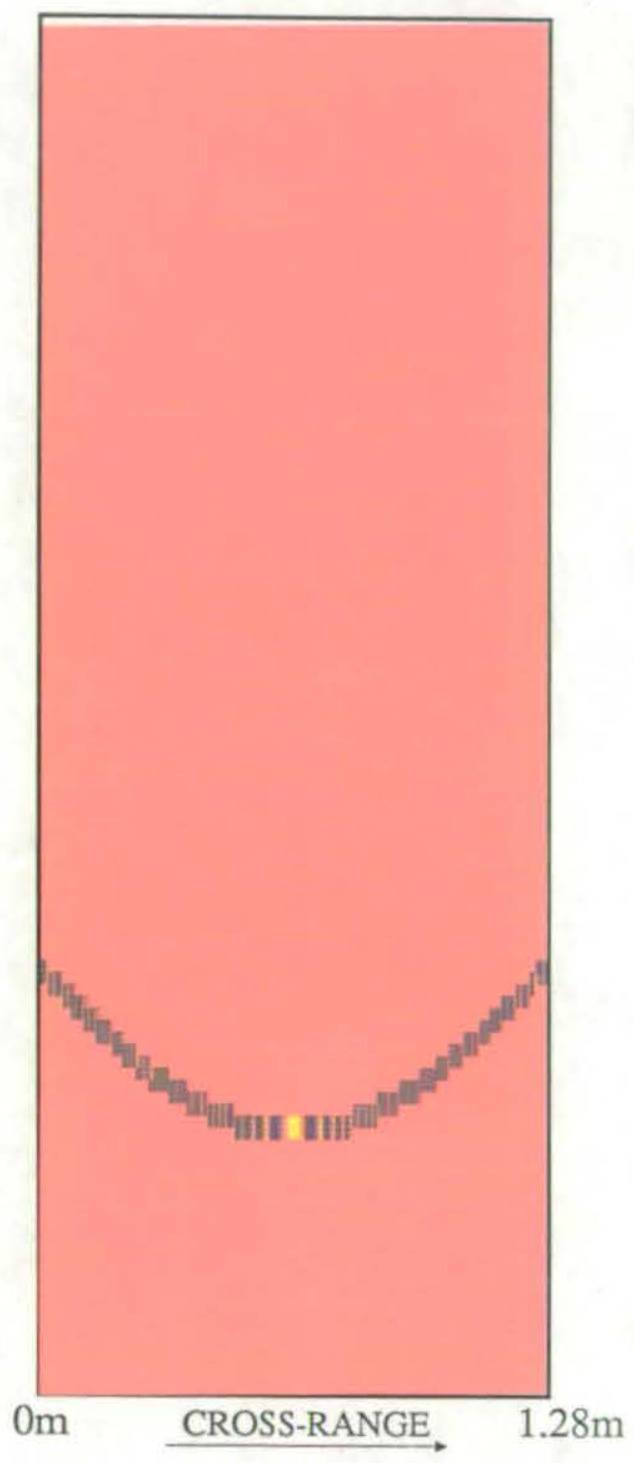

Fig, 7.3(b) Simulated Data of the Target Configuration shown in Fig. 7.3(a).

Fig, 7.3 Simulated Results 


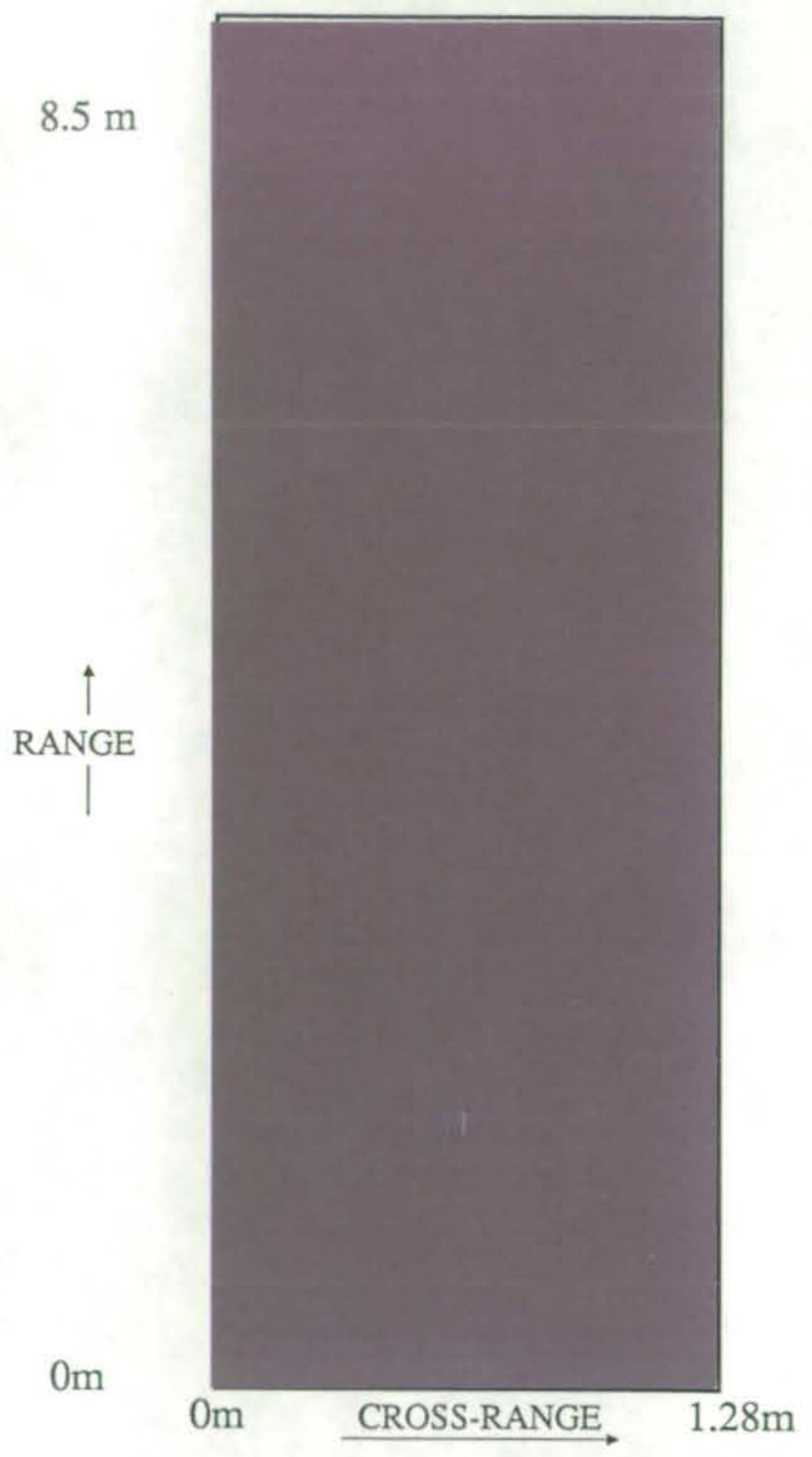

Fig. 7.3(c) Reconstructed Image of the Simulated Target Configuration Shown in Fig, 7.3(a). 


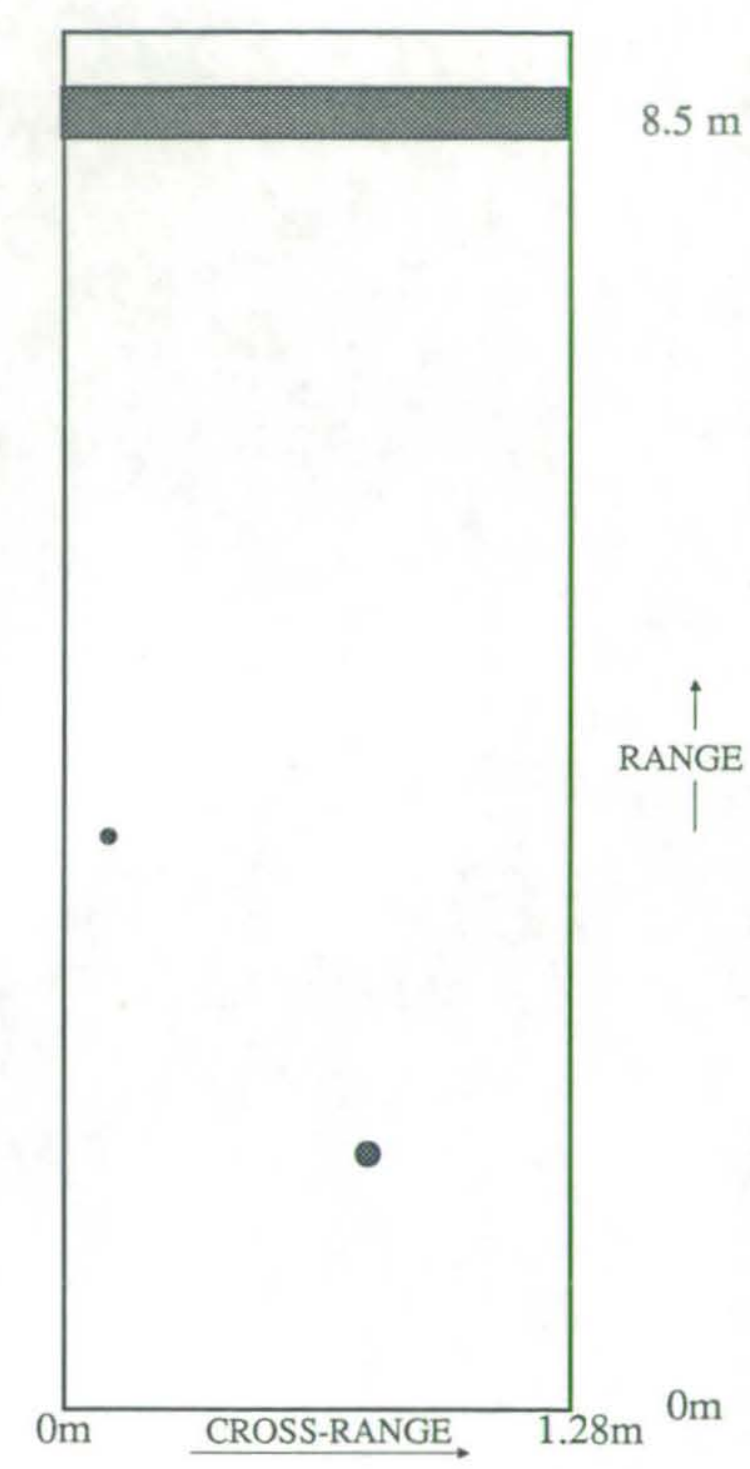

Fig. 7.4(a) Target Configuration

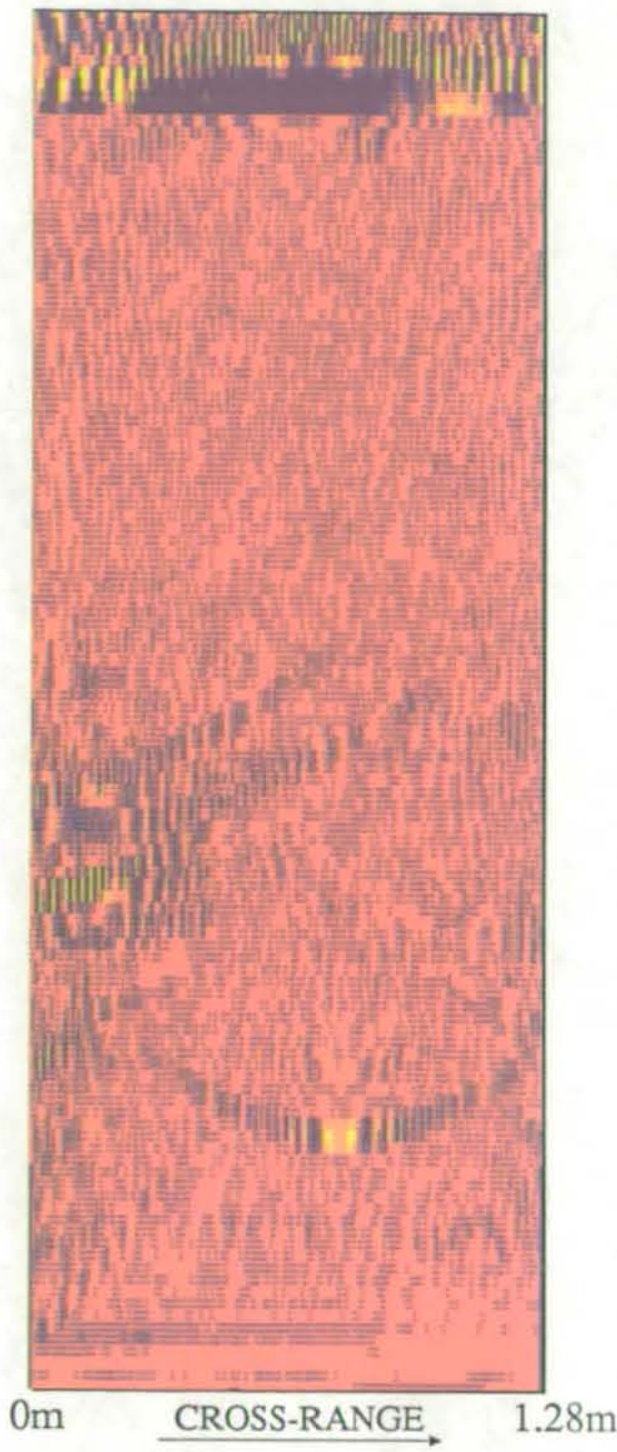

Fig. 7.4(b) Collected Data from the Target Configuration shown in Fig. 7.4(a).

Fig. 7.4 Results from the Tank. 


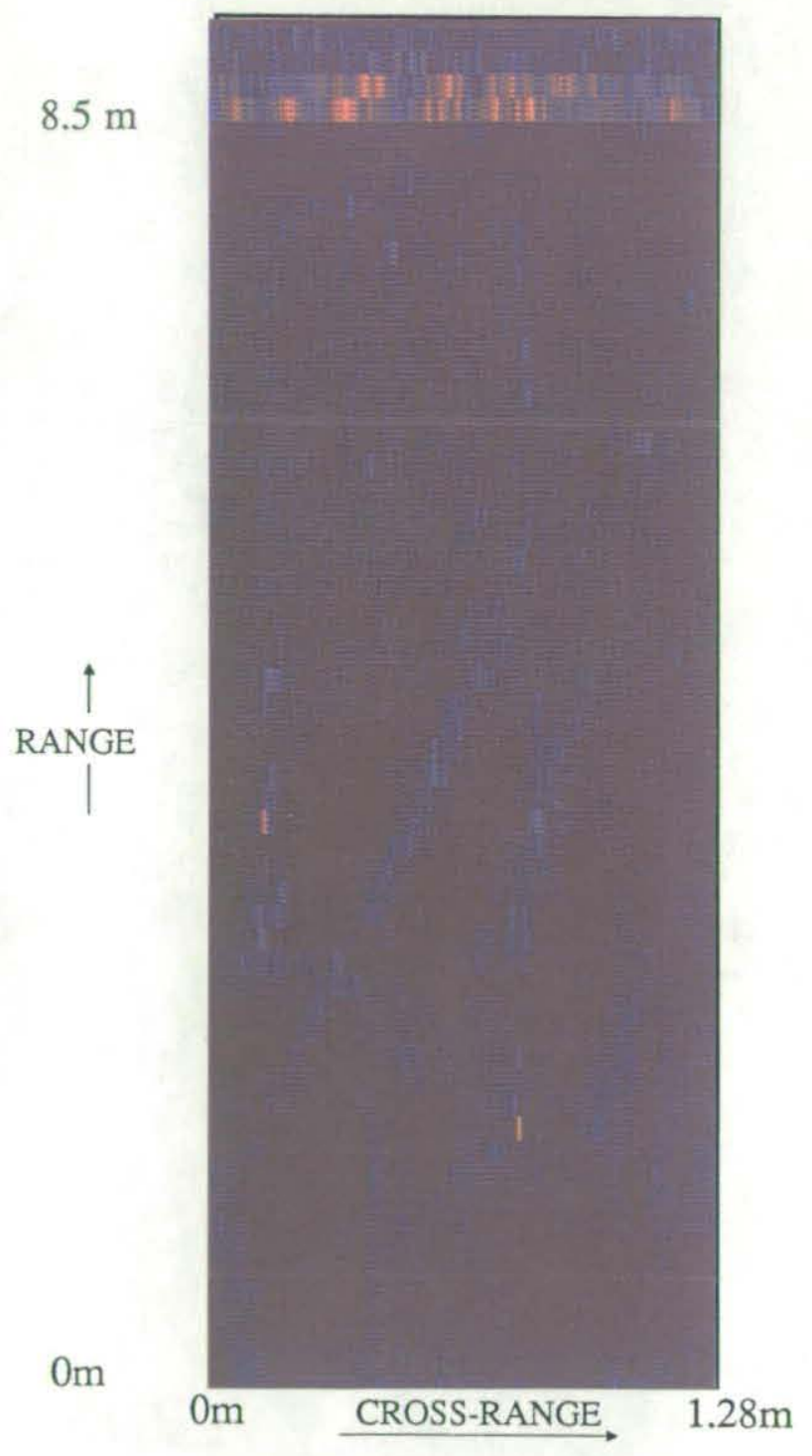

Fig. 7.4(c) Reconstructed Image of the Target Configuration Shown in Fig. 7.4(a). 
Consider a single target at a range of $1.5 \mathrm{~m}$ shown in Fig. $7.5(a)$. The reconstructed image obtained from the target configuration is shown in Fig. 7.5(b). A sampling frequency of $20 \mathrm{kHz}$ makes each range cell $7.3 \mathrm{~cm}$ wide, and the target at a range of $1.5 \mathrm{~m}$ lies in range cell number 20. A plot of image amplitude along range cell number 20 (parallel to the aperture) is shown in Fig. 7.6. The expected cross-range resolution from a non-optimised focussed synthetic aperture system has been discussed in section 2.6 .2 and is equal to $d_{*} / 2 D$ where $d$ is the transducer width, $D_{\diamond}$ is the optimum aperture length and $D$ is the actual aperture, in a single moving transducer system. Hence with a transducer of width $10 \mathrm{~mm}$ and an optimum aperture length of $1.5 \mathrm{~m}$ the expected lateral resolution is equal to $6 \mathrm{~mm}$. Measuring the value of cross-range resolution from the plot in Fig. 7.6 gives a value of $6 \mathrm{~mm}$ which is equal to the expected value.

Further examples of target configuration and their reconstructed images are shown in Figs. $7.7,7.8$ and 7.9 .

\subsubsection{Images Obtained using the $40 \mathrm{kHz}$ System}

The results and images obtained using the $40 \mathrm{kHz}$ system in the department tank are discussed in this section. The operating frequency was reduced to $40 \mathrm{kHz}$ to make the system more resilient to transducer motion errors with the larger wavelength at $40 \mathrm{kHz}$ and also to enable the system to operate to larger ranges with the reduction in the absorption co-efficient at a lower frequency.

The transducer arrays comprised 4 circular elements in a row and with $\lambda=37.5 \mathrm{~mm}$ this resulted in an array size of $37.5 \mathrm{~mm}$ by $150 \mathrm{~mm}$. The transmit pulse width was 1 imited by the $Q$ of the transducers to a minimum of 200us. At an 


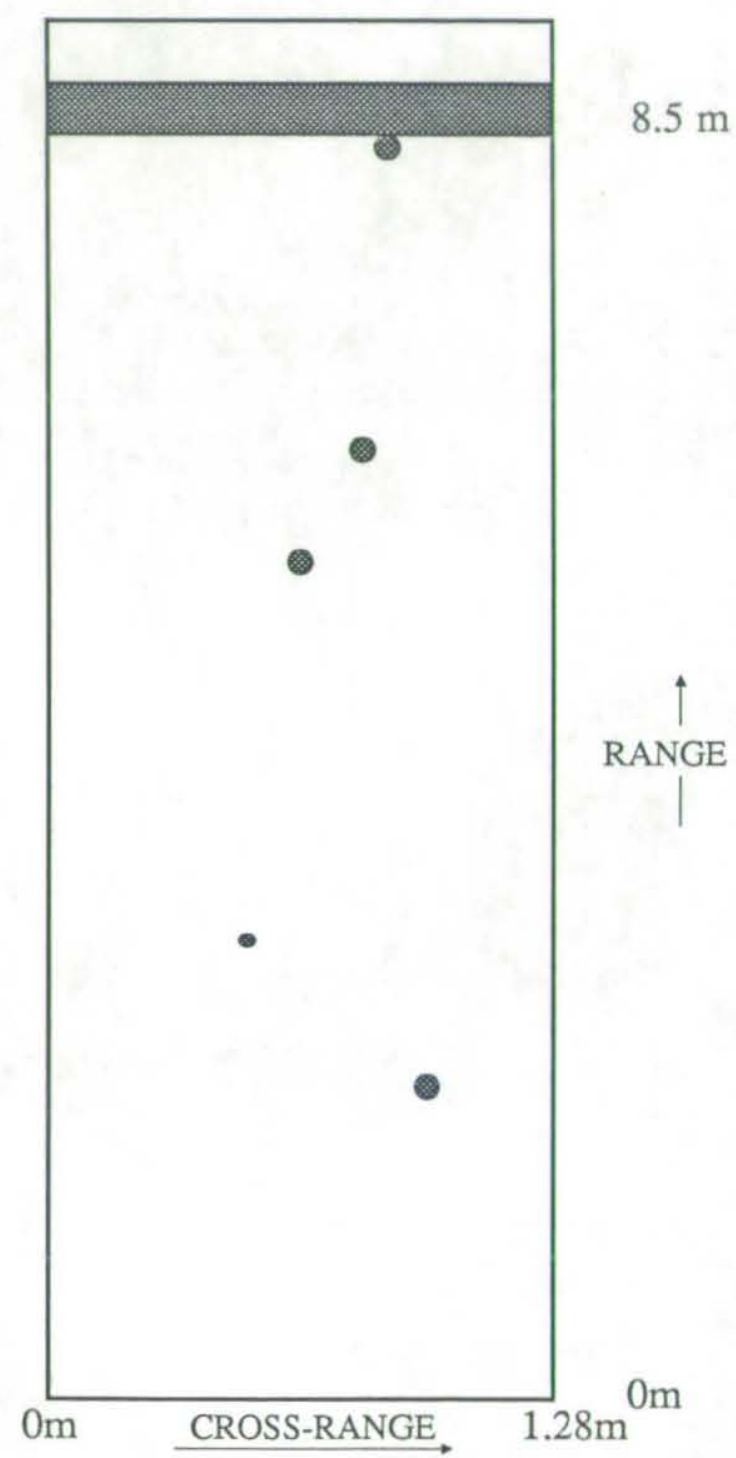

Fig. 7.5(a) Target Configuration

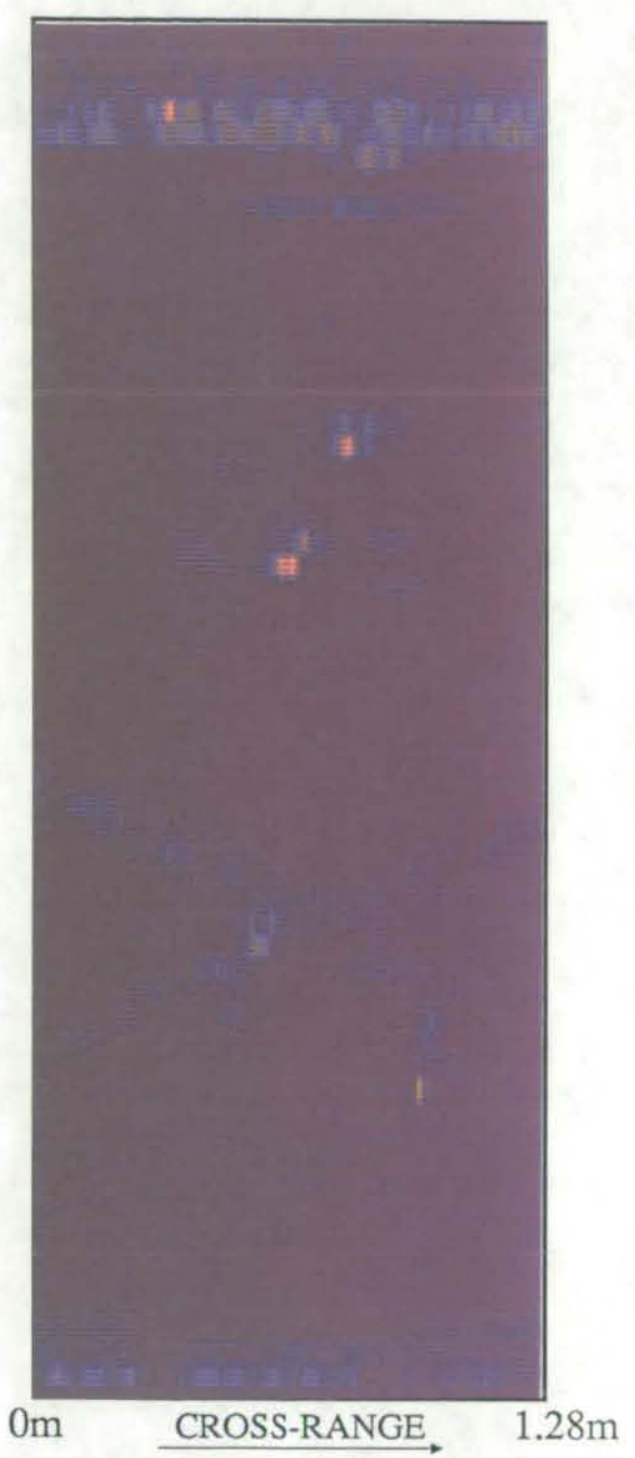

Fig, 7.5(b) Reconstructed Image of the Target Configuration shown in Fig, 7.5(a).

Fig, 7.5 Measured Data Reconstruction 


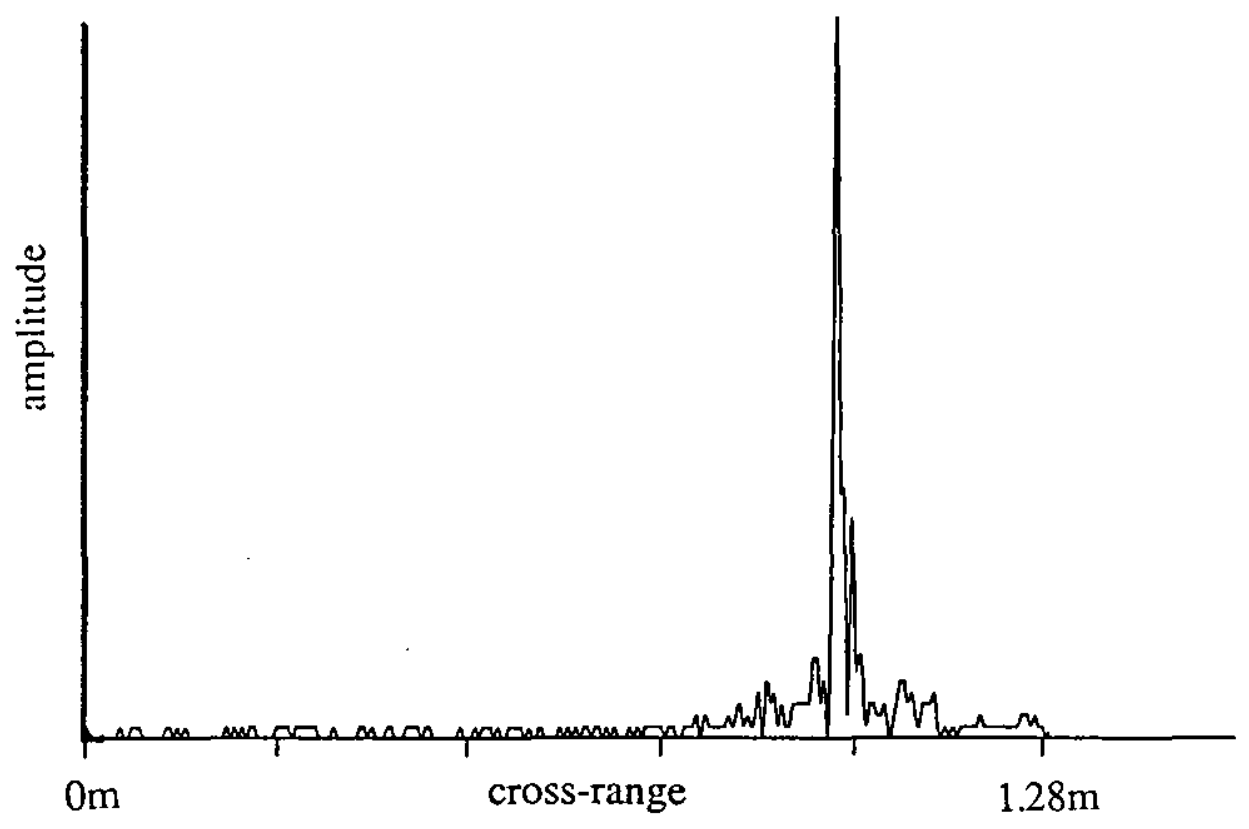

Fig. 7.6(a) Image Amplitude Variation across Range Cell 20 of Fig. 7.5(b)

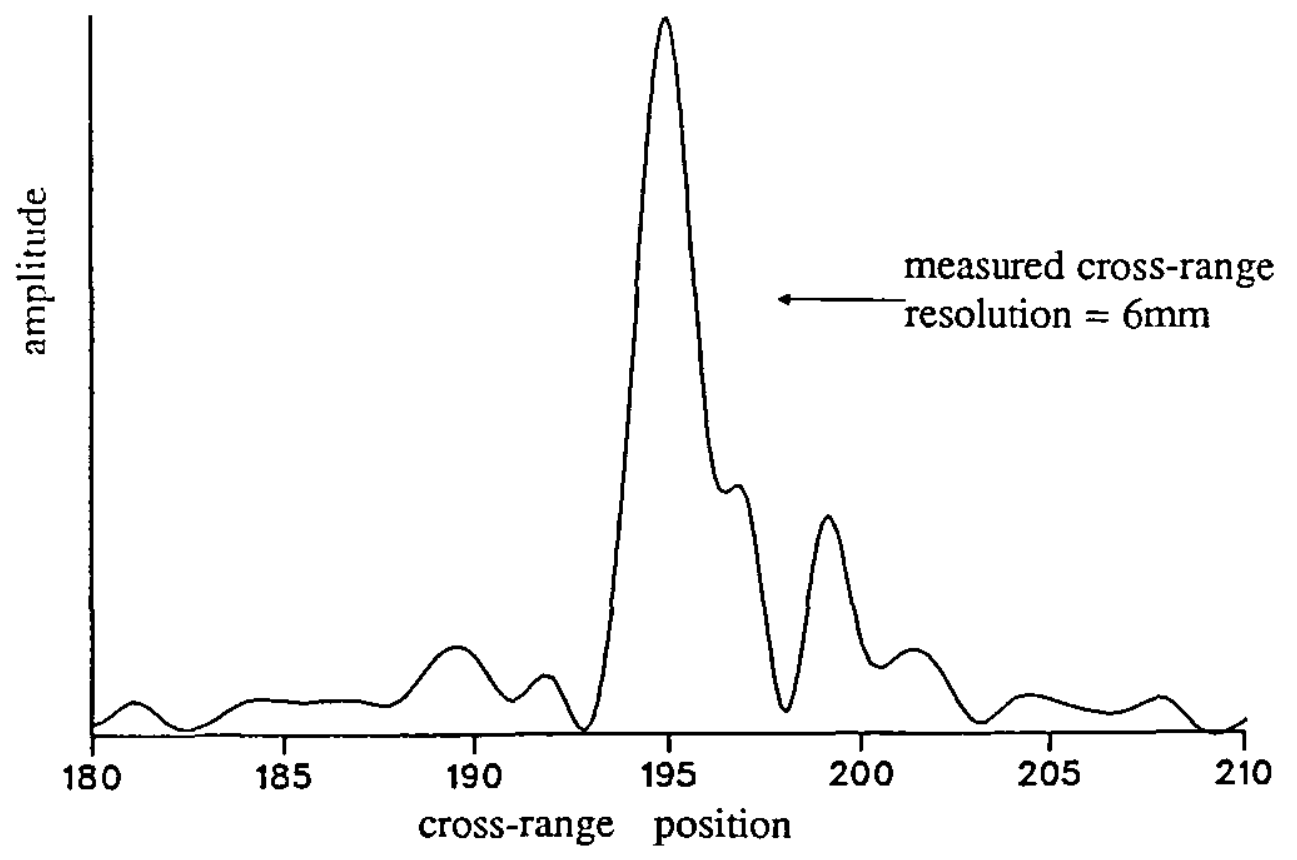

Fig. 7.6(b) Expanded Plot of Fig. 7.6(a) to Measure the Cross-Range Resolution. 


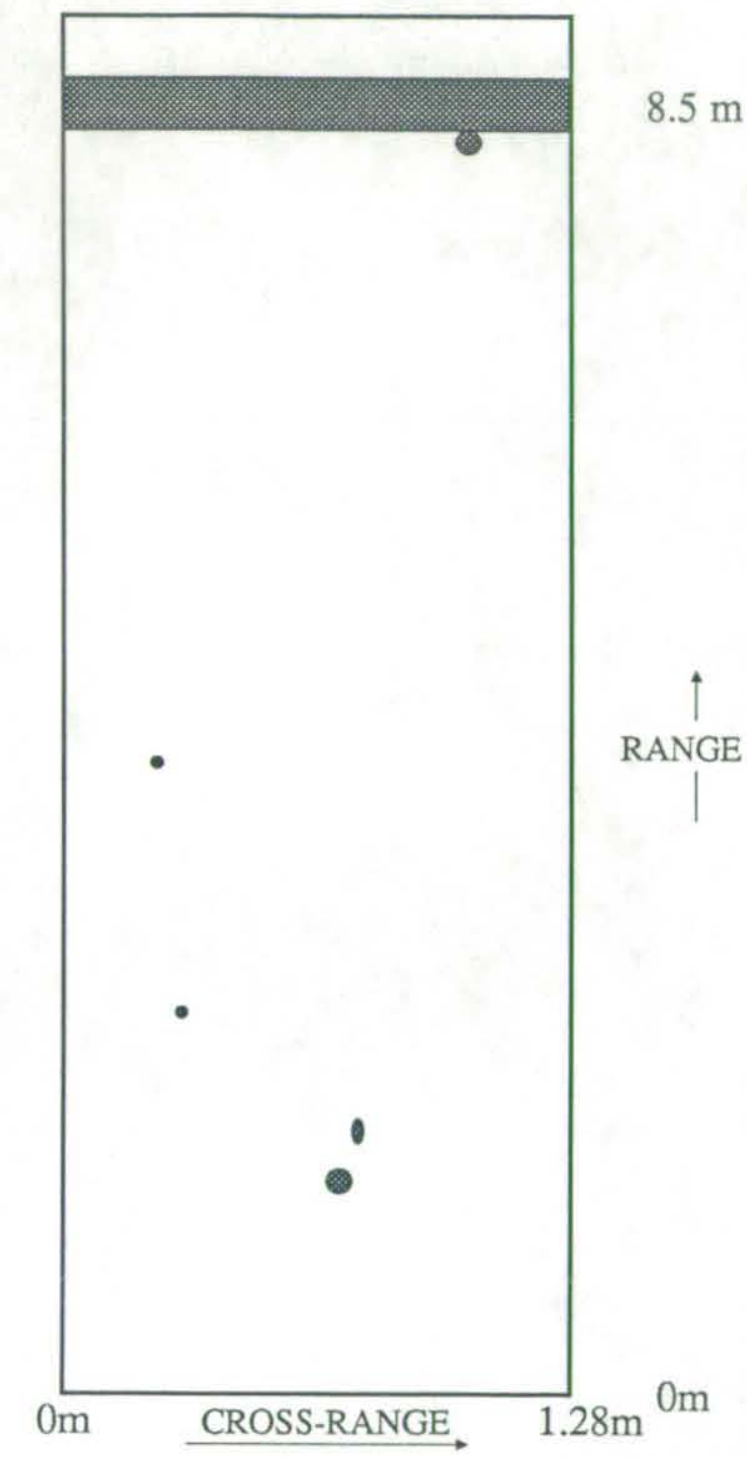

Fig. 7.7(a) Target Configuration

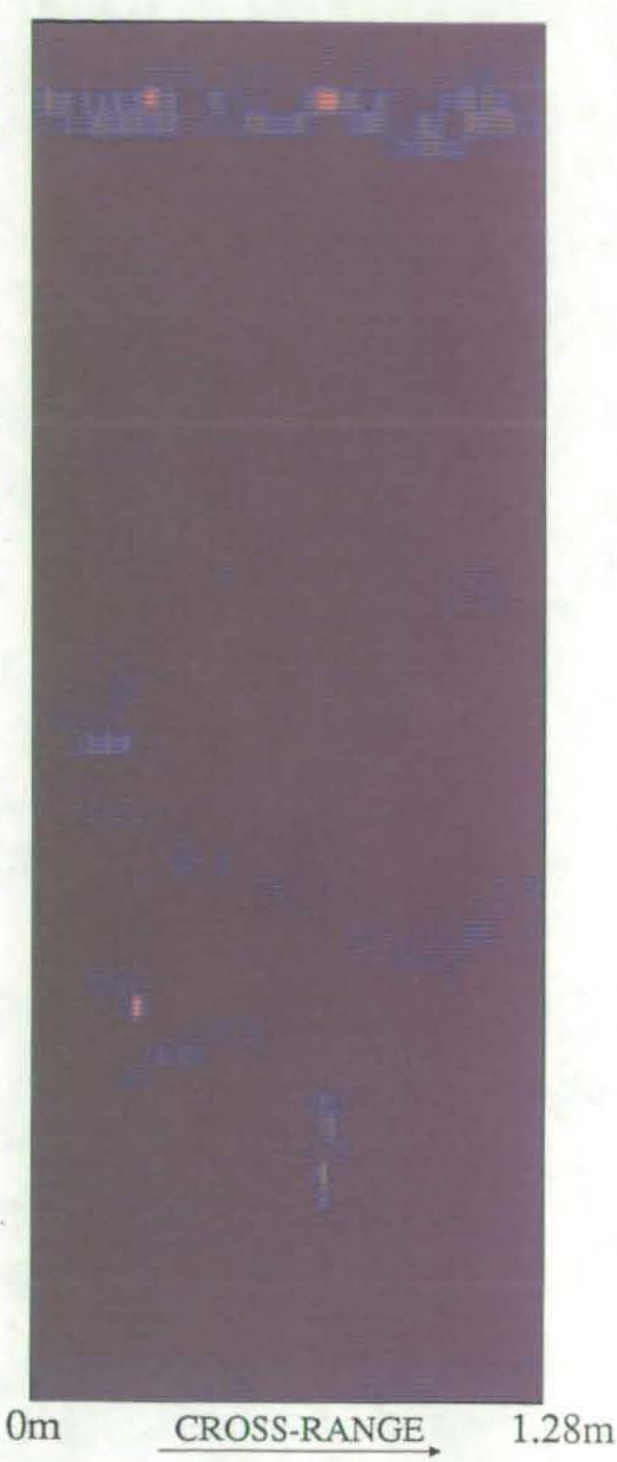

Fig. 7.7(b) Reconstructed Image of the Target Configuration shown in Fig. 7.7(a).

Fig, 7.7 Reconstruction using Collected Data 


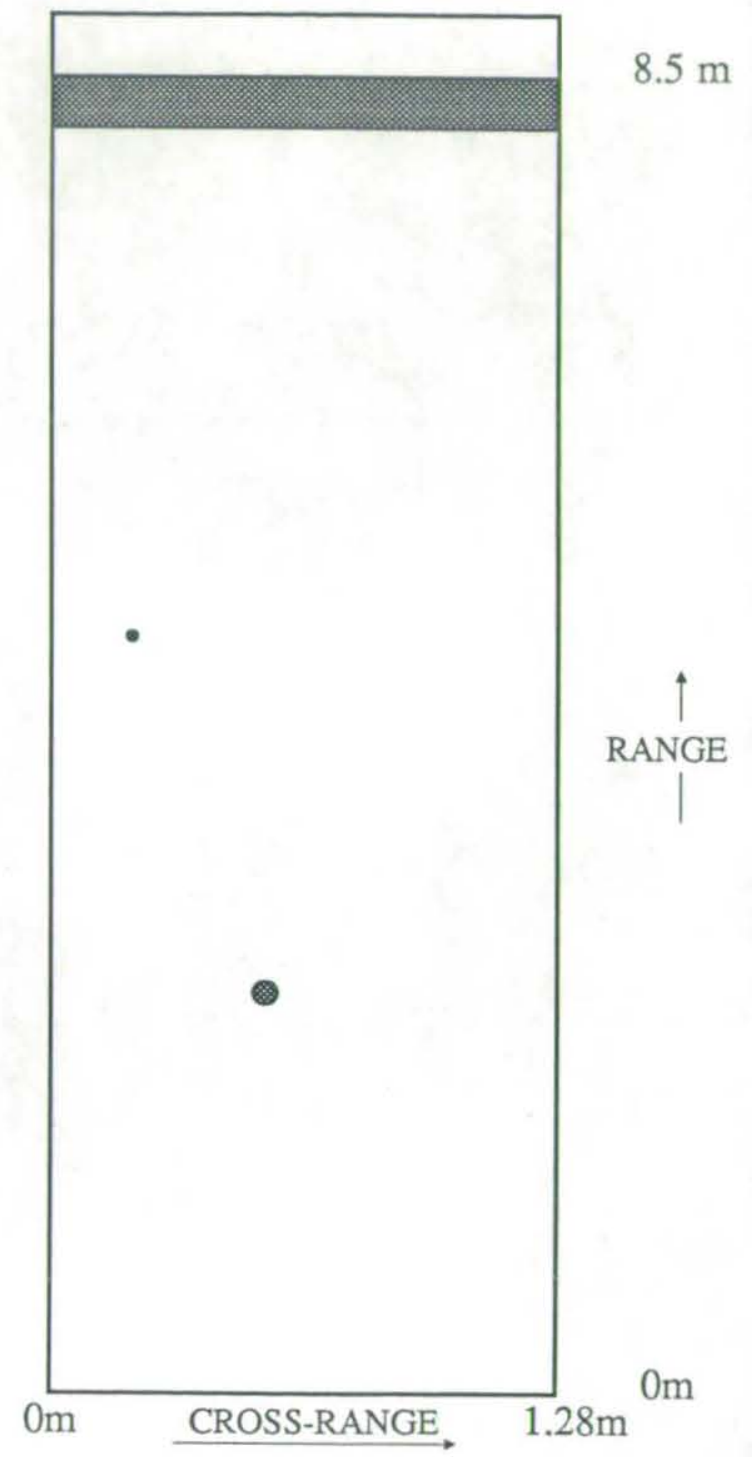

Fig. 7.8(a) Target Configuration

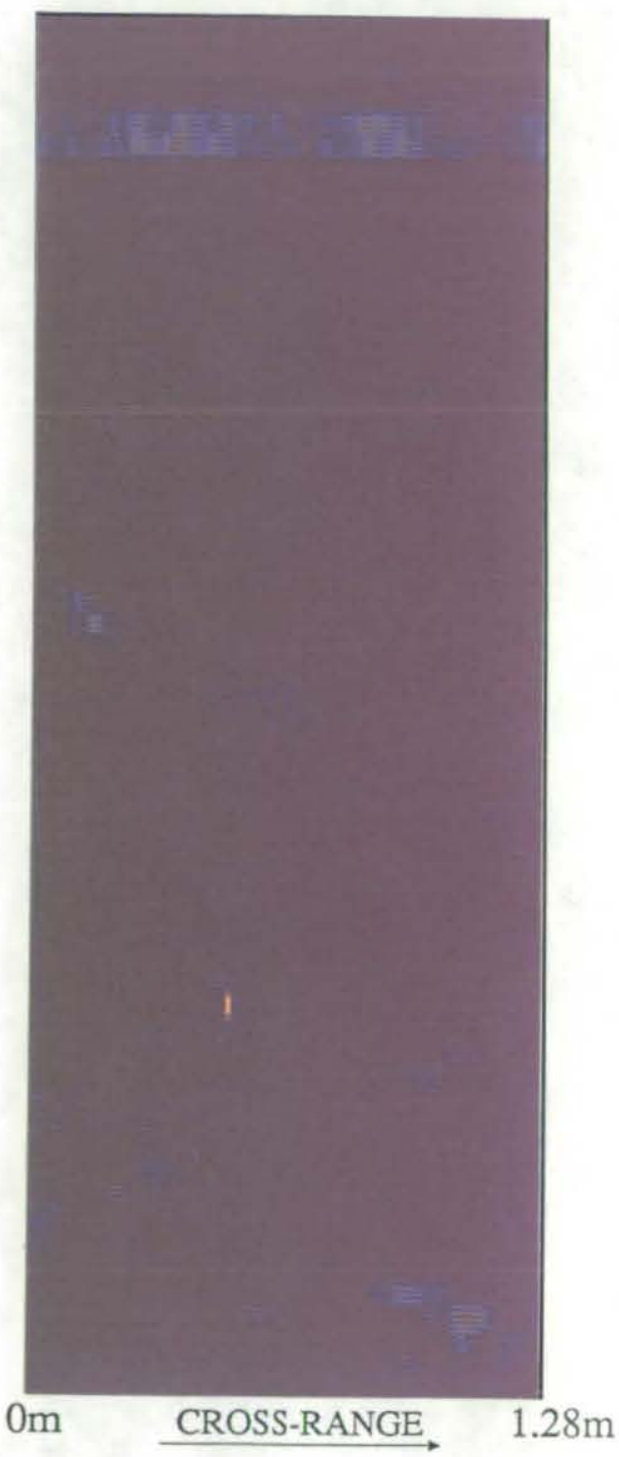

Fig, 7.8(b) Reconstructed Image of the Target Configuration shown in Fig, 7.8(a).

Fig. 7.8 Reconstruction using Collected Data 


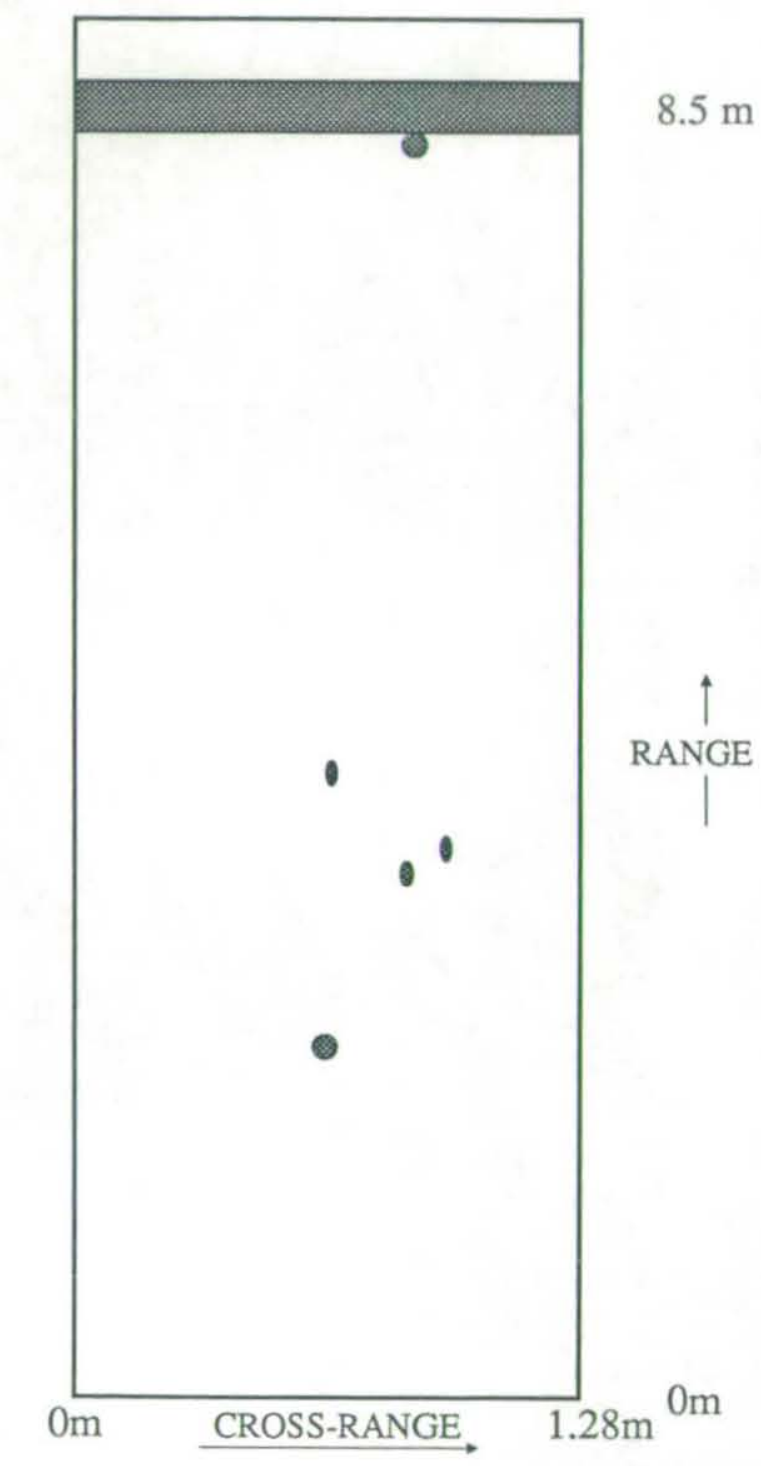

Fig. 7.9(a) Target Configuration

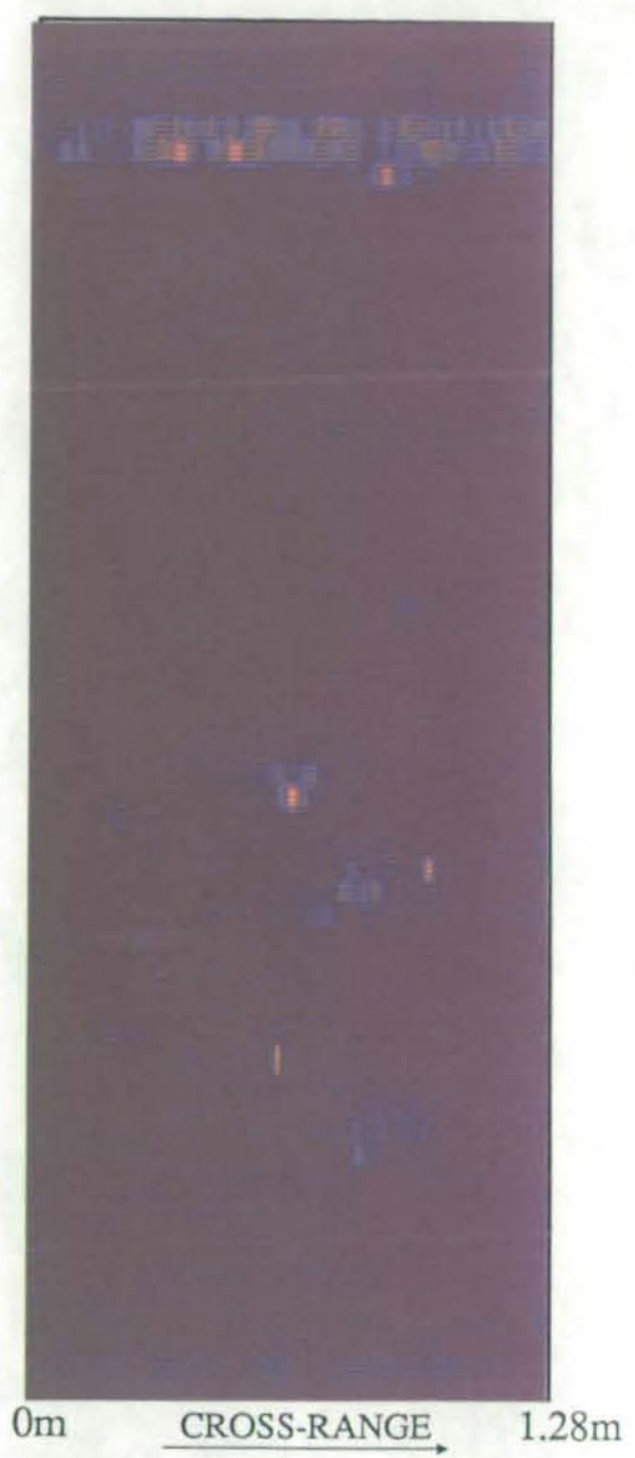

Fig, 7.9(b) Reconstructed Image of the Target Configuration shown in Fig. 7.9(a).

Fig. 7.9 Reconstruction using Collected Data 
operating frequency $40 \mathrm{kHz}$, an aperture of $128 \lambda$ is equivalent to $4.572 \mathrm{~m}$ in length, and with a sampling frequency of $20 \mathrm{kHz}$ the range cell width $1 \mathrm{~s} 73 \mathrm{~mm}$. The images shown in this section have a maximum range of approximately $9 \mathrm{~m}$ and a cross-range distance of $4.672 \mathrm{~m}$.

Another use the plot of the lmage amplitude versus cross-range or aperture position is to compare the target strengths of a group of targets. Consider the target conflguration shown in f1g. $7.10(\mathrm{a})$ consisting of an atr filied sphere of approxlmate diameter $11 \mathrm{~cm}$ at a range of $.2 .3 \mathrm{~m}$ and two smalier spheres of diameter $4 \mathrm{~cm}$ with a geparation of $10 \mathrm{~cm}$ at a range of $1.5 \mathrm{~m}$. The larger target has a target strength of -31 dB and the smaller targets have target strengths of -40 dB (from egtn. [2.50]. The reconsiructed image for the target configuration shown in Fig. $7.10(\mathrm{a})$ is illustrated in F1g.7.10(b). Plots of Image amplitude versus cross-range position for the targets are shown in Fig. 7.11 and Fig. 7.12. The difference in Image amplitude vetween the larger target and the smaller targets is due to the difference in their target strengths and transmission loss due to spherical spreading (Jcsses due to attenuation can be ignored as distances involved are small). The two way transmission loss in the signal from the larger target to the array is approximately $\therefore-14.4 \mathrm{dBs}$ and to the smaller targets the loss is equal to -7 des. Thus if we take into consideration the effects of itarget strength and transmission loss due to spherical spreading the difference between the amplitudes of the targets should be about $1.6 \mathrm{~dB}$ which can be compared to the amplitude difference between the targets in Figs. 7.13 and 7.12. A reconstruction of the simulated jata of the target configuration shown in Fig. 7.1D(a) 18 shown in Fig. 7.13. Plots of lmage amplitude versus cross-range position of the simulated data reconstruction are shown in Fig. 7.14 , 


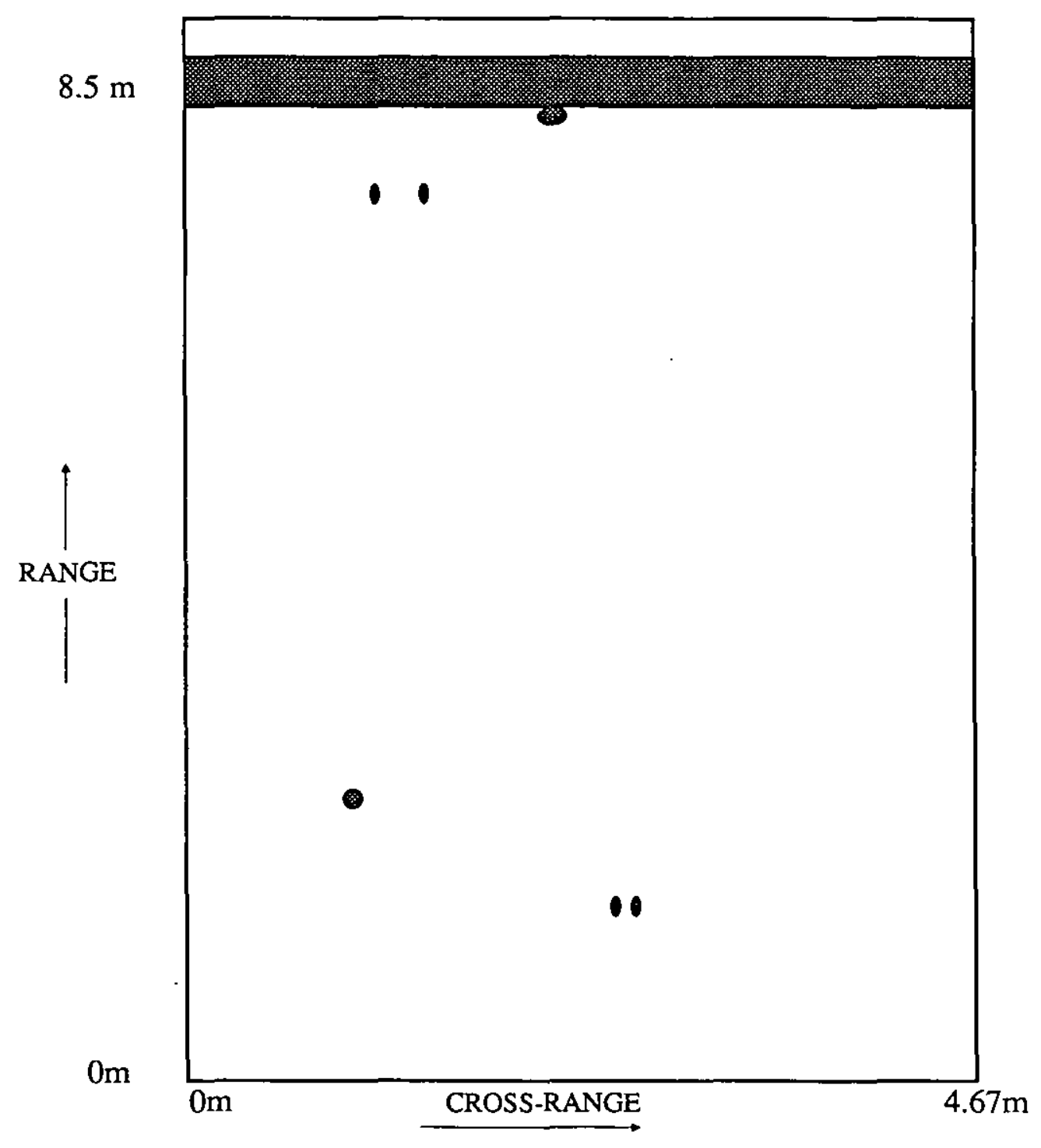

Fig, 7.10(a) Target Configuration 


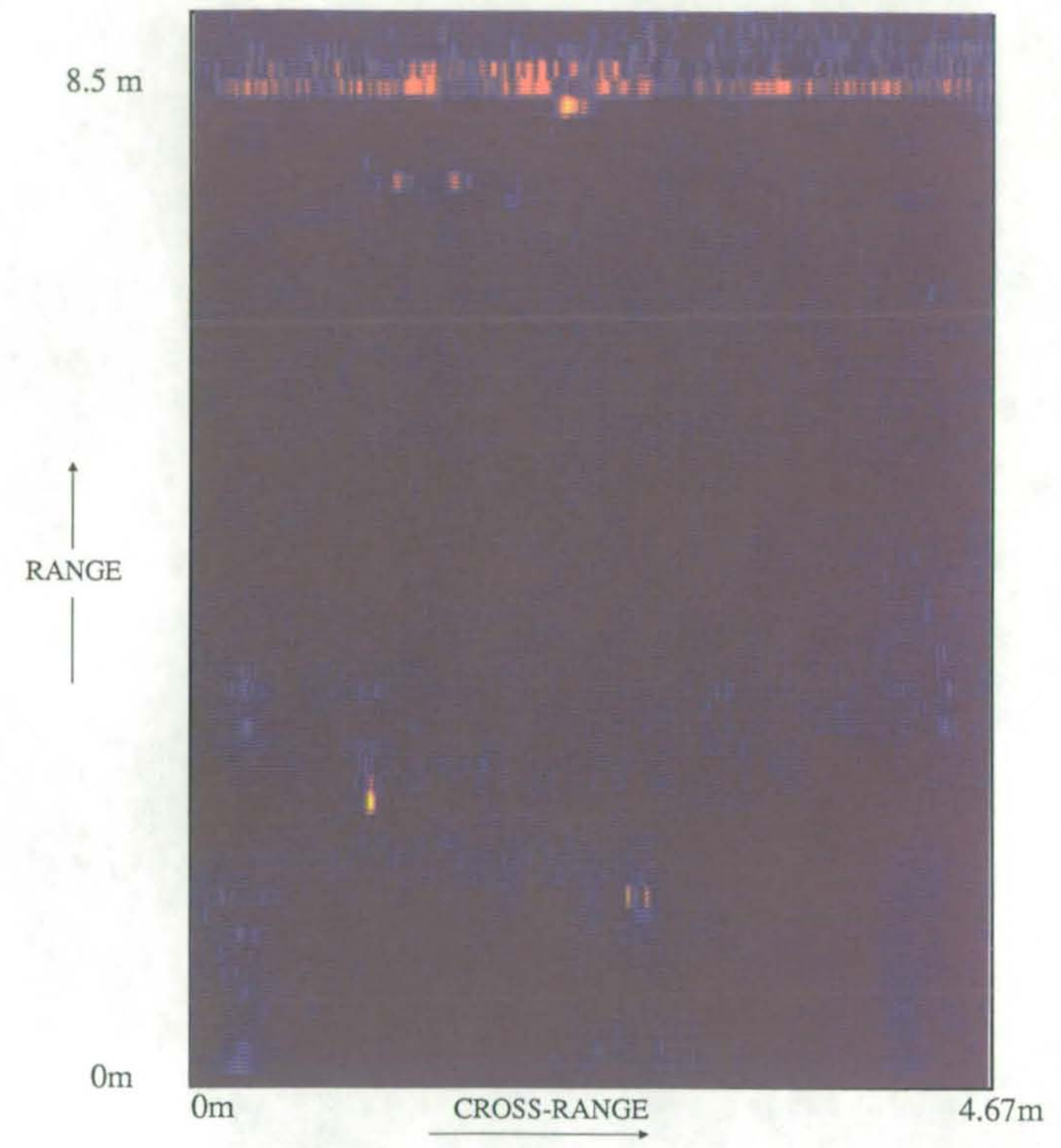

Fig. 7.10(b) Reconstructed Image of Target Configuration shown in Fig. 7.10(a). 


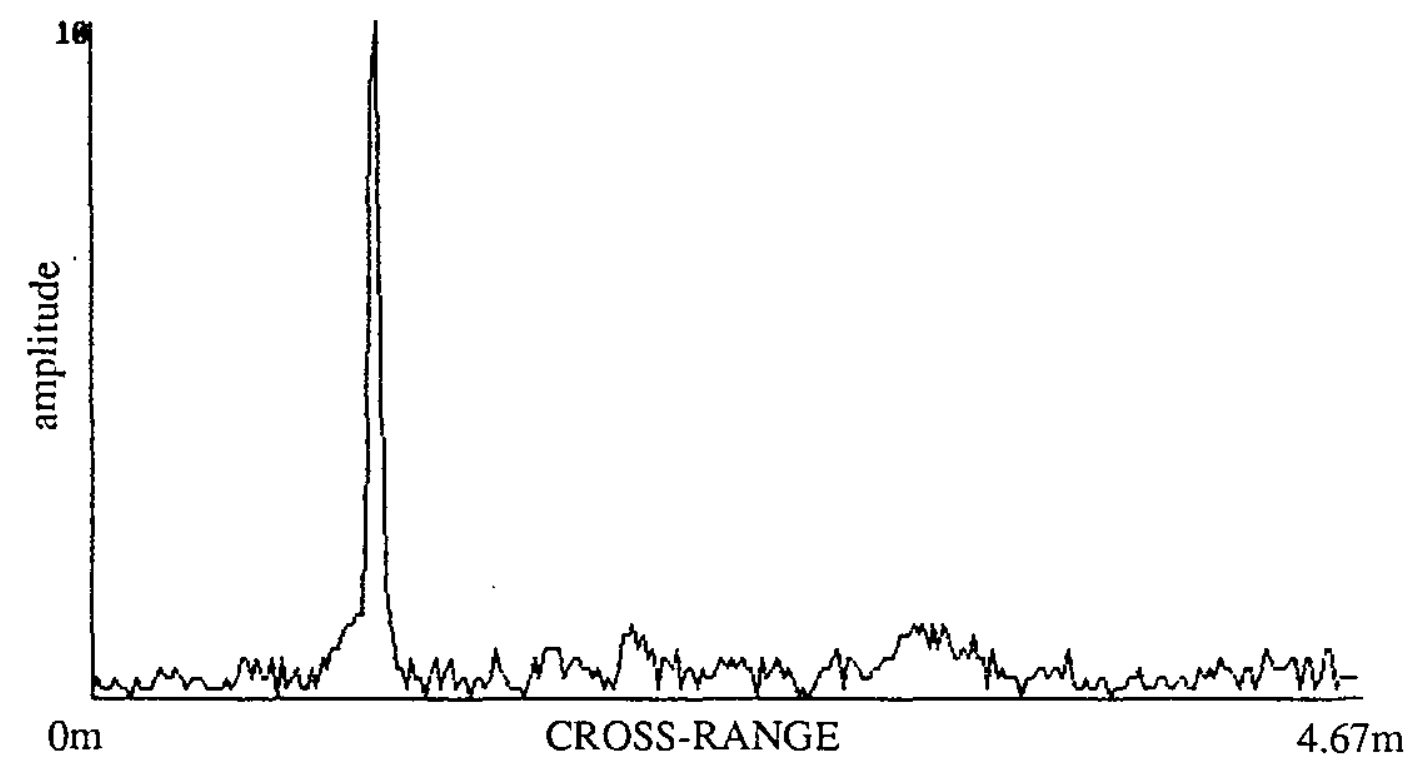

Fig. 7.11 Image Amplitude Variation across Range Cell 31 of Fig. $7.10(\mathrm{~b})$

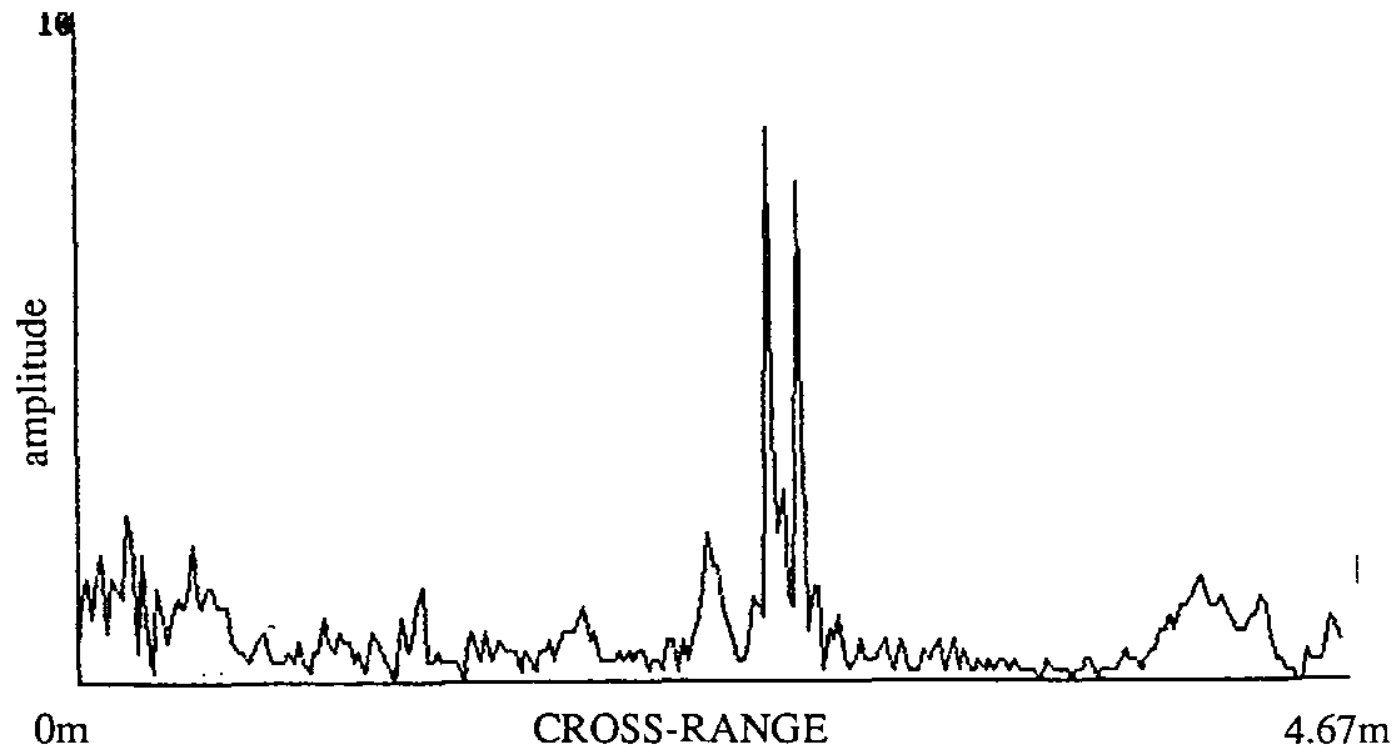

Fig. 7.12 Image Amplitude Variation across Range Cell 20 of Fig. 7.10(b) 
$8.5 \mathrm{~m}$

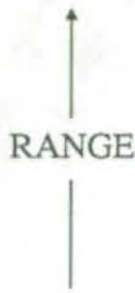

$0 \mathrm{~m}$

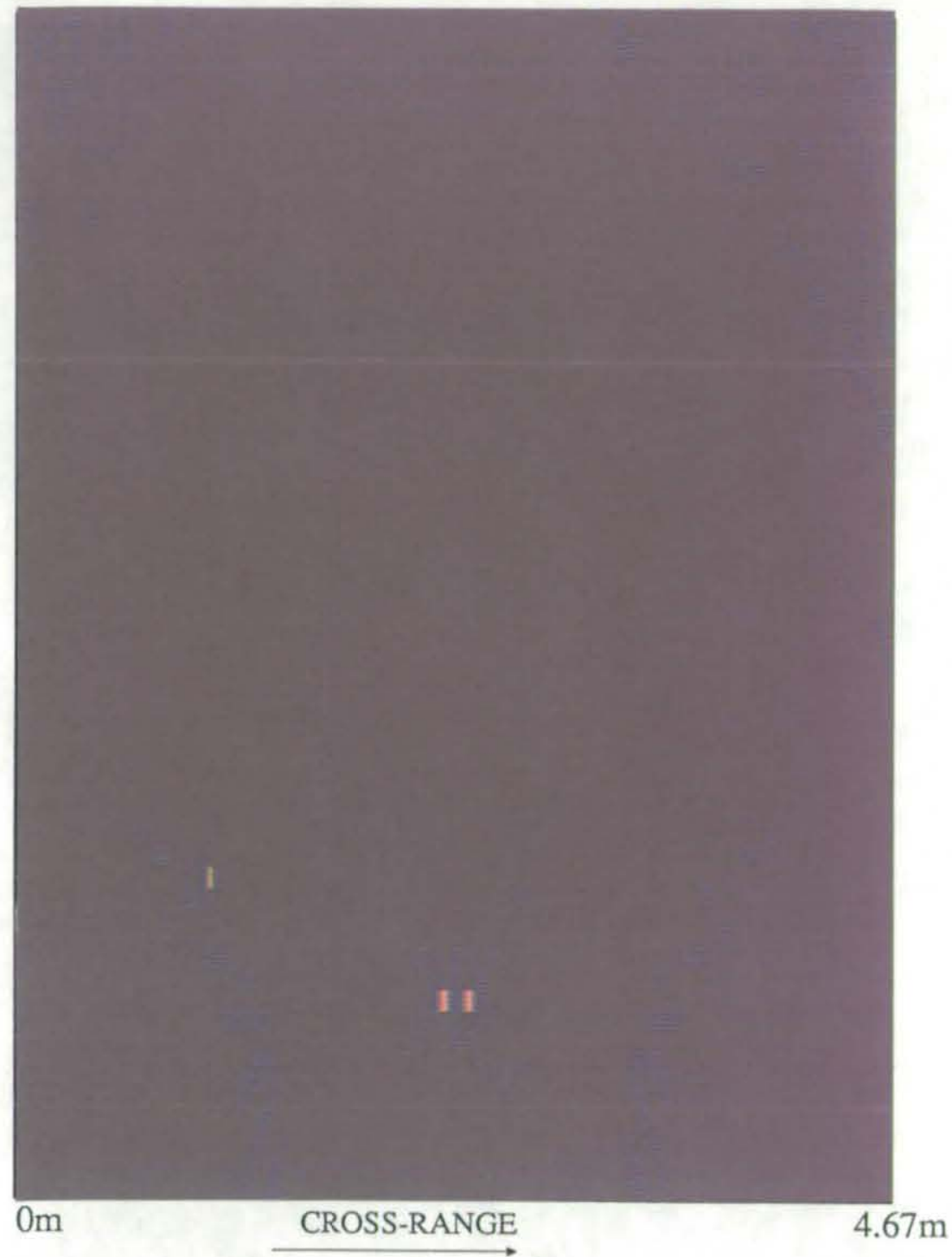

Fig, 7.13 Reconstructed Image of Simulated Data for the Three Nearest Tartgets Shown in Fig, 7.10(a). 


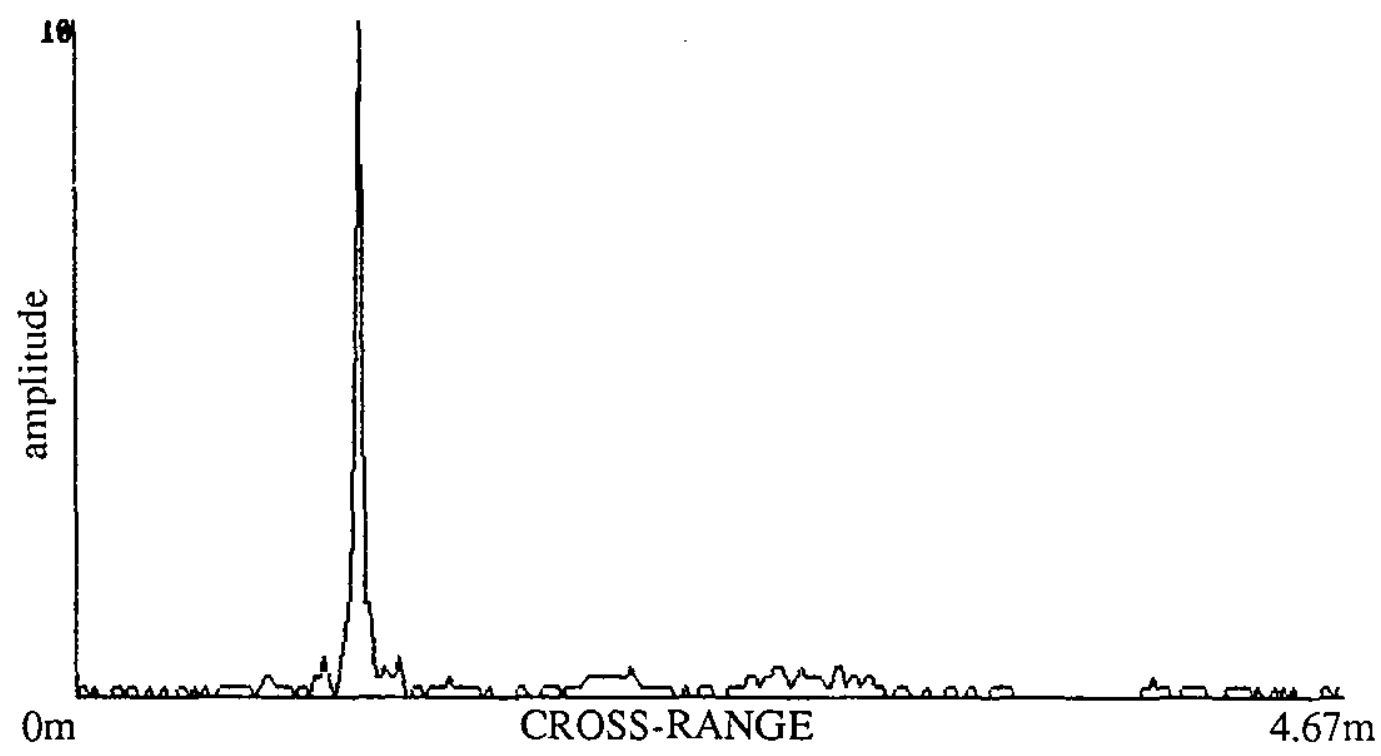

Fig. 7.14 Image Amplitude Variation across Range Cell 31 of Fig, 7.13.

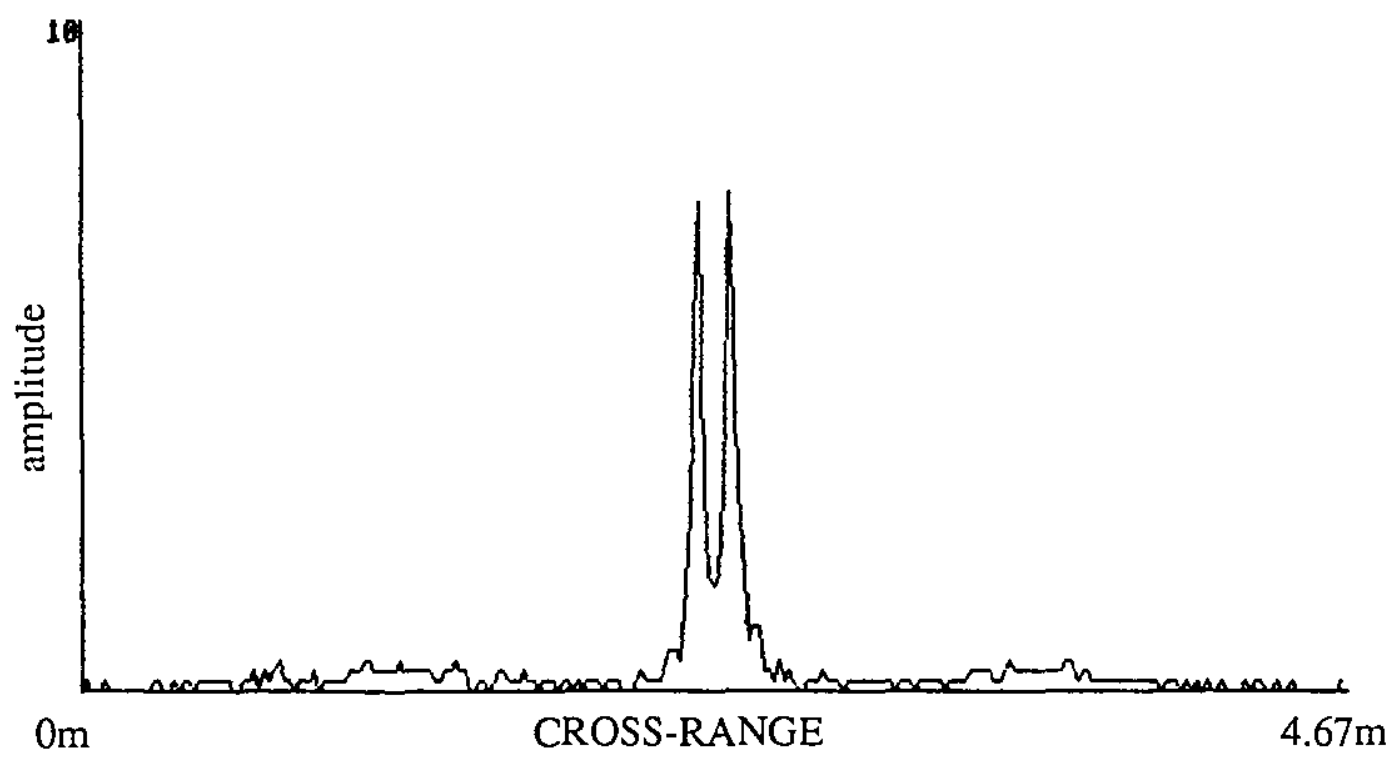

Fig. 7.15 Image Amplitude Variation across Range Cell 20 of Fig, 7.13. 
for the larger target, and Fig. 7.15 for the nearer targets, for comparison with Fig. 7.11 and Fig. 7.12 respectively.

Further examples of target configuration and their reconstructed images are shown in Figs. 7.16 and 7.17 .

\subsection{Experiments at the Reservoir}

The department has an experimental facility at a local reservoir which enabled longer apertures to be synthesized and because of the practical problems of generating a horizontal aperture, the transducers were moved vertically in the water to synthesize a vertical aperture. The water depth at the draw-off tower is about $24 \mathrm{~m}$ but the concrete weights holding the ropes taut and parallel are suspended above the bottom. and so the maximum traverse length is less than $20 \mathrm{~m}$. The images in this section have a cross-range distance of $9.4 \mathrm{~m}$ (unless the image is generated by the non-coherent addition of two or more images) and a maximum range of $18.5 \mathrm{~m}$ when the sampling frequency is $5 \mathrm{kHz}$ and a maximum range of $46 \mathrm{~m}$ when the sampling frequency is 2 $\mathrm{kHz}$.

Fig. 7.18 illustrates the side view of the draw-off tower at the reservoir showing the flat concrete reservoir bottom near the draw-off tower extending to about $11 \mathrm{~m}$ from the tower and then falling of steeply. The reservoir 'drain plug' is a large rectangular concrete structure on the reservoir floor at a range of about $30 \mathrm{~m}$ from the draw-off tower. 


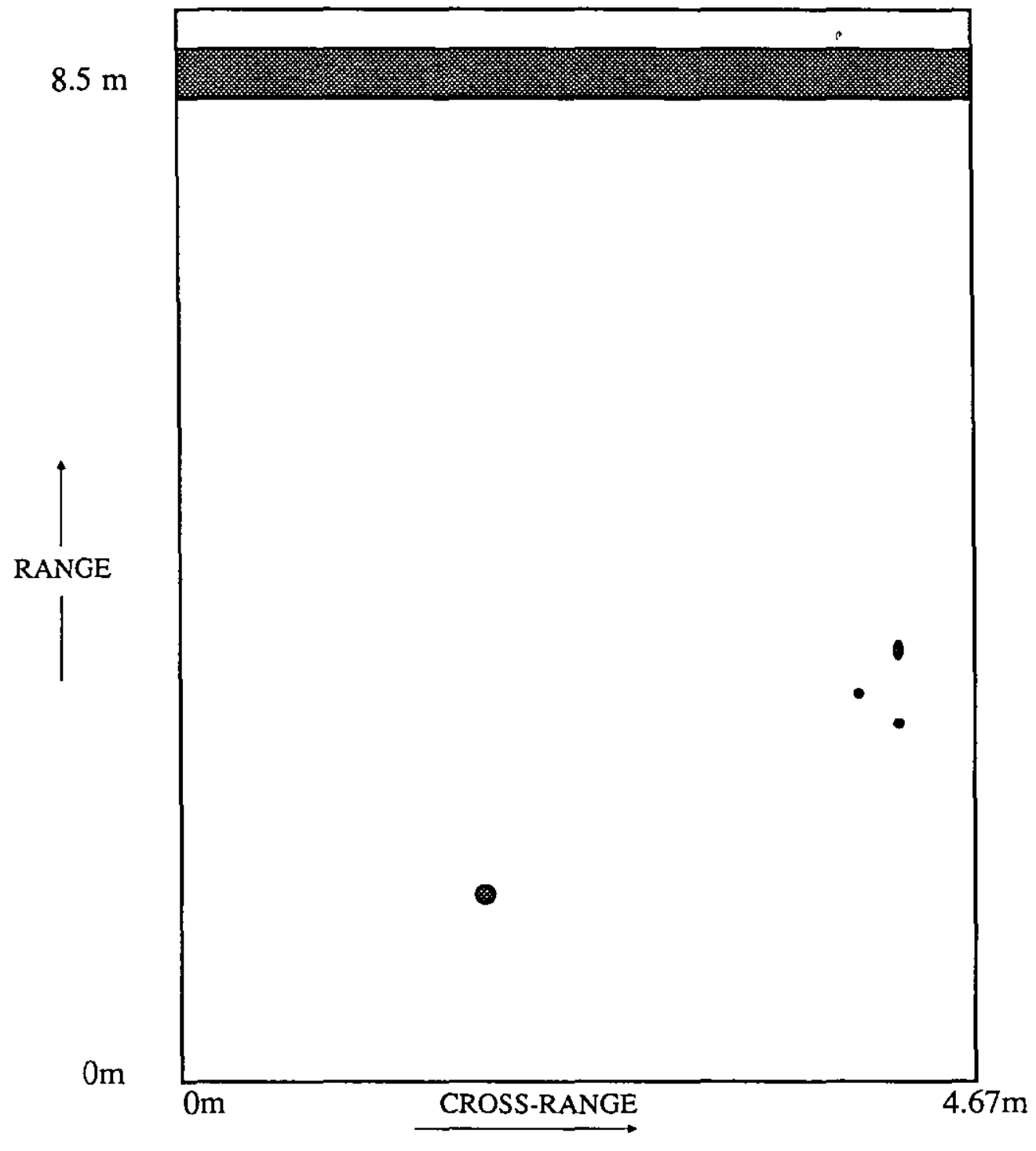

Fig, 7.16(a) Target Configuration 


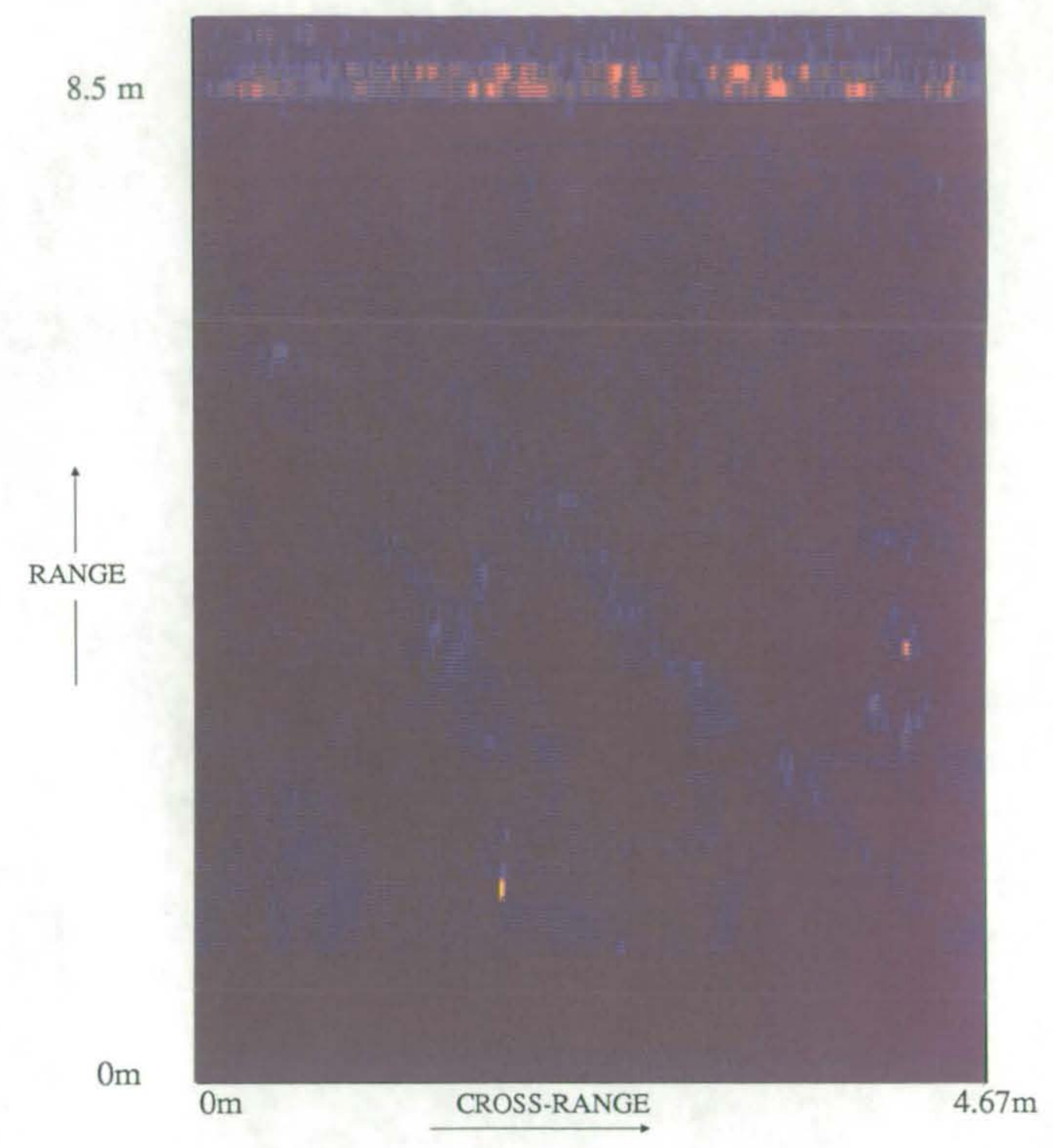

Fig. 7.16(b) Reconstructed Image of the Target Configuration shown in Fig, 7.16(a). 


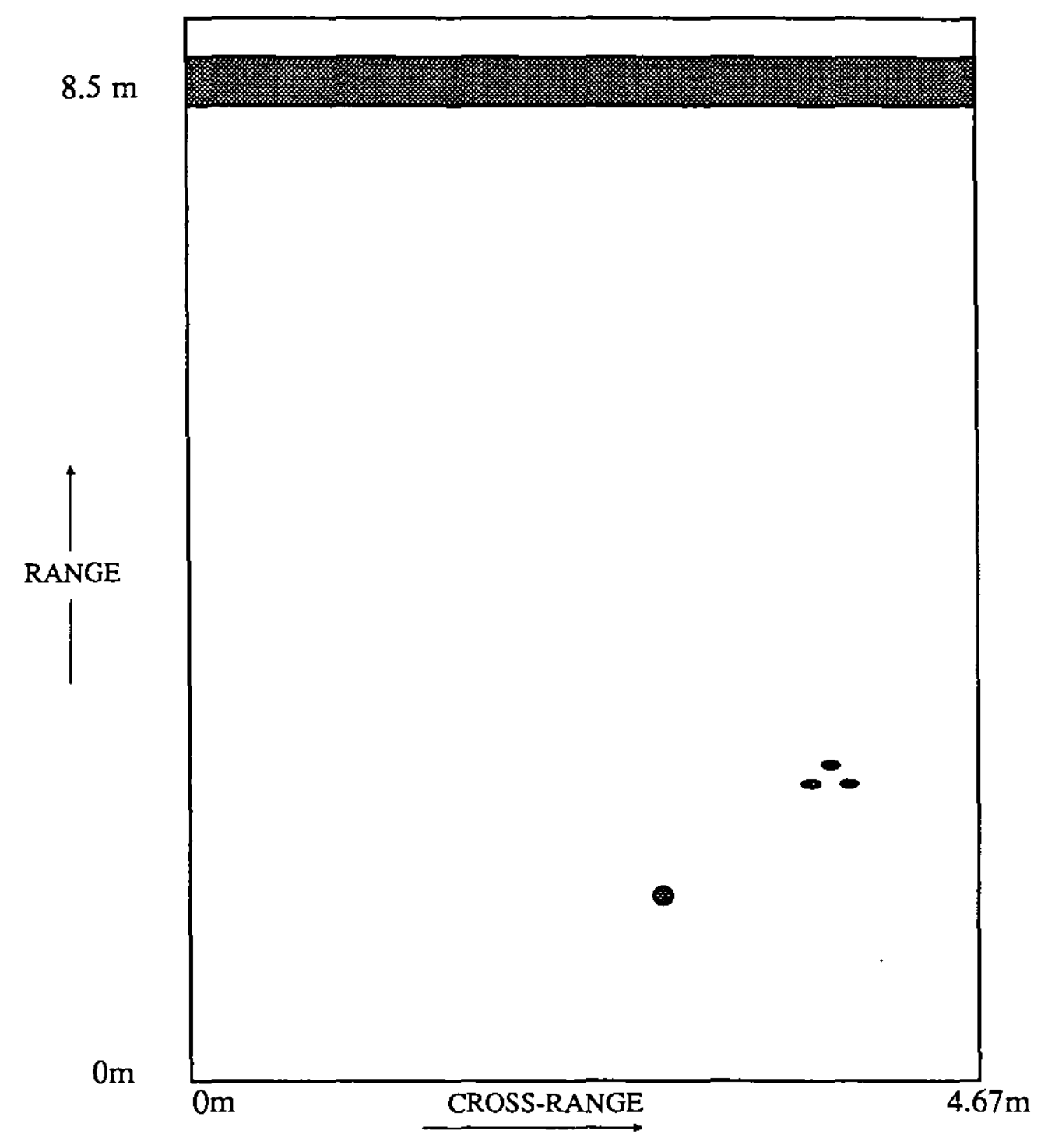

Fig. 7.17(a) Target Configuration 


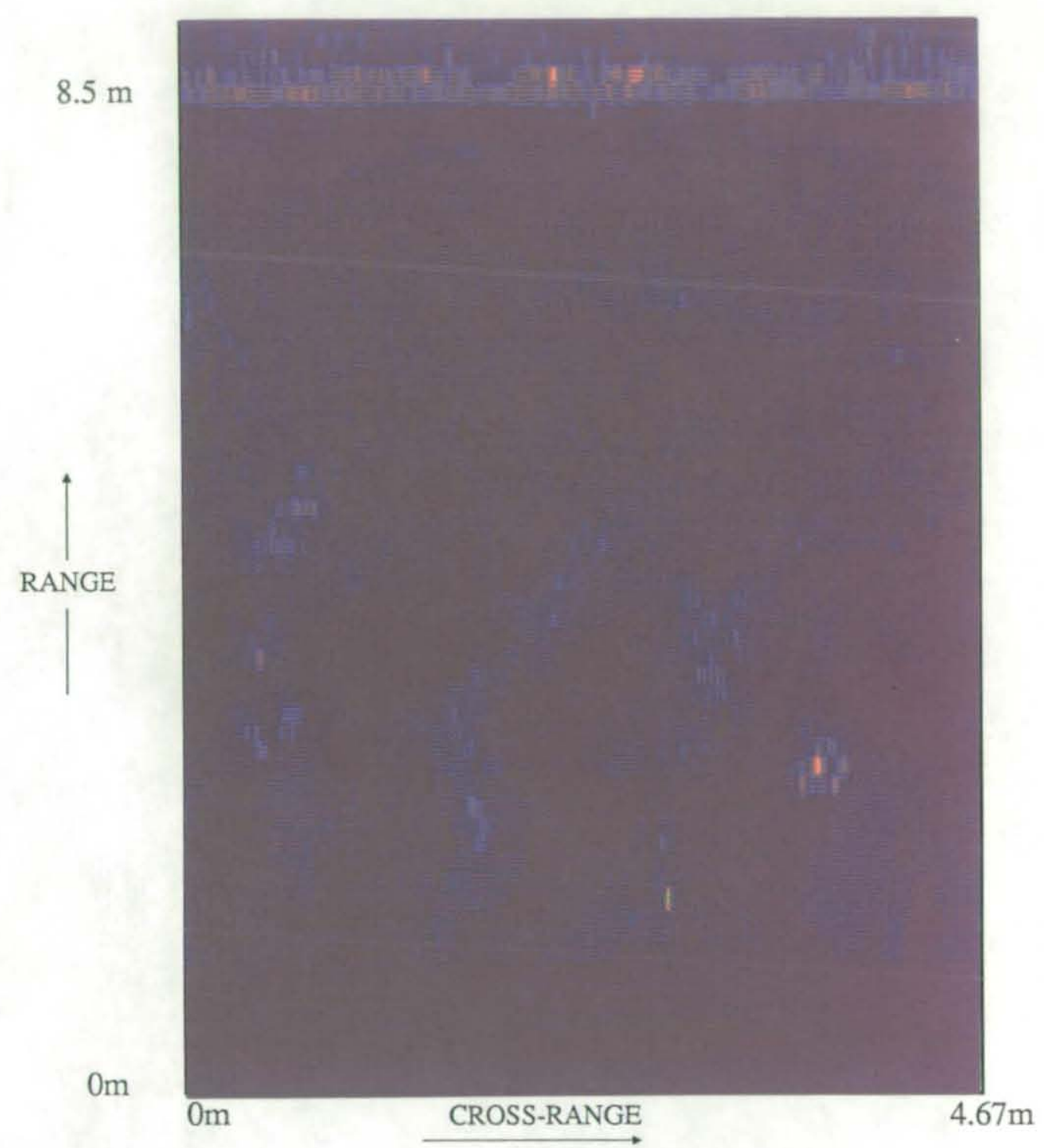

Fig. 7.17(b) Reconstructed Image of the Target Configuration shown in Fig, 7.17(a). 


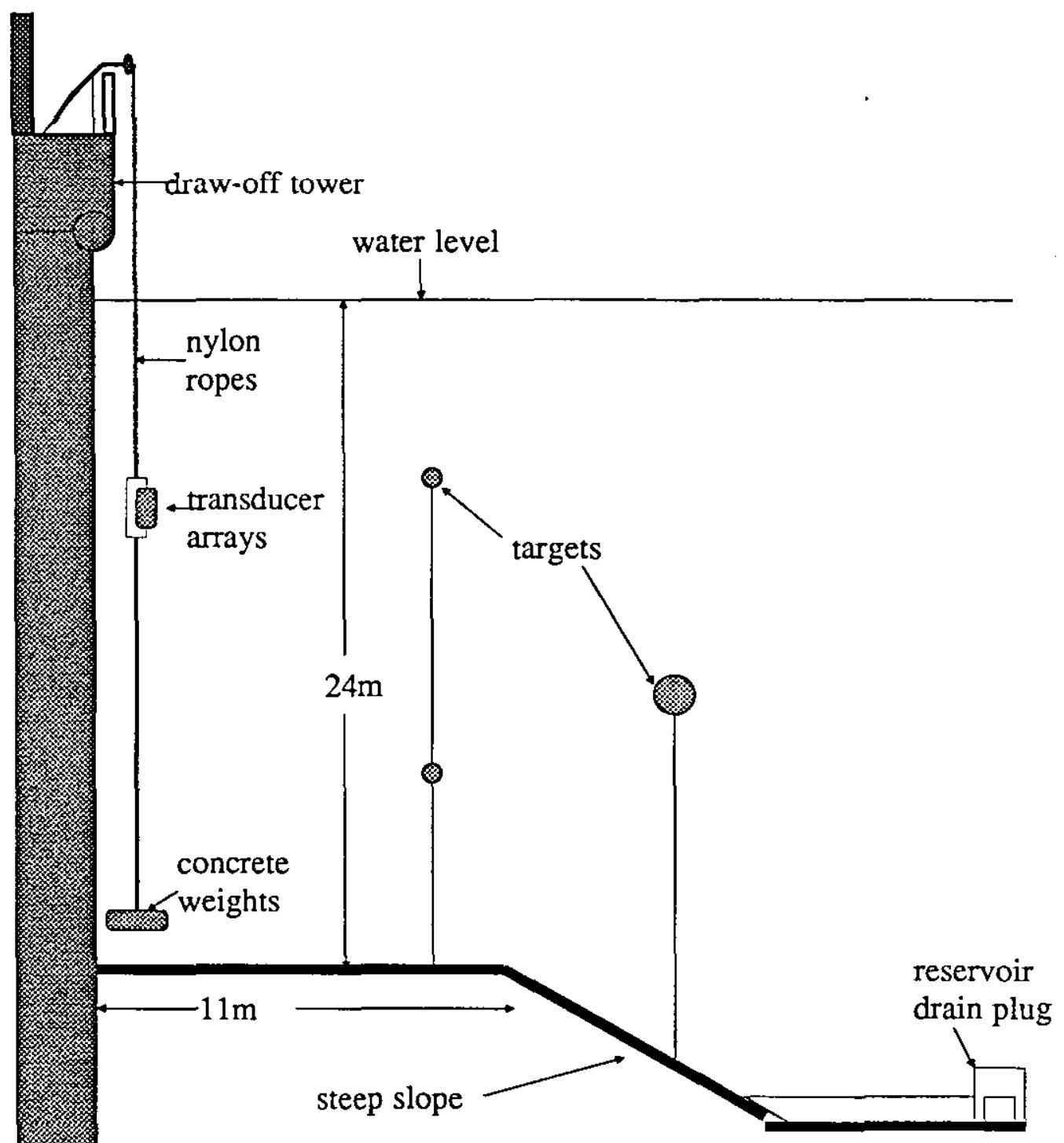

Eig. 7.18 Side View of the Setup at the Reservoir Illustrating the nature of the Bottom Near the Draw-off Tower. 


\subsubsection{Imoges without Motion Error Correction}

The images obtained using the $40 \mathrm{kHz}$ system at the reservoir ore shown in this section. The motion errors are predominantly due to the unknown movement of the transducers caused by wind, waves and underwater currents. The currents can be significant as large quantities of water is pumped out at the draw-off tower.

Images shown in this section have not been corrected for transducer error motion or image processing carried out in the reconstruction process.

Fig. 7.19 to Fig. 7.21 show a variety of reconstructed images with a maximum range of $46 \mathrm{~m}$. Fig. 7.19 shows the reconstruction of a group of targets at a range of $5 \mathrm{~m}$ and a single target at $20 \mathrm{~m}$. Fig. 7.20 shows a target at $15 \mathrm{~m}$ and two targets at $27 \mathrm{~m}$ with a separation of approx. $5 \mathrm{~m}$. Fig. 7.21 shows the same target configuration as Fig. 7.20 but a calibrated hydrophone used for other experimental work was lowered into the water and the co-axial cable used to suspend and drive the hydrophone is clearly visible at a range of $8.5 \mathrm{~m}$.

Fig. 7.22 and Fig. 7.23 illustrates images of a group of targets at various ranges with a maximum displayed range of $18.5 \mathrm{~m}$. Fig. 7.22 is the reconstruction of a single target at a range of $12 \mathrm{~m}$ and $F i g .7 .23$ shows the effects of rain and very windy conditions: the targets have not been successfully reconstructed and spurious targets have been generated.

In all the above images there is the presence of low level noise and the targets appear blurred and smeared and sometimes not reconstructed at all. The strong diagonal 


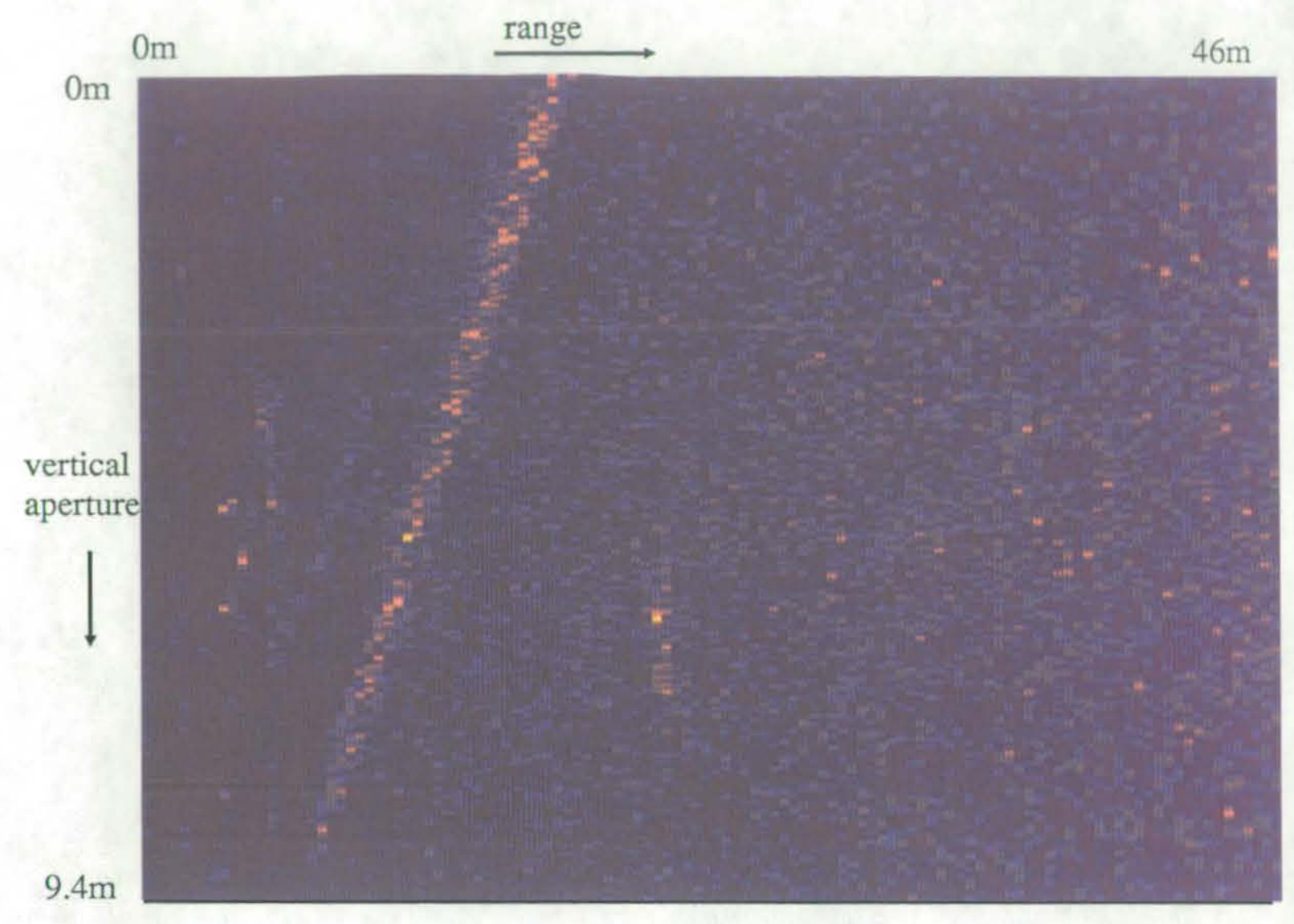

Fig. 7.19 Reconstructed Image of data from the Reservoir without Transducer Error Motion Correction or Image Processing. 


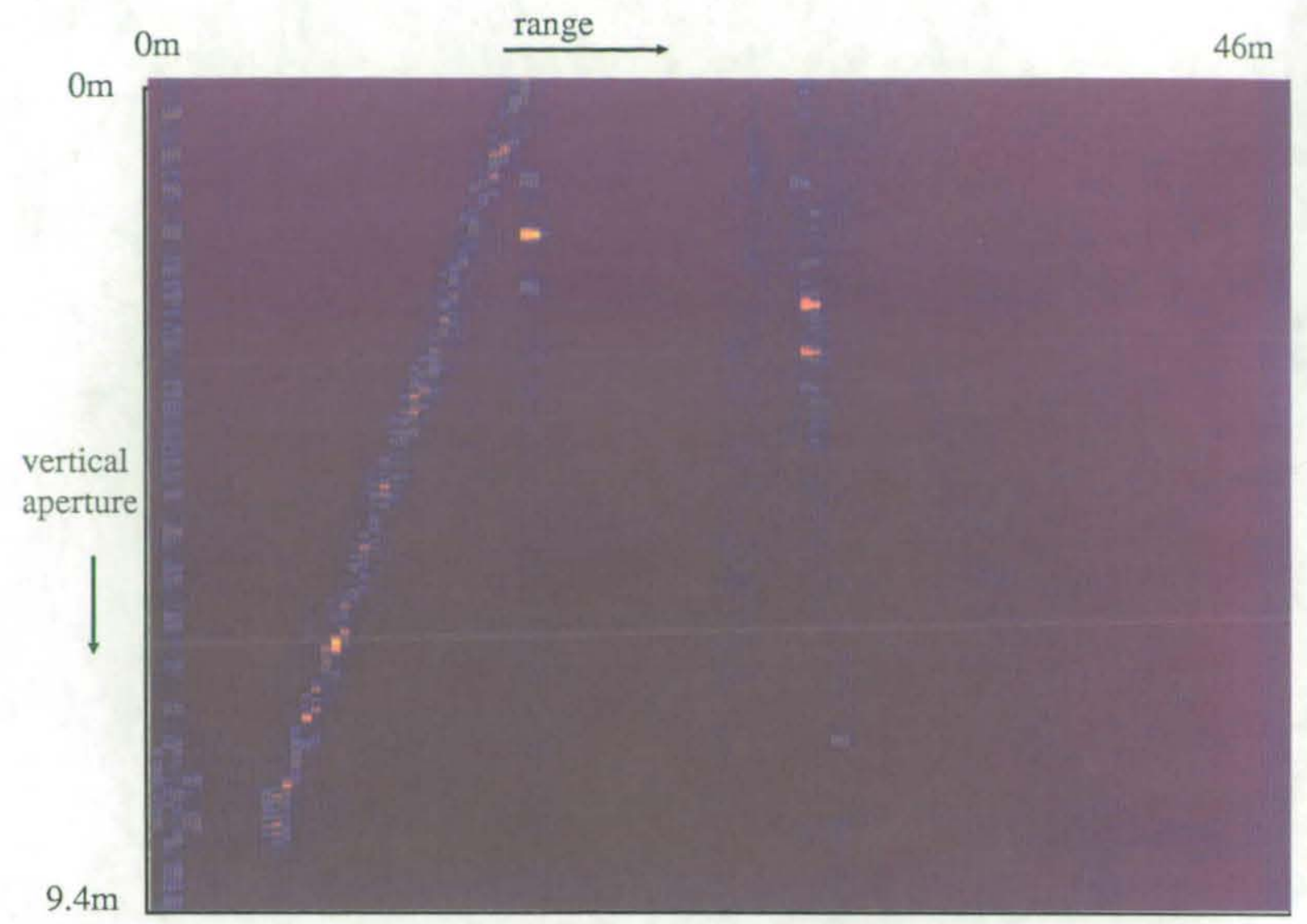

Fig. 7.20 Reconstructed Image of data from the Reservoir without Transducer Error Motion Correction or Image Processing.

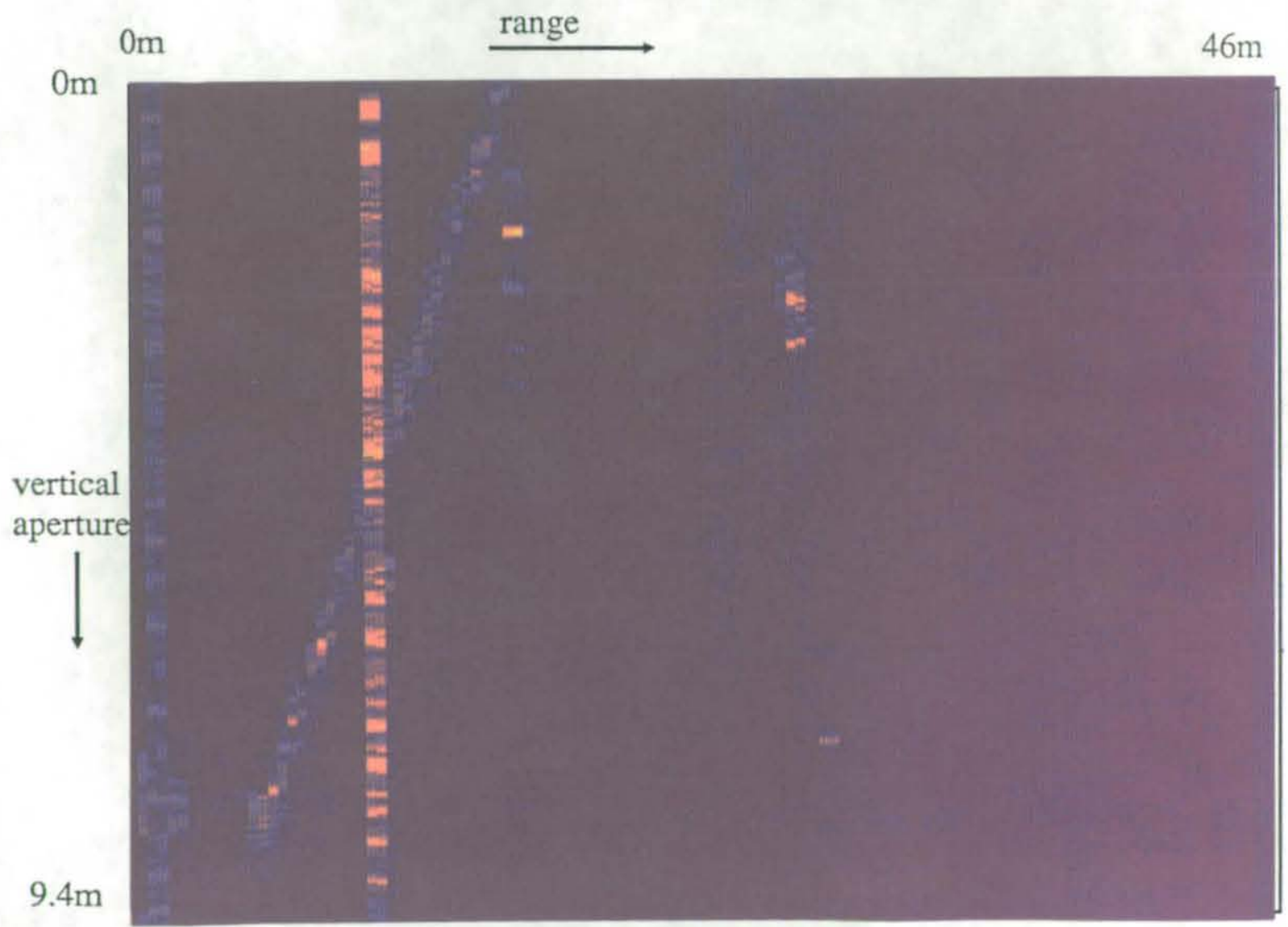

Fig, 7.21 Reconstructed Image of data from the Reservoir without Transducer Error Motion Correction or Image Processing. 


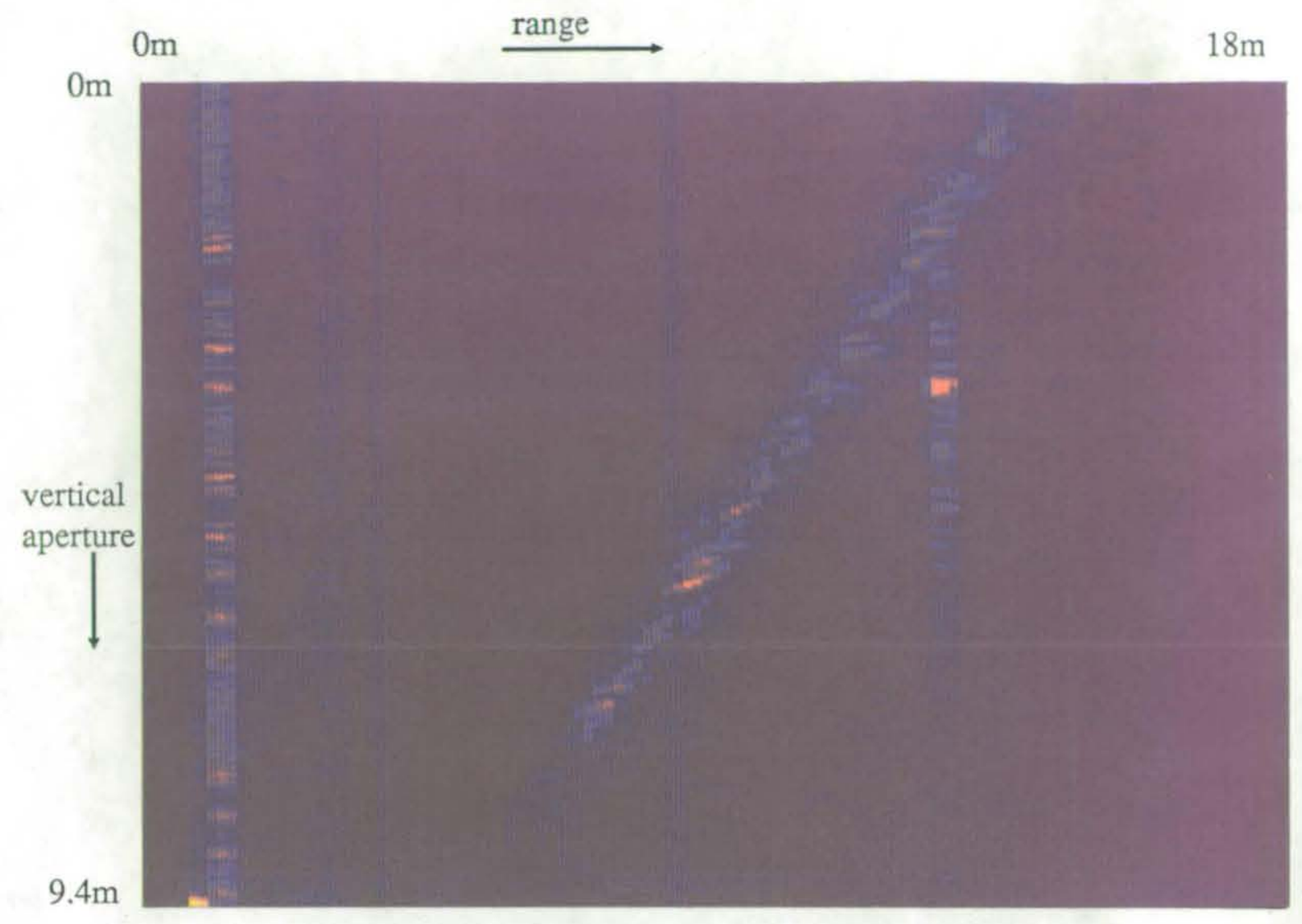

Fig, 7.22 Reconstructed Image of data from the Reservoir without Transducer Error Motion Correction or Image Processing.

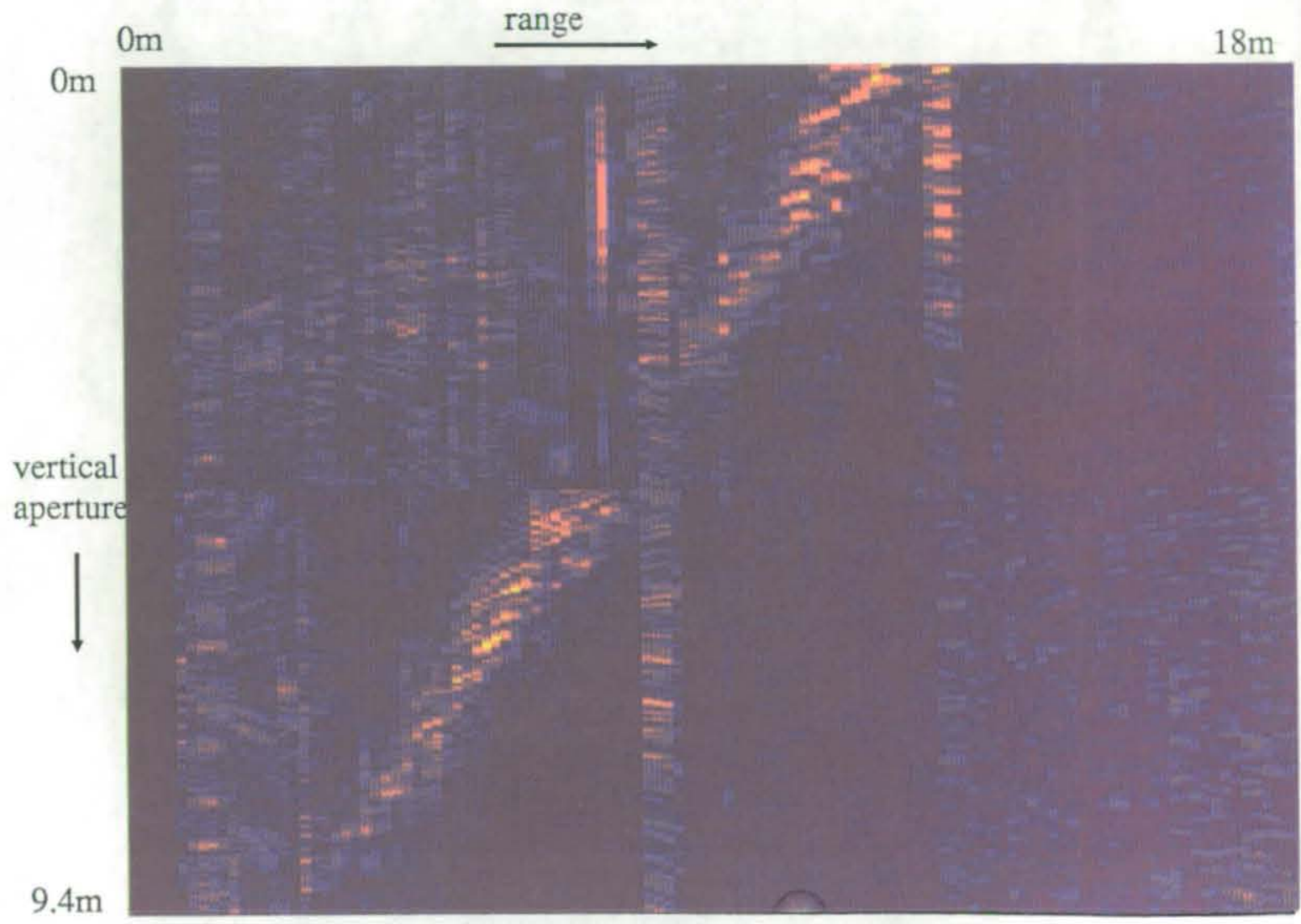

Fig. 7.23 Reconstructed Image of data from the Reservoir without Transducer Error Motion Correction or Image Processing.

$-175-$ 
"target" present in the images is on artefact due to strong reflections from the reservoir bottom near the draw-off tower. If we extrapolate the artefact in the images and using the length of the generated aperture, we can calculate the actual position of the 'target'. Fig. 7.24 shows the extrapolation and we can clearly see that the artefact is due to the reservoir bottom near the draw-off tower. A similar artefact but of much lower amplitude is generated by the water surface.

The effects of a large strong target i.e. the reservoir bottom, on the reconstructed image were simulated and fig. 7.25 illustrates that a similar diagonal artefact is generated. The simulation target configuration also included a target at a range of $12 \mathrm{~m}$ and a poir of smaller targets at a range of $4.6 \mathrm{~m}$ with a separation of $2 \mathrm{~m}$. The artefact due to strong reflections from the reservoir bottom could be eliminated if the exact position of the 'target' was known, but due to fluctuations in the water depth at the reservoir this was impractical. The artefact was used in fact to calculate the position of the aperture with respect to the reservoir bottom by extrapolation as shown in Fig. 7.24 .

This artifact is caused by the strong reflections from the reservoir bottom and surface of the energy in the grating lobes of the synthetic array at $\pm 90^{\circ}$ generated by a sampling distance of $\lambda / 2$ [89]. In an ocean deployed synthetic aperture system this artifact would not be generated as the orray is mounted to provide a wide horizontal beamwidth and moved to generate a long horizontal aperture. The grating lobes generated would be at $0^{\circ}$ and $180^{\circ}$. with respect to the ships heading.

Sorbothane, a material which absorbs acoustic energy 


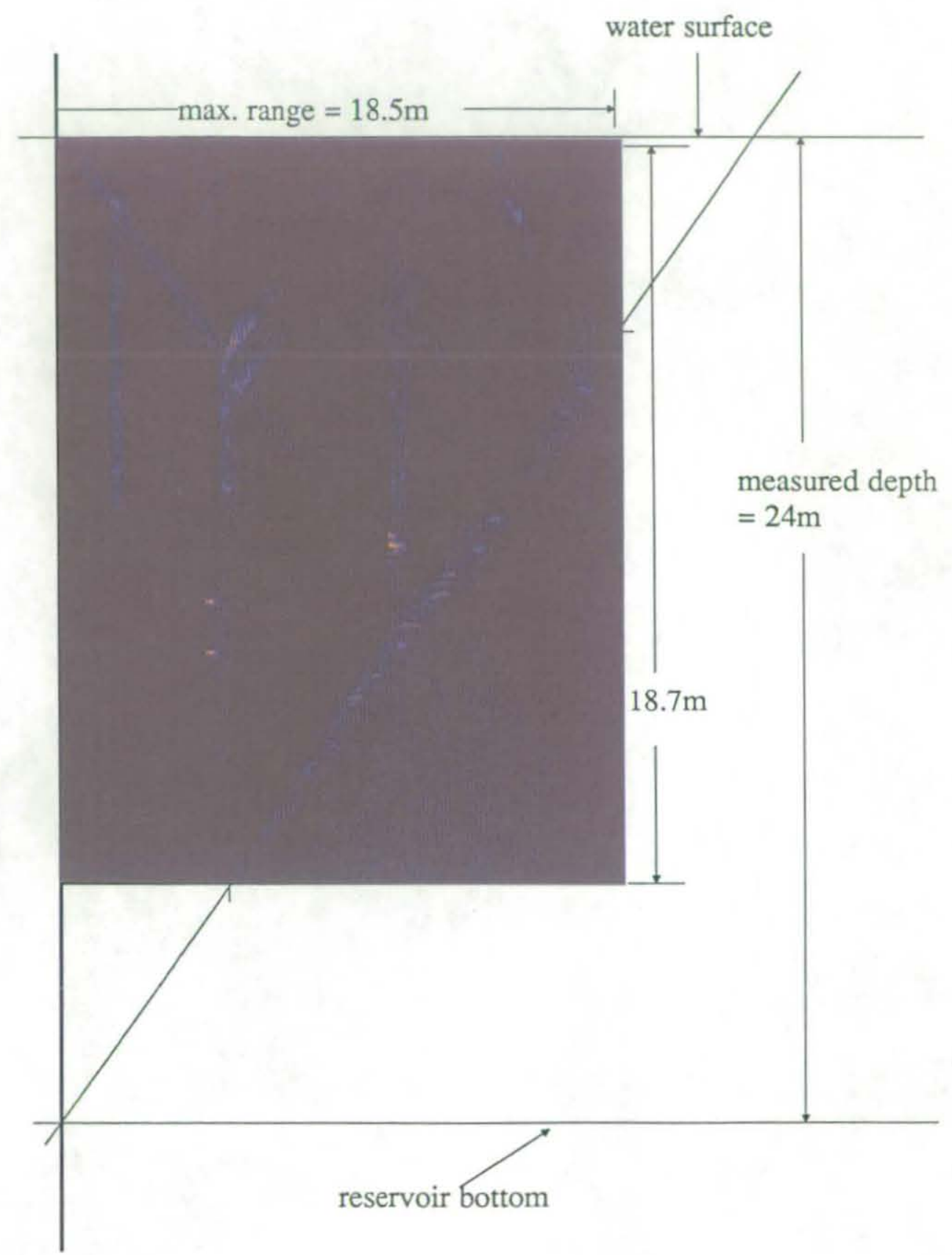

Fig, 7.24 Extrapolation of Reservoir Bottom Artifact to Calculate the Reservoir Depth 


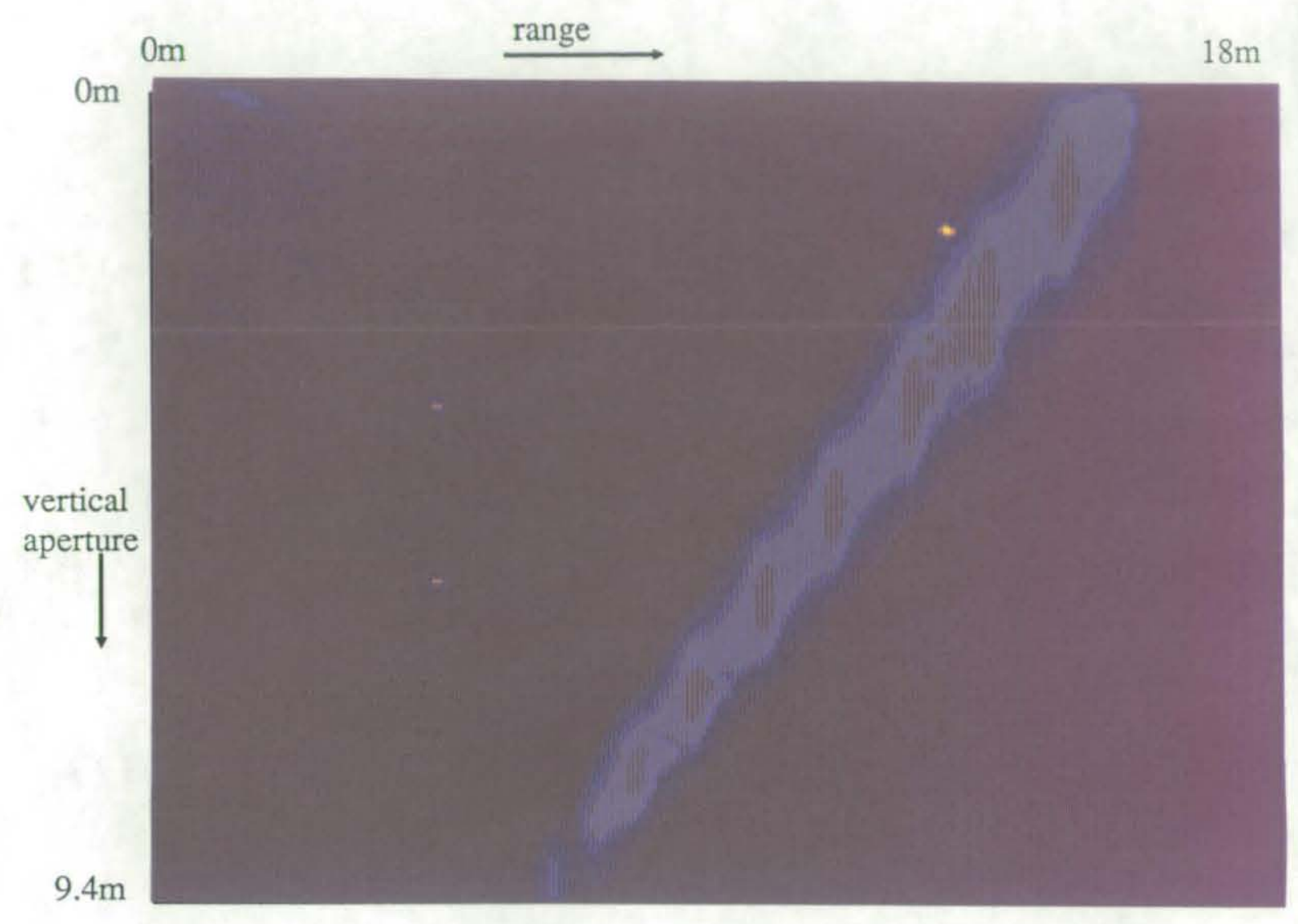

Fig, 7.25 Reconstructed Image of Simulated Data for a Strong Target to the Bottom of the Aperture: An Artifact is Generated 
was used around the sides of the arrays to reduce the amplitude of the artefact generated. Figure 7.22 ilustrates the improvement in the reconstucted image.

7.3.2 Images with Transducer Motion Errors Correction and Image Processing

The images obtained at the reservoir, using the motion error correction technique discussed in chapter 4 and image processing techniques discussed in chapter 5 are illustrated. Two reference sources at ranges of $10 \mathrm{~m}$ and $12 \mathrm{~m}$ with a separation of $6 \mathrm{~m}$ were used.

Fig. 7.26 shows the reconstruction of two $11 \mathrm{~cm}$ diameter air filled spheres at a range of $5 \mathrm{~m}$ with a separation of $1 \mathrm{~m}$. The maximum image range is $18 \mathrm{~m}$.

Fig. 7.27 illustrates a single target at $12 \mathrm{~m}$ range and also one at $25 \mathrm{~m}$ and two targets at a range of $5 \mathrm{~m}$ with a separation of $1 \mathrm{~m}$. The maximum range for this image is $46 \mathrm{~m}$.

The level of background noise is greatly reduced with motion error correction and image processing enhancement and subtractive deconvolution. compared with the earlier images. The image enhancement comprised smoothing convolution masks and histogram modification techniques discussed in section 5.5. The targets are not smeared or blurred and the images appear less 'speckled' and the imoges show a marked improvement in signal to noise ratio and target resolution.

Figs. $7.28,7.29$ and 7.30 are images obtained at the reservoir with various target configurations and corrected 
for transducer error motion correction and image processing included in the reconstruction process and also illustrate the improvement in the resulting images obtained. 


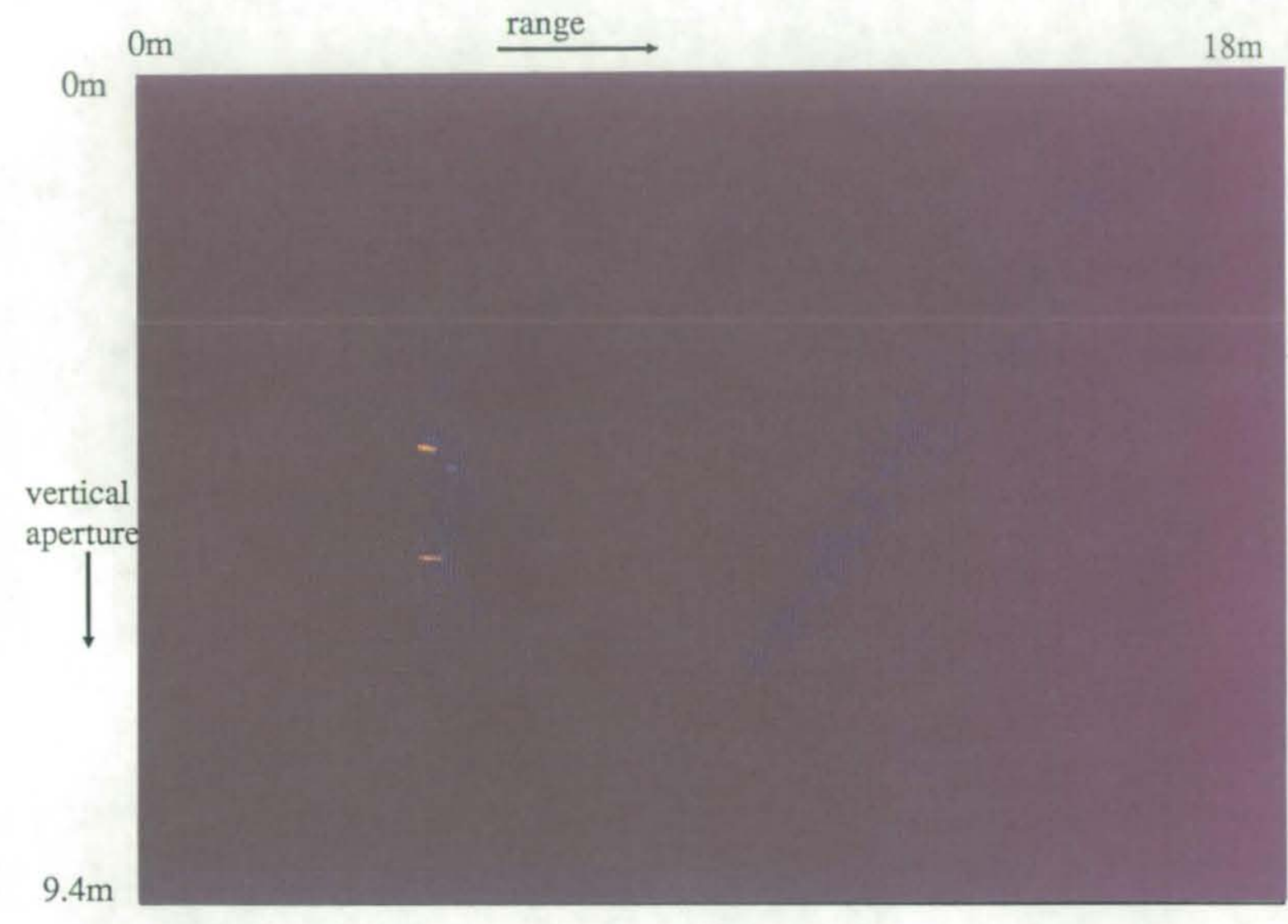

Fig, 7.26 Reconstructed Image of data from the Reservoir with Transducer Error Motion Correction and Image Processing. 


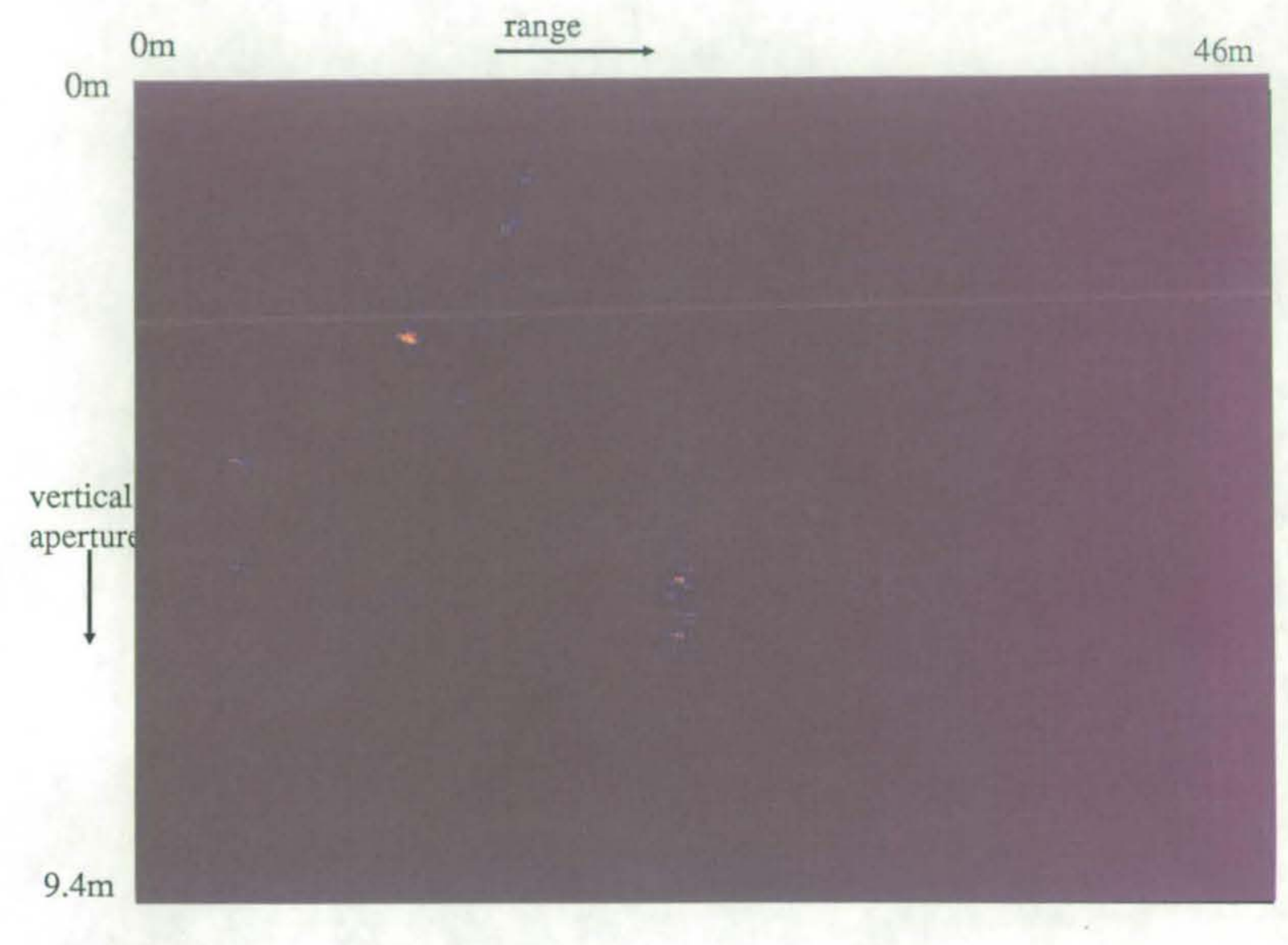

Fig, 7.27 Reconstructed Image of data from the Reservoir with Transducer Error Motion Correction and Image Processing. 
vertical

aperture

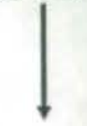

$18.8 \mathrm{~m}$

Fig, 7.28 Reconstructed Images of data from the Reservoir with Transducer Error Motion Correction and Image Processing.

$-183-$ 


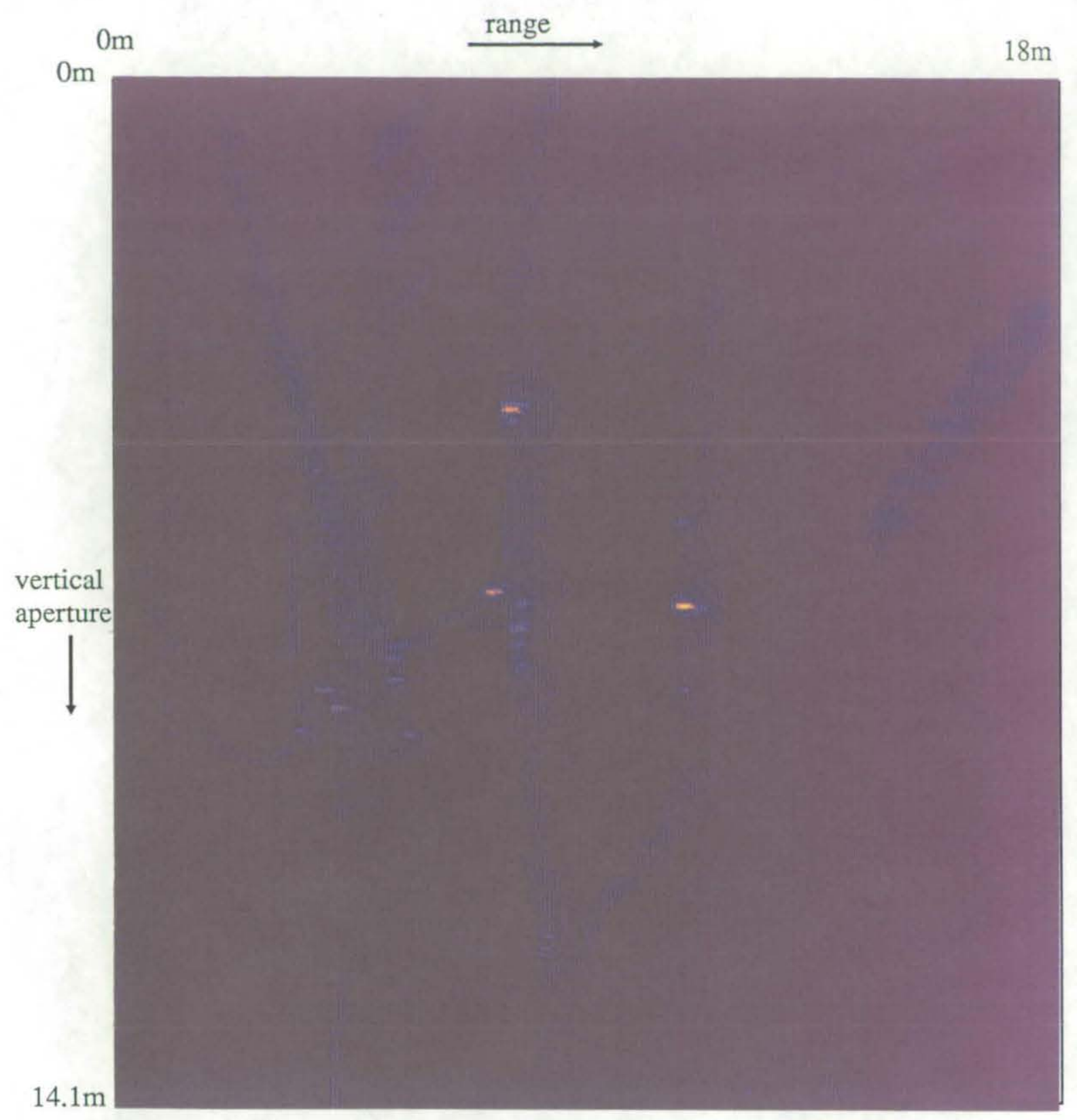

Fig, 7.29 Reconstructed Images of data from the Reservoir with Transducer Error Motion Correction and Image Processing. 


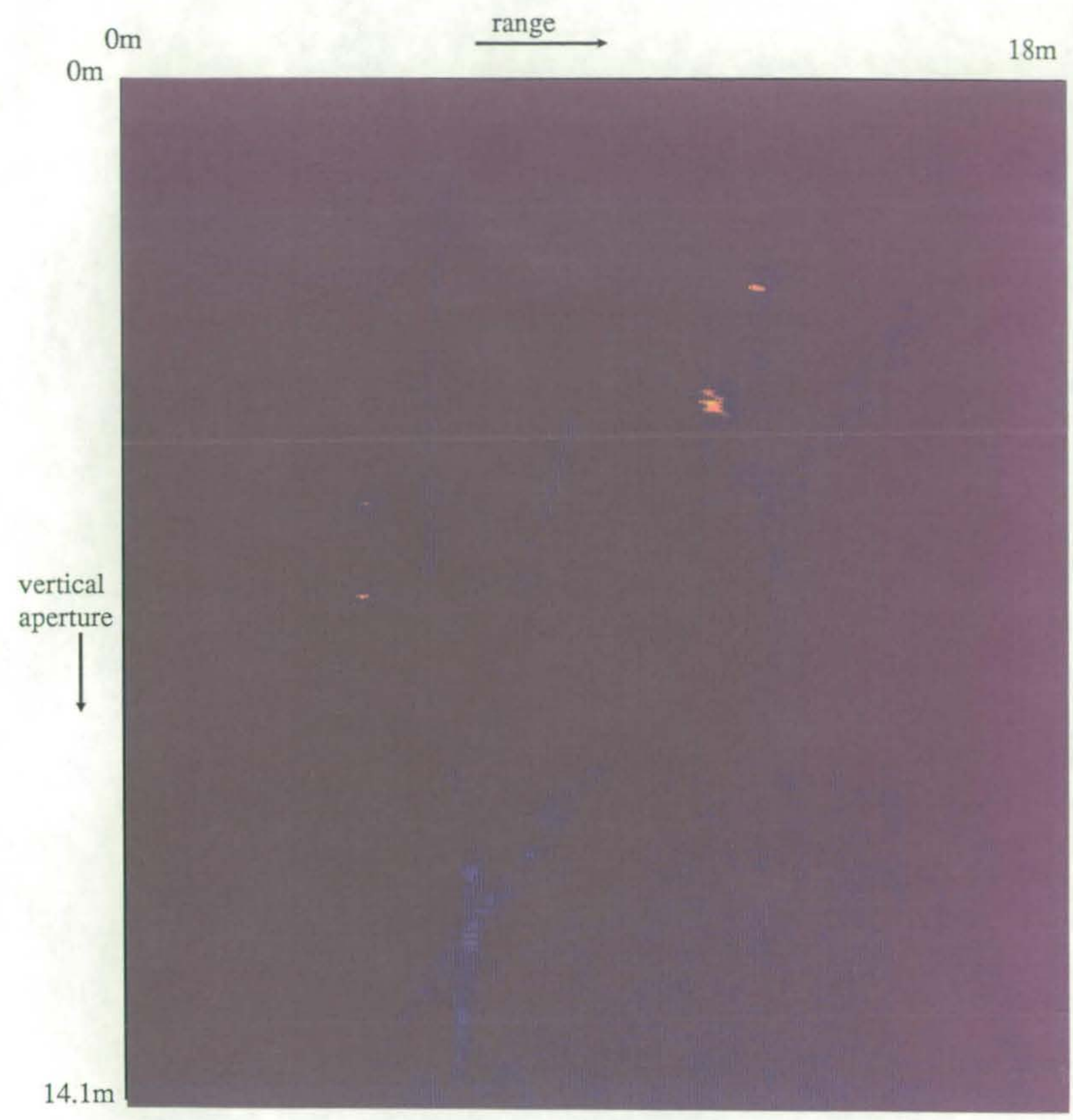

Fig, 7.30 Reconstructed Images of data from the Reservoir with Transducer Error Motion Correction and Image Processing. 


\section{CONCLUSIONS AND}

\section{SUGGESTIONS FOR FUTURE WORK}

The dim of the project was to carry out a study of the possible use of the synthetic aperture technique in underwater acoustics and to consider the parameters of the design of a real-time synthetic aperture imaging system.

Initially a simple computer controlled synthetic aperture sonar system was constructed in a well controlled environment in the department tank. An operating frequency of $150 \mathrm{kHz}$ was used to keep the synthetic aperture length small. The BBC microcomputer was initially chosen as the controlling micro-computer because of its wide range of input/output ports (parallel user port, $1 \mathrm{MHz}$ bus. serial port. fast 'TUBE' inteface) and the large amounts of firmware support. The 8 bit $6502 \mathrm{microprocessor} \mathrm{and} \mathrm{bus}$ structure however restricted its data transfer capabilities in terms of speed and size. For future work the use of one of the newer 16 or 32 bit microprocessors would relieve this problem.

The high resolution images obtained using the synthetic aperture technique relies on the fact that the data collected at all aperture positions must be combined to produce the final image. Optical processing techniques have been used in synthetic aperture systems to obtain the image. However, optical processing does not 
always provide the flexibility, dynamic range or accuracy necessary to produce optimum images. Digital techniques offer the potential for more generalised processing sperations, provide large dynamic range and can correct for system aberrations and other image degrading effects. The data collection procedure included the use of frequency conversion and sampling the quadrature components of the baseband received signal to reduce the sampling rate and data storage requirements. A display based on a framestore was used to observe both the raw data and the reconstructed image. This enabled a quick and visual check on the data as it was collected. The use of separate large banks of digital memory for the data and the image could be used to attain the advantages of non-coherent addition of sub-apertures to reduce the speckle nature of the images obtained. A continuous moving image could also be generated where one sub-aperture is reconstructed whilst the data for the next aperture is stored in a consecutive memory block.

Initially,image reconstruction was carried out using the university Prime mainframe computer. The BBC microcomputer simply acted as a terminal to transfer the stored data to the Prime computer and then to recelve the reconstructed image and display it on the framestore. Data transfer was via the RS423 serial link and when there was a large load on the Prime, the transfer and reconstruction took up to 3 hours. The use of a co-processor comprising a 32 bit microprocessor and a floating point multiplier connected via the fast TUBE interface to the BBC microcomputer. instead of the Prime computer, reduced the reconstruction time to about 20 minutes. The past few years have seen a increase in the availability of very fast digital signal processors (DSP). for example the Texas TMS32000 digital signal processor famlly and the 
introduction of the transputer [90.91.92]. These processors ore ideally suited for the fast parallel digital signal processing required for the successful application of the synthetic aperture technique for real-time imaging. The software generation task for the transputer is made easier by the use of accam. a programming language which has been specially developed for parallel digital signal processing applications.

Once the results from the initial system proved successful and to prepare for the move to a more realistic environment, another system operating at $40 \mathrm{kHz}$ was built. A lower operating frequency was chosen to enable the system to be more resilient to transducer position uncertainty, generate a larger aperture and use the advantages of a lower abosorption coefficient. The $40 \mathrm{kHz}$ system was tested in the department tank before being deployed at a local reservoir, where there was less control over the array motion and water conditions such as turbulence, temperature and velocity profile, and weather conditions of wind and rain.

Traditionally synthetic aperture systems have not been extensively applied to underwater applications due to a variety of factors. The low propagation velocity of sound in water has meant that in order to avoid along track ambiguities the transducer velocity must be small; down to a few metres per hour for long range systems. Higher transducer velocities however may be tolerated by using a multi-frequency system. Turbulence in the medium and transducer position unicertainty have limited the application of synthetic aperture technique in underwater imaging.

( Simulation studies have shown that the effects of 
transducer position uncertainty were target transiation. spurious target generation. target blurring and a reduction in the target to background noise ratio. A method incorporating digital signal processing and image processing techniques was developed and the simulation work proved very successful in reducing the image degradation caused by transducer motion errors. A number of position invariant, point and neighbourhood operations, for image processing applications, have been briefly considered, with the algorithms implemented by discrete convolution in the spatial domain. Sonar images with their low degree of spatial correlation are prone to loss of target detail and blurring with the indiscriminate application of convolution masks and excessive thresholds. A better option is the application of discriminate weighted masks to specific areas in the image to enhance or highlight a particular feature of interest.

Several synthetic aperture sonar systems hove been designed. developed and constructed and their implementation included the use of frequency conversion and sampling of the quadrature components of the baseband received signal to reduce the sampling rate and data storage requirements. A technique for reducing the image degradation due to loss of coherency of the received signal across the generated aperture, caused by transducer motion errors, has been developed and Implemented. Image processing techniques of enhancement and restoration were included in the final reconstruction process to enhance the images and reduce image degradation. 
1. J.H. Blythe.

"A New Type of Pencil Beam Aerial for Radio Astronomy". Monthly Not. Roy. Astron. Soc., Vol. 117. No. 6, pp. 644-651, 1957.

2. C.W. Sherwin, J.P. Ruina, R.D. Rawcliffe.

"Some Early Developments in Synthetic Aperture Radar systems", IRE trans., Vol. MIL-6. No.2. pp. 111-115, April 1962.

3. L.J. Cutrona, W.E. Vivian, E.N. Leith, G. O. Hall.

"A High Resolution Radar Combat-surveillance System". IRE trans., Vol. MIL-5, No.2. pp. 127-131. April 1961.

4. L.J. Cutrona, G. O. Hall.

"A Comparison of Techniques for Achieving Fine Azimuth Resolution". IRE trans. "Vol. MIL-6. No.2. pp.119-121, April 1962.

5. W.M. Brown. L.J. Porcello.

"An Introduction to Synthet ic Aperture Radar". IEEE Spectrum. Vol. 6, pp.52-62. Sept. 1969.

6. L.J. Cutrona.

"Synthetic Aperture Radar", in $\frac{\text { Radar }}{\text { Handbook, M.I. Skolnik. Ed., ch. 23, New }}$
York. McGrow-Hill, 1970.


7. K. Tomiyasu.

"Tutorial Review of Synthetic Aperture Radar with Applications to Imaging of the Ocean Surfoce". IEEE proc., Vol. 66, No. 5 . pp. 563-583. May 1978.

8. L.J. Cutrona, E.N. Leith, L.J. Porcello, W.E. Vivian.

"On the Application of Coherent Optical

Processing Techniques to Synthetic Aperture Rador". IEEE proc. . Vol.54, pp.1026-1032. 1966

9. E.N. Leith, A.L. Ingalis.

"Synthetic Antenna Data Processing by Wavefront Reconstruction". Applied Optics. vol.7. pp. 539-544, 1968.

10. C.B. Burckhardt, P. Grandchamp, H. Hoffmann.

"An Experimental $2 \mathrm{MHz}$ Synthetic Aperture Sonar System Intended for Medical Use". IEEE trans., Vol. SU-21. No.1. Pp.1-6. Jan. 1974.

11. R.J. Urick.

"Principles of Underwater Sound for Engineers". McGraw-Hi / , 1967.

12. D.G. Tucker. B.K. Gazey.

"Applied Underwater Acoustics", Pergamon. London, 1966.

13. C. B. Burckhardt, P. Grandchamp, H. Hoffmann.

"Methods for Increasing the Lateral Resolution of B-Scans". in Acoustic Holography, Vol.5. pp. 391-413, P. Green, Ed. Plenum Press. 1974. 
14. R. Cribbs, S.U. Arnon.

"High Resolution Synthetic Aperture Ultrasound Imaging". Proc. 1st World Fed. Ultrasound Med. Biol. San-Fransisco, 1966.

15. J.C. Kirk.

"A Discussion of Digital Processing in Synthetic Aperture Radar". IEEE trans. Vol. AES-11, No. 3, pp. 326-337. May 1975.

16. J.C. Kirk.

"Digital Synthetic Aperture Radar Technology". Record IEEE 1975 Int. Radar Conf., pp. 482-487. 1975.

17. D.E. Dudgeon.

"Fundamentals of Digital Array Processing". IEEE proc. . Vol. 65. pp. 898-904, June 1977.

18. L.J. Cutrona.

"Comparison of Sonar System Performance Acheivable using Synthetic Aperture Techniques with the Performance Acheivable by more Conventional means". JASA. Vol. 58, No. 2. pp. 336-348, Aug. 1975.

19. R.G. Hughes.

"Sonar Imaging with the Synthetic Aperture Technique", Oceans 77 Conf. record, MTS-IEEE. Vol.1, paper No. 10C, 1977.

20. H.E. Lee.

"Extension of Synthetic Aperture Radar Technique to Undersea Applications". IEEE trans. Vol. $\mathrm{OE}-4$. No. 2, pp. 60-63, April 79. 
21. R. K. Raney.

"Synthetic Aperture Imaging Radar and Moving Targets", IEEE trans. Vol. AES-7. No. 3, pp. 499-505, May 1971.

22. T. Sato, 0. Ikeda.

"Super-resolution Ultrasonic Imaging by Combined Spectral and Aperture Synthesis". JASA, Vol. 62, No. 2, pp. 341-345. Aug. 1977.

23. T. Sato, 0. Ikeda, K. Endo.

"Combined Spectral and Aperture Synthetic Ultrasonic Imaging System". IEEE trans. Vol. SU-28. No. 2, pp. 64-69. March 1981.

24. Y. Pao, A. El-Sherbini, V.C. chen.

"Digital Computer Simulation Study of an Uitrasonic 3-D Imaging System using Frequency Sweep and Synthetic Aperture Technique". IEEE conf. proc. ICASSP. Pp. 815-818. May 1982.

25. T. Sato, 0. Ikeda.

"Super-resolution Imaging System using Waves with a Limited Frequency Bandwidth". JASA, Vol. 65. No. 1. pp. 75-81, Jan. 1979.

26. 0. Ikeda, T. Sato, H. Ohshima.

"Synthetic Aperture Sonar in Turbulent Media". JASA, Vol. 66, No. 1. pp. 209-218, July 1979.

27. 0. Ikeda. T. Sato.

"Further Examinations of Synthetic Aperture Sonar in a Turbulent Media". JASA. Vol. 68. No. 2, pp. 516-522, Aug. 1980. 
REFERENCES

28. J.W. Goodman, W.H. Huntley. D.W. Jackson. M. Lehmann. "Wavefront Reconstruction Imaging Through Random Media", Applied Physics Lett. . Vol.8. No. 12, pp. 311-313, June 1966.

29. W.M. Brown.

"Synthetic Aperture Radar". IEEE trans. Vol. AES-3. PP. 217-229, 1967.

30. L.J. Cutrona, E.N. Leith, C.J. Palermo, L.J. Porcello. "Optical Data Processing and Filtering Systems". IRE trans. Vol. IT-6, p. 386, 1960.

31. J.A. Develet.
"Performance of a Sunthetic Aperture Mapping Radar System". IEEE trans. Vol. ANE-11. No. 3. pp. 173-179, Sept. 1964.

32. M.L. Dick, D.E. Dick, F.D. Mcleod, N.B. Kindig.

"Ultrasonic Synthetic Aperture Imaging". in Acoustical Holography, Vol.7. ed L.W. Kessler. pp. 327-346, Plenum Press. New York, 1977.

33. P.D. Corl, G.S. Kino, C.S. DeSilet, P.M. Grant.

"A Digital Synthetic Focus Imaging System". in Acoustica\} Holography Vol. 8, A.F. Methere!I, pp. 39-53. Plenum Press. New York. 1978.

34. B. Taylor. J.T. Kennair. S.M. Posso, G.P.S. Robinson. "Image Reconstruction from Acoustical Holograms using Microprocessors". Signal Processing Vol. 5. No. 6. pp. 531-540, 1983. 
35. K. Nagai.

"Fourier Domain Reconstruction of Synthetic Focus Acoustic Imaging System". IEEE proc.. Vol. 72, pp. 748-749, June 1984.

36. T. Yomamoto, Y. Aoki.

"Holographic B-Scan Imaging using Wide Band Chirped Ultrasound". IEEE trans. Vol. SU-31. No. 4, pp. 362-366, July 1984.

37. T. Sato, 0. Ikeda.

"Sequential Synthetic Aperture Sonar System A Prototype of a Synthetic Aperture Sonar System". IEEE trans., Vol. SU-24, No. 4, pp. 253-259. July 1977.

38. J.W. Goodman.

"Introduction to Fourier Optics". p.47. McGraw-Hill. New York. 1968.

39. V. Albers.

"Underwater Acoustics Handbook - II". University Press, 1965.

40. C.5. Clay, H. Medwin.

"Acoustical Dceanography: Principles and Applications". Wiley. New York. 1977.

41. D.G. Checketts, B.V. Smith.

"Analysis of the Effects of Platform Motion Errors upon Synthetic Aperture Sonar". Institute of Acoustics proc.. Vol.8. No. 3. pp. 135-143, 1986. 
REFERENCES

42. R. A. Jones, K. Preston.

"Digital Simulation and Processing Applied to Coherent Imaging Systems". IEEE trans., Vol. CAS-22. No. 3, pp. 293-303. March 1975.

43. A. Papoulis.

"The Fourler Integral and its Applications", pp. 130-131. McGrow-Hill. New York, 1962.

44. R.N. Bracewell.

"The Fourier Transformation and its Applications", pp. 267-272, McGraw-Hil1. New York, 1978.

45. J.H. Mimms, L.J. Farrell.

"Synthetic Aperture Imaging with Maneuvers". IEEE trans., Vol. AES-8, No.4, Pp. 410-418. July 1972.

46. L.J. Farrell, J.H. Mimms, A. Sorrell.

"Effects of Phase Errors in Maneuvering Synthetic Aperture Radar". IEEE trans., Vol. AES-9, pp. 758-776. Sept. 1973.

47. W.M. Brown. C.J. Palermo.

"Effects of Phase Errors on Resolution". IEEE trans., Vol. MIL-9. pp.4-9. Jan. 1965.

48. J.A. Develet.

"The Influence of Random Phase Errors on the Angular Resolution of Synthetic Aperture Rodar Systems". IEEE trans.. Vol. ANE-11. No. 1, pp. 58-65, March 1964. 
49. J.C. Kirk.

"Motion Compensation for Synthetic Aperture Radar". IEEE trons. Vol. AES-11. pp. 338-348. May 1975.

50. L.J. Porcello.

"Turbulence-induced Phase Errors in Synthetic Aperture Radar Systems". IEEE trans. "Vol. AES-6, pp. 636-644, Sept. 1970.

51. E. Pusone, L. Lloyd.

"Synthetic Aperture Sonar: An Analysis of Beamforming and System Design". IEEE, ICASSP, Vol.2, p. 33.12.1, 1984.

52. A.W. Rihaczek.

"Radar resolution of Moving Torgets", IEEE trans.. Vol. IT-13. pp. 51-56, Jan. 1967.

53. E.J. Kelly, R.P. Wishner.

"Matched-filter Theory for High Velocity Accelerating Targets" IEEE trans. Vol. MIL-9, pp. 56-59, Jan. 1965.

54. H. Ermert, R. Karg.

"Multifrequency Acoustical Holography". IEEE trans. Vol. Su-26. No.4. pp. 279-286. July 1979.

55. M. Ueda, T. Sato, O. Ikeda.

"Ultrasonic Holography Free from Phase Turbulence". JASA, Vol. 55, pp. 1218-1224, 1974. 
REFERENCES

56. R.E. Williams.

"Creating an Acoustic Synthetic Aperture in the Ocean". JASA, Vol. 60, pp. 60-73, July 1976.

57. H.L. McCord.

"The Equivalence Amongst Three Approaches to Deriving Synthetic Array Patterns and Analyzing Processing Techniques", IRE trans.. Vol. MIL-6, pp. 116-119. April 1962.

58. R.C. Heimiller.

"Theory and Evaluation of Gain Patterns of Synthetic Arrays". IRE trans., Vol. MIL-6, pp. 122-129, April 1962.

59. C. A. Greene, R. T. Moller.

"The Effects of Normally Distributed Random Phase Errors on Synthetic Array Gain Patterns", IRE trans.. Vol. MIL-6, pp.130-139. April 1962 .

60. J. Coll.

"The BBC Microcomputer: User Guide", B.B.C. . 1982 .

61. A.C. Bray, A.C. Dickens, M. A. Holmes.

"B.B.C. Advanced User Gulde". Cambridge Press, 1983 .

62. A.D. Derrick.

"A Hardware Guide for the B.B.C.

Microcomputer". Wise OWl, 1983. 
REFERENCES

63. N. Cryer.

"Graphics on the B.B.C. Microcomputer". Prentice-Hall, 1983.

64. B. Smith.

"B.B.C. Micro - Assembly Language". Shiva, 1983.

65. B. Smith.

"Interfacing Projects for the B.B.C.

Microcomputer". Addison-Wesley. 1984.

66. ACORN.

"32016 Second Processor User Guide and Reference Manual". Acorn, 1985.

67. R. O. Harger.

"Synthetic Aperture Radar System: Theory and Design". Academic Press. New York, 1970.

68. C. Elachi, D.D. Evans.

"The Effects of Random Phase Changes on the Formation of S.A.R. Imagery". IEEE Journal. Vol. OE-2, No.1. pP. 149-153, Jan. 1977.

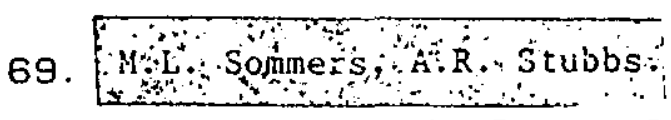

"Side-Scan Sonar". IEE proc. part F. Vol.

131. No. 3. pp. 243-256. June 1984.

70. W.D. Chesterman, P.R. Clynick, A.H. Stride.

"An Acoustic Aid to Sea Bed Survey". Ac. ustica, Vol. 8, pp. 285-290. 1958. 
REFERENCES

71. A.H. Stride.

"Narrow Beam Echo-ranger for Fishery and Geological Investigations". Journ. Mar. Biol. Assoc. U.K., Vol. 38, pp. 313-318, 1959.

72. F.G. Smith.

"Radio Astronomy", p. 228, Penguin, 1960.

73. Severn Trent Water Authority - South Derbyshire reservoir at Foremark.

74. J.A. Richards.

"Remote Sensing Digital Image Analysis: An Introduction". p.109, Springer-Verlag. 1986.

75. K.D. Baker.

"Basic Image Processing Concepts" in Digital Signal Processing ed. N.B. Jones. IEE Control Eng. Series 22, pp. 287-318, Peregrinus, 1983.

76. R.H.T. Bates, M. J. McDonnel I.

"Image Restoration and Reconstruction". Oxford Eng. Science Series 16, P.80. Clarendon, 1986.

77. R.H.T. Bates, et al.

"Practical Image Processing". Physics in Technology. Vol. 9. pp.101-107. 1978.

78. J.A. HÖgbom.

"Aperture Synthesis with a Non-regular Distribution of Interferometer Baselines". Astronomy \& Astrophysics Supplement Series. Vol. 15, p.417. 1974. 
REFERENCES

79. R.E. Graham.

"Snow-Removal: A Noise Stripping Process for

Picture Signals", IRE trans. Vol. IT-8. No.1. Pp. 129-144, 1962.

80. J.L. Hilburn, D.E. Johnson.

"Manual of Active Filter Design", p. 51.

McGrow-Hill. 1984.

81. G. Kozak.

"Side Scan Sonar: A Tool for the Diving Industry", 10th Int. Diving Symp. . New Or leans USA, Feb. 1980.

82. A. Rosenfeld.

"Picture Processing by Computer".

Academic Press, New York, 1969.

83. R.C. Gonzalez, P. Wintz.

"Digital Image Processing".

Addison-Wesley, 1977.

84. W.K. Pratt.

"Digital Image Processing".

wi ley, New York, 1978.

85. L.R. Rabiner. C.M. Rader (ed).

"Digital Signal Processing".

IEEE Press. New York. 1972.

86. M.M. Sondhi.

"Image Restoration: The removal of Spatially Invariant Degradations". Proc. IEEE, Vol. 60. No. 7. pp. 842 - 853, July 1972. 
87. H.C. Andrews, B. R. Hunt.

"Digital Image Restoration".

Prentice-Hall, 1977.

88. A.V. Oppenheim, R.W. Schafer. T.G. Stockham.

"Non Linear Filtering of Multiplied and Convolved Signals", proc. IEEE, Vol. 56, pp. 1264 - 1291. Aug. 1968.

89. B.D. Steinberg.

"Principles of Aperture and Array System Design“, p. 74, Wiley, 1976.

90. A.W. Rudge, K. Milne, A.D. Olver. P. Knight (eds).

"The Handbook of Antenna Design", p. 41. IEE

Electromagnetic Series. Peregrinus, 1986.

91. D.I. Jones, P.J. Flemming.

"Parallel Processing for Real-Time Control System". IEE Colloquium, Parallel Processing:

A New Direction for Control?. Feb. 87.

92. D. Rolfe.

"Parallel Processing using the Transputer". Computing Techniques. Vol.2. pp. 15 - 19. March 1987.

93. D. Taylor.

"Parallel Processing in Occam". IERE 2nd Colloquium. Parallel Processing. Parallel Thinking. Sept. 86. 


\section{FRAMESTORE}

\section{A1. 1 Introduction}

The R16.4.2/2BH monochrome framestore developed by the British Telecom Research Centre is used to provide an inexpensive source of data storage and a display for the final reconstructed image. Individual pixels are accessed by storing their $x$ and $y$ co-ordinates in address latches and then executing a read or write operation.

A brief description of the operation of the framestore will be given, a more detailed explanation can be obtained from R16.4.2/2BH User Manual by British Telecom research centre.

The monochrome framestore can display 64 levels ( 6 bits) of grey shading. from black (00) to white (63) and can be configured in 3 resolution modes:
(i) 4 stores of 256 by 256 pixels.
(ii) 2 stores of 512 by 256 pixels.
(iii) 1 store of 512 by 512 pixels.

\section{A1. 2 BBC Interfoce}

The framestore is interfaced to the BBC microcomputer via the $1 M H z$ Bus. Fig. Al.1 shows the ribbon cable connections. The framestore requires 10 address I ines, 8 data lines, a read/write line, a clock 1 ine and a 
$1 \mathrm{MHz}$ BUS

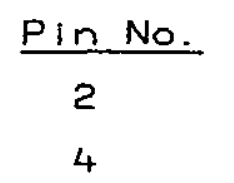

18

19

20

21

22

23

24

25

27

28

29

30

31

32

33

34

10

$1,3,5,7,9$

11.13

15,17

6

8

12

14

16
Signal

$R / n W$

IMHZE

DO

01

D2

D3

04

D5

D6

D7

AO

A1

A2

A3

A4

A5

A6

A7

NPGFC

Ov

ov

Ov

NNMI

NIRQ

NPGFD

NRST

ANALOG IN

\section{FRAMESTORE}

Signal

$\mathrm{R} / \mathrm{nW}$

Pin No.

25

$E / \Phi 2$

24

DO

1

D1

2

D2

3

D3

04

4

5

D5

6

D6

07

$A 0$

11

A7

12

A8

13

A9

14

A10

15

A11

16

A12

17

A13

18

VMA

23

OV

9.10

Dv

19,20

Ov

21,22

$+5 v$

26

Fig. A1. 1 Ribbon Cable Connections between BBC and Framestore 
valid memory address ( VMA) line. The $1 \mathrm{MHz}$ bus provides these in the form of the data bus, $R / W$ control $l$ ine, a $1 \mathrm{MHzE}$ clock line and a NPGFC signal. The framestore is located at address \&FCDO on the $1 \mathrm{MHz}$ bus.

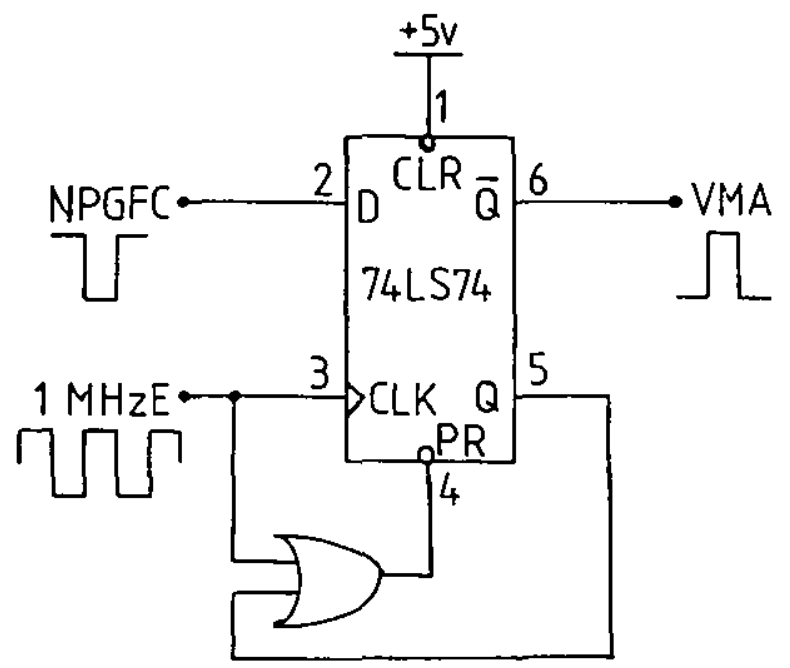

Fig. A1.2 VMA Clean-up Circuit.

A clean up circuit, which is shown in Fig. A1.2, is used to remove any glitches which may be present on the NPGFC control signal line. The operation of the circuit is as follows: before the VMA line can go high, a NPGFC with $1 M H z E$ low must occur. The NPGFC is then latched into the d-type flip-flop on the next rising edge of the $1 \mathrm{MHzE}$ clock. The VMA line will go high a time lag of 40 ns after the $1 \mathrm{MHzE}$ line has gone high and will remain high until 40ns after the 1MHzE line has gone low again. Any transitions in the NPGFC line that occur at any other time will not be passed through the flip-flop.

\section{A1.3 Fast/Slow Access}

There are two rates at which the microcomputer can 
access the fromestore. In the slow mode, the read/write operation only occurs during the line sync. period, giving - maximum of 15625 operations per second. In the fast mode, the read/write operation is carried out within $800 n s$ for the 512 pixels horizontal resolution and $1.6 \mu s$ for the 256 pixels. The disadvantage of this mode is that during write operations the outputs of the memory go tristate which results in a white flash on the output, if writing occurs during the active I ine.

\section{A1. 4 Analogue Video Output}

The output impedance of the analogue video signal is $75 \Omega$ with a level of $1 \vee v$ peak-to-peak. This comprises of a $0.7 \vee v i d e o s i g n a l$ and $a 0.3 \vee$ syncs. signal.

\section{A1. 5 Registers}

Figure A1.3 shows the locations of the various registers used by the framestore and their memory mapped address on the $1 \mathrm{MHz}$ Bus.

The functions of these registers is as follows:

\section{(i) $\times \&$ Y Registers}

These are latches which hold the $X$ and $Y$ address of the particular pixel to be accessed by the microcomputer.

\section{(ii) Store Select}

This register indicates which store is being accessed at any time. When using the 256 by 256 and 512 by 256 pixel resolutions there are 4 and 2 stores respectively. 


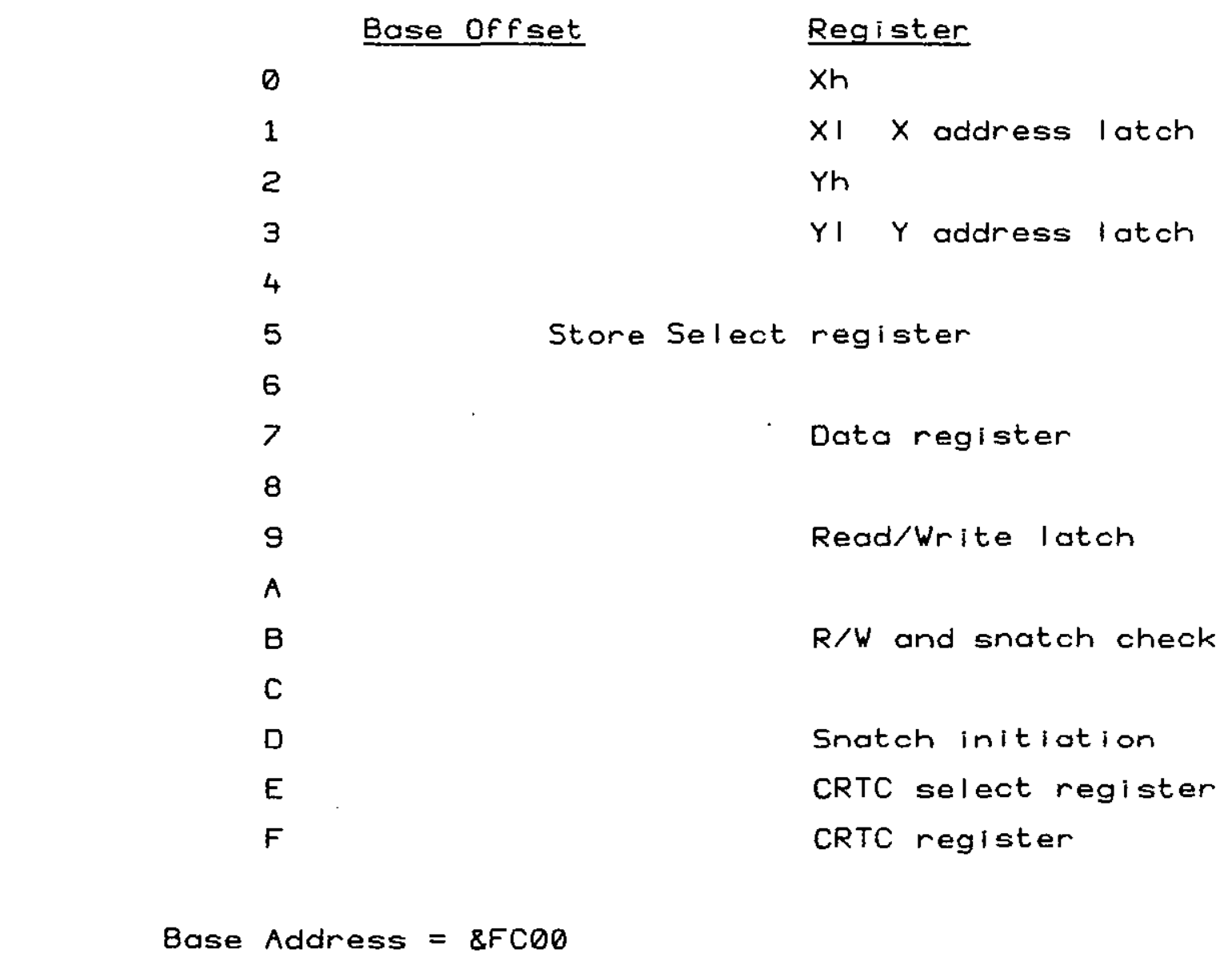

Fig. A1.3 Memory Map of Framestore Registers

256 by 256

FST. No. $\quad$ R4 R12 R13 REGISTERS R4 R12 R13

$\begin{array}{lllllll}1 & 00 & 00 & 00 & 00 & 00 & 00 \\ 2 & 01 & 08 & 00 & 01 & 10 & 00 \\ 3 & 02 & 10 & 00 & \mathrm{~N} / \mathrm{A} & \\ 4 & 03 & 18 & 00 & \mathrm{~N} / \mathrm{A} & \end{array}$

Fig. A1.4 Register Contents for Store Access and Display 
There are two forms of selection which must be specified;

(a) which store is selected for microcomputer access. (b) which store is to be displayed.

The first is achieved by using the store select register which is register four (R4) and the second by altering the contents of the CRTC registers (R12, R13) within the CRT controller. Fig. A1.4 shows the contents of these registers for the various framestore display and access configurations.

\section{(iii) Data}

The six least significant bits of the 8 bit register are used to determine the value of the data of the pixel pointed to by the $X$ and $Y$ registers.

\section{(IV) $R / W$ Latch}

This latch is used to indicate whether a read or write is required, depending on the values of the least significant bit.

LSB $=0 \quad \ldots \ldots$ write to store.

LSB $=1 \ldots$ read from store.

\section{(v) CRT Controller Initialisation}

The CRT controller contains a number of registers enabling the software programming of pixels per line. Iines per field. displayed store etc. . On start these registers have to be initialised. Figure A1.5 shows the contents of the registers required for the three possible display options. 
A1. 6 Power Requirements

The framestore requires the following power supplies:

$$
\begin{array}{r}
+5 \vee \ldots 1.5 \mathrm{~A} \\
+12 \vee \ldots 250 \mathrm{~mA} \\
-12 \vee \ldots 400 \mathrm{~mA}
\end{array}
$$

Register

256 by 256

$R 0 \quad 50$

R1 40

R2 42

R3 36

R4 27

R5 01

R6 20

R7 22

R8

R9 07

R10 00

R11

R12 $\quad 00$

R13 00

R14 00

R15 00

R16 00

R17 00

R18 00

R19 00
Resolution Option

512 by 256

512 by 512

$A O$

A1

80

80

88

84

3C

$3 \mathrm{C}$

27

40

01

00

20

40

22

44

01

03

07

06

00

00

00

$\emptyset 0$

00

00

00

00

00

00

00

00

00

00

00

00

00

00

00

00

Flg. A1.5 CRTC Register contents for Framestore Initialization 


\section{THE VELOCITY OF SOUND IN THE SEA}

\section{A2. 1 Introduction}

The transmission of acoustic signals in water is very dependant upon the velocity of propagation of the signal. The most generally accepted formula for the velocity of sound in water is by Wilson [42], but it contains a large number of high order terms.

A more practical formula is by Leroy [43] and is accurate to $0.1 \mathrm{~m} / \mathrm{s}$ for temperatures less than $20{ }^{\circ} \mathrm{C}$ and depths less than $8 \mathrm{~km}$. The formula for the velocity of sound in the sea, $c$, is

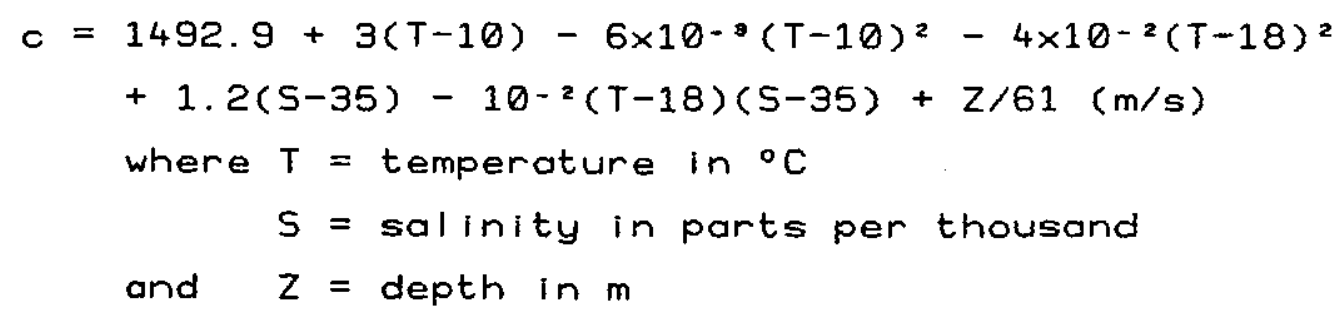

\section{A2. 2 Velocity Profiles}

A typical deep sea velocity profile is shown in Fig. A2.1. In the surface layer the velocity of sound is susceptible to changes in temperature due to heating. cooling and wind action. This layer may contain a mixed 


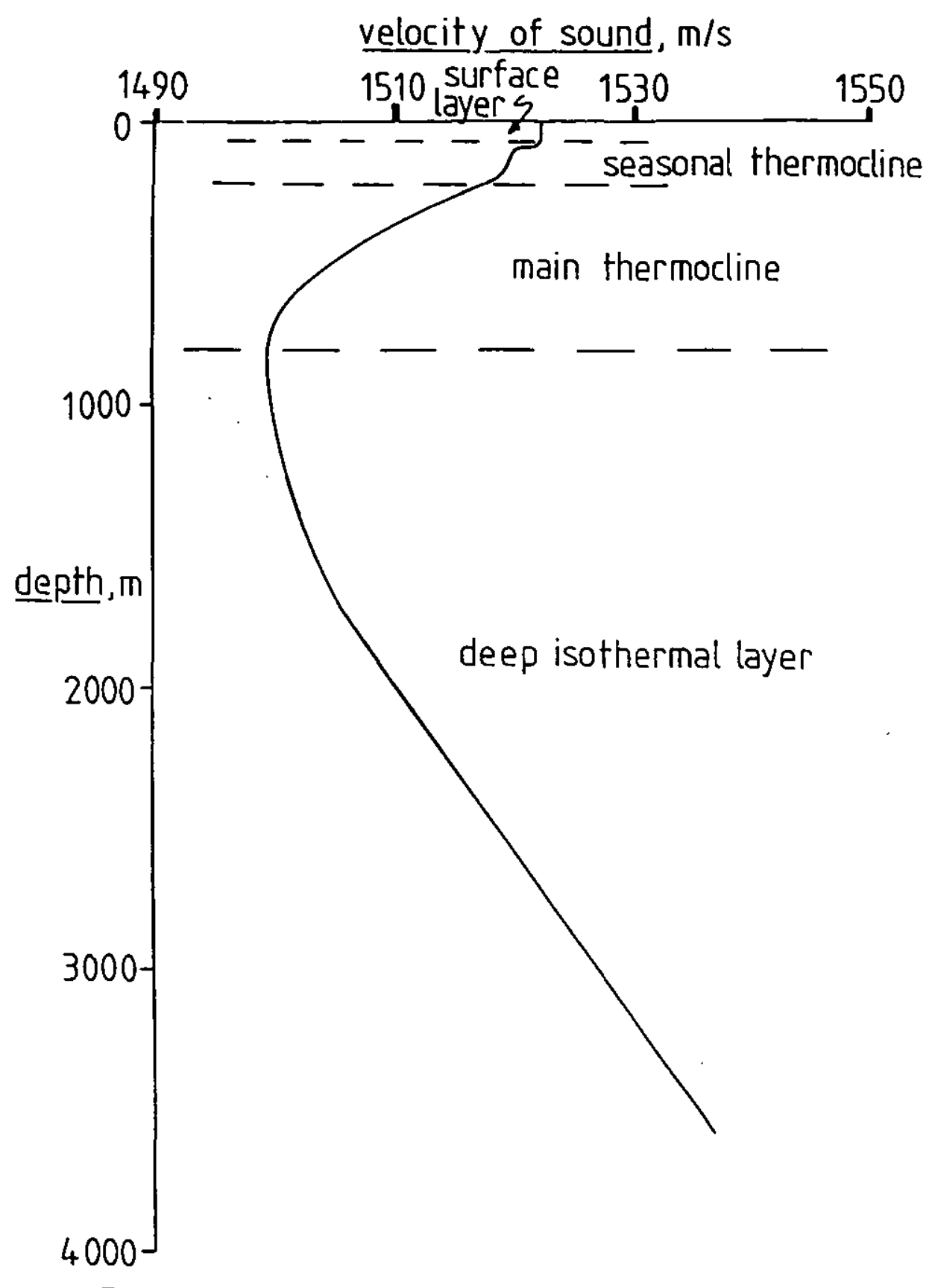

Fig. A2.1 Typical Deep-sea Velocity Profile. 
layer of isothermal water that forms channels for the sound to travel long distances. In sunny and calm conditions the temperature decreases with depth. The seasonal thermocline lies beneath the surface layer, here the temperature decreases with depth. During summer the seasonal thermocline is well defined and strong. in winter it is indistingulshable from the surface layer. The main thermocline lies beneath the seasonal thermoctine and is only offected slightly by seasonal changes. Below the main thermocline lies the deep isothermal layer. having a nearly constant temperature of $39^{\circ} \mathrm{F}$. in which the velocity of sound increases with depth because of the effect of increasing pressure (with depth) on sound velocity.

The thickness and occurence of these layers varies with the season, time of day. lattitude and the weather conditions. In shallow waters the velocity profile tends to be irregular and unpredictable and is greatly influenced by surface heating and cooling, salinity changes and water currents.

The velocity gradient influences the propagation of sound and ray theory can be used to estimate the paths of sound waves as they travel through the water.

\section{A2. 3 The Sea Surfoce and Bottom}

The surface of the sea and the sea bottom act as reflectors and scatterers of sound. They have a significant effect on the propogation of sound in most cases, where the source or recelver is near the surface or bottom, or when working in shallow waters.

When a negative velocity gradient exists just 
beneath the sea surface, a shadow is cast by the surface. in the sound field of a shallow source. Fig. A2.2 illustrates this effect for a sound source at a depth of $30 \mathrm{~m}$. The diagram shows the various paths taken by the sound as it travels through the water. when the tilt angle of the source is altered from zero degrees to nine degrees. With a constant sound velocity gradient. A shadow zone, is an area where the sound does not penetrate. due to the bending of path taken by the sound as it travels from a source. This bending is a function of the sound velocity gradient, and when there is a constant sound velocity, the sound travels in straight 1 ines.

Sound trovels large distances due to the effects of ducts in which the signal is prevented from spreading in all directions and remains confined within certain boundaries. These channels are formed due to the velocity profile and the surface and bottom of the sea. Fig A2.3 shows a duct formed near the sea surface due to a discontinuity in the sound velocity profile at a depth of $60 \mathrm{~m}$. 


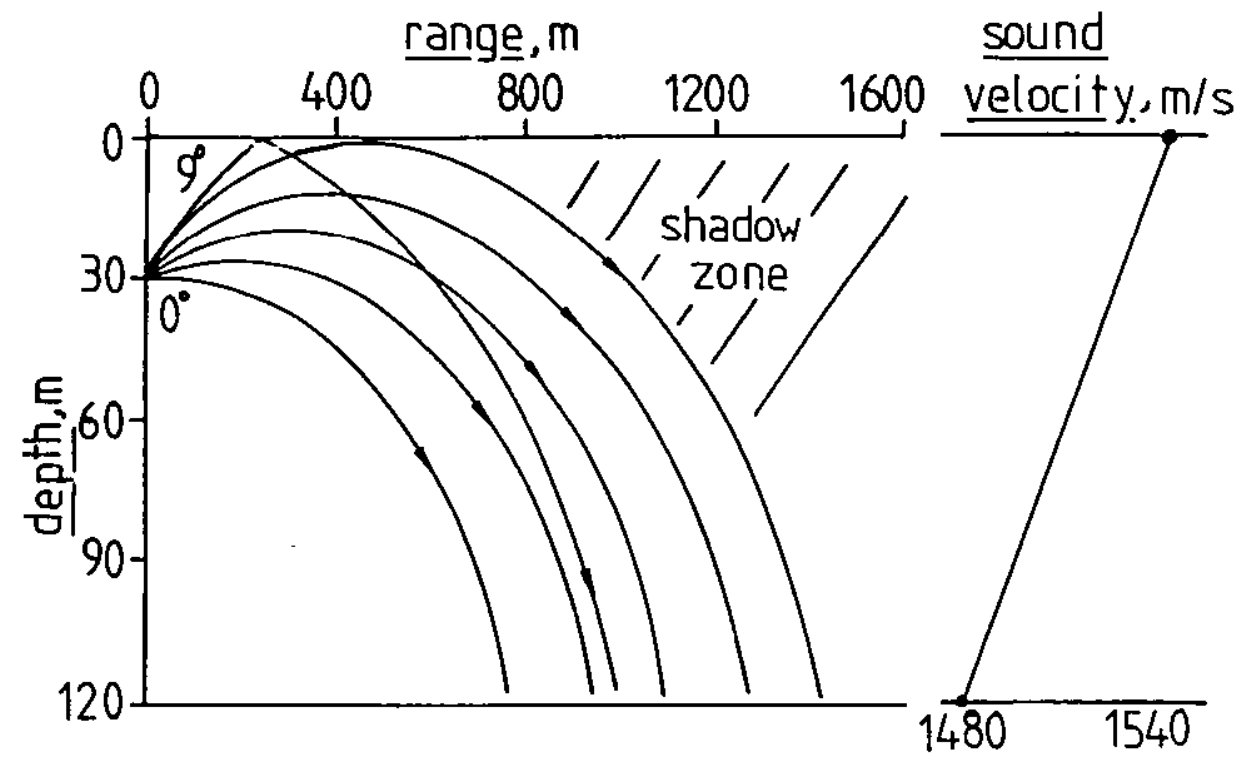

Fig.A2.2 Ray Diagram showing Shadow Zone.

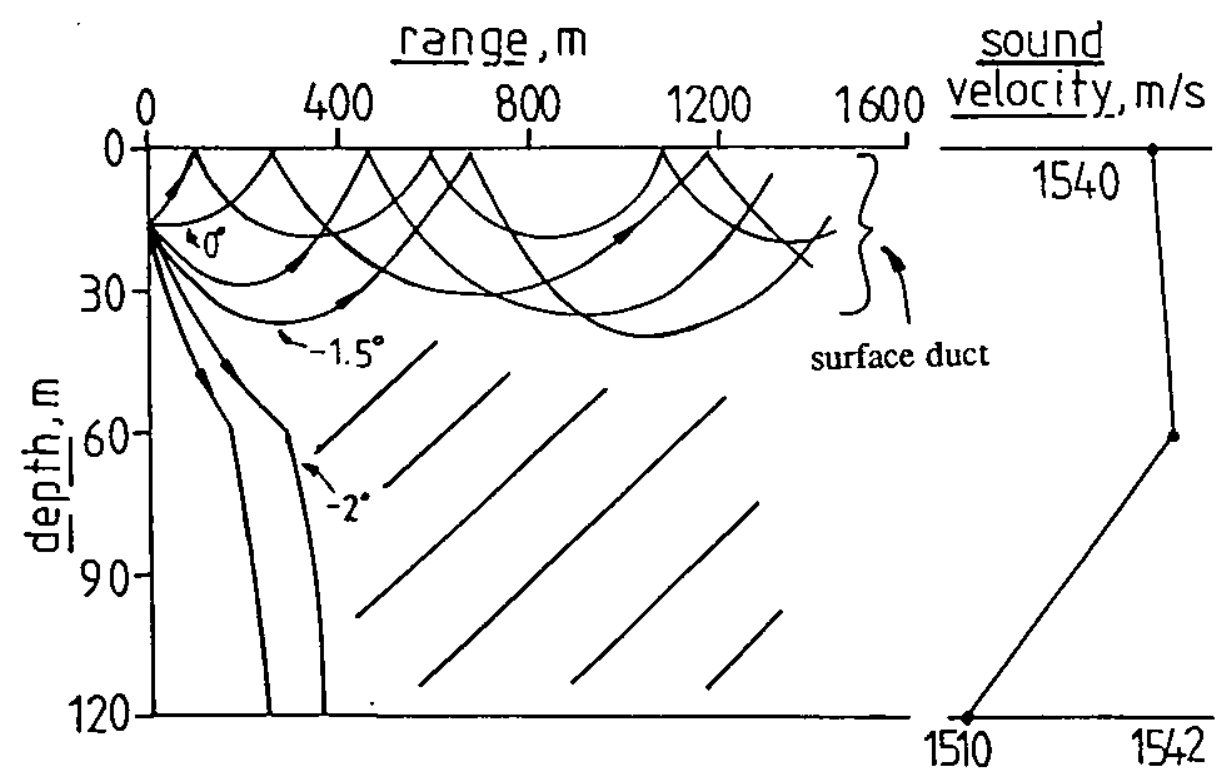

Fig. A2.3 Transmission Ducts in the Sea. 


\section{STEPPER MOTOR SPECIFICATIONS}

\section{A3. 1 Introduction}

The specifications and the pin connections of the stepper motors and the drive cards will be briefly discussed.

\section{A3. 2 Control Signals}

\section{A3.2.1 Mode Control}

When this signal is OV a 4 step sequence is generated i.e. 200 steps/rev motor will produce 200 steps/rev. When this signal is $0 / C$ a 8 step sequence is generated resulting in a 200 steps/rev. motor producing 400 steps/rev.

$$
\begin{aligned}
& \text { Moximum } 0 / C \text { Voltage }=+6 \mathrm{~V} \\
& \text { Maximum } 5 / C \text { current }=10 \mathrm{~mA}
\end{aligned}
$$

\section{A3.2.2 Direction}

This signal controls the stepping sequence and hence the direction of rotation of the stepper motor. It should only be altered when the stepper motor is stationary.

$$
\begin{aligned}
& \text { Maximum } 0 / C \text { Voltage }=+14 \mathrm{~V} \\
& \text { Maximum S/C Current }=40 \mathrm{~mA}
\end{aligned}
$$

If the 2 sections of the same coll (for example. A with 
$\bar{A}$ and $B$ with $\bar{B}$ are interchanged, the direction of rotation will reverse.

\section{A3.2.3 Step Pulse}

This input accepts an external pulse source to step the stepper motor. To use the local osclllator on the board this signal should be high impedance. Minimum pulse width $=50 \mu s$.

\section{A3. 3 Stepper Motor Specification}

Type Astrosyn - No. 34PMC004 - 4 phase. $0.7 \mathrm{~A}$ per phase, $14 \mathrm{~V}$ rated, $20 \Omega$ winding resistance (per phase)

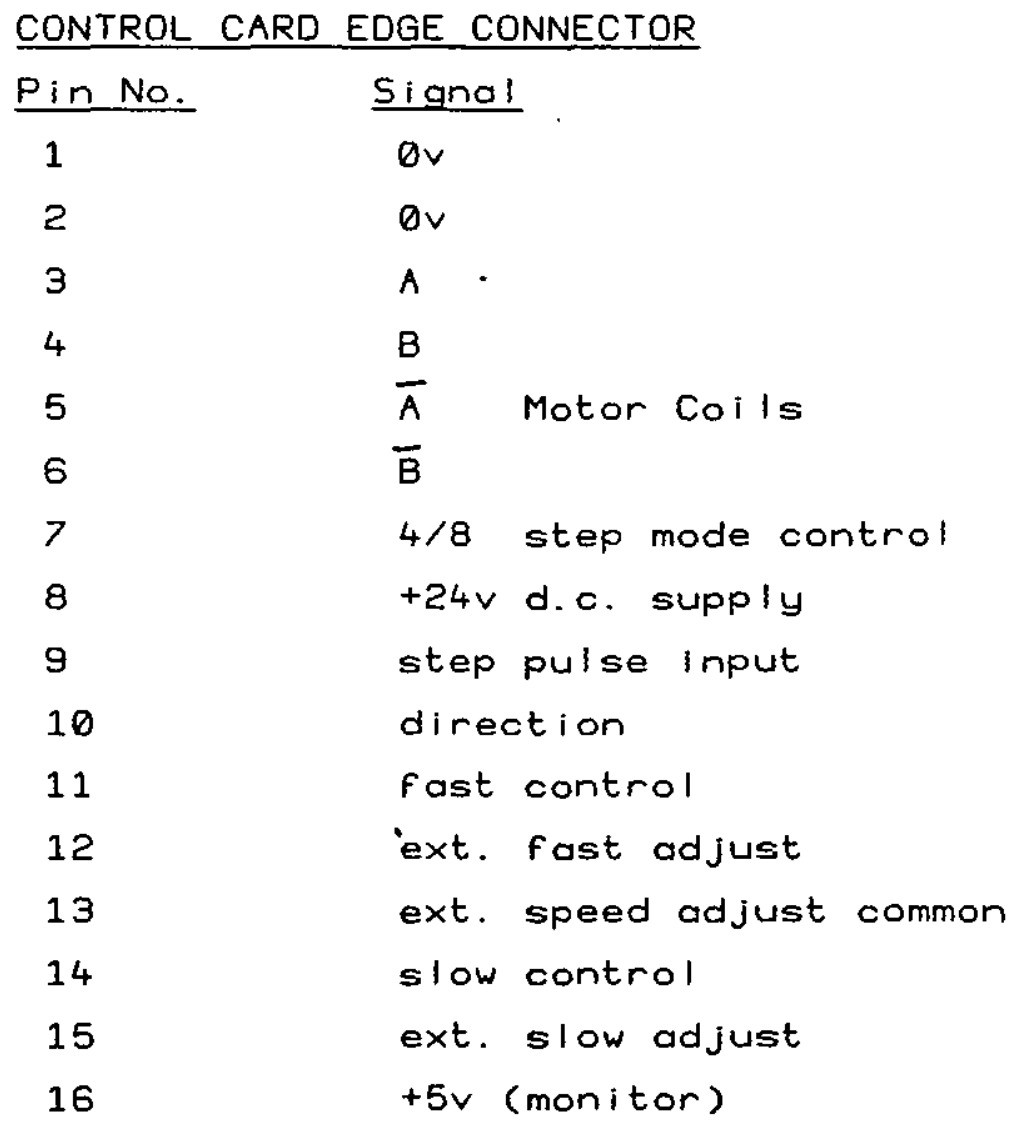

Fig. A3. 1 Drive Board Edge Connections 


\section{A3. 4 Power Supply}

Fig. A3.2 shows the circuit diagram of the power supply built to power the two stepper motors. The values of the components in the intermediate circuit, shown in Fig. A3. 3. were calculated from:

$$
\begin{array}{ll}
R 1=R / 2, & R 2=R \\
R=\frac{24-V m}{2 I p h} & V m=\text { rated motor coil voltage }
\end{array}
$$$$
\text { Iph }=\text { rated current per phase }
$$$$
\text { when } 2 \text { phases are energized. }
$$

Wattage of $R 1$ and $R 2=(24-V m)$ Iph Watts

It is recommended that 2 times the wattage calculated values be used to avoid high surface temperatures. The following values of resistance were calculated:

$$
R=\frac{24-14}{2 \times 0.7}=7.1 \Omega
$$

$$
\text { therefore } \begin{aligned}
\mathrm{R} 1 & =3.55 \Omega \\
\mathrm{R} 2 & =7.1 \Omega
\end{aligned}
$$

Required power rating $=(24-14) * 0.7=7$ watts

The nearest value of resistance available were used;

$$
\begin{aligned}
& R 1=3.3 \Omega .20 \mathrm{~W} . \\
& R 2=6.8 \Omega, 20 \mathrm{~W} .
\end{aligned}
$$




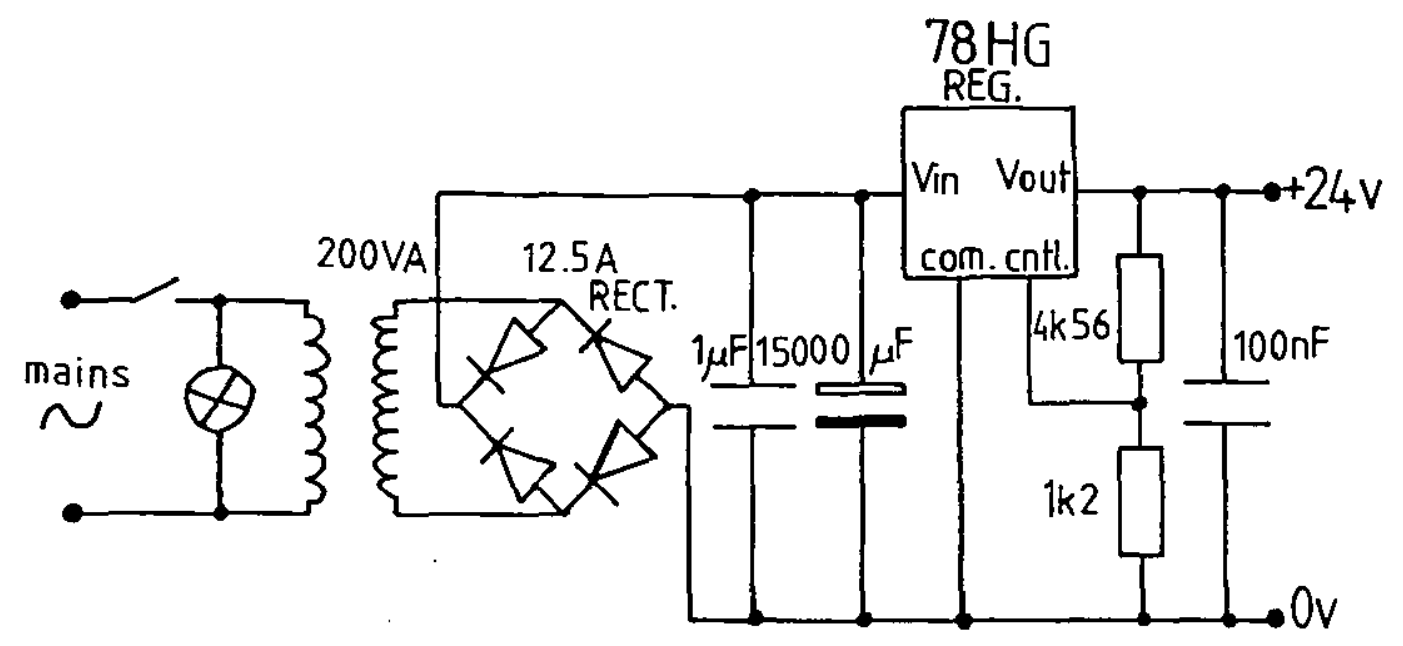

Fig. A3.2 Stepper Motor Power Supply.

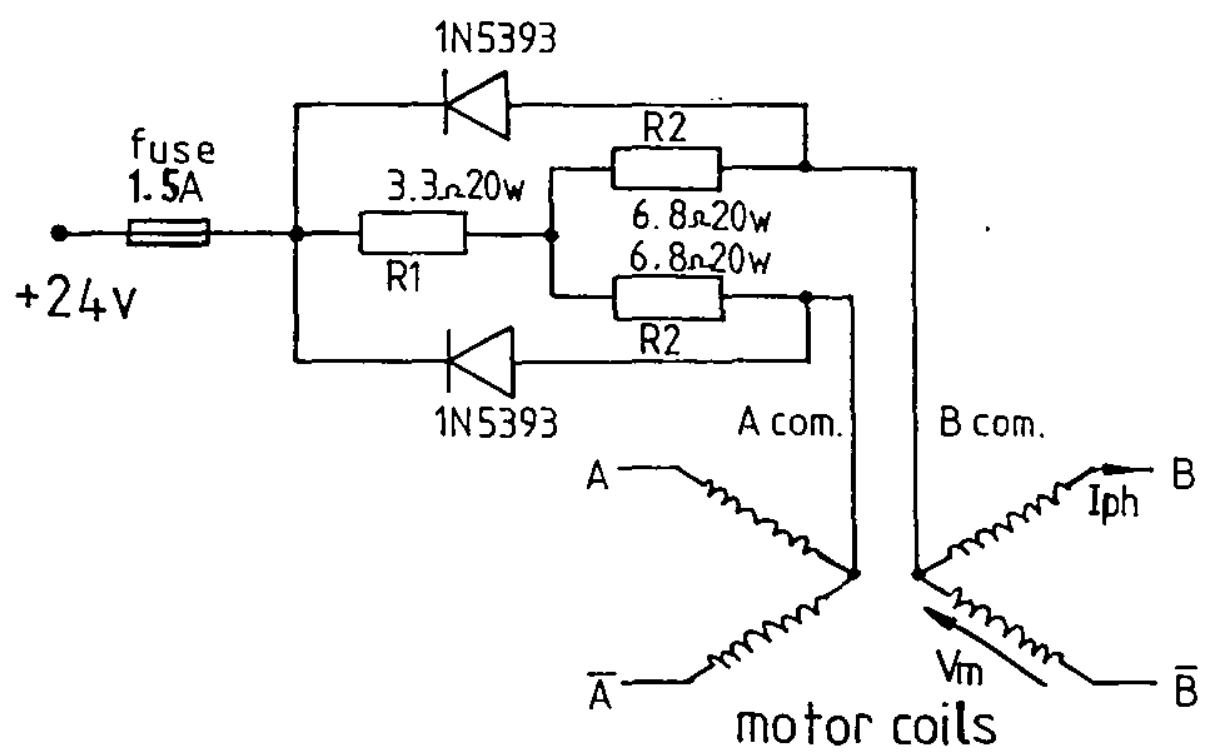

Fig. A3.3 14v Supply for the Stepper Motor Coils. 


\section{LISER PORT CONNECTIONS}

Pin No. Signal Pin No. Signal

\begin{tabular}{|c|c|c|c|c|}
\hline 1 & $+5 v$ & 2 & $\mathrm{cb}_{1}$ & $n / c$ \\
\hline 3 & $+5 v$ & 4 & $\mathrm{cb} 2_{-}$ & $n / c$ \\
\hline 5 & $\theta_{v}$ & 6 & $P b O_{-}$ & END detect \\
\hline 7 & $\theta v$ & 8 & $\mathrm{~Pb}_{1}$ & $n / c$ \\
\hline 9 & $\theta v$ & 10 & $\mathrm{~Pb}_{2}$ & $n / c$ \\
\hline 11 & $\theta_{v}$ & 12 & Pb3_ & SELect \\
\hline 13 & $\theta v$ & 14 & $\mathrm{~Pb}_{4}$ & START PUIse \\
\hline 15 & Ov & 16 & $P b 5$ & DIRection \\
\hline 17 & $\theta v$ & 18 & Pb6_ & MODE \\
\hline 19 & Or & 20 & $\mathrm{~Pb} 7_{-}$ & STEP pulse \\
\hline
\end{tabular}




$$
\text { APPENDIX FIVE }
$$

THE REQEIVER BOARD TO

MEMORY BOARD RIBBON CABLE [RBI]

Pin No. SIGNAL

\begin{tabular}{|c|c|c|c|}
\hline 1 & D11 & 2 & NWE \\
\hline 3 & 010 & 4 & $c 2$ \\
\hline 5 & DQ & 6 & $\theta v$ \\
\hline 7 & $\mathrm{D} 8$ & 8 & $\theta_{V}$ \\
\hline 9 & Q7 & 10 & $\theta_{V}$ \\
\hline 11 & D6 & 12 & $\theta_{v}$ \\
\hline 13 & D5 & 10 & $\theta_{v}$ \\
\hline 15 & 04 & 16 & $\theta_{v}$ \\
\hline 17 & 03 & 18 & $\partial_{v}$ \\
\hline 19 & $\mathrm{D} 2$ & 20 & $\theta_{v}$ \\
\hline 21 & 01 & 20 & $\theta_{v}$ \\
\hline 23 & 00 & 24 & $c 3$ \\
\hline 25 & START pulse & 26 & $n / c$ \\
\hline
\end{tabular}




\section{APPENDIX SIX}

EULL CIRCUIT DLAGRAM OF THE TRANSMIT PULSE AND REFERENCE GENERATOR BOARD 


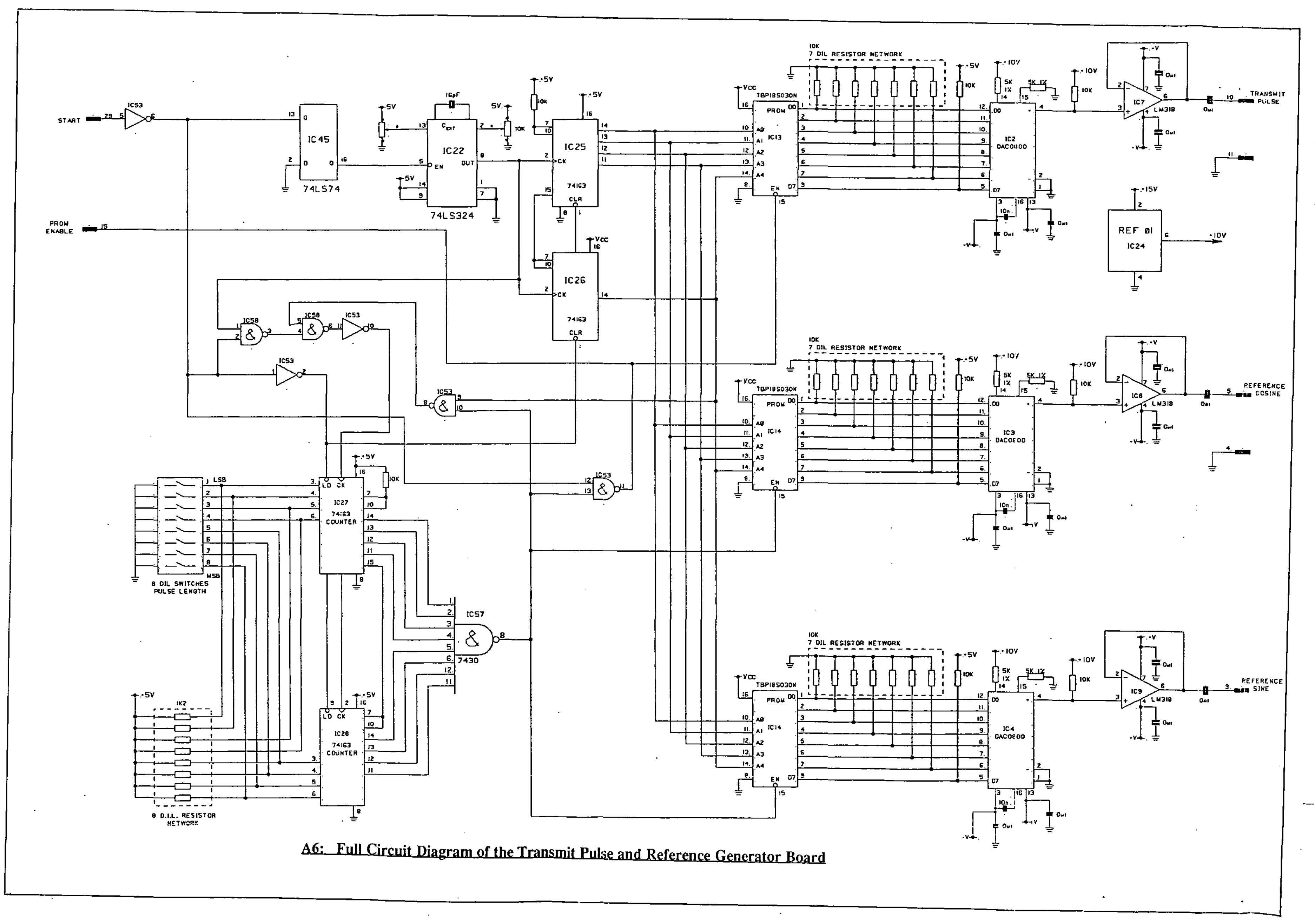




\section{APPENDIX SEVEN}

FULL CIRCUIT DIAGRAM OF THE MEMORY BOARD 


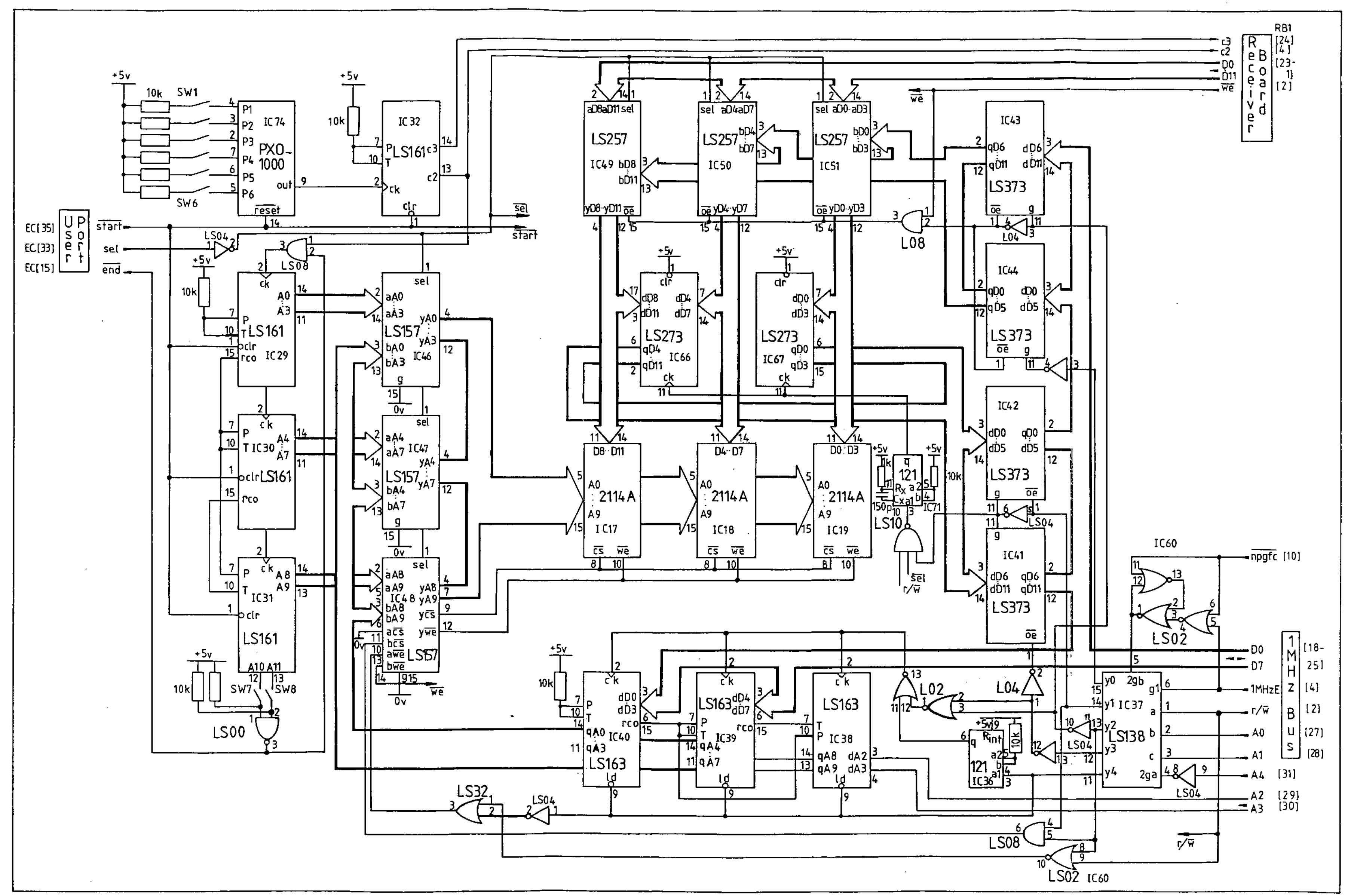

A7: Full Circuit Diagram of the Memory Buffer Board 
APPENDIX EIGHT

FULL CURCUIT DIAGRAM OF THE BASEBAND RECEIVER BOARD 


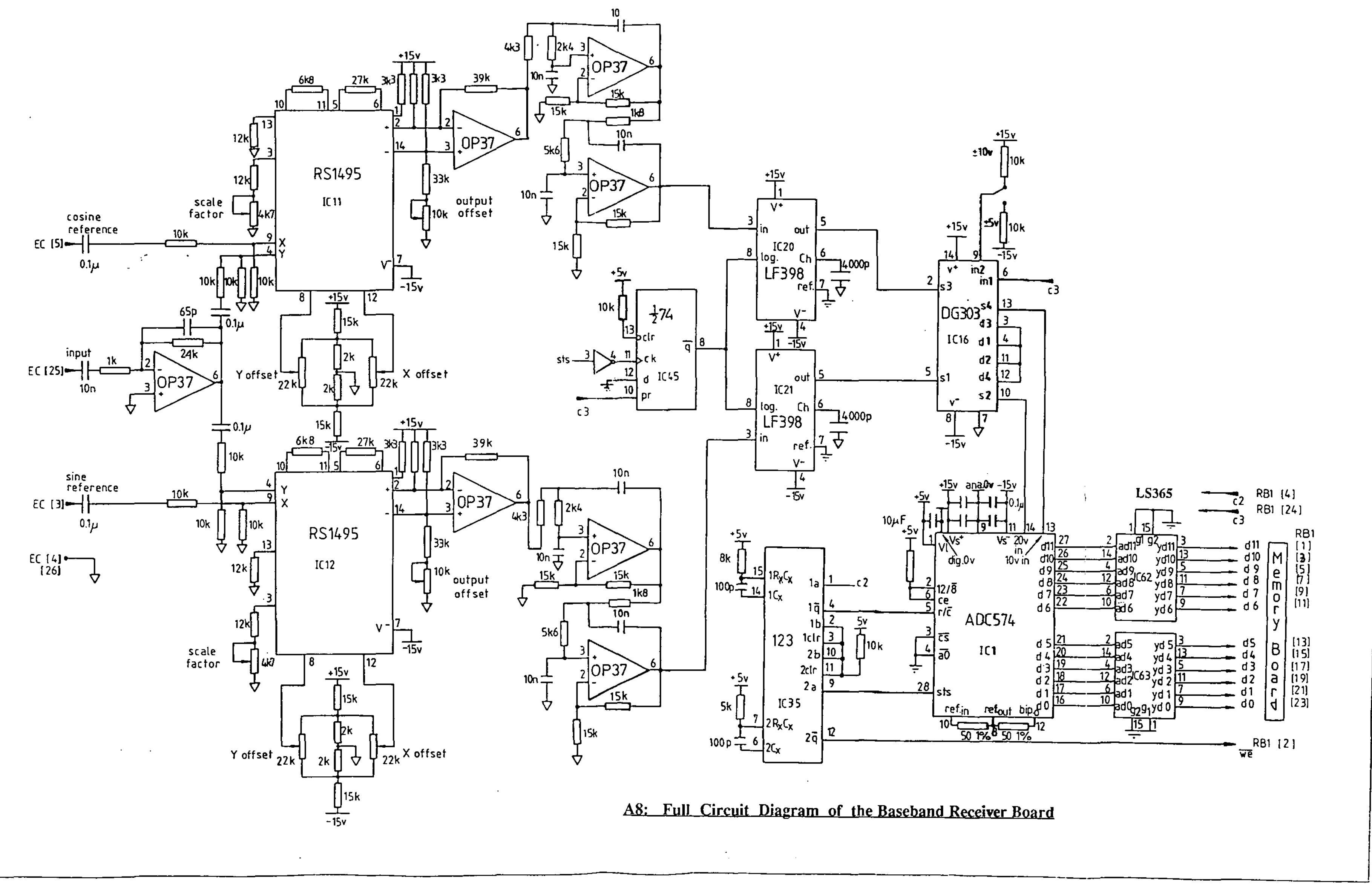


